Universidad de Salamanca

Instituto de Neurociencias de Castilla y León (INCyL)

Auditory Neurophysiology Laboratory

\author{
MECANISMOS FUNCIONALES DE LA \\ ADAPTACIÓN ESPECÍFICA A ESTÍMULOS Y LA \\ DETECCIÓN DE LA NOVEDAD EN EL CEREBRO AUDITIVO
}

FUNCTIONAL MECHANISMS OF STIMULUS-SPECIFIC ADAPTATION AND

DEVIANCE DETECTION IN THE AUDITORY PATHWAY

Thesis submitted by

DANIEL DUQUE DONCOS

\begin{abstract}
To obtain the
Degree of Doctor in Neuroscience by the University of Salamanca

And the International Doctor Mention
\end{abstract}

Supervised by

Dr. Manuel Sánchez Malmierca

Salamanca, September 2014 



\section{ORIGINAL PUBLICATIONS}

This thesis is a compendium of the following publications:

Peer reviewer published articles:

- Topographic distribution, frequency, and intensity dependence of stimulus-specific adaptation in the inferior colliculus of the rat (2012) Daniel Duque ${ }^{1}$, David Pérez-González ${ }^{1}$, Yaneri A. Ayala ${ }^{1}$, Alan R. Palmer ${ }^{3}$ and Manuel S. Malmierca ${ }^{1,2}$ ${ }^{1}$ Auditory Neurophysiology Unit, Laboratory for the Neurobiology of Hearing, Institute of Neuroscience of Castilla y León, 37007 University of Salamanca, Salamanca, Spain

2 Department of Cell Biology and Pathology, Faculty of Medicine, University of Salamanca, 37007 Salamanca, Spain

${ }^{3}$ Institute of Hearing Research, Medical Research Council, Nottingham NG7 2RD, United Kingdom Journal of Neuroscience 32:17762-17774. doi: 10.1523/JNEUROSCI.3190-12.2012.

- Modulation of stimulus-specific adaptation by GABAA receptor activation or blockade in the medial geniculate body of the anaesthetized rat (2014)

Daniel Duque ${ }^{1,2}$, Manuel S. Malmierca ${ }^{1,3}$ and Donald M. Caspary ${ }^{2}$ ${ }^{1}$ Auditory Neurophysiology Unit, Laboratory for the Neurobiology of Hearing, Institute of Neuroscience of Castilla y León, 37007 University of Salamanca, Salamanca, Spain

${ }^{2}$ Department of Pharmacology, Southern Illinois University School of Medicine, Springfield, IL, USA

${ }^{3}$ Department of Cell Biology and Pathology, Faculty of Medicine, University of Salamanca, 37007 Salamanca, Spain Journal of Physiology 592:729-743. doi: 10.1113/jphysiol.2013.261941. 
- Stimulus-specific adaptation in the inferior colliculus of the mouse: anesthesia and spontaneous activity effects. Daniel Duque ${ }^{1}$ and Manuel S. Malmierca ${ }^{1,2}$ (2014) ${ }^{1}$ Auditory Neurophysiology Unit, Laboratory for the Neurobiology of Hearing, Institute of Neuroscience of Castilla y León, 37007 University of Salamanca, Salamanca, Spain ${ }^{2}$ Department of Cell Biology and Pathology, Faculty of Medicine, University of Salamanca, 37007 Salamanca, Spain Brain Structure \& Function (Epub ahead of print) doi: 10.1007/s00429-014-0862-1.

\section{Article under review:}

- The width of the frequency channels determines stimulus-specific adaptation in the inferior colliculus of the rat. Xin Wang ${ }^{1^{*}}$, Daniel Duque ${ }^{1^{*}}$ and Manuel S. Malmierca ${ }^{1,2}$ (2014) ${ }^{1}$ Auditory Neurophysiology Unit, Laboratory for the Neurobiology of Hearing, Institute of Neuroscience of Castilla y León, 37007 University of Salamanca, Salamanca, Spain ${ }^{2}$ Department of Cell Biology and Pathology, Faculty of Medicine, University of Salamanca, 37007 Salamanca, Spain

* these authors contributed equally to this work Under review 
MANUEL SÁNCHEZ MALMIERCA, PROFESOR TITULAR DEL DEPARTAMENTO DE

BIOLOGÍA CELULAR Y PATOLOGÍA DE LA UNIVERSIDAD DE SALAMANCA E

INVESTIGADOR DEL INSTITUTO DE NEUROCIENCIAS DE CASTILLA Y LEÓN

\section{CERTIFICA}

Que la tesis doctoral titulada:

\section{FUNCTIONAL MECHANISMS OF STIMULUS-SPECIFIC ADAPTATION AND DEVIANCE DETECTION IN THE AUDITORY PATHWAY}

Se realiza bajo el formato de compendio de artículos, ha sido redactada en inglés, contiene un resumen en español y describe el trabajo de investigación realizado por Don Daniel Duque Doncos bajo mi dirección durante los últimos 4 años.

La memoria de este estudio recoge un análisis detallado y exhaustivo de los mecanismos de adaptación específicos a estímulos en el colículo inferior y el cuerpo geniculado medial del tálamo auditivo. Los datos presentados en esta memoria constituyen una aportación original y puedo afirmar que ponen de manifiesto un gran avance y progreso en el área de las Neurociencias.

Por todo ello, considero que esta tesis reúne la calidad y rigor científicos necesarios para que sea defendida en la Universidad de Salamanca como requisito para que Don Daniel Duque Doncos opte al grado de 'Doctor' con 'Mención Internacional' por la Universidad de Salamanca.

Y para que así conste, firmo el presente certificado,

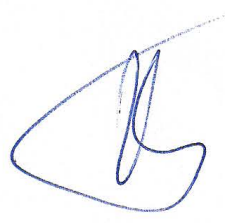

Prof. Manuel Sánchez Malmierca Salamanca, 25 de Septiembre de 2014 

No es el pensador y el artista el que recibe diplomas y subvenciones, lo es el que sería feliz sin pensar ni expresar lo que lleva dentro, pero que no puede evadirse de hacerlo, porque le obligan a ello dos fuerzas invisibles: su necesidad interior y su amor a los hombres. No hay artistas inflados, gozadores y satisfechos de sí.

León Tolstói

¿Qué debemos hacer? (extraído del prólogo de Ana Karénina) 
This work has been supported by a fellowship from the Spanish Ministry of Education and Science (MEC; BES-2010-035649), and different grants from the Spanish Ministry and Science and Innovation (MICINN, BFU2009-07286), the Junta de Castilla y León (JCYL-UE, GR221), the European Union in the frame of the ERA-NET NEURON (EUI2009-04083), the National Institutes of Health (DC000151) and the Spanish Ministry of Economy and Competitiveness (MINECO, BFU2013-43608-P). 


\section{ACKNOWLEDGMENTS}

Me gustaría dar las gracias, por encima de todo, a mi supervisor y tutor Dr. Manuel S. Malmierca. Primero, por brindarme la oportunidad de trabajar en lo que más me gusta, la Neurociencia. Segundo, por su apoyo y paciencia, por saber estar a mi lado cuando los experimentos no van bien y siempre estar dispuesto a compartir su experiencia con una metáfora. Por su fantástica gestión de equipo y las terapias psicológicas gratuitas. Gracias.

A todos los miembros del laboratorio de Audición, especialmente a David por estar conmigo al principio de mi tesis y enseñarme las bases de la buena ciencia y a Nacho por sus fantásticos consejos metodológicos: ir con dudas a preguntarle algo siempre me dio mucho más de lo que requería. A Javier y Xin, por su buena compañía y sobresaliente ciencia, por hacer del lugar de trabajo algo más que eso.

I am most grateful to Dr. Don Caspary for receiving me so nicely in his laboratory, during my stay in Springfield. I am also thankful to all the people who contributed with magnificent comments on my work over these years, especially to Dr. Alan Palmer, Dr. Israel Nelken and Dr. Carles Escera.

A todos mis compañeros del INCYL, especialmente a la comunidad del comedor. A Flora, Yan, Vero, Bego y Marta que más que compañeras son amigas. A Mercedes por los dos primeros años en el INCyL. A Irene por estos dos últimos.

A tot el grup de Manyanet Community, que han fet que la distància entre Salamanca i Barcelona sigui molt petita encara que ens veiéssim poc... $i$ massa gran quan us trobava a faltar, que era sovint.

A mi padre por ser un apoyo constante durante estos cuatro años. A mi madre y a mi hermana por estar ahí pese a la distancia. Gràcies!

Y, por supuesto, a Cris. Por hacer que todo sea más fácil. 

TABLE OF CONTENTS

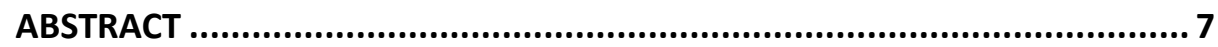

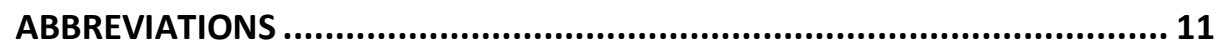

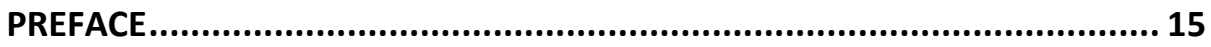

Neural coding of the natural environment................................... 17

Efficient coding hypothesis...................................................... 18

Sensory adaptation ............................................................. 20

INTRODUCTION ..................................................................... 23

The auditory system ........................................................... 25

The inferior colliculus (IC) ...................................................... 29

The medial geniculate body (MGB) ........................................ 32

The lemniscal and non-lemniscal pathway................................. 33

Auditory scene analysis and deviance detection .......................... 35

Mismatch negativity $(\mathrm{MMN})$..................................................... 36

Stimulus-specific adaptation (SSA) ....................................... 38

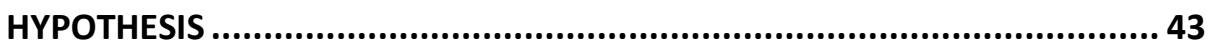

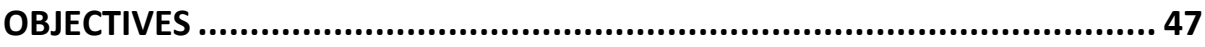

RESULTS ................................................................................. 51

Study I: Frequency and level dependence of SSA .......................... 53

Study II: GABAergic modulation of SSA .................................... 71

Study III: SSA in the awake and anesthetized IC ............................ 89

Study IV: Frequency channels determines SSA ........................... 103

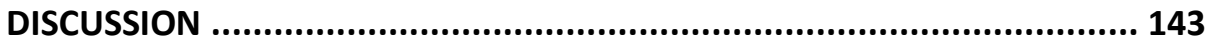

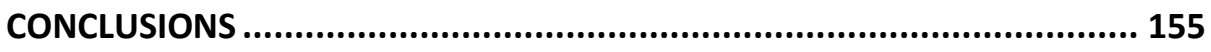

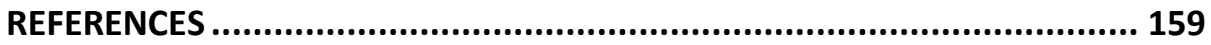

ANNEX: SPANISH SUMMARY................................................. 181 

ABSTRACT 

The objective of this Doctoral Thesis was to shed light on the functional mechanisms of stimulus-specific adaptation (SSA), a likely neuronal basis for deviance detection in the auditory system. Four electrophysiological studies have been performed by extracellularly recording isolated neurons during auditory stimulation in subcortical nuclei of the anesthetized rat and awake mouse: the inferior colliculus and the medial geniculate body of the thalamus. All the experiments has been performed using the oddball paradigm, which evaluates the differential responsiveness to frequent (standards) and rare sounds (deviants).

Study $\mathrm{I}$ is a detailed analysis of the neuronal features that are affecting SSA sensitivity in the inferior colliculus of the anesthetized rat. I show that SSA is frequency and intensity dependent, it is better represented in the earliest portions of the response and that the width of the frequency response area of the neuron is related to SSA sensitivity.

Study II used the microiontophoretic technique to show that GABAergic inhibition is not generating SSA in rat. I show that Inhibition modulates SSA in a gain control manner. This information is reinforced in Study III, where I implanted a novel technique in the laboratory to record in awake mice. Recordings in unanesthetized animals demonstrate that SSA is a genuine phenomenon that seems more active in sleep-like states.

Last, study IV shows that SSA is sensitive to frequency- but not to intensity deviants. It further demonstrates that SSA is dependent on the frequency inputs that generate the frequency response area of the neurons.

Studies I and IV suggest that SSA might be related to an excitatory input segregation, while studies II and III show that SSA is modulated by inhibition but not generated by an excitatory-inhibitory balance. Therefore, we hypothesized that input-specific synaptic depression could be generating SSA in subcortical nuclei. 

ABBREVIATIONS 

AC

CNIC

CSI

DNIC

FRA

IC

LNIC

MGB

MGD

MGM

MGV

MMN

NIA

PSTH

SI

SR

SSA

TRN
Auditory cortex

Central nucleus of the inferior colliculus

Common stimulus-specific index

Dorsal nucleus of the inferior colliculus

Frequency response area

Inferior colliculus

Lateral nucleus of the inferior colliculus

Medial geniculate body

Dorsal division of the medial geniculate body

Medial division of the medial geniculate body

Ventral division of the medial geniculate body

Mismatch negativity

Normalized index of adaptation

Peri-stimulus time histogram

Frequency specific index for stimulus-specific adaptation

Spontaneous rate

Stimulus specific adaptation

Thalamic reticular nucleus 

PREFACE 

One of the main epistemological questions has always been whether or not we are able to perceive the True image of the world ('reality as it is in itself'). Since the ancient Greek culture, Western philosophy has been divided, grosso modo, between the 'realism' from Aristotle and the 'epistemological dualism' from Plato. The famous Plato's Allegory of the Cave set the grounds of the Theory of Forms, which defend that ideas -and not the world we perceive through sensation- possess the highest and most fundamental kind of reality. Following that perspective, Immanuel Kant develop his masterpiece The Critique of Pure Reason (1781) which is the basis for 'transcendental idealism', later improved by Arthur Schopenhauer in his book The World as Will and Representation (1818) and perfectly illustrated in the opening sentence of the manuscript: 'The world is my representation'.

Many other perspectives have been developed since, but the main core remains, i.e., reality is not straightforwardly perceivable by our sensory systems. Interestingly, it does not seem necessary for the organisms to see the 'real' world, as only an adequate representation of nearby environment is required to provide an optimal response to survive.

\section{Neural coding of the natural environment}

During the last decades, a great deal of electrophysiological recordings carried out at different levels of the sensory systems have reinforced the idea that the brain is not trustworthy reproducing the world but simply reconstructing and reinterpreting it in order to provide a reliable response to the continuous flow of external inputs. This reliable response has only the purpose of survival. For this reason, the brain needs to create coherent and meaningful abstract representations from the complexity of the ever-changing world with a minimal amount of storage (Miller et al., 2003). Considering that much of the information received by an organism is 
highly redundant (Attneave, 1954), the information theory developed by Claude E. Shannon (1948) is critical in the signal processing that take place in the neurons. Information theory was initially designed for optimizing the transmission of messages over a noisy channel at the beginning of the $20^{\text {th }}$ century. The simple lineal model proposed by Shannon implies an information source that produces a message through a transmitter. The transmitter produces a signal suitable for transmission over a channel, which may be interfered by some noise. The receiver decodes the signal and reconstructs the message, finally received by the destination.

Shannon's information theory uses the concept of entropy to measure the uncertainty about a source of information, i.e., entropy is the average amount of information contained in a message. That is one of the fundamental concepts of information theory: the amount of information in a message has a defined and measurable value (bits). If a message has low probabilities to be received it has high levels of entropy. If we are sure a message is going to be received, the amount of information is zero. In summary, the less likely an event is, the more information it provides when it occurs.

\section{Efficient coding hypothesis}

A few years after Shannon's seminal work (1948), two different papers adapted information theory ideas to the biological field of sensory coding in the brain (Attneave, 1954; Barlow, 1961). Within the framework of information theory, Barlow proposed a link between environmental statistics and neural responses through the concept of coding efficiency. Barlow's model was based on three essential concepts:

- $\quad$ Password hypothesis: Sensory relays detect 'passwords' with a vital significance for the animal. In Barlow's words (1961): "the primary effect of the sensory messages an animal receives is not 
to enrich its subjective experience of the world but to modify its behavior in such a way that it and its species have a greater chance of survival. [...] The subjective sensations they would produce in ourselves may or may not be relevant".

- Controlled pass-characteristic hypothesis: Each nucleus in the sensory pathway act as a modifiable pass-filter. Sensitivity to one type of stimulus may be enhanced or attenuated depending on contextual circumstances. The same message in different surroundings may imply different responses as the 'password' significance may be altered.

- Redundancy-reducing hypothesis: To rebuild the message from the signals, the system needs to control the redundancy through adaptation. Again, in Barlow's words (1961): "laws of nature serve the purpose of bringing order and simplicity to our complex sensory experiences [...]; this seems to be the same idea as recoding to reduce the redundancy of our internal representation of the outer world".

Considering Shannon's model, a biological analogy could be as follows: a predator produces a sound and the prey's sensory pathway acts as a communication channel to transmit such signal. The spikes produced by the neurons through the sensory pathway efficiently code the signals, ultimately processed at high order brain regions that reconstruct the signal into a message. Environmental sound could be masking the message produced by the predator, but the system is able to extract the signal from the external noise (Figure 1). 


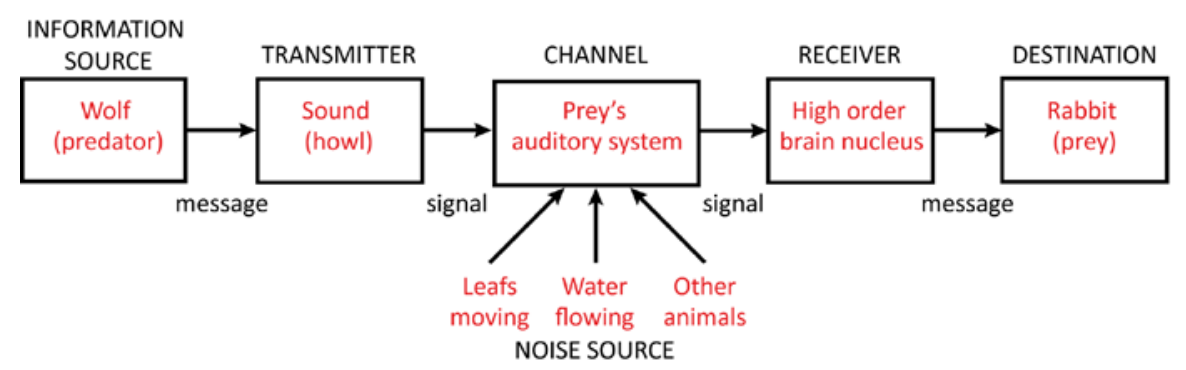

Figure 1. Information theory model adapted to the efficient coding hypothesis. Adapted and reinterpreted from Barlow, 1961.

Barlow's efficient coding hypothesis suggest that, given a finite capacity to transmit information, neural systems employ an optimally efficient coding strategy to represent the inputs that they typically process (Wark et al, 2007). In order to optimize efficiency the sensory system should adjust its outputs to match the relevant inputs that code most of the 'passwords' with key relevance for the animal survival. The optimal coding strategy depends on the statistics of the stimulus that the system represents (Wark et al., 2007) and the possibility to plastically adjust to them.

\section{Sensory adaptation}

Redundancy-reducing hypothesis assumes that sensory processing in the brain implies some levels of neuronal adaptation. If a neural system can change its coding strategy as the distribution of stimuli changes, adaptation would help neural systems to efficiently encode natural stimuli (Wark et al., 2007). The rational should imply integrative neuronal interactions in order to enhance determined responses depending on the environmental context. According to such idea, a recent paper theorized that the typical heterogenic response found in the same cortical region to the same stimulus is beneficial for sensory coding when the stimulus is decoded from the population response (Chelaru and Dragoi, 2008). 
During the last decades, several studies provided unquestionable evidences that all the sensory systems are able to efficiently code stimulus variations. A pioneer study in the insect compound eye demonstrated that the response of interneurons code for contrast variations (Laighlin, 1981). Years after, some visual researchers demonstrate adaptive rescaling to adjust to spatial correlations and image contrast (Smirnakis et al., 1997) and to the velocity of the stimulus (Brenner et al., 2000; Fairhall et al., 2001). All these adaptive processes begin to occur in the primary sensory receptor neurons but can also be observed in higher brain regions, like the cortex.

Recent experiments demonstrated the presence of adaptive processes that allows for efficient coding adjustments in the auditory(Lewicki, 2002; Ulanovsky et al., 2003; Dean et al., 2005; Dahmen et al., 2010), the somatosensory- (Katz et al., 2006; Maravall et al., 2007) and the olfactory system (Assisi et al., 2007; Kostal et al., 2008). All this experiments reinforce the idea that adaptation, as a passive process, is old-fashioned. Thus, adaptation is a dynamic phenomenon and allows animals to have an optimal representation of the world. 



\section{INTRODUCTION}



Survival depends critically on the ability to perceive relevant signals and separate from those unimportant. And neuronal adaptation seems to be closely involved in allowing such detection. Detecting strange odors in the middle of the night or visualizing a predator in the jungle are essential for survival, but the ability to detect novel sounds in the environment is fundamental to stay in an ever-changing world. Rare sounds indicate events of behavioral importance to which an individual must attend to survive.

\section{The auditory system}

The auditory system is specialized in processing environmental acoustic information. The biological importance of the auditory system of vertebrates lies in its ability to decode the temporal and spectral cues of sounds and provide animals with information about what the sounds mean and locate their origin. In many animals, sounds also serve as a basis for communication and in humans in particular is linked to language.

The extraction of useful information starts in the cochlea, where the sound is transduced to electrical signals. Previously, sound waves reach the tympanic membrane, where the mechanical energy is amplified and transmitted though the ossicles to the basilar membrane, in the cochlea. Due to the physical characteristics of the membrane, the pressure waves vibrate at different regions of the membrane, which defines the frequency of stimulation $(\mathrm{Hz}$, hertzs). The sensory hair cells, placed over the basilar membrane of the cochlea, are excited by the movement of the membrane and transduce the vibrations to electrical signals. Thus, tonotopy emerges in the cochlea (Figure 2), as individual hair cells are excited by different frequency ranges depending on the specific region of the basilar membrane (von Bèkèsy, 1960). 


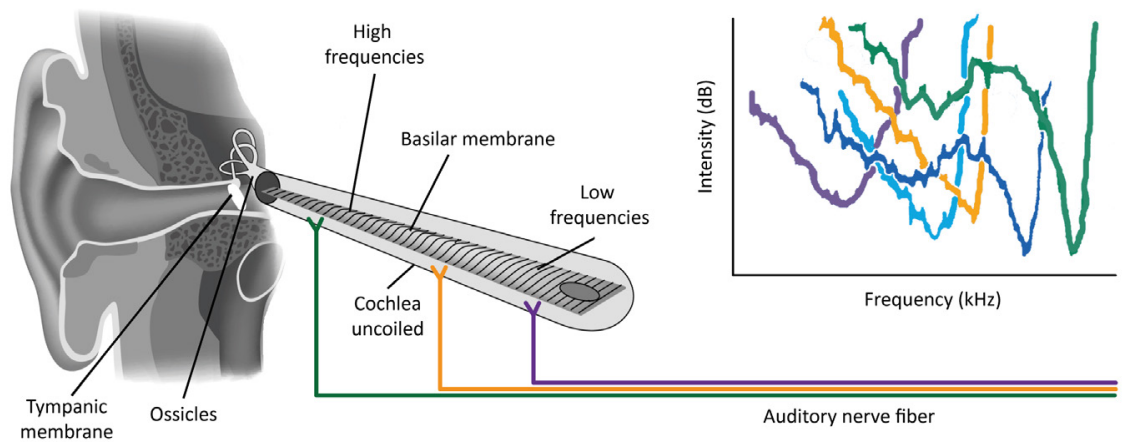

Figure 2. Cochlear transduction and tonotopy. Hair cells in the base of the basilar membrane (green) code for high frequencies; hair cells in the apex (violet) code for low frequencies. Adapted from Kern et al., 2008.

In the spiral ganglion, bipolar neurons send their dendrites to the base of the hair cells while their axons form the auditory portion of the $8^{\text {th }}$ cranial nerve (auditory nerve) and send impulses to the cochlear nucleus, the first auditory nucleus. Three different types of fibers are found in the auditory nerve, considering their spontaneous activity rates (SR): low, medium and high (Liberman, 1978). The spontaneous activity rates are linked to the intensity sensitivity: high spontaneous fibers present low thresholds (the minimum intensity detected) while low spontaneous activity ones have high thresholds (Figure 3).
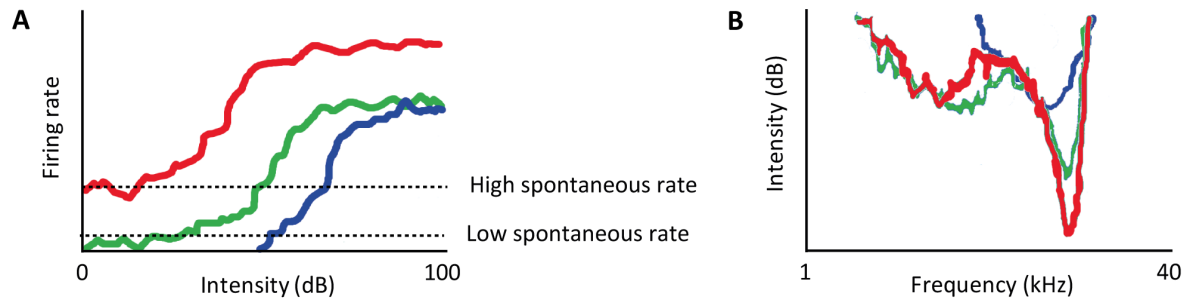

Figure 3. Intensity coding in the auditory nerve. A. Rate-level functions of three different neurons that code for the same frequency. The neuron represented in red shows a high spontaneous rate and a high level sensitivity (low threshold). B. Frequency response areas for the same neurons plotted in A. Low spontaneous rate neurons (blue) present higher thresholds. Adapted from Liberman, 1978. 
Each auditory nerve fiber bifurcates and connects to all three main divisions of the cochlear nuclei, representing the beginning of the parallel auditory processing (Figure 4). Once in the cochlear nucleus, the auditory information is segregated in a series of ascending parallel channels (Cant and Benson, 2003) that reach three main nuclei: the superior olivary complex, the nuclei of the lateral lemniscus and the inferior colliculus (IC).

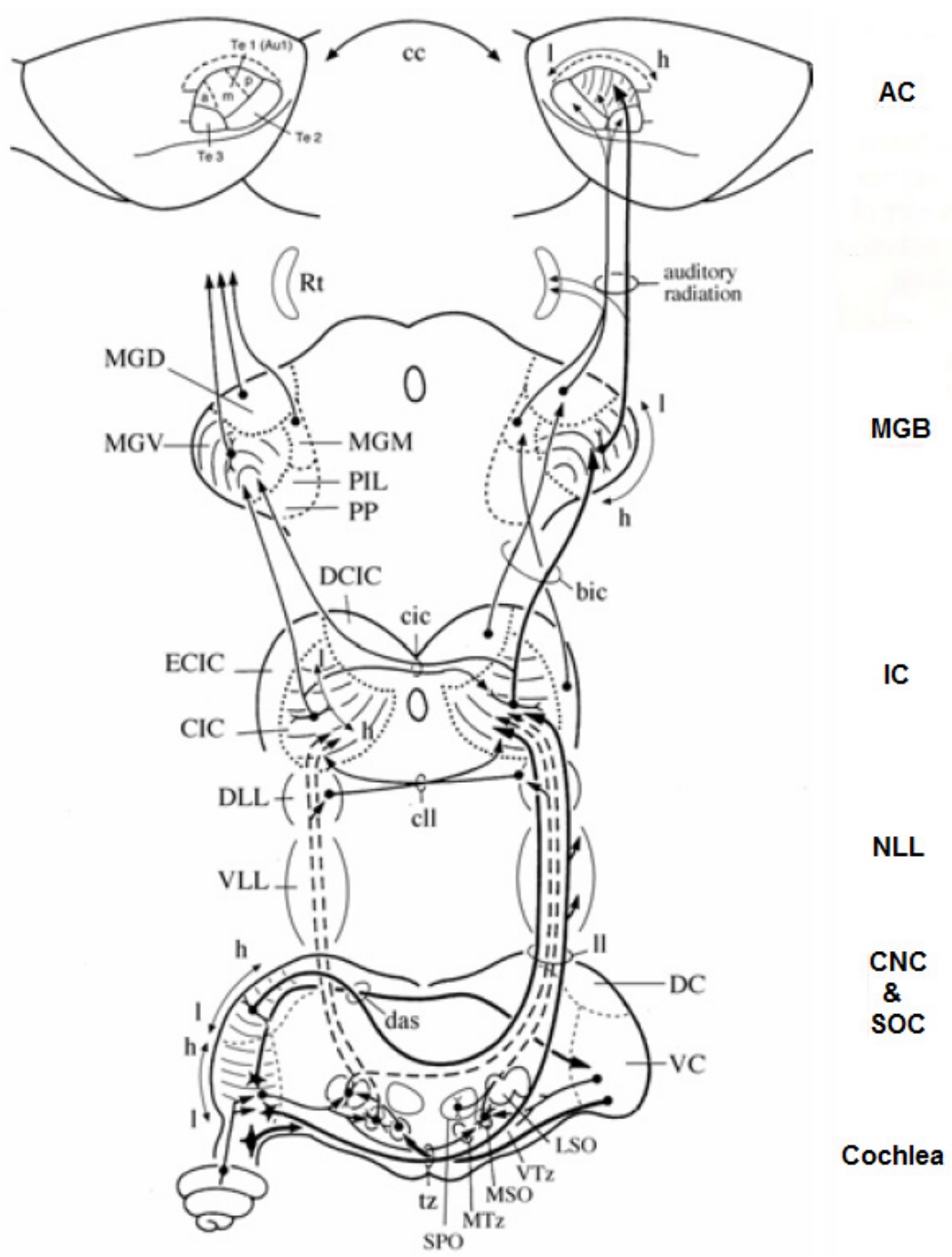

Figure 4. Ascending projections of the auditory pathway. From Malmierca, 2003. 
At the IC all the inputs that have previously diverged converge again (Malmierca, 2003). The IC is the place where new processing takes place and the principal source of ascending inputs to the medial geniculate body (MGB), in the auditory thalamus. At last, the MGB sends its axons to the auditory cortex $(A C)$, the final station of the auditory pathway. Nevertheless, both anatomical and physiological studies have proved obsolete a purely 'bottom-up' processing in the auditory system (Schofield, 2010) by revealing corticofugal and colliculofugal descending projections as well as two olivocochlear systems involved in the control of the cochlea (Figure 5).

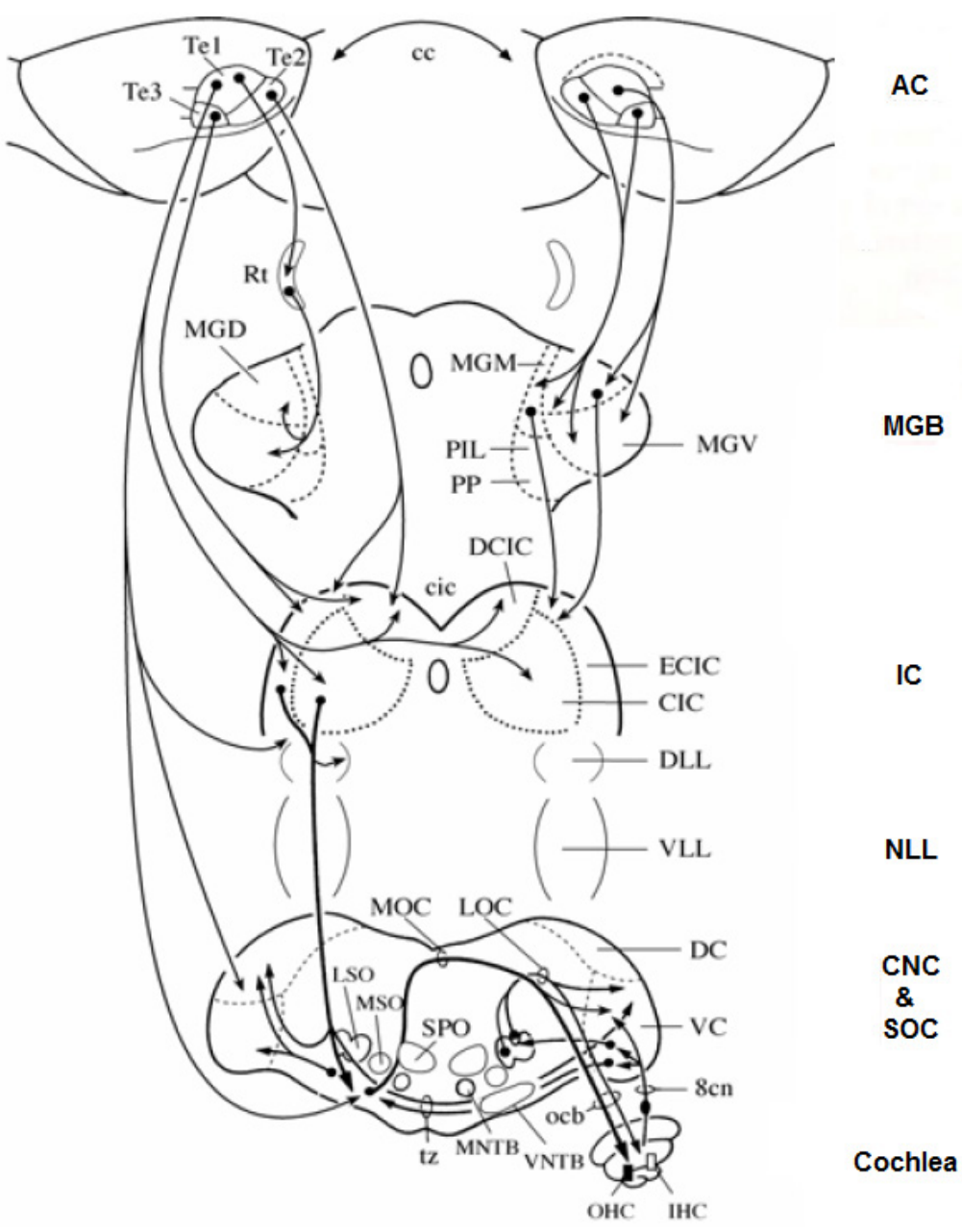

Figure 5. Descending projections of the auditory pathway. From Malmierca, 2003. 
It is still not clear whether the descending auditory pathway should be considered a series of regional feedback loops or simply a descending chain of neurons (Huffman and Henson, 1990). In either case, the role of these loops in audition -and particularly the role of the corticofugal pathway- has been recently linked to gain control and signal filtering (Luo et al., 2008; Robinson and McAlpine, 2009).

The inferior colliculus (IC)

The IC is the principal auditory nucleus in the midbrain, being the nucleus where the inputs from the cochlear nucleus, the superior olivary complex and the lateral lemniscus converge (Malmierca and Hacket, 2010), as well as most of the descending projections from the AC (Lee and Winer, 2005; Winer 2006). Although direct projections from the cochlear nucleus to the MGB have been found (Malmierca et al., 2002; Anderson et al., 2006; Schofield et al., 2014), the high degree of convergence of inputs from the brainstem nuclei into the IC suggests this is a major place of integration of all the brainstem parallel pathways (Malmierca, 2003). The IC is divided into four main regions (Loftus et al., 2008):

- Central nucleus (CNIC): Morphologically, the CNIC is defined by a series of parallel tonotopically organized fibrodendritic laminae (Malmierca and Hacket, 2010) from low- (in the dorsomedial area) to high- (in the ventrolateral area) frequencies (Figure 6A). The dendritic arbors of the neurons in the CNIC are 70-100 $\mu \mathrm{m}$ thick (Figure 6B; Faye-Lund and Osen, 1985; Malmierca et al., 1993). CNIC neurons are under a strong inhibitory modulation by GABA and glycine (LeBeau et al., 2001) and about a $25 \%$ of the neurons in this region are GABAergic (Merchán et al., 2005).

- Dorsal cortex (DCIC): The DCIC is composed by three layers of medium-size multipolar neurons (Figure 6C). The main 
projections to the DCIC arise from lower auditory centers (Malmierca et al., 2005) that encroaches the CNIC as well from intrinsic projections (Saldaña and Merchán, 1992) and bilaterally from the AC (Saldaña et al., 1996).

- $\quad$ Lateral cortex (LCIC): The LCIC covers the CNIC laterally and is mainly composed by medium-sized neurons with large dendritic arbors (Figure 6D; Malmierca et al, 2011) typically aggregated in clusters rich in GABA and acetylcholine (Willard and Ryugo, 1983). The $\mathrm{LCIC}$ receives important inputs from the AC (Saldaña et al., 1996), but also somatosensory inputs (Aitkin et al., 1978, 1981; Malmierca et al., 2003). The LCIC presents multisensory integration and seems to be related to gaze control and with olivocochlear systems (Malmierca and Hacket, 2010).

- Rostral cortex $(\mathrm{RCIC})$ : The $\mathrm{RCIC}$ is located in the rostral portion of the IC and presents both large and medium-sized multipolar neurons (Figure 6E; Faye-Lund and Osen, 1985; Malmierca et al., 1993, 2011). As the $D C I C$ and the $L C I C$, the $R C I C$ receives massive inputs from the AC (Saldaña et al., 1996).

A

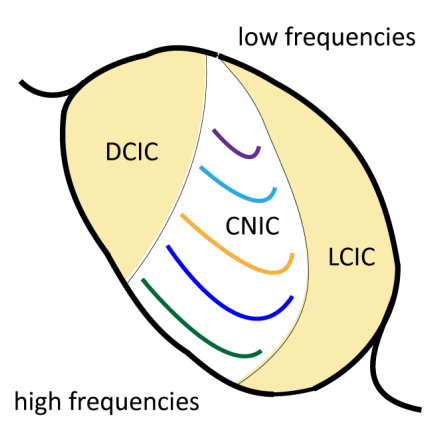

B CNIC

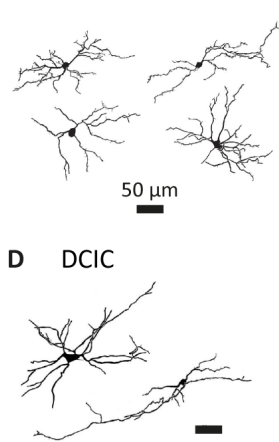

C LCIC

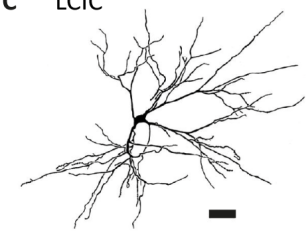

E RCIC

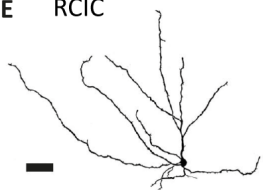

Figure 6. IC morphology and cytoarchitecture. Colored lines in A show the maintained tonotopy in the IC. Adapted from Malmierca et al., 2003. 
The electrophysiological responses of the IC neurons can be described by their spectral (frequency response area, FRA: Figure 7) and temporal properties (peri-stimulus histogram, PSTH, Figure 8). A majority of the neuronal responses in the CNIC present typical V-shaped FRAs among other non V-shaped FRAs, e.g., mosaic, narrow... (LeBeau et al., 2001; Hernández et al., 2005; Palmer et al., 2013). On the other hand, the FRAs in the cortices of the IC are usually broader (Hernández et al., 2005).

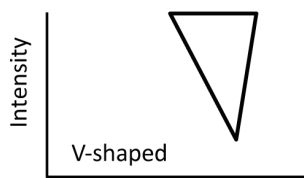

Frequency

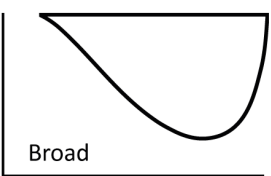

Broad
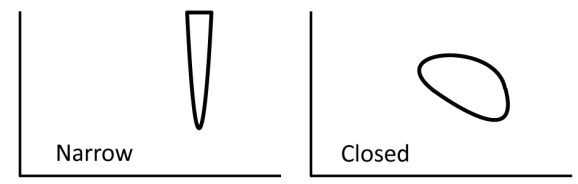

Figure 7. Spectral properties of auditory neurons of the IC.

With respect to temporal properties, neurons of the CNIC usually present sustained, on-sustained, pauser responses (LeBeau et al., 1996; Rees et al., 1997) while the neurons from the cortices of the IC are more likely to present sparse responses with typical onset responses (Li et al., 1998; Lumani and Zhang, 2010). Moreover, neurons in the cortical regions of the IC typically present response latencies longer than the ones found in the CNIC (Lumani and Zhang, 2010). Such delays could be related to particular intrinsic membrane characteristics, due to inhibitory collicular projections or due to the massive descending cortical projection.

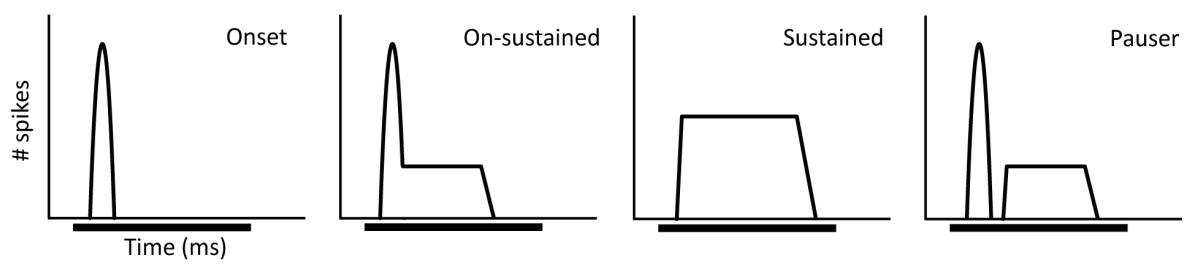

Figure 8. Temporal properties of auditory neurons of the IC. 
The medial geniculate body (MGB)

The MGB is a nuclear complex in the thalamus that has traditionally been considered as a simple relay station for sensory information reaching the AC. Nevertheless, growing evidence suggests that the MGB actively regulates the flow of sensory information and modulates sensory signals (Alitto and Usrey, 2003; Bartlett and Wang, 2007; Sherman, 2007; Yu et al., 2009). One important feature of the auditory thalamus is that GABA is the only neurotransmitter mediating inhibition (Bartlett and Smith, 1999) because MGB lacks glycinergic receptors (Aoki et al. 1988; Friauf et al. 1997). Moreover, as GABAergic interneurons are virtually absent in the rat MGB (only 1\%; Winer and Larue, 1996; Bartlett and Smith, 1999), the two major sources of GABAergic inhibition to the MGB arise from the IC (Winer et al. 1996; Peruzzi et al. 1997; Ito et al. 2011) and the thalamic reticular nucleus (TRN; Rouiller et al. 1985). The MGB is divided in three regions (Winer, 1985):

- $\quad$ Ventral (MGV): MGV neurons present highly oriented dendritic arbors arranged in parallel with the afferent fibers, resulting in fibrodendritic laminae similar to the ones found in the CNIC (Figure 9A; Winer et al., 1999). It is composed by small bi-tufted neurons, i.e., with two polarized dendritic fields (Figure 9D). The main source of inputs come from the CNIC (Bartlett and Smith, 1999), while its axons project to the core (primary area) of the AC.

- $\quad$ Dorsal (MGD): The MGD mainly presents big stellate neurons with radiate dendrites (Figure 9C; Bartlett and Smith, 1999). It is not tonotopically organized and there is no defined cytoarchitecture. Latencies in the MGD are typically longer than in the MGV (Calford, 1983). The main sources of input originate in the DCIC, the LCIC and the AC, while the outputs of the MGD 
terminate directly to belt (secondary areas) of the $A C$ (Malmierca and Hacket, 2010).

- Medial (MGM): The MGB is characterized by the presence of huge magnocellular neurons (Figure 9B) with broad auditory tuning (Aitkin, 1973; Rouiller et al., 1989). The main inputs arise from the LCIC, the cochlear nucleus (Malmierca et al., 2002; Anderson et al., 2006) and the core of the AC, but also from visual and somatosensory structures (Smith and Spirou, 2002). The MGM projects both to the core and belt areas of the $A C$ but it is well known that it also sends direct inputs to the amygdala (Doron and Ledoux, 1999).

A

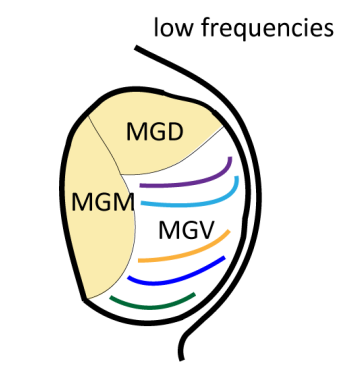

high frequencies
B MGM

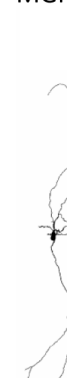

C MGD

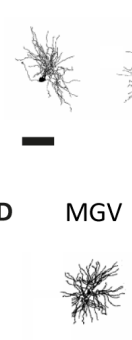

$100 \mu \mathrm{m}$
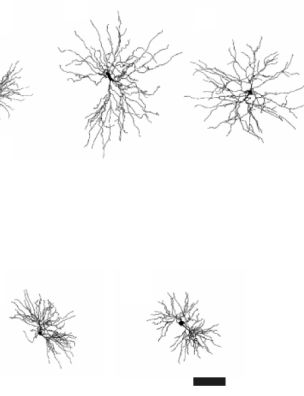

Figure 9. MGB morphology and cytoarchitecture. Colored lines in A show the maintained tonotopy in the MGB. Adapted from Bartlett and Smith, 1999.

The lemniscal and non-lemniscal pathway

Parallel synaptic signaling is a highly conserved neural computational mechanism in the mammalian brain. Across virtually every sensory modality, ascending communication is conducted via two largely segregated channels known as the lemniscal and the non-lemniscal pathways (Hu et al., 1994; Hu, 2003). In the auditory system, the lemniscal pathway arises from the CNIC, ascends to the MGV and projects to core regions of the AC (Figure 9, blue 
lines), while the non-lemniscal system presents tectothalamic projections from the cortices of the IC ( $\mathrm{LCIC}, \mathrm{DCIC}, \mathrm{RCIC})$ to the dorsal (MGD) and medial (MGD) divisions of the MGB, the output of which is relayed to the belt and higher order cortical regions (Figure 10, red lines; Hu et al., 1994; Hu, 2003; Lee and Sherman, 2010; Lee and Sherman, 2011). Both pathways are considered to engage in different aspects of auditory functions. Briefly, the lemniscal pathway processes purely auditory information providing a reliable representation of sound features (de Ribaupierre, 1997), while the nonlemniscal pathway provides context-dependent information (Calford and Aitkin, 1983), multisensory sensitivity (Hu et al., 1994) and reward behavioral conditioning (Komura et al., 2001, 2005).

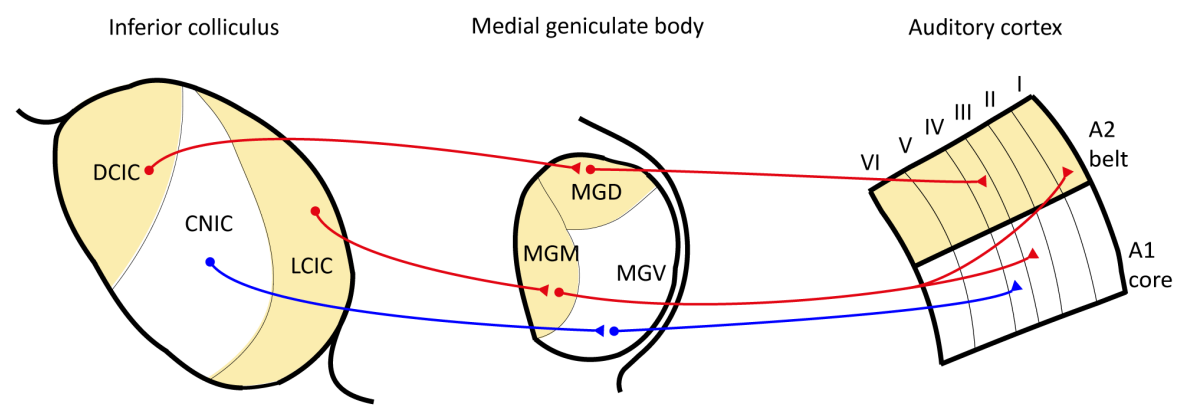

Figure 10. Scheme of the auditory lemniscal and non-lemniscal pathways.

The auditory cortex $(\mathrm{AC})$ is located in the temporal region of the cortex, where the MGB axons terminate, and possesses a characteristic columnar organization, with neurons organized in 6 layers as in other neocortical regions (Malmierca and Hacket, 2010). It is typically divided in a core (also known as primary area) where the frequency is tonotopically represented, surrounded by belt (secondary areas), with a more complex frequency representation (de Ribaupierre, 1997; Polley et al., 2007).

Thalamic projections terminate on layers III and IV of the AC, while the corticofugal projections arise from layers $\mathrm{V}$ and $\mathrm{VI}$ of the $\mathrm{AC}$ (Llano and 
Sherman, 2008) and target the MGB (Winer and Larue, 1987), the IC (Lee and Winer, 2005; Winer 2006), the superior olivary complex (Shneiderman and Henkel, 1987) and the cochlear nucleus (Luo et al., 2008; Liu et al., 2010). Corticofugal projections to the MGB can be direct excitatory synaptic contacts (Winer and Larue, 1987) or via the thalamic reticular nucleus (TRN, Jones, 1975; Rouiller et al., 1985, Harris, 1987), a small GABAergic nucleus that send its axons to the MGB and may be an important site of attentional modulation (Crick, 1984).

\section{Auditory scene analysis and deviance detection}

The main purpose of sensory systems is to aid the survival of their owner in a complex world (Klug and Grothe, 2010). In everyday life, animals are immersed in a continuous flow of sounds generated by multiple sources and the auditory system has to select which acoustic elements are the most relevant and create perceptual constructs (Fishman and Steinschneider, 2010). In order to perform an auditory scene analysis (Bregman, 1990; Winkler et al., 2009) and with the idea of focusing on specific acoustic objects, animals combine both auditory spatial cues and auditory feature cues to separate the sound streams of interest from background environmental noise (Fritz et al., 2007), a process perfectly illustrated with the 'cocktail party effect' (Cherry, 1953).

One way of organizing the acoustic scene is retaining it in form of sound 'objects' (Winkler et al., 2009), store the objects regularity in a sensory memory trace, generate predictions about the forthcoming events and compare the subsequent incoming sounds to these predictions (Bendixen et al., 2012). This is the current theoretical approach for deviance detection (Näätänen et al., 1978), i.e., the detection of new or deviant contextual events in an otherwise monotonous auditory scene. Even though attention can modulate deviance detection (Näätänen et al., 1993) and it is implicated 
in auditory scene analysis (Mesgarani and Chang, 2012), the neuronal basis of deviance detection is clearly pre-attentive (Näätänen et al., 1978; Tiitinen et al., 1994; Schröger and Wolff, 1998).

\section{Mismatch negativity ( $M M N)$}

Deviance detection is a phenomenon well known to occur in humans, first described by Näätänen and colleagues back in 1978. The authors used an auditory event-related potential (Figure 11A) to measure and quantify by the mismatch negativity (MMN, Figure 11B) the ability of the brain to detect rare sounds within a sequence of repetitive sounds. MMN is usually measured with an oddball protocol, where low probability of appearance (deviant) sounds are randomly embedded within sequences of common (standard) sounds. MMN is defined as the difference between the event-related potentials for the deviants (red trace in Figure 11B) and the standards (blue trace in Figure 10B), where the potential generated for the deviant sound is larger than for the standard sound. MMN is sensitive to the stimulus history, its magnitude depends on the probability of the deviant stimuli and could be elicited by sounds that violate some aspect in the regularity of the sequence (e.g., frequency, amplitude, spatial location...).

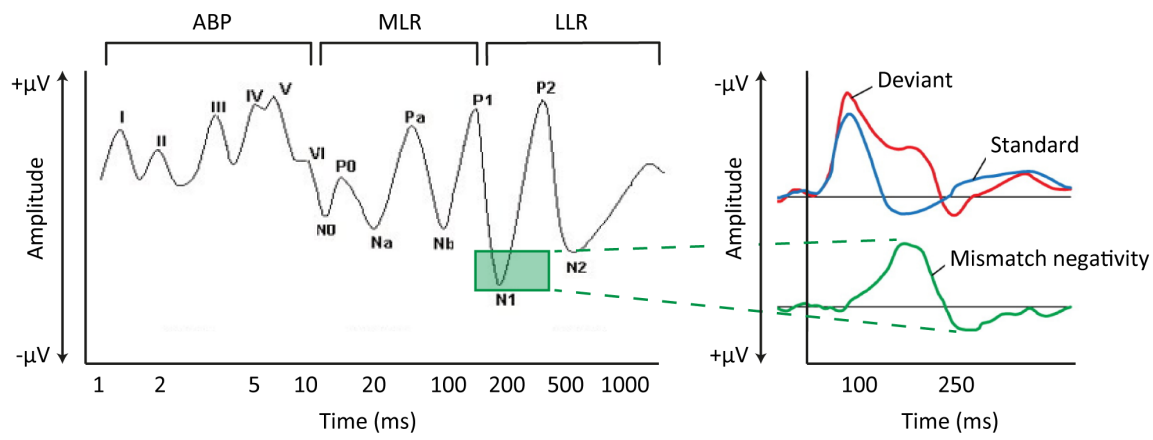

Figure 11. Auditory evoked potentials and mismatch negativity. ABR: auditory brainstem responses. MLR: middle latency responses. LLR: long latency responses. 
MMN peaks around 150-250 ms from stimulus onset, somewhere between the $\mathrm{N} 1$ (100 ms) and the P2 waves (180 ms). MMN can be interpreted as an enhancement of the $\mathrm{N} 1$ wave or as a phenomenon independent of $\mathrm{N} 1$. This differentiation is not trivial because the N1 wave is attributed to basic auditory perceptions from the AC (Hari et al., 1984; Maess et al., 2007) and one of the current interpretations for MMN is that adaptation is related with MMN generation (Fishman, 2013). There are two hypothesis concerning the neural mechanisms and meaning of the MMN:

- Predictive coding: In Fishman's words (2013), "the brain generates a prediction concerning what the next sound in the sequence should be [...] and when properties of the incoming sound fail to agree with the prediction, a prediction 'error' is registered". Thus, the violation of the regularity would depend on sensory memory traces that allow for the generation of signal errors (Näätänen et al., 2001; Friston, 2005) and MMN would be a post hoc phenomenon independent of the N1 wave.

- Neural adaptation: MMN is an adaptation process occurring along the auditory pathway. Again in Fishman's words (2013), MMN would "not reflect a higher-level comparison process or change-detection per se, but rather adaptation to the repeating standard sounds. [...] As (neurons activated by rare deviant sounds) are stimulated less frequently than neurons tuned to the standards, neurons tuned to the deviants ('fresh afferents') are less adapted than those stimulated by the standards". Thus, deviant sounds are going to elicit larger responses because MMN would be related to a specific decrease of the signal (adaptation) elicited for the standard sound by the N1 wave (Jääskeläinen et al., 2004; May and Tiitinen, 2010). 
Interestingly, recent electrophysiological papers (Simpson et al., 2014) point out that auditory scene analysis may be driven by two phenomena: the adjustment of the functional dynamic range of response to the most common stimuli (Dean et al., 2005) and stimulus-specific adaptation (SSA), the ability of some auditory neurons to respond to the less common and rare stimuli while greatly reducing the response to the repeated ones (Ulanovsky et al., 2003).

Stimulus-specific adaptation (SSA)

At the single neuron level, the detection of rare acoustic events is reflected by SSA, whereby neurons adapt to frequently occurring stimuli, but resume firing when 'surprised' by rare sounds (Figure 12). It is widely accepted that SSA lies upstream of MMN generation, regardless of whether MMN is actually reflecting a purelly adapting phenomena. SSA was initially described in the AC (Ulanovsky et al., 2003), but has been later described in the IC (Pérez-González et al., 2005; Malmierca et al., 2009) and the MGB (Anderson et al., 2009, Antunes et al., 2010). So far, the IC is the first auditory station where SSA is found, as this feature has not been found in the cochlear nucleus (Ayala et al., 2013).

The basic properties of SSA in the auditory system have been studied in detail in the AC (Ulanovsky et al., 2003, 2004; Szymanski et al., 2009; von der Behrens et al., 2009; Farley et al., 2010; Taaseh et al., 2011; Fishman and Steinschneider, 2012; Yaron et al., 2012; Briley and Krumbholz, 2013; Nir et al., 2013; Hershenhoren et al., 2014; Klein et al., 2014; Xu et al., 2014) ${ }^{1}$, the auditory thalamus (Anderson et al., 2009; Yu et al., 2009; Antunes et al., 2010; Antunes and Malmierca, 2011; Bäuerle et al., 2011; Richardson et al.,

\footnotetext{
${ }^{1}$ For a general review of SSA and MMN see Escera and Malmierca, 2013; Nelken, 2014 or Malmierca et al, 2014.
} 
2013; Duque et al., 2014) ${ }^{2}$ and the IC (Pérez-González et al., 2005, 2012; Reches and Gutfreund, 2008; Malmierca et al., 2009; Lumani and Zhang, 2010; Reches et al, 2010; Zhao et al., 2011; Duque et al., 2012; Patel et al., 2012; Pérez-González and Malmierca, 2012; Thomas et al., 2012; Ayala et al., 2013; Ponnath et al., 2013; Duque and Malmierca, 2014) ${ }^{3}$.

Interestingly, some differences have been found between cortical and subcortical SSA. SSA is strong in the non-lemniscal subcortical regions (Figure 12A, red dotted areas) while the levels of SSA in the lemniscal areas of the IC (Malmierca et al., 2009) and the MGB (Antunes et al., 2010) are very low or negligible. In contrast, SSA in the AC has been found in the lemniscal region (Ulanovsky et al., 2003; Figure 12A, red dotted area).

Moreover, while neurons exhibiting SSA in the IC (Malmierca et al., 2009) and the MGB (Antunes et al., 2010) tend to be mainly onset responders with short latencies (Figure 12B-C), neurons in the AC exhibit SSA mainly in the sustained part of the neuronal response (Figure 12B-C; Ulanovsky et al., 2003). Nelken and colleagues speculate about the possibility that intra-cortical processing contributed to SSA in the AC. Besides, as SSA in the $A C$ was observed in the core region of the $A C$, some authors proposed that SSA emerges in the $A C$ as a high order feature that can be inherited by the IC and the MGB via the corticofugal pathway (Nelken and Ulanovsky, 2007; Yu et al., 2009). However, recent studies (Antunes and Malmierca, 2011; Anderson and Malmierca, 2013) demonstrate that the corticofugal projection is modulating, but not generating, subcortical SSA. Currently, it is thought that SSA is created de novo at each auditory station or transmitted in a cascade process from low to high order nuclei.

\footnotetext{
${ }^{2}$ For a review of SSA in the MGB see Antunes and Malmierca, 2014.

${ }^{3}$ For a review of SSA in the IC see Ayala and Malmierca, 2013.
} 
A

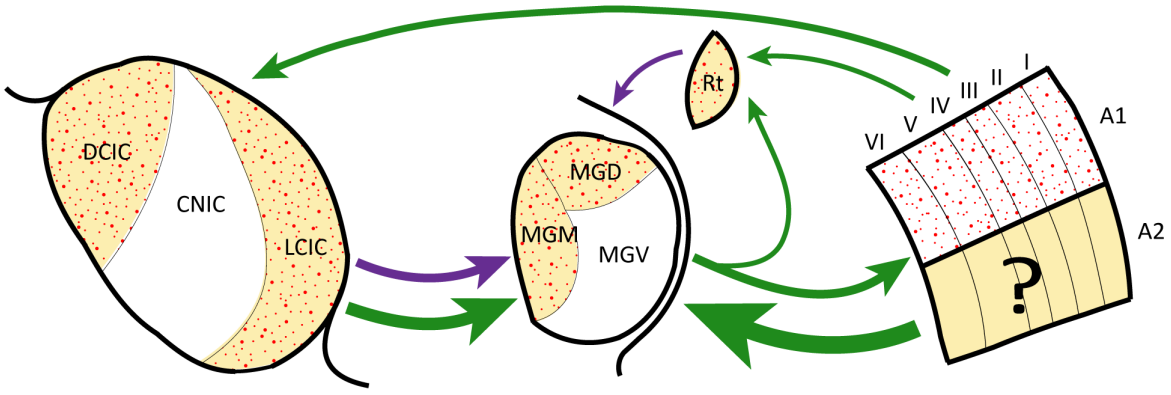

B
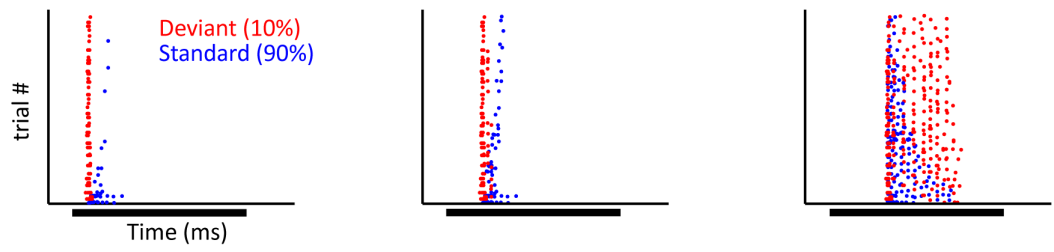

C
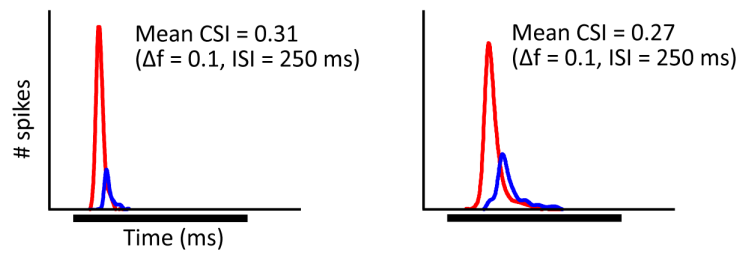

Mean CSI $=0.27$ $(\Delta \mathrm{f}=0.1, \mathrm{ISI}=736 \mathrm{~ms})$

D
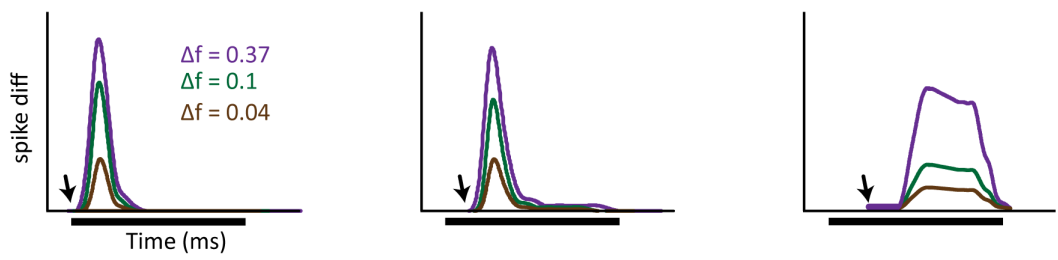

E
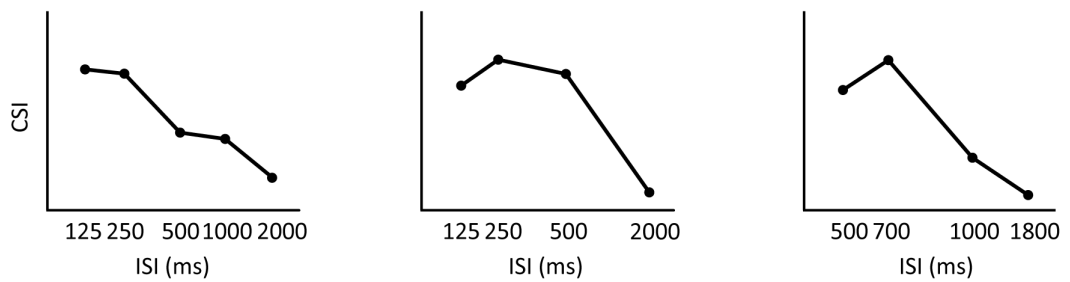

Figure 12. Comparison of SSA between IC, MGB and AC. A. Red dotted areas indicate regions where SSA is strong. Non-lemniscal divisions are highlighted in yellow shaded areas. Green arrows: glutamatergic excitatory projections; purple arrows: GABAergic inhibitory projections. B. Dot raster plots show adapted responses to the standard stimulus (blue dots) while the response to the deviant stimulus (red dots) is not adapted. C. PSTH showing the normalized response from B. D. Relation between the response difference between the deviant and standard stimuli and the frequency contrasts $(\Delta \mathrm{F})$. E. Effect of stimulus repetition rate on the level of SSA. 
Cortical and subcortical SSA is similar in several respects: it is a rapid phenomenon and highly sensitive to 1 ) stimulus statistics (the larger the probability of occurrence of the deviant sound, the larger the adaptation), 2) the frequency contrast (Figure 12D) and 3) the presentation rate (Figure 12E). Nevertheless, SSA can be observed at time intervals as long as $2000 \mathrm{~ms}$ (Ulanovsky et al., 2003; Antunes et al., 2010; Ayala and Malmierca, 2013). Intriguingly, the neuronal mechanisms that create SSA are still unknown. Inhibition can actively modulate SSA (Yu et al., 2009), but does not generate SSA (Pérez-González et al., 2012, Duque et al., 2014). At last, whether SSA is a mechanism depending on the neuronal inputs or it is operating at the output of the neuron is still an open question. 



\section{HYPOTHESIS}



The basic properties of SSA have been studies in detail, but consensus has not been reached about the neuronal mechanisms that encode for SSA and what role, if any, plays inhibition in the generation of SSA. Two main theories have risen in the last decade about the possible neuronal mechanisms that encodes for SSA. On one hand, SSA could depend on intrinsic activity-dependent mechanisms operating at the output of the neuron, at the level of the membrane potential (Abolofia et al., 2011).

On the other hand, SSA could also depend on mechanisms operating at the inputs of the neurons (Ulanovsky et al., 2004; Reches et al., 2010), probably through cumulative input-specific synaptic depression. Such synaptic depression model (Grill-Spector et al., 2006; Briley and Krumbholz, 2013) has risen as the best explanation for SSA and the frequency-specific adaptation channels theory (Eytan et al., 2003; Taaseh et al., 2011) supports this model.

Besides, the role of inhibition in the generation of SSA is still not clear. Some authors (Pérez-González et al., 2012) have described a gain control role for the GABAergic system in the IC, while others (Yu et al., 2009) suggested that the GABA inputs from the TRN to the MGB shape the novelty response. Moreover, almost all the experiments have been performed in anesthetized preparation and there is little doubt that anesthesia may alter some neuronal properties by specifically affecting the inhibitory system, hence altering the excitatory/inhibitory ratio (Rudolph and Antkowiak, 2004).

We therefore hypothesized that:

1. SSA is a mechanism input dependent defined by the width of the frequency channels.

2. SSA is a genuine mechanism not generated by anesthesia that bay be either controlled or generated by inhibition. 

OBJECTIVES 

At the light of the above-mentioned backgrounds, the objectives of this Doctoral Thesis were:

1. To evaluate the frequency and intensity dependence of SSA in the IC (Study I).

2. To observe if SSA is either generated or modulated by the GABAergic system in the MGB (Study II).

3. To test what effect, if any, has anesthesia on SSA (Study III).

4. To determine if SSA can be elicited by purely intensity deviant sounds and to understand the physical properties of the sound that can evoke SSA (Study IV). 

RESULTS 

Study I: Frequency and level dependence of SSA

Duque D, Pérez-González D, Ayala YA, Palmer AR, Malmierca MS (2012)

Topographic distribution, frequency, and intensity dependence of

stimulus-specific adaptation in the inferior colliculus of the rat.

Journal of Neuroscience 32:17762-17774.

doi: 10.1523/JNEUROSCI.3190-12.2012. 



\title{
Topographic Distribution, Frequency, and Intensity Dependence of Stimulus-Specific Adaptation in the Inferior Colliculus of the Rat
}

\author{
Daniel Duque, ${ }^{1}$ David Pérez-González, ${ }^{1}$ Yaneri A. Ayala, ${ }^{1}$ Alan R. Palmer,${ }^{3}$ and Manuel S. Malmierca ${ }^{1,2}$ \\ ${ }^{1}$ Auditory Neurophysiology Unit, Laboratory for the Neurobiology of Hearing, Institute of Neuroscience of Castilla y León, 37007 Salamanca, Spain \\ ${ }^{2}$ Department of Cell Biology and Pathology, Faculty of Medicine, University of Salamanca, 37007 Salamanca, Spain, and ${ }^{3}$ Institute of Hearing Research, \\ Medical Research Council, Nottingham NG7 2RD, United Kingdom
}

\begin{abstract}
The ability to detect unexpected sounds within the environment is an important function of the auditory system, as a rapid response may be required for the organism to survive. Previous studies found a decreased response to repetitive stimuli (standard), but an increased response to rare or less frequent sounds (deviant) in individual neurons in the inferior colliculus (IC) and at higher levels. This phenomenon, known as stimulus-specific adaptation (SSA) has been suggested to underpin change detection. Currently, it is not known how SSA varies within a single neuron receptive field, i.e., it is unclear whether SSA is a unique property of the neuron or a feature that is frequency and/or intensity dependent. In the present experiments, we used the common SSA index (CSI) to quantify and compare the degree of SSA under different stimulation conditions in the IC of the rat. We calculated the CSI at different intensities and frequencies for each individual IC neuron to map the neuronal CSI within the receptive field. Our data show that high SSA is biased toward the high-frequency and low-intensity regions of the receptive field. We also find that SSA is better represented in the earliest portions of the response, and there is a positive correlation between the width of the frequency response area of the neuron and the maximum level of SSA. The present data suggest that SSA in the IC is not mediated by the intrinsic membrane properties of the neurons and instead might be related to an excitatory and/or inhibitory input segregation.
\end{abstract}

\section{Introduction}

The ability to detect unexpected stimuli within the environment is an important function of the brain in general, and of the auditory system in particular, where it mostly depends on some forms of neuronal adaptation (Ulanovsky et al., 2003; Jääskeläinen et al., 2007). The adaptation to repeated sounds while maintaining responsiveness to uncommon ones is known as stimulus-specific adaptation (SSA), and it is thought to be one of the mechanisms that allows novelty detection (Ulanovsky et al., 2003, 2004). SSA was initially described in the auditory cortex (Ulanovsky et al., 2003), but more recently it was found also in the auditory midbrain [inferior colliculus (IC) (Malmierca et al., 2009)] and the thalamus [medial geniculate body (MGB) (Anderson et al., 2009; Yu et al., 2009; Antunes et al., 2010)].

\footnotetext{
Received July 4, 2012; revised Sept. 18, 2012; accepted 0ct. 13, 2012.

Author contributions: A.R.P. and M.S.M. designed research; D.D. performed research; D.D., D.P.-G., and Y.A.A. analyzed data; D.D., D.P.-G., A.R.P., and M.S.M. wrote the paper.

This work was supported by the Spanish Ministerio de Economía y Competitividad (MEC) (BFU2009-07286) Spanish MEC (EUI2009-04083) in the frame of the ERA-NET NEURON (Network of European Funding for Neuroscience Research), to M.S.M. D.D. held a fellowship from the Spanish MEC (BES-2010-035649). D.P.G. held a postdoctoral fellowship from the Botín Foundation. The funders had no role in study design, data collection and analysis, decision to publish, or preparation of this manuscript. The experiments were performed in the Laboratory for the Neurobiology of Hearing, Institute of Neuroscience of Castilla y León, University of Salamanca, Salamanca, Spain. We thank Dr. Nelken and Dr. Caspary for fruitful discussion in a previous version.

Correspondence should be addressed to Manuel S. Malmierca, Institute of Neuroscience of Castilla y León, University of Salamanca, Calle del Pintor Fernando Gallego, 1, 37007 Salamanca, Spain. E-mail: msm@usal.es.

DOI:10.1523/JNEUROSCI.3190-12.2012

Copyright $\odot 2012$ the authors $\quad 0270-6474 / 12 / 3217762-13 \$ 15.00 / 0$
}

The basic properties of SSA have been studied in detail in the auditory cortex (Ulanovsky et al., 2003, 2004; von der Behrens et al., 2009; Taaseh et al., 2011), MGB (Yu et al., 2009; Antunes et al., 2010; Antunes and Malmierca, 2011), and IC (Pérez-González et al., 2005; Malmierca et al., 2009; Lumani and Zhang, 2010; Zhao et al., 2011), but little is known about how it changes within the neuronal receptive field. This is an important issue, since the variation of SSA across the receptive field may shed light on how the neuron's inputs contribute to its generation (Ulanovsky et al., 2004), while a homogeneous SSA would suggest a larger contribution of the intrinsic properties to its origin (Abolafia et al., 2011), although SSA by definition, as opposed to nonspecific adaptation, already suggests that these properties are not involved.

The IC is a key midbrain nucleus that integrates information from all ascending and descending auditory pathways (Malmierca, 2003), and the lowest auditory station where SSA has been found so far. Neurons in the IC project to the auditory cortex via the MGB. Moreover, the IC is the auditory nucleus where the lemniscal and nonlemniscal pathways emerge (for review, see Malmierca, 2003; Lee and Sherman, 2011). This division is relevant because the lemniscal pathway (associated with the central nucleus of the IC) is linked to the processing of basic acoustic features, while the nonlemniscal (lateral, rostral, and dorsal cortices of the IC) (Malmierca et al., 2011) is related to the analysis of more complex features of sound (Hu et al., 1994; Hu, 2003) and multisensory integration (Aitkin et al., 1981; Malmierca et al., 2002). Previous studies on SSA have demonstrated the prevalence of strong SSA in neurons from the nonlem- 


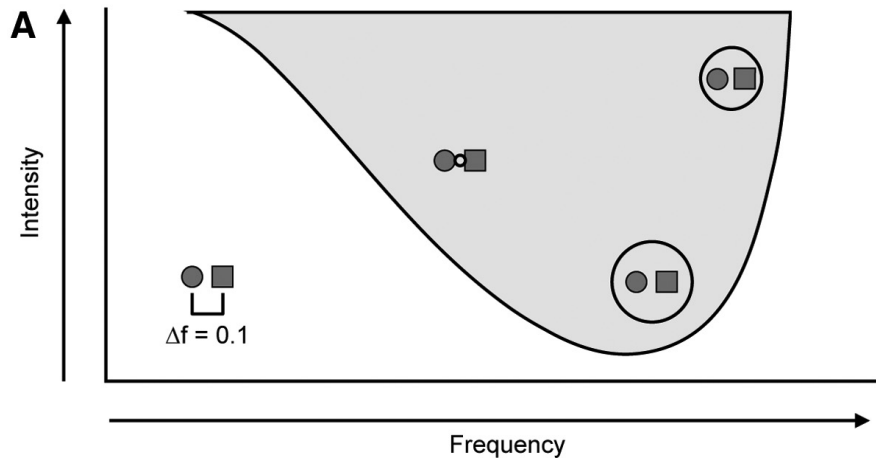

B
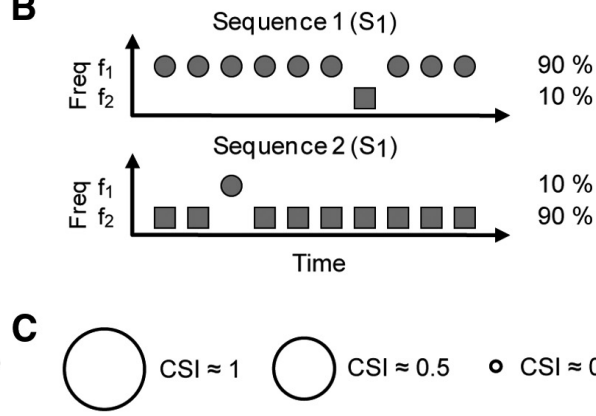

$\mathrm{CSI} \approx 1$

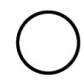

$\mathrm{CSI} \approx 0.5$

- $\mathrm{CSI} \approx 0$

Figure 1. Oddball paradigm and stimulation protocol. $A$, Schematic FRA showing the protocol of the experiments. Different pairs of frequencies ( $f_{1}$, circle; $f_{2}$, square) were selected within the FRA for the oddball paradigm, covering all the range of frequencies and intensities. The frequency contrast $(\Delta f)$ between the two frequencies remained constant at 0.1 ( 0.141 octaves). $B, 0 \mathrm{ddball}$ paradigm. Sequences of 400 pure tones at the two different frequencies were presented, varying the probability of each of the frequencies. In Sequence 1 , the $f_{1}$ (circle) had $90 \%$ probability of occurring, and $f_{2}$ (square) had $10 \%$. In Sequence 2 , the probabilities of $f_{1}$ and $f_{2}$ were reversed. After performing the oddball paradigm a CSI value summarizes the SSA seen at that region of the FRA. C, Schematic representation of the CSI value. On top of each pair of frequencies in the FRA, we draw a circle proportional to the CSI: the bigger the circle is, the higher the SSA.

niscal divisions of the IC and MGB (Malmierca et al., 2009; Antunes et al., 2010; Lumani and Zhang, 2010).

In the present account, we quantified and compared the degree of SSA in single neurons of the rat IC, at multiple intensities and frequency combinations, to map the variations of SSA throughout the neuronal receptive field. Moreover, we investigated whether neurons exhibiting a particular response type are better able to encode for SSA and if the spectral properties of the neuronal response affected the level of SSA. Our results demonstrate that SSA is more strongly expressed in the high-frequency region of the receptive field, at low firing rates, and in ON responders. Preliminary reports have been presented previously (Duque et al., 2010, 2011).

\section{Materials and Methods}

Surgical procedures. Experiments were performed on 33 adult, female rats (Rattus norvergicus, Long Evans) with body weights between 150 and $260 \mathrm{~g}$. All experimental procedures were performed at the University of Salamanca with the approval of, and using methods conforming to the standards of, the University of Salamanca Animal Care Committee. Anesthesia was induced ( $1.5 \mathrm{~g} / \mathrm{kg}$, i.p., $20 \%$ solution) and maintained $(0.5$ $\mathrm{g} / \mathrm{kg}$, i.p., given as needed) with urethane. Urethane was chosen as an anesthetic because its effects on multiple aspects of neural activity, including inhibition and spontaneous firing, are known to be less than those of barbiturates and other anesthetic drugs (Hara and Harris, 2002). The respiration was maintained artificially (SAR-830/P Ventilator), monitoring the end-tidal $\mathrm{CO}_{2}$ level (CapStar-100). For this purpose, the trachea was cannulated and atropine sulfate $(0.05 \mathrm{mg} / \mathrm{kg}$, s.c. $)$ was administered to reduce bronchial secretions. Details of surgical procedures have been described previously (Pérez-González et al., 2005; Malmierca et al., 2009; Antunes et al., 2010). Body temperature was maintained at $38 \pm 1^{\circ} \mathrm{C}$ by means of a heating blanket. The animal was placed in a stereotaxic frame in which the ear bars were replaced by hollow speculae that accommodated a sound delivery system, inside a sound-sealed room.

Acoustic stimuli and electrophysiological recording. A craniotomy was performed to expose the cerebral cortex overlying the IC. Extracellular single-unit responses were recorded using a tungsten electrode (1-2 $\mathrm{M} \Omega$ ) (Merrill and Ainsworth, 1972) lowered through the cortex by means of a piezoelectric microdrive (Burleigh 6000 ULN). Neuron location in the IC was based on stereotaxic coordinates, physiological criteria of tonotopicity and response properties (Malmierca et al., 2003; Hernández et al., 2005; Pérez-González et al., 2005, 2006; Malmierca et al., 2009), and confirmed histologically afterward.

Acoustic stimuli were delivered through a sealed acoustic system (Malmierca et al., 2009) using two electrostatic loudspeakers (TDT EC1;
Tucker Davis Technologies) driven by two TDT ED1 modules. The stimuli were presented contralaterally to the recording side; search stimuli were pure tones or noise bursts monaurally delivered under computer control using TDT System II hardware and custom software (Faure et al., 2003; Pérez-González et al., 2005, 2006; Malmierca et al., 2008). The output of the system at each ear was calibrated in situ using a $1 / 4$ inch condenser microphone (model 4136; Brüel and Kjær) and a DI-2200 spectrum analyzer (Diagnostic Instruments). The maximum output of the TDT system was flat from 0.5 to $4 \mathrm{kHz}(\sim 110 \pm 7 \mathrm{~dB} \mathrm{SPL})$, from 4.5 to $14.5 \mathrm{kHz}$ ( $\sim 90 \pm 6 \mathrm{~dB}$ SPL), and from 15.5 to $40 \mathrm{kHz}(\sim 95 \pm 7 \mathrm{~dB}$ SPL), presenting a notch at $15 \mathrm{kHz}$. The highest frequency produced by this system was limited to $40 \mathrm{kHz}$. The second and third harmonic components in the signal were $\leq 40 \mathrm{~dB}$ below the level of the fundamental at the highest output level (Malmierca et al., 2008, 2009).

Action potentials were recorded with a BIOAMP amplifier (Tucker Davis Technologies), the $10 \times$ output of which was further amplified and bandpass filtered (TDT PC1; $f_{\mathrm{c}}, 500 \mathrm{~Hz}$ and $3 \mathrm{kHz}$ ) before passing through a spike discriminator (TDT SD1). Spike times were logged with a resolution of $\approx 150 \mu$ s on a computer by feeding the output of the spike discriminator into an event timer (TDT ET1) synchronized to a timing generator (TDT TG6). Stimulus generation and on-line data visualization were controlled with custom software. Spike times were displayed as dot rasters sorted by the acoustic parameter varied during testing.

From an isolated neuron, the approximate frequency tuning was audiovisually determined by presenting pure tones lasting $75 \mathrm{~ms}$ with a $5 \mathrm{~ms}$ rise/fall time (Hernández et al., 2005). We obtained the frequency response area (FRA), the combination of frequencies and intensities capable of evoking a response, as an estimation of the neuronal receptive field. For that, we presented multiple combinations of frequency and intensity using an automated procedure with 5-10 stimulus repetitions at each frequency (from 0.5 to $40 \mathrm{kHz}$, in $20-30$ logarithmic steps, presented randomly) and intensity (10 $\mathrm{dB}$ steps, presented from lower to higher intensities). The spike counts evoked at each combination of frequency and intensity were then plotted using MATLAB. We used this representation of the FRA to calculate the minimum threshold and best frequency (BF) of response, i.e., the frequency where the minimum threshold is found.

Stimulus presentation paradigms. The representation of the FRA allowed us to choose pairs of frequencies $\left(f_{1}\right.$ and $\left.f_{2}\right)$ within the response area of the neuron that elicited a similar firing rate at the same intensity level (Fig. $1 \mathrm{~A}$ ). Both frequencies in each pair were always presented at the same sound level. For each pair of frequencies, stimuli were presented in an oddball paradigm similar to that used to record mismatch negativity responses in human (Näätänen, 1992) and animal (Ulanovsky et al., 2003, 2004; Malmierca et al., 2009; Antunes et al., 2010) studies. Briefly, a train of 400 stimuli containing both frequencies $f_{1}$ and $f_{2}$ was presented 

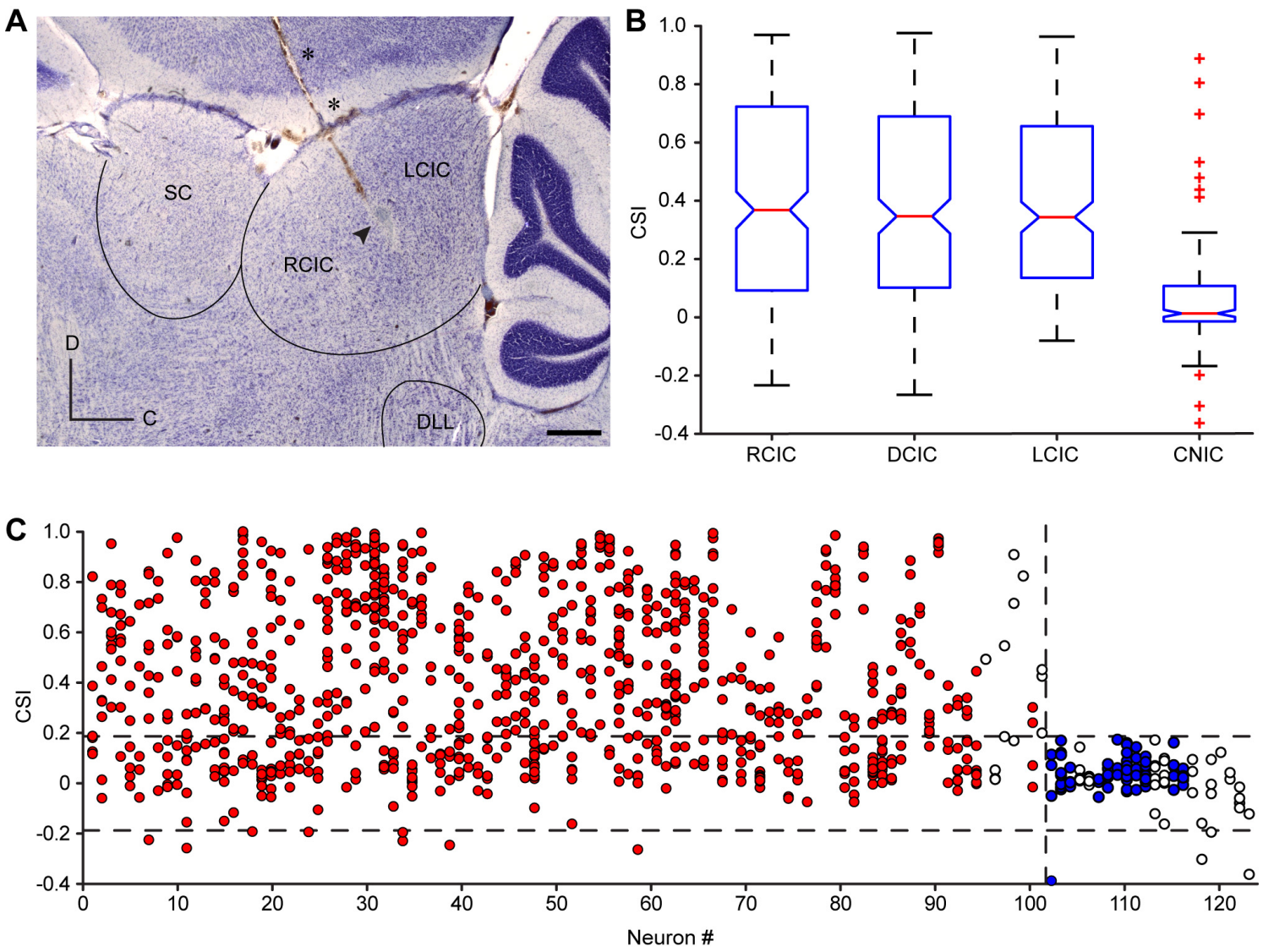

Figure 2. Anatomical location of CSI. A, Photomicrography showing a sagittal section of the IC with a typical electrode track (asterisks) and the electrolytic lesion generated (arrowhead). Scale bar, $500 \mu \mathrm{m}$. C, Caudal; D, dorsal. B, Box plot with the median value (red line) of (SI sorted by anatomic regions. The blue box delimits the 25th and 75th percentiles, and dashed lines show the most extreme data points not considered outliers. Red crosses indicate outliers. Cortical regions (RCIC, DCIC, and LCIC) are significantly different from the CNIC (Kruskal-Wallis test, $p<0.001$ ). C, Neuronal CSI variability. Each dot illustrates the level of CSI for a given pair of frequencies. Any single neuron can present levels of CSI close to 1 and below 0 , depending on the region of the FRA. Red dots are values from neurons that present adapting pairs of frequencies, blue dots are values from neurons that only have nonspecifically adapting pairs of frequencies, and empty dots are pairs of frequencies in identified CNIC neurons. The top dashed line shows the higher cutoff value (0.18), the bottom one shows the lower cutoff value $(-0.18)$, and the vertical dash line illustrates the separation between adapting and nonspecifically adapting neurons. SC, Superior colliculus; DLL, dorsal lateral lemniscus.

under the oddball paradigm: one frequency $\left(f_{1}\right)$ was presented as the standard ( $90 \%$ occurrence), while, interspersed randomly among the standards, the deviant stimuli ( $10 \%$ occurrence) were presented at the second frequency $\left(f_{2}\right)$. After obtaining one data set, the relative probabilities of the two stimuli were reversed, with $f_{2}$ as the standard and $f_{1}$ as the deviant (Fig. 1 B). Dot raster plots (see Fig. 3) are used to illustrate the responses obtained to the oddball paradigm, plotting individual spikes (red dots indicate responses to the deviant; blue dots indicate responses to the standard). Stimulus presentations are marked along the vertical axis. The responses to the standard and deviant stimuli were expressed as spikes per stimulus, to account for the different number of presentations in each condition, due to the different probabilities. The frequency contrast remained constant under all the experimental conditions at $\Delta f=$ 0.10, where $\Delta f=\left(f_{2}-f_{1}\right) /\left(f_{2} \times f_{1}\right)^{1 / 2}$. (Ulanovsky et al., 2003, 2004; Malmierca et al., 2009). This value is equivalent to a frequency separation of 0.141 octaves. The stimuli were presented at a repetition rate of $4 \mathrm{~Hz}$. These conditions have been shown previously to evoke strong SSA in the IC (Malmierca et al., 2009).

The degree of SSA was quantified by calculating the common SSA index (CSI) from the responses (firing rate) elicited in the oddball condition. The CSI has been used in previous studies (Ulanovsky et al., 2003, 2004; Malmierca et al., 2009; Antunes et al., 2010) and is defined as CSI = $\left[\mathrm{d}\left(f_{1}\right)+\mathrm{d}\left(f_{2}\right)-\mathrm{s}\left(f_{1}\right)-\mathrm{s}\left(f_{2}\right)\right] /\left[\mathrm{d}\left(f_{1}\right)+\mathrm{d}\left(f_{2}\right)+\mathrm{s}\left(f_{1}\right)+\mathrm{s}\left(f_{2}\right)\right]$, where $\mathrm{d}(f)$ and $\mathrm{s}(f)$ are responses to each frequency $f_{1}$ or $f_{2}$ when they were the deviant (d) or standard (s) stimulus, respectively. This index reflects the extent to which the response to the standard was suppressed or the response to the deviant was enhanced (Fig. 1C). The possible range of CSI values is from -1 to +1 , being positive if the response to the deviant stimulus is greater. To avoid including the spontaneous activity in the analysis, we set time windows chosen individually in each case. We had a default time window that embraced the whole stimulus ( 10 to $85 \mathrm{~ms}$ ) for low spontaneous activity responses (see Fig. $3 B, C$ ) and, in those cases where the spontaneous rate was higher (see Fig. $3 A, D$ ), it was possible to set the boundaries based on the shape of the peristimulus time histogram (PSTH). When probing for SSA at different frequencies and intensities, we started to collect the data from the lowest intensity at BF and then gradually chose different pairs of frequencies covering (1) frequencies lower and higher than the BF and (2) all the possible range of intensity levels, in $10 \mathrm{~dB}$ steps. There is no a priori octave spacing when choosing the pairs, but we always try to test at both the low- and the high-frequency edges of the FRA, choosing two stimuli that evoked similar firing rates to ensure that all differences in response were due to the statistics of the stimulus ensemble.

Histological verification and recording sites. Each track was marked with electrolytic lesions (10-15 $\mu \mathrm{A}$ for $10-15 \mathrm{~s})$ for subsequent histological localization of the neurons recorded (Fig. $2 A$ ). At the end of each exper- 
iment, the animal was given a lethal dose of sodium pentobarbital and perfused transcardially with PBS $\left(0.5 \% \mathrm{NaNO}_{3}\right.$ in PBS $)$ followed by fixative (a mixture of $1 \%$ paraformaldehyde and $1 \%$ glutaraldehyde in rat Ringer's solution). The brain tissue was sectioned in the sagittal plane into $40-\mu \mathrm{m}$-thick sections that were Nissl stained with $0.1 \%$ cresyl violet. Recording sites were marked on standard sections from a rat brain atlas (Paxinos and Watson, 2005), and units were assigned to one of the four main divisions of the IC (Malmierca et al., 1993, 1995, 2011; Loftus et al., 2008): lateral cortex (LC), rostral cortex (RC), dorsal cortex (DC), or central nucleus $(\mathrm{CN})$. This information was complemented and confirmed by the stereotaxic coordinates used during the experiment to localize the IC.

\section{Results}

To study how SSA varies within the receptive field of IC neurons, we recorded the responses from 124 well-isolated single units throughout the IC while presenting stimuli in an oddball paradigm. For all neurons, we determined the FRA and used the oddball paradigm choosing several pairs of frequencies at different intensities within the FRA (Fig. $1 A$ ). We used a constant frequency contrast $(\Delta f=0.1)$, repetition rate $(4 \mathrm{~Hz})$, and probability of occurrence of the deviant stimuli (10\%).

\section{SSA is better encoded in the nonlemniscal pathway}

We localized histologically 81 of the 124 recorded neurons $(65.3 \%)$ to the different IC subdivisions (Fig. 2). Twenty-five of them were from the rostral cortex (RCIC, $31 \%$ ), 21 from the lateral cortex (LCIC, 26\%), and 17 from the dorsal cortex (DCIC, $21 \%)$. Eighteen were localized to the central nucleus (CNIC, $22 \%$ ). The Kruskal-Wallis one-way ANOVA by ranks shows that the distributions of CSI values obtained in the cortical regions were similar among them (median \pm interquartile range, DCIC, $0.367 \pm 0.629$; RCIC, $0.346 \pm 0.587$; LCIC, $0.343 \pm 0.520$ ), but significantly different from those of the CNIC $(0.012 \pm 0.116$; $p=1.35 \cdot 10^{-13}$; Dunn's method confirmed differences between each cortical region and the CNIC, $p<0.05$ in all cases; Fig. $2 B$ ). Although the level of SSA in the CNIC is distinctly low, seven pairs of frequencies from neurons histologically located in the edges of the CNIC exhibited significant SSA (CSI, >0.4; Fig. 2 B). Since our sample is consistent and biased to the cortical regions of the IC (almost the $80 \%$ of the neurons were localized in cortical regions), in the subsequent analysis we have included all the recorded neurons instead of only the 81 localized histologically, and we will no longer consider regional subdivisions.

\section{SSA varies within the FRA}

From the 124 recorded neurons, we obtained a total of 1057 different pairs of frequencies. On average, we tested nine pairs of frequencies per neuron (range, 3-24 pairs per neuron). Pairs of frequencies that did not respond to both stimuli in the oddball paradigm $(n=68)$ were excluded from the analysis. Figure $2 C$ shows the individual variability of the CSI value for all the neurons in our sample. Since our main goal was a detailed analysis of neurons that exhibit SSA, we established a cutoff to prevent the neurons lacking SSA (as those located in the CNIC) from masking or averaging out the relationship between SSA sensitivity and other response properties under study. We used a CSI value of +0.18 as threshold for significant SSA, since the intrinsic variability of the response could evoke positive values of CSI that are not related to a specifically stronger response to the deviant stimulus. This cutoff value was established previously by Antunes et al. (2010) by choosing the most negative CSI value in the data set $(-0.18)$ to represent the most extreme variance due to random fluctuations in spike counts. We calculated the cutoff value for the present data set obtaining a value of -0.21 , but we considered that the different cutoff values obtained are due to the experimental variability rather than a genuine difference. Thus, for consistency we use the same value as used previously. We consider the range of CSI between -0.18 and +0.18 as an indication of a lack of stimulus-specific adaptation. Thus, a CSI value above 0.18 indicates significant SSA. The few values in our data set below -0.18 (11 of $1057,1 \%$ ) are considered outliers. Neurons that presented all their CSI values below 0.18 are referred to as "nonspecifically adapting" neurons. One-hundred and two neurons (82.3\%; 900 pairs of frequencies) showed high SSA levels (CSI, $>0.18$ ) for at least one of the pairs of frequencies analyzed. The remaining 22 neurons $(17.7 \%)$ showed a low SSA (CSI, $\leq 0.18$ ) for all pairs of frequencies analyzed ( 89 pairs of frequencies). Interestingly, 12 of the 18 neurons that we localized in the CNIC presented such low SSA (Fig. 2C, empty dots).

Figure 3 shows four examples of individual neurons with their corresponding FRA. For each neuron, we display dot raster plots obtained using the oddball paradigm with a pair of frequencies that show strong adaptation (left columns) and with a pair that do not show adaptation (right columns). The corresponding PSTHs are also shown. To assess the variability of SSA within the FRA, we plotted the pairs of frequencies tested (represented by dots in the FRA) and the level of SSA of each pair of frequencies (represented by a circle, with the diameter proportional to the CSI value; Fig. $1 C$ ). Figure $3 A$ shows a broadly V-shaped FRA where 13 pairs of frequencies were tested. Figure $3 B$ shows a multipeaked FRA (18 pairs of frequencies), while Figure $3 C$ shows a narrow FRA (9 pairs of frequencies), and Figure 3D a mosaic FRA (8 pairs of frequencies). In all these cases, the PSTHs exhibit a larger response to the deviant sounds (red line), showing that the neuron presents SSA to some pairs of frequencies (left columns) and a similar response for both conditions when it is not differentially adapted (right columns).

To see how CSI values are distributed throughout the whole auditory receptive field of the rat, we first merged the firing rates of all the FRAs to create a cumulative receptive field (Fig. 4A-D). This cumulative FRA was created over the whole range of frequencies (from 0.5 to $40 \mathrm{kHz}$ ) and intensities (between -20 and $80 \mathrm{~dB}$ SPL) that we tested. The resulting cumulative receptive field obtained was a broadly tuned, V-shaped-like FRA that presented the lowest threshold $(-20 \mathrm{~dB}$ SPL $)$ at $\sim 12 \mathrm{kHz}$. Then, we plotted the center frequency $\left[\mathrm{CF}=\left(f_{2} \times f_{1}\right)^{1 / 2}\right]$ of each pair of frequencies on top of the cumulative receptive field, grouped according to the level of SSA that they elicited, as shown in Fig. 4. For convenience, we established four different groups: CSI < $0.18(n=284$; Fig. $4 A), 0.18<$ CSI $<0.5(n=277$; Fig. $4 B)$, $0.5<\mathrm{CSI}<0.75(n=183$; Fig. $4 C)$, and CSI $>0.75(n=156$; Fig. $4 D$ ). The distribution of the frequencies analyzed with low CSI values (CSI $<0.18$; Fig. $4 A$ ) was spread throughout the entire FRA, covering the whole range of frequencies and intensities used, but a majority of pairs of frequencies tended to be located at the high-intensity regions of $\sim 10-12 \mathrm{kHz}$, which are also the highest firing rate regions (yellow and red areas). In contrast, the highest CSI values ( $>0.75$; Fig. $4 D$ ) were concentrated in the lower firing rate areas (dark blue), which are located at low and medium intensities around the $10-12 \mathrm{kHz}$ region, and also at high frequencies. It is important to note that there were no pairs with CSI values $>0.75$ found in the low-frequency tail of the "synthetic" receptive field. Medium CSI values $(0.18<\mathrm{CSI}<0.75$; Fig. $4 B, C)$ show an intermediate localization. In this case the distribution of the pairs was not as homogeneous as the one 

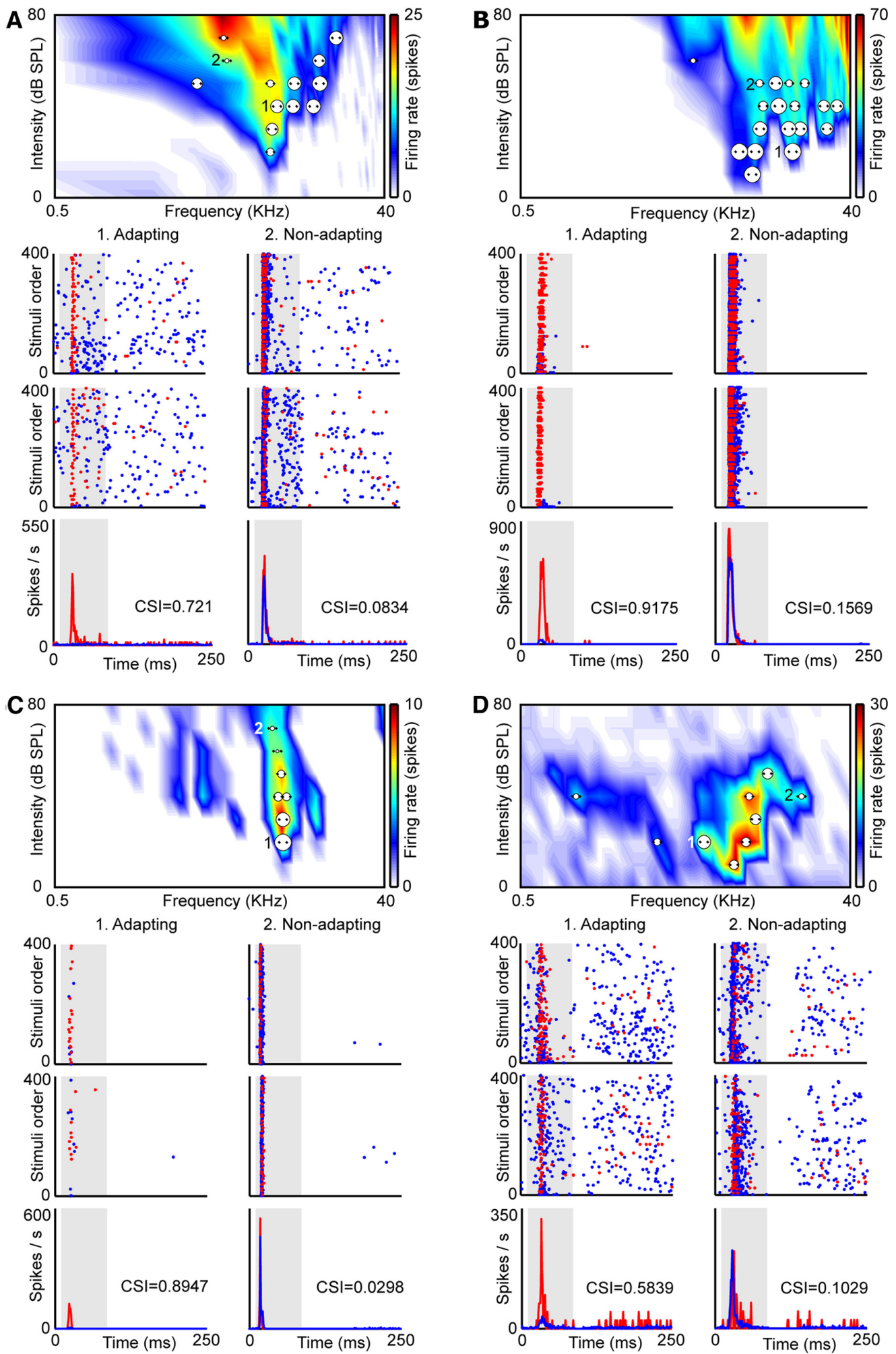

2. Non-adapting
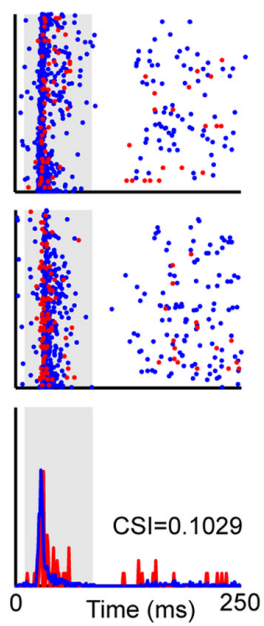

Figure 3. Examples of neurons. $\boldsymbol{A}-\boldsymbol{D}$, Each panel shows the FRA (broad V-shaped FRA, $\boldsymbol{A}$; multipeaked FRA, $\boldsymbol{B}$; narrow FRA, $\boldsymbol{C}$; mosaic FRA, $\boldsymbol{D}$ ) of a neuron and all the pairs of frequencies analyzed as dots. Each pair of dots is associated to a circle the size of which is proportional to the level of CSI evoked. Examples of an adapting pair of frequencies are marked as 1 , (Figure legend continues.) 
observed with low CSI levels, but instead began to show a trend toward low firing rates.

To further analyze the parameters that could affect the strength of SSA, we divided our data into different groups according to the two main sound parameters (i.e., intensity and frequency). First, we created different groups taking into account sound level (decibels of SPL; Fig. 4E): the results showed an emerging trend such that SSA is higher at low sound intensities. Next, we analyzed how the SSA of the IC neurons varied as a function of the sound frequency that we used for stimulation (from 0.5 to $40 \mathrm{kHz}$ ). For this purpose, we calculated the center frequency for each pair of frequencies and arranged them in 0.5octave-wide groups. As shown in Figure $4 F$, high CSI values are clearly skewed toward high frequencies. We finally merged all the information relative to CSI and plotted it together, taking into account the intensity (in decibels of SPL) and the frequency of each pair of frequencies analyzed (in kilohertz) to understand how SSA varies as a function of these two parameters (Fig. 4G). High and low SSA levels are differently distributed. Neurons exhibit the highest CSI values in the regions with frequencies above $10 \mathrm{kHz}$ at low intensities and also at relative high intensities at high frequencies (Fig. 4G, yellow-red). In contrast, the lowest values of CSI (Fig. $4 G$, green-blue) are confined to high intensities and to low frequencies.

At this juncture, to better visualize where the SSA is strongest within the FRA, we replotted the graph in Figure $4 G$, but this time considering the distances relative to the threshold (reTh) and the best frequency of each neuron. In Figure $5 A$, we clearly observe that the highest CSI values are concentrated at the highfrequency edge. To check that we do not have any bias due to high-frequency neurons, we standardized the data to the highfrequency edge (Fig. 5B). This analysis confirms that the results are genuine and not due to a data sampling bias. This plot reinforces the idea that larger CSI values are found at high frequencies and low intensities within the FRA of individual neurons, demonstrating that SSA is not a property homogeneously distributed within the FRA of the neuron.

With the aim of quantifying these effects, we performed a three-way ANOVA, where the three factors evaluated were the frequency, the intensity, and the effect of the neuron (as a random factor). The results of this analysis showed that intensity $\left(F=112.75 ; p=7.18 \cdot 10^{-44}\right)$, frequency $(F=52.25 ; p=$ $\left.4.69 \cdot 10^{-22}\right)$, and neuron $\left(F=9.33 ; p=4.01 \cdot 10^{-79}\right)$ are major factors that shape the level of CSI.

\section{SSA as a function of sound intensity}

To understand how SSA varies through the intensity range, we established six different groups in $10 \mathrm{~dB}$ steps above each neuron's minimum threshold, and we calculated the median CSI

\footnotetext{
(Figure legend continued.) and examples of a nonspecifically adapting pair of frequencies are marked as 2. Below the FRA we show the dot raster plots obtained in the adapting pair (left) and in the nonspecifically adapting pair (right). The blue dots represent spikes evoked by the standard stimulus ( $90 \%$ probability), while the red dots represent those evoked by the deviant stimulus (10\% probability). Stimulus presentations are accumulated in the temporal domain in the vertical axis. In the adapting examples, red dots are more visible because of the specific decrease of the response to the standard stimulus. The top panels are the ones obtained after using Sequence 1 in the oddball paradigm; middle panels are the ones obtained after Sequence 2 under the oddball paradigm. Bottom panels are the PSTHs, averaged for both frequencies when deviant (red) or standard (blue). CSI values obtained in each pair of frequencies are showed as insets in the PSTHs. The shaded backgrounds in the dot raster and PSTH plots indicate the duration of the stimulus.
}

value in each group separately (Fig. $6 \mathrm{~A}$ ). The maximum intensity that we played was $+70 \mathrm{~dB}$ reTh. Thus, we pooled together the +60 and the $+70 \mathrm{~dB}$ reTh groups to make the high-intensity group more reliable. CSI values close to the threshold are significantly larger than those at higher intensity levels (Kruskal-Wallis test, $p<0.001)$. Post hoc comparisons confirmed that all groups were significantly different to those at least $20 \mathrm{~dB}$ apart (Dunn's method, $p<0.05$; Fig. $6 A$ ).

In an attempt to understand the correlation between the intensity level and the firing rate, we performed an additional analysis where we considered that the effect of the intensity might be due to firing rate dependence. For this reason, we took the frequency pairs above $70 \mathrm{~dB}$ SPL and sorted them in three groups according to the firing rate observed in the region of the FRA where we choose the pairs: low ( $<1.5$ spikes/stimulation), middle (1.5 to 4 spikes/stimulation), and high ( $>4$ spikes/stimulation) firing rates. A Kruskal-Wallis test showed that there are no significant differences between the groups (low firing rate, 0.200; middle firing rate, 0.141; high firing rate, $0.098 ; p=0.516$ ). Thus, lower CSI levels at high intensities are independent of the firing rate.

To analyze the relationship between sound level and the latency of the neuronal response, we plotted the latency differences between the standard and the deviant stimuli (defined as the difference between the median first spike latency to the standard and the deviant condition for each frequency) as a function of the different intensity groups that we previously established (Fig. $6 B)$. The results demonstrate that there were no significant differences between the intensity groups (Kruskal-Wallis test, $p=$ 0.172; Table 1).

To study the time course of adaptation in the IC population, we examined how responses changed over the 400 consecutive trials. Figure $6 C$ shows the average population response as a function of trial number for both standard (blue trace) and deviant stimuli (red trace). In the three groups of intensities analyzed (low intensities, $\leq 20 \mathrm{~dB}$ reTh, Fig. $6 \mathrm{C}$, left; medium intensities, $30-40 \mathrm{~dB}$ reTh, middle; high intensities, $\geq 50 \mathrm{~dB}$ reTh, right), a reduction of the response to the standard stimulus occurred rapidly (blue trace), reaching a minimum within the first 50-100 trials, while the response to the oddball stimulus (red trace) showed little adaptation over the course of the train of stimuli. A power law equation $\left(y=a^{\star} x^{b}+c\right)$ provided the best fit to the responses to the standard across trials for all conditions. A high proportion of the adaptation to the standard stimulus was explained by this model (low intensities, $r^{2}=0.923$; medium intensities, $r^{2}=0.952$; high intensities, $r^{2}=0.955 ; p<0.0001$ for all conditions). In contrast, the responses to the deviant stimuli fit poorly to this regression model ( $r^{2}<0.1$ in all conditions). A close inspection of the dynamics of adaptation reveals that intensity level affects inversely the rate of adaptation to the standard stimuli, determined by $b\left(b_{\text {low }}=-0.627 ; b_{\text {med }}=-0.657 ; b_{\text {high }}=\right.$ $-0.577)$, i.e., at higher intensities the adaptation is slower [ $95 \%$ confidence interval $\left(\mathrm{CI}_{95}\right)$ at high intensities, -0.5982 to $-0.5569 ; \mathrm{CI}_{95}$ at medium intensities, -0.6791 to $-0.6346 ; \mathrm{CI}_{95}$ at low intensities, -0.6554 to -0.5993 ]. This suggests that at high sound levels this reduction of the firing rate is not enough to allow an adequate discrimination between the standard and the deviant stimuli (Fig. $6 C$, right), a result that reflects the lower level of adaptation seen at these intensities. It is important to know that the firing rate does not saturate at high intensities (the firing rate keeps increasing gradually from 50 to $80 \mathrm{~dB}$ reTh in a monotonic way) (data not shown), so it is unlikely that the firing rate is putting a ceiling on SSA. 

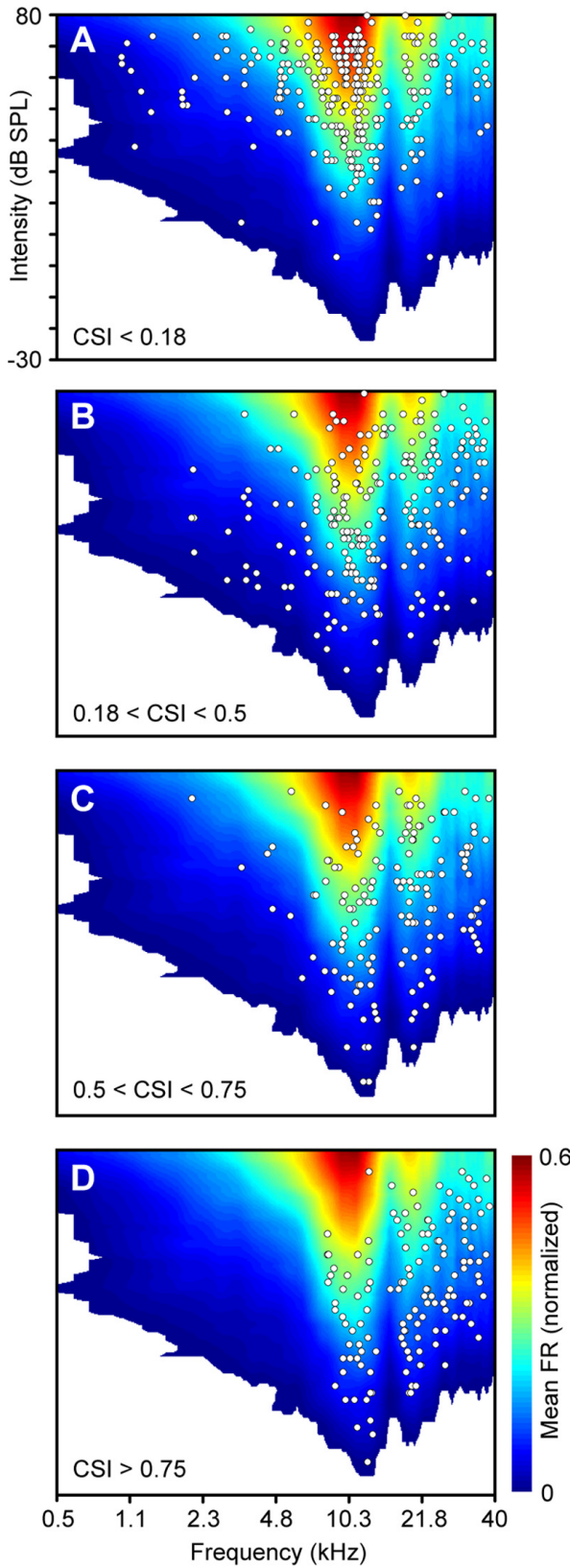

E
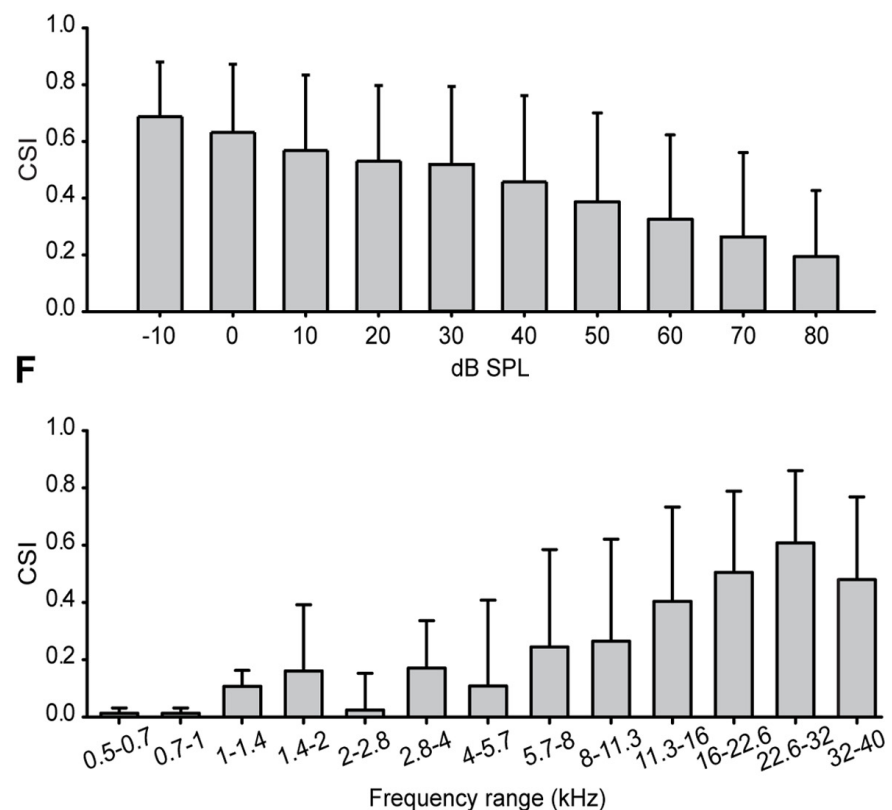

G

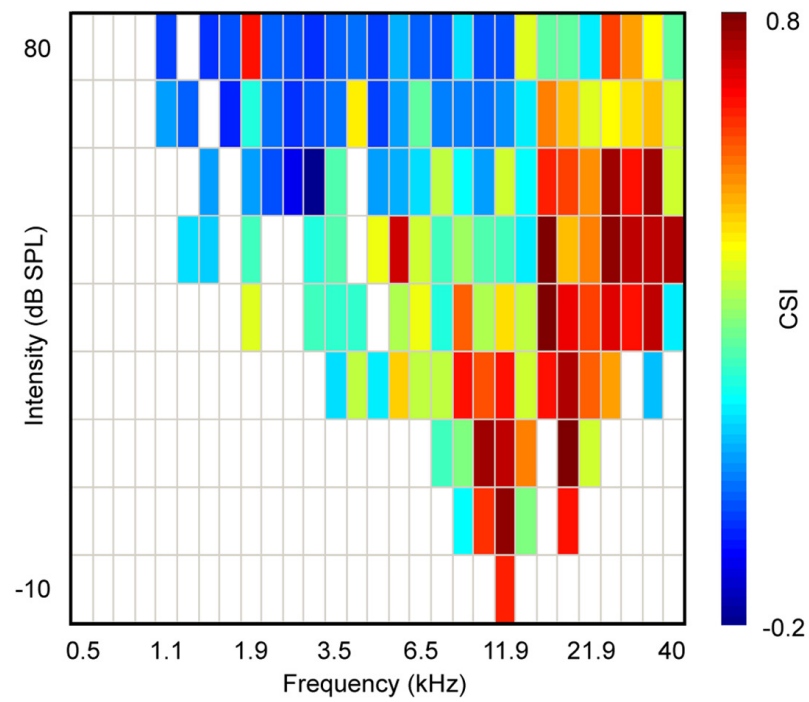

Figure 4. Localization of the frequency pairs in the auditory receptive field sorted by CSI. $A-D$, Cumulative FRA generated by averaging all the FRAs in our sample. The response was normalized to the maximum firing rate of each individual neuron before pooling the FRAs. Each white dot represents the center of a pair of frequencies tested. $A$, Topographic location within the FRA where the lowest CSI levels $(<0.18)$ were found. $\boldsymbol{B}, \boldsymbol{C}$, Topographic location for middle levels of $\mathrm{CSI}(0.18$ to $0.5, \boldsymbol{B} ; 0.5$ to $0.75, \boldsymbol{C})$. D, Topographic location of the pairs of frequencies that evoked the highest CSI levels $(>0.75)$. $\boldsymbol{E}$, Distribution of the levels of CSI sorted by absolute intensities (decibels of SPL). $\boldsymbol{F}$, Distribution of the levels of CSI sorted by absolute frequencies ( 0.5 octaves steps, in kilohertz). $\mathbf{G}$, Raw distribution of the CSI values considering absolute intensity (decibels of SPL, $10 \mathrm{~dB}$ steps) and frequency (in kilohertz, grouped in 0.5 octave steps). Note the different distribution of high CSI values (warm colors) and low CSI values (cold colors). Error bars indicate SD.

\section{SSA as a function of sound frequency}

Next, to check whether the trend of high SSA toward high frequencies is related to the absolute sound frequency (i.e., neurons tuned to high frequencies show stronger SSA) or whether it is actually integrated across the receptive field of the neurons, we established five different groups based on the distance to the best frequency of each neuron: very low frequencies (more than -1 octave of distance from the $\mathrm{BF}$ ), low frequencies (between -1 and -0.2 octaves of distance from $\mathrm{BF}$ ), medium frequencies ( \pm 0.2 octaves of distance from $B F)$, high frequencies (between 0.2 and 1 octaves of distance from $\mathrm{BF}$ ), and very high frequencies $(>1$ octave of distance from the $\mathrm{BF})$. The 0.4 octave window around the $\mathrm{BF}$ was set to integrate similar, but not strictly equal pairs of frequencies. The 2 octave window was set to differentiate 

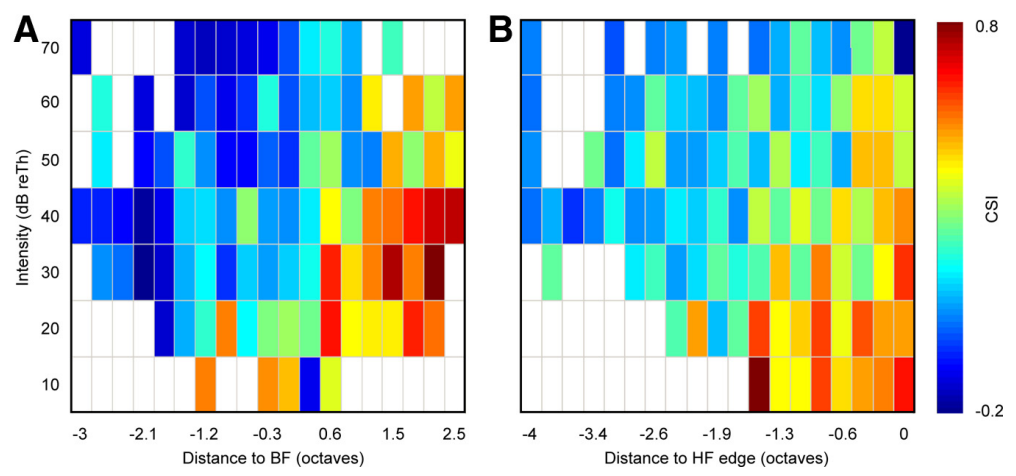

Figure 5. Summary of the distribution of the levels of CSI in the FRA. Mean CSI values found in the neurons as a function of intensity and frequency. $A$, Distribution of the CSI values sorted relatively to the BF and the threshold of the FRA. High CSI values are concentrated at the high-frequency edge. $\boldsymbol{B}$, Distribution of the $\mathrm{CSI}$ values relative to the high-frequency (HF) edge of the FRA for each frequency pair. The values are also standardized to the threshold of each FRA. The distribution of high CSI values along a vertical line demonstrates that there is not a bias due to high-frequency neurons.

the most distant parts of the FRA. Figure 7A shows that CSI values in the high-frequency region are significantly larger than those values in the low-frequency one (Kruskal-Wallis test, $p<0.001$ ). Post hoc comparisons confirmed differences between groups (Dunn's method, $p<0.05$ in all comparisons but those between low and very low, and high and very high frequencies, which were not significant).

Then, we plotted the latency difference based on the five groups established previously. Figure $7 B$ shows that the latency difference tends to be larger for frequencies above BF (KruskalWallis test, $p<0.001)$. Dunn's post hoc analysis confirmed differences between groups (Fig. $7 B$ ). In that case, the latency of both standard and deviant is shorter at the best frequency (Table 2 ) and then increases when the frequencies are more distant to the best frequency. Latencies are slightly larger at the highfrequency region compared to the ones at the low frequency region.

Last, we analyzed the time course of adaptation, establishing three groups relative to the $\mathrm{BF}$ of each neuron: low frequencies (less than -0.2 octaves from BF), best frequency (between -0.2 and 0.2 octaves), and high frequencies $(>0.2$ octaves from BF; Fig. $7 C$ ). As for the intensity analysis described above, a power law equation was the best fit of the response to the standard tone: low frequencies, $r^{2}=0.933$; best frequency, $r^{2}=0.952$; and high frequencies, $r^{2}=0.951$ ( $p<0.0001$ for all conditions). Deviant stimuli responses fit poorly to this regression model $\left(r^{2}<0.1\right.$ in all conditions). These results show that the frequency relative to the $\mathrm{BF}$ affects the dynamics of adaptation by allowing a greater discrimination between the standard and the deviant sounds at high frequencies than at low frequencies. Although the speed of adaptation to the standard stimulus is higher around the $\mathrm{BF}$ $\left(b_{\text {low }}=-0.592 ; b_{\mathrm{BF}}=-0.659, b_{\text {high }}=-0.595 ; \mathrm{CI}_{95}\right.$ around $\mathrm{BF}$, -0.6814 to $-0.6370 ; \mathrm{CI}_{95}$ at low frequencies, -0.6178 to $-0.5666 ; \mathrm{CI}_{95}$ at high frequencies, -0.6167 to -0.5734$)$, the decrement of the response to the standard stimulus is larger at high frequencies than at the lower ones, exhibiting a larger amount of adaptation at this frequency range.

\section{SSA in relation to the temporal pattern of the neuronal response}

An important feature of the neuronal responses is their temporal pattern. Thus, we also studied whether the type of response could be related to the level of SSA. We classified the neuronal responses of our sample in five different groups: ON, LONGLATENCY ON, ON-SUSTAINED, SUSTAINED, and ON-OFF (Rees et al., 1997) (Fig. 8). ON responses showed a robust response confined to the first $40 \mathrm{~ms}$ of the stimulus. The LONG-LATENCY ON response started 50-80 ms after the beginning of the tone. ON-SUSTAINED responses exhibited a clear ON response followed by a SUSTAINED portion, with a lower firing rate than the $\mathrm{ON}$ portion. We defined the ON part of the ONSUSTAINED response as the early portion with a higher firing rate (at least 50\% more than the sustained portion). SUSTAINED responses showed a constant response that lasts $50 \mathrm{~ms}$ or more. To further analyze whether there were differences on SSA between the ON and the SUSTAINED portion of the SUSTAINED response, we defined the ON portion as the first $20 \mathrm{~ms}$ of response. The ON-OFF response had two different latency components in the response, with an ON and OFF portion (after the ending of the stimulus). In our sample, we did not find any pauser or regular-chopper responses, which are typical of the CNIC (Rees et al., 1997).

As reported previously (Hind et al., 1963; Rees et al., 1997), IC neurons can exhibit different patterns of response depending on the region under examination within the FRA. However, at this point, we should emphasize that the majority of the neurons in our sample are from outside the CNIC (Fig. 2). In some cases analyzing the responses of a pair of frequencies, each frequency can present a different pattern of response (i.e., one frequency evokes an ON response and the other one an ON-SUSTAINED response), and also the same frequency can respond differently when the sound is frequent or rare. Thirty-four pairs of frequencies were not analyzed for this reason. Our sample includes 679 pairs of frequencies that showed an ON response, 123 that were ON-SUSTAINED, 16 that were SUSTAINED, 21 that were ONOFF, and 27 that were LONG-LATENCY ON. Sometimes (21 of 87 neurons, $24.14 \%$ ) increasing the sound level caused the response of a neuron to change from an ON response to a SUSTAINED ( 2 of 87 ), ON-OFF (4 of 87 ), or ON-SUSTAINED response (15 of 87 ).

As expected from previous work (Pérez-González et al., 2005; Malmierca et al., 2009), the analysis of the strength of SSA as a function of the response type (Fig. $8 \mathrm{~A}$ ) shows that the ON responses evoke the larger CSI. The median SSA in ON types (CSI, 0.401 ) was significantly different from that in ON-SUSTAINED (CSI, 0.235) and SUSTAINED (CSI, 0.059) types (Kruskal-Wallis ANOVA on ranks, $p<0.001$; post hoc Dunn's method analysis confirmed differences with $p<0.05$ ). The same analysis also showed differences between the ON type and the OFF portion of the ON-OFF response (CSI, 0.153; $p$ values the same for Kruskall-Wallis and Dunn's). Additionally, CSI values of the ON portion in the ON-OFF response are larger than the values of the OFF portion: these data suggest that the $\mathrm{ON}$ portion of the whole neuronal response is more sensitive to SSA.

To further analyze this effect, we examined whether the $\mathrm{ON}$ portion of the ON-SUSTAINED, SUSTAINED, and ON-OFF responses better encoded the SSA (Fig. $8 \mathrm{~B}$ ). In the ONSUSTAINED and the ON-OFF responses, we observed a signifi- 
A

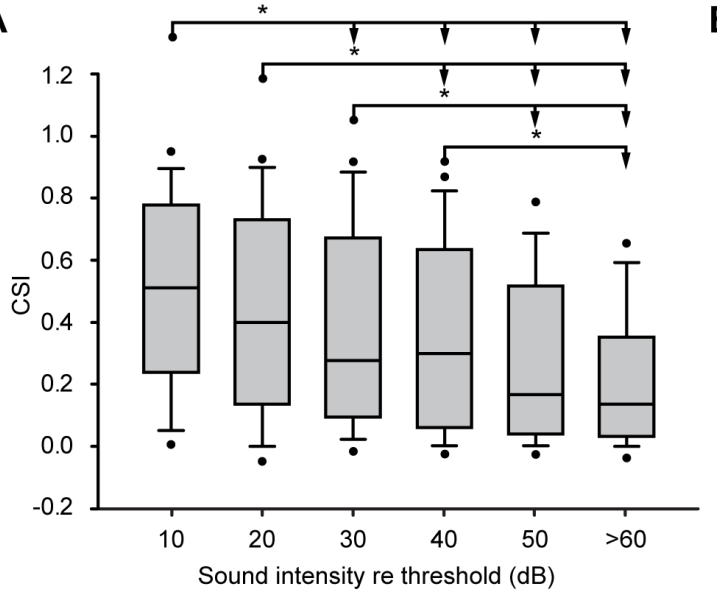

B

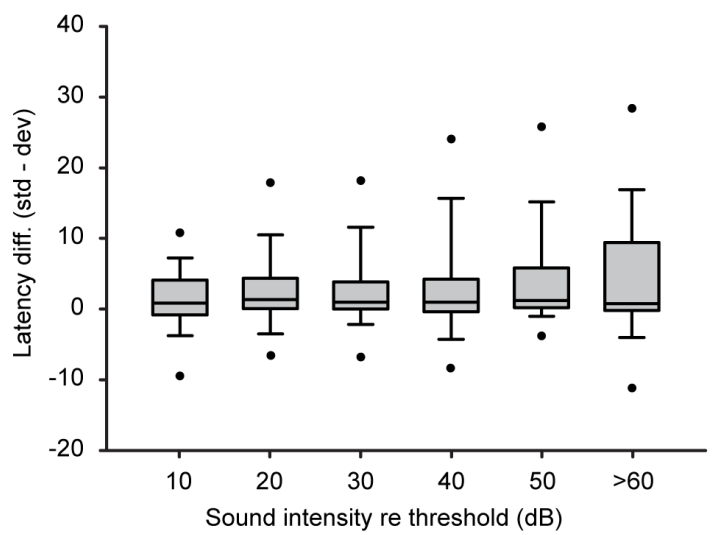

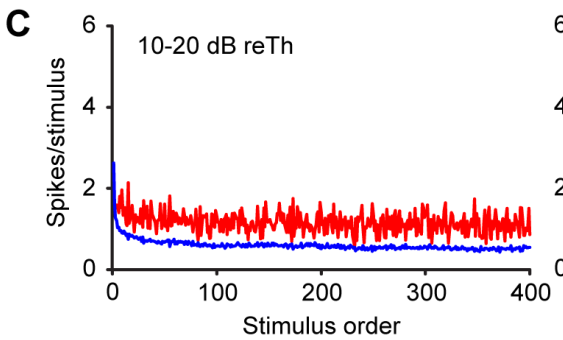
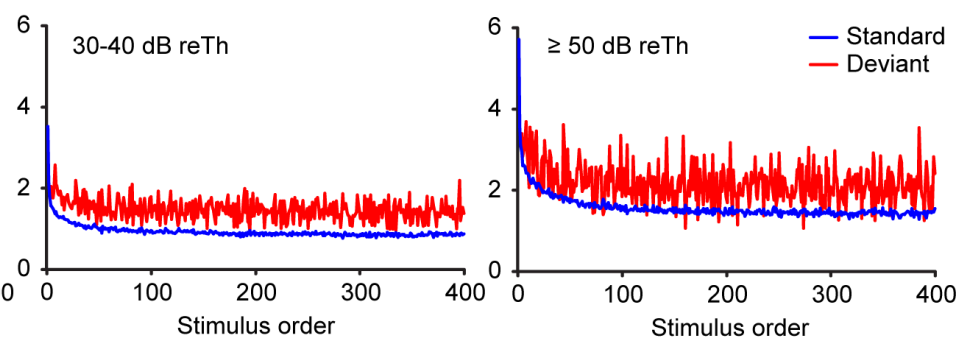

Figure 6. CSI analysis by intensity. $\boldsymbol{A}$, Box plots showing the distribution of the levels of CSI sorted by intensities relative to the neuronal threshold. The asterisks indicate significant differences (Kruskal-Wallis test, $p<0.001$; Dunn's method, $p<0.05$ ). B, Box plots of the latency difference (in milliseconds) between deviants and standards, sorted by intensities relatives to threshold. In both charts $(\boldsymbol{A}$ and $\boldsymbol{B})$, the middle line represents the median value, the box delimits the 25 th and 75 th percentiles, whiskers indicate the 10 th and 90 th percentiles, and the dots indicate the 5 th and 95 th percentiles. $C$, Time course of adaptation sorted by intensities relative to the neuronal threshold. Blue lines show the time course of the standard tones, and red lines show the time course of the deviant tones. Left, Mean time course of the response at low intensities ( $10-20 \mathrm{~dB}$ reTh). Middle, Middle intensities ( $30-40 \mathrm{~dB}$ reTh). Right, High intensities ( $\geq 50 \mathrm{~dB}$ reTh).

Table 1. Median values of the latency of the response (in milliseconds) per intensity

\begin{tabular}{|c|c|c|c|}
\hline \multirow{2}{*}{$\begin{array}{l}\text { Intensity } \\
\text { (dB reTh) }\end{array}$} & \multicolumn{2}{|c|}{ Median first spike latency (ms) } & \multirow{2}{*}{$\begin{array}{l}\text { Latency } \\
\text { difference (ms) }\end{array}$} \\
\hline & Standard & Deviant & \\
\hline 10 & 23.84 & 21.30 & 0.58 \\
\hline 20 & 20.86 & 18.82 & 1.09 \\
\hline 30 & 18.78 & 17.62 & 0.70 \\
\hline 40 & 19.49 & 18.72 & 0.69 \\
\hline 50 & 17.60 & 16.62 & 0.95 \\
\hline$<60$ & 18.63 & 18.18 & 0.53 \\
\hline
\end{tabular}

cant difference in the CSI levels between the ON portion and the SUSTAINED/OFF portion (Wilcoxon signed rank test, $Z=-5.053, p>0.001$; Mann-Whitney rank sum test, $t=344$, $p=0.028$, respectively), whereas in the SUSTAINED response there were no significant differences between the levels founds in the ON and the SUSTAINED portions (Wilcoxon signed rank test, $Z=-1.099, p=0.296)$. As before, these data indicate that a distinct ON portion of the response better encodes SSA.

To evaluate the relationship between the type of response and the latency of the response, we considered the latency difference (Fig. 8C): we observed that the latency difference was significantly larger in the ON-SUSTAINED responses than in the ON and the LONG-LATENCY ON responses (Kruskal-Wallis ANOVA on ranks, $p<0.001$; post hoc Dunn's method analysis, $p<0.05$ in the two cases). This distinction was correlated with a more prominent effect of inhibition over the ON than the SUSTAINED region of the response (Pérez-González et al., 2012), causing the standard response to be reduced or abolished in the ON and LONG-LATENCY ON cases (short latency difference), but being unable to do the same in the ON-SUSTAINED case (longer latency difference).

\section{SSA in relation to spectral properties of the neuronal response}

Last, we studied how the type of FRA is related to the level of SSA. For this purpose we classified the FRAs into six different groups: multipeaked/U-shaped $(n=48), \mathrm{V}$-shaped $(n=20)$, mosaic $(n=12)$, narrow $(n=9)$, closed $(n=3)$, and low tilt $(n=3)$ (Le Beau et al., 2001; Hernández et al., 2005). There were no high-tilt FRAs in our sample. For convenience, multipeaked and $\mathrm{U}$-shaped FRAs were pooled into a single broadly tuned group. Since our data are biased to the cortical regions of the IC, a majority of the neurons are multipeaked (Hernández et al., 2005). To avoid a bias due to low CSI values at high intensities, we computed only the highest CSI value obtained in each neuron. The data demonstrate that the level of SSA for all the neurons was independent of the FRA type and is similar in all the FRAs analyzed (data not shown; Kruskal-Wallis ANOVA on ranks, $p=0.185)$.

To further analyze the variation of the SSA with the shape of the response area, we evaluated the bandwidth of each FRA related to (1) the region of the IC (Fig. 9A) and (2) the level of SSA 
A

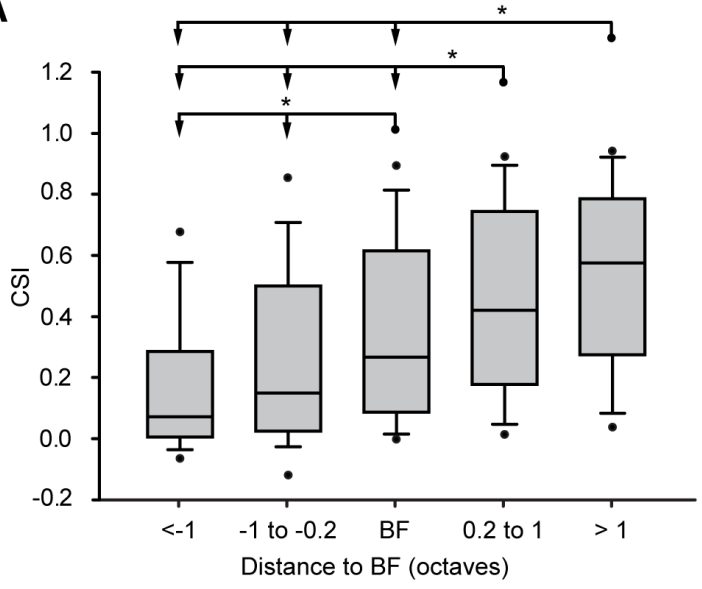

B

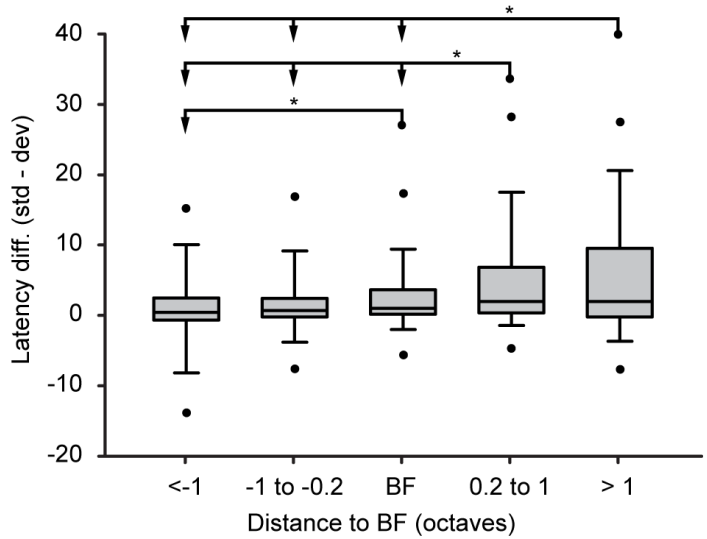

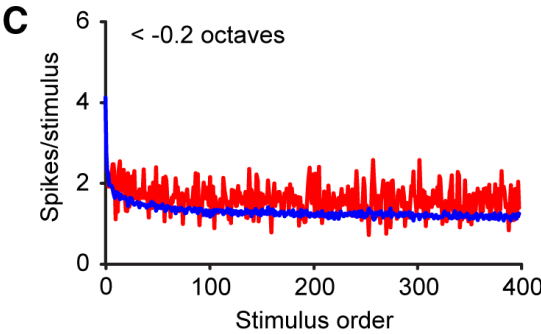
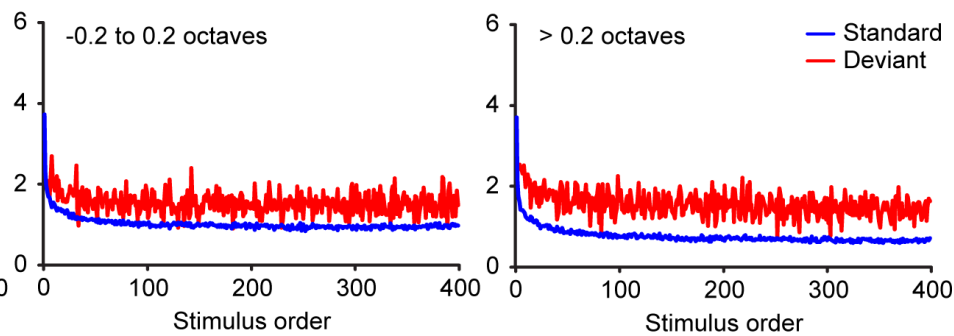

Figure 7. CSI analysis by frequency. $\boldsymbol{A}$, Box plots showing the distribution of the levels of CSI sorted by frequencies relative to the neuronal best frequency (in octaves). The asterisks indicate significant differences (Kruskal-Wallis test, $p<0.001$; Dunn's method, $p<0.05$ ). $B$, Box plots of the latency difference (in milliseconds) sorted by frequencies relative to the neuronal BF. The asterisks indicate significant differences with the Kruskal-Wallis test $(p<0.001)$ and Dunn's method $(p<0.05)$. In both charts $(\boldsymbol{A}$ and $\boldsymbol{B})$, the middle line represents the median value, the box delimits the 25 th and 75 th percentiles, whiskers indicate the 10 th and 90 th percentiles, and the dots indicate the 5 th and 95 th percentiles. $C$, Time course of adaptation sorted by frequencies relative to the neuronal BF. Blue lines show the time course of the standard tones, and red lines show the time course of the deviant tones. Left, Mean time course of the response at low frequencies ( -0.2 octaves lower). Middle, Middle frequencies ( -0.2 to 0.2 octaves). Right, High frequencies ( $\geq 0.2$ octaves).

\begin{tabular}{|c|c|c|c|}
\hline \multirow{2}{*}{$\begin{array}{l}\text { Distance to } \\
\text { BF (octaves) }\end{array}$} & \multicolumn{2}{|c|}{ Median first spike latency (ms) } & \multirow{2}{*}{$\begin{array}{l}\text { Latency } \\
\text { difference (ms) }\end{array}$} \\
\hline & Standard & Deviant & \\
\hline Less than -1 & 19.82 & 18.79 & 0.09 \\
\hline-1 to -0.2 & 18.66 & 18.72 & 0.33 \\
\hline-0.2 to 0.2 & 17.96 & 17.23 & 0.68 \\
\hline 0.2 to 1 & 22.22 & 19.49 & 1.58 \\
\hline$>1$ & 23.01 & 19.69 & 1.57 \\
\hline
\end{tabular}

(B). The CSI groups we used were the same as we had used before (Fig. 4). Despite the clear trend, we did not find significant differences between the groups in the region analysis either at 10 or $40 \mathrm{~dB}$ reTh (Kruskal-Wallis ANOVA on ranks, $10 \mathrm{~dB}$ reTh, $p=$ $0.633 ; 40 \mathrm{~dB}$ reTh, $p=0.073$ ). In the CSI groups we did not find significant differences at $10 \mathrm{~dB}$ reTh (Kruskal-Wallis ANOVA on ranks, $p=0.117)$, but we found differences at $40 \mathrm{~dB}$ reTh $(p<$ 0.024; post hoc Dunn's method confirmed differences between CSIs $<0.18$ and $>0.75$ ). To make the analysis more robust, we pooled the cortical regions (LCIC, RCIC, and DCIC) into a single group, and we did the same with the three adapting groups $(0.18$ to $0.5,0.5$ to 0.75 , and $>0.75)$. $T$ tests confirmed that cortical regions had broader FRA ( $t$ test, $40 \mathrm{~dB}$ reTh, $t=351 ; p=0.014$ ) and that the broader the FRA is, the higher the CSI levels are $(t$ test, $40 \mathrm{~dB}$ reTh, $t=669 ; p=0.005)$.

\section{Discussion}

Our results demonstrate that in the IC, SSA is not constant within the neuronal receptive field, and therefore is not a characteristic property of the neuron. In most cases, a single neuron can exhibit CSI values as high as 1 in some regions of the FRA as well as values close to 0 in others. Our study further demonstrates that higher levels of SSA are biased toward low intensity levels and to the highfrequency edge of the FRA. Furthermore, the type of temporal response pattern observed in each particular region of the FRA is also related to the magnitude of the CSI. Thus, the ON responses exhibit larger SSA values than other response types. We also demonstrated that the neurons with broader receptive fields show more SSA, and most of them are located in the cortical regions of the IC.

\section{Comparison with previous studies and} technical considerations

Previous studies of SSA based on the oddball paradigm considered only a single pair of frequencies or a very restricted area of the neurons' receptive field (Bauerle et al., 2011). In the present study, we show a robust set of data (recording up to 24 pairs of frequencies per neuron) that reveals that SSA sensitivity is not homogeneous, but rather varies across the response area. Thus, a single CSI value cannot be used to completely define the sensitivity for neuronal SSA.

Our study confirms that neurons from the nonlemniscal pathway in IC have higher SSA sensitivity (Malmierca et al., 2009). 

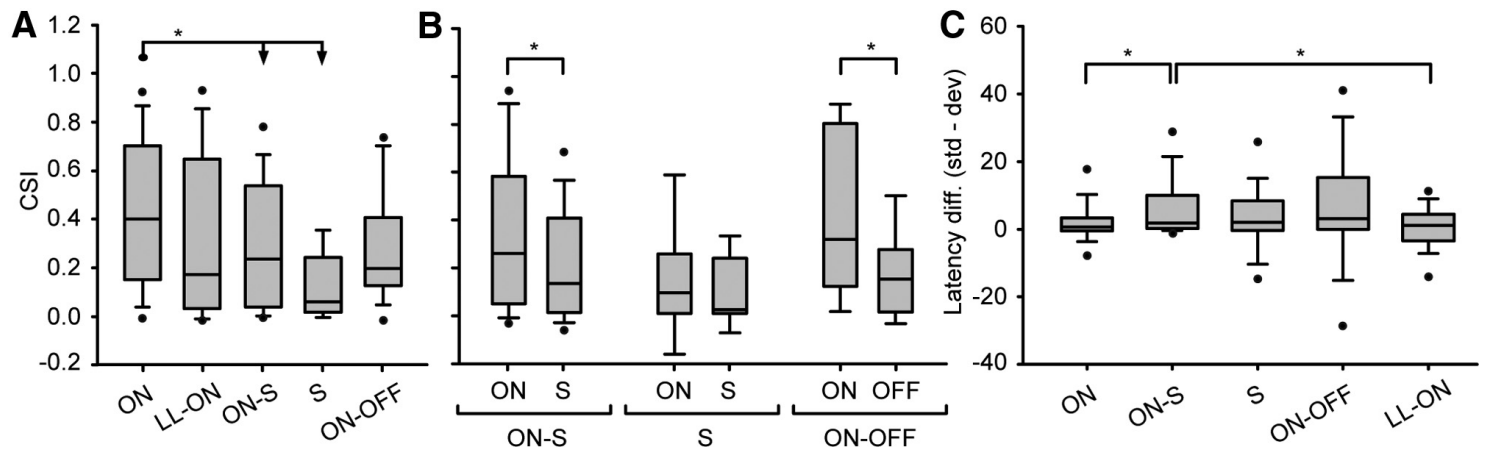

Figure 8. CSI analysis by pattern of response. $A$, Box plot showing the CSI values distributed by the type of response: ON, LONG-LATENCY ON (LL-ON), ON-SUSTAINED (ON-S), SUSTAINED (S), and ON-OFF. The asterisks indicate significant differences (Kruskal-Wallis test, $p<0.001$; Dunn's method, $p<0.05$ ). $B$, Comparison of the CSI values evoked in the ON and SUSTAINED regions of the $0 \mathrm{~N}-\mathrm{S}$ and $\mathrm{S}$ responses, and the $0 \mathrm{~N}$ and $\mathrm{OFF}$ regions in the $0 \mathrm{~N}-0 \mathrm{FF}$ response. The asterisks indicate significant differences (Wilcoxon test, $p<0.001$ and Mann-Whitney test, $p=0.028$, respectively). C, Box plots representing the median latency difference (black line) sorted by the type of response. The asterisks indicate significant differences (Kruskal-Wallis test, $p<0.001 ;$ Dunn's method, $p<$ $0.05)$. In all cases, the middle line represents the median value, the edges of the box delimit the 25 th and 75 th percentiles, whiskers indicate the 10 th and 90 th percentiles, and the dots indicate the 5th and 95th percentiles.

Neurons in the LCIC, DCIC, and RCIC have the largest and less oriented dendritic arbors in the IC (Malmierca et al., 1993, 1995, 2011), allowing neurons to integrate inputs over a broader frequency range. As we show here (Fig. 9A), neurons in the IC cortex possessed broader FRAs. Although the data do not show correlations between the three factors (CSI, bandwidth, and IC subdivisions), it is likely that the higher levels of SSA found in the cortical regions are related to the width of the FRA. Similar relations between adaptation, anatomical subdivisions, and the shape of the FRA have been shown previously in the external cortex of the barn owl (Gutfreund and Knudsen, 2006), a structure homologous to the same region in the rat IC (Knudsen, 1983; Gutfreund and Knudsen, 2006).

Our results support previous studies that reported that SSA is stronger for the ON responses of the neurons both in the IC (Pérez-González et al., 2005; Malmierca et al., 2009; Lumani and Zhang, 2010; Zhao et al., 2011) and the MGB (Antunes et al., 2010; Antunes and Malmierca, 2011). Moreover, our data demonstrate that the ON portion of other response types with a well-defined ON region, like ON-OFF and ON-SUSTAINED responses, show larger SSA as well.

A previous study (Bauerle et al., 2011) concluded that higher levels of SSA were associated with the outer regions of the FRA at high intensity levels. This is partially in agreement with our results. However, we found increased levels of SSA only at the highfrequency domain, regardless of the intensity of the stimulus. Since we used a different animal model and anesthesia as well as different analytical tools and studied different auditory regions, it is difficult to make comparisons and reconcile the discrepancies between these studies.

It remains unknown whether or not the frequency and intensity biases that we demonstrate here have behavioral relevance for rats. Because of the time required to obtain data, we focused on stimulation parameters (frequency contrast, $\Delta f=0.1$; interstimulus interval, $4 \mathrm{~Hz}$ ) that have been proven previously to elicit consistent and large SSA (Malmierca et al., 2009; Antunes et al.,
2010). Further experiments are needed to test whether other conditions that have an effect on SSA (i.e., different frequency separations or repetition rates) would yield comparable results.

\section{SSA is stronger near the neuron's threshold and on the} high-frequency side of the response area

The most important finding of this paper is that low sound intensities and high frequencies evoke stronger SSA than other frequency-level combinations. Because of the adaptation process, the response to the standard stimulus at low intensities disappears gradually after a few repetitions, resulting in a high CSI. During this process, the latency of the response to the standard stimulus increases compared to the latency of the responses to the deviant (Table 1). At high intensities, the response to the standard stimulus is more sustained, and the net result is a low CSI value, because there is no shift in the latency to the standard stimulus due to adaptation. It has been shown that both GABA and glycine control the nonmonotonicity (Faingold et al., 1991), the temporal responses (Le Beau et al., 1996), and the FRA shape of many IC neurons (Le Beau et al., 2001). Although similar mechanisms may be involved in producing SSA, it is unlikely that inhibition alone could explain the differences in CSI values within the intensity domain, because inhibition plays a less prominent role in controlling these factors, i.e., nonmonotonicity, at low intensities 
(Sivaramakrishnan et al., 2004). Indeed, a previous study performed in our laboratory demonstrated that $\mathrm{GABA}_{\mathrm{A}}$ mediated inhibition mainly acts by controlling the gain of the responses, so this type of inhibition plays a minor role in the generation of SSA (Pérez-González et al., 2012). However, since GABA mainly affects the fast component of adaptation (Pérez-González et al., 2012) and the adaptation to the standard is faster at low and middle intensities, it is apparent that multiple mechanisms could be interacting to generate SSA.

Another important feature that we have revealed here is that SSA is significantly stronger on the high-frequency edge of the FRA. Moreover, latency differences at high frequencies show that the latency of the response to the standard tone is larger than that to the deviant stimulus (Fig. $5 C$ ), implying that there is some specific effect on the standard tone in the high-frequency range of responses that is not present in the low-frequency range. Since inhibition precedes excitation in many IC neurons, (Fubara et al., 1996) and GABA inhibition affects SSA (Pérez-González et al., 2012), it is tempting to speculate that the high values of latency difference at high frequencies might relate to an early inhibitory input that holds back the response to the standard sound (Table 2), which in turn would create the differences in the SSA values between the low- and the high-frequency sounds that we observed.

\section{SSA mechanisms and functional significance}

The frequency dependence of SSA may be explained by (1) different inputs to the same neuron, depending mostly on frequency, and the existence of different processes of adaptation associated with these inputs (Ulanovsky et al., 2004); (2) a lateral inhibition network (de la Rocha et al., 2008) based on a broader inhibitory field (Wu et al., 2008); and/or (3) the presence of dual and differential inhibitory effects that will affect SSA differentially, one acting primarily in the low-frequency range, the other at high frequency. In bats, Williams and Fuzessery (2011) showed that there are two non-completely overlapping inhibitory receptive fields that shape FM selectivity. These two inhibitory regions arise from different neurochemical inputs: the low-frequency region is mostly under the influence of GABA, whereas the highfrequency region is under the influence of glycine. Whether or not similar inhibitory mechanisms based on this differential effect of GABA and glycine can account for the frequency/intensity dependence of SSA awaits future studies. Another potential mechanism that could contribute to SSA in the IC is the intrinsic membrane properties of the neuron (Abolafia et al., 2011), but this is unlikely because one would expect individual neurons to show a unique CSI value for each neuron, and here we have demonstrated that SSA depends on the specific frequencies that are tested.

SSA, at least in rat auditory cortex, seems to depend on adaptation with a bandwidth of about one-third of an octave (Taaseh et al., 2011). Since our experiments were performed over a narrow frequency range ( 0.141 octaves), smaller than the suggested width of the adaptation channels estimated by Taaseh et al. (2011), it could be that such adaptation channels are narrower at low intensities. That would explain the differences between low and high intensities, because narrow channels will allow crossfrequency adaptation at low intensities, but not at high intensities, where the channel will be wider than the frequency separations that we used for this experiment.

SSA lies upstream of the generation of MMN (mismatch negativity) (Ulanovsky et al., 2003; Nelken and Ulanovsky, 2007; Taaseh et al., 2011), a late component of auditory ERP (Näätänen et al., 1978). While a previous study showed that SSA does not depend on NMDA receptors, whereas MMN does (Farley et al., 2010), Taaseh et al. (2011) suggested that, at least in auditory cortex, SSA shows true deviance detection, as does MMN. It is likely that biasing SSA toward the low-intensity domain of the FRA, as well as to the highest spectral continuum, might help to sharpen sound discrimination (hyperacuity), as suggested by Bitterman et al. (2008). Future studies should analyze whether IC neurons are also sensitive to the violation of the regularity of the tone sequence caused by the presentation of the deviant stimuli (Jacobsen and Schroger, 2001, 2003), because there is growing and convincing evidence that the human auditory brainstem is able to encode regularities in the auditory stimulation history that serve to detect novel events (Grimm et al., 2011; Slabu et al., 2010, 2012).

In conclusion, our study demonstrates that SSA is not a characteristic property homogeneously distributed within the neuronal receptive field, and further, it suggests that at the population level, SSA is a major property of most nonlemniscal IC neurons well suited to the notion that subcortical neurons exhibiting SSA may contribute upstream to the generation of MMN.

\section{References}

Abolafia JM, Vergara R, Arnold MM, Reig R, Sanchez-Vives MV (2011) Cortical auditory adaptation in the awake rat and the role of potassium currents. Cereb Cortex 21:977-990. CrossRef Medline

Aitkin LM, Kenyon CE, Philpott P (1981) The representation of the auditory and somatosensory systems in the external nucleus of the cat inferior colliculus. J Comp Neurol 196:25-40. CrossRef Medline

Anderson LA, Christianson GB, Linden JF (2009) Stimulus-specific adaptation occurs in the auditory thalamus. J Neurosci 29:7359-7363. CrossRef Medline

Antunes FM, Malmierca MS (2011) Effect of auditory cortex deactivation on stimulus-specific adaptation in the medial geniculate body. J Neurosci 31:17306-17316. CrossRef Medline

Antunes FM, Nelken I, Covey E, Malmierca MS (2010) Stimulus-specific adaptation in the auditory thalamus of the anesthetized rat. PLoS One 5:e14071. CrossRef Medline

Bäuerle P, von der Behrens W, Kössl M, Gaese BH (2011) Stimulus-specific adaptation in the gerbil primary auditory thalamus is the result of a fast frequency-specific habituation and is regulated by the corticofugal system. J Neurosci 31:9708-9722. CrossRef Medline

Bitterman Y, Mukamel R, Malach R, Fried I, Nelken I (2008) Ultra-fine frequency tuning revealed in single neurons of human auditory cortex. Nature 451:197-201. CrossRef Medline

de la Rocha J, Marchetti C, Schiff M, Reyes AD (2008) Linking the response properties of cells in auditory cortex with network architecture: cotuning versus lateral inhibition. J Neurosci 28:9151-9163. CrossRef

Duque D, Malmierca MS, Pérez-González D (2010) Frequency and intensity dependent properties of stimulus-specific adaptation in the inferior colliculus. Paper presented at Ninth International Conference of Neuroethology, Salamanca, Spain, August.

Duque D, Pérez-González D, Malmierca MS (2011) Stimulus-specific adaptation in the inferior colliculus: effects of frequency, intensity and firing rate. Paper presented at Thrity-fourth Research Meeting of the Association for Research in Otolaryngology, Baltimore, MD, February.

Faingold CL, Boersma Anderson CA, Caspary DM (1991) Involvement of GABA in acoustically-evoked inhibition in inferior colliculus neurons. Hear Res 52:201-216. CrossRef Medline

Farley BJ, Quirk MC, Doherty JJ, Christian EP (2010) Stimulus-specific adaptation in auditory cortex is an NMDA-independent process distinct from the sensory novelty encoded by the mismatch negativity. J Neurosci 30:16475-16484. CrossRef Medline

Faure PA, Fremouw T, Casseday JH, Covey E (2003) Temporal masking reveals properties of sound-evoked inhibition in duration-tuned neurons of the inferior colliculus. J Neurosci 23:3052-3065. Medline

Fubara BM, Casseday JH, Covey E, Schwartz-Bloom RD (1996) Distribution of GABAA, GABAB, and glycine receptors in the central auditory 
system of the big brown bat, Eptesicus fuscus. J Comp Neurol 369:83-92. CrossRef Medline

Grimm S, Escera C, Slabu L, Costa-Faidella J (2011) Electrophysiological evidence for the hierarchical organization of auditory change detection in the human brain. Psychophysiology 48:377-384. CrossRef Medline

Gutfreund Y, Knudsen EI (2006) Adaptation in the auditory space map of the barn owl. J Neurophysiol 96:813-825. CrossRef Medline

Hara K, Harris RA (2002) The anesthetic mechanism of urethane: the effects on neurotransmitter-gated ion channels. Anesth Analg 94:313-318, table of contents. CrossRef Medline

Hernández O, Espinosa N, Pérez-González D, Malmierca MS (2005) The inferior colliculus of the rat: a quantitative analysis of monaural frequency response areas. Neuroscience 132:203-217. CrossRef Medline

Hind JE, Goldberg JM, Greenwood DD, Rose JE (1963) Some discharge characteristics of single neurons in the inferior colliculus of the cat. II. Timing of the discharges and observations on binaural stimulation. J Neurophysiol 26:321-341. Medline

Hu B (2003) Functional organization of lemniscal and nonlemniscal auditory thalamus. Exp Brain Res 153:543-549. CrossRef Medline

Hu B, Senatorov V, Mooney D (1994) Lemniscal and non-lemniscal synaptic transmission in rat auditory thalamus. J Physiol 479:217-231. Medline

Jääskeläinen IP, Ahveninen J, Belliveau JW, Raij T, Sams M (2007) Shortterm plasticity in auditory cognition. Trends Neurosci 30:653-661. CrossRef Medline

Jacobsen T, Schroger E (2001) Is there pre-attentive memory-based comparison of pitch? Psychophysiology 38:723-727. CrossRef Medline

Jacobsen T, Schroger E (2003) Measuring duration mismatch negativity. Clin Neurophysiol 114:1133-1143. CrossRef Medline

Knudsen EI (1983) Subdivisions of the inferior colliculus in the barn owl (Tyto alba). J Comp Neurol 218:174-186. CrossRef Medline

Le Beau FE, Rees A, Malmierca MS (1996) Contribution of GABA- and glycine-mediated inhibition to the monaural temporal response properties of neurons in the inferior colliculus. J Neurophysiol 75:902-919. Medline

LeBeau FE, Malmierca MS, Rees A (2001) Iontophoresis in vivo demonstrates a key role for GABA(A) and glycinergic inhibition in shaping frequency response areas in the inferior colliculus of guinea pig. J Neurosci 21:73037312. Medline

Lee CC, Sherman SM (2011) On the classification of pathways in the auditory midbrain, thalamus, and cortex. Hear Res 276:79-87. CrossRef Medline

Loftus WC, Malmierca MS, Bishop DC, Oliver DL (2008) The cytoarchitecture of the inferior colliculus revisited: a common organization of the lateral cortex in rat and cat. Neuroscience 154:196-205. CrossRef Medline

Lumani A, Zhang H (2010) Responses of neurons in the rat's dorsal cortex of the inferior colliculus to monaural tone bursts. Brain Res 1351: 115-129. CrossRef Medline

Malmierca MS (2003) The structure and physiology of the rat auditory system: an overview. Int Rev Neurobiol 56:147-211. CrossRef Medline

Malmierca MS, Blackstad TW, Osen KK, Karagülle T, Molowny RL (1993) The central nucleus of the inferior colliculus in rat: a Golgi and computer reconstruction study of neuronal and laminar structure. J Comp Neurol 333:1-27. CrossRef Medline

Malmierca MS, Seip KL, Osen KK (1995) Morphological classification and identification of neurons in the inferior colliculus: a multivariate analysis. Anat Embryol (Berl) 191:343-350. CrossRef Medline

Malmierca MS, Merchán MA, Henkel CK, Oliver DL (2002) Direct projections from cochlear nuclear complex to auditory thalamus in the rat. J Neurosci 22:10891-10897. Medline

Malmierca MS, Hernández O, Falconi A, Lopez-Poveda EA, Merchán M, Rees A (2003) The commissure of the inferior colliculus shapes frequency response areas in rat: an in vivo study using reversible blockade with microinjection of kynurenic acid. Exp Brain Res 153:522-529. CrossRef Medline
Malmierca MS, Izquierdo MA, Cristaudo S, Hernández O, Pérez-González D Covey E, Oliver DL (2008) A discontinuous tonotopic organization in the inferior colliculus of the rat. J Neurosci 28:4767-4776. CrossRef Medline

Malmierca MS, Cristaudo S, Pérez-González D, Covey E (2009) Stimulusspecific adaptation in the inferior colliculus of the anesthetized rat. J Neurosci 29:5483-5493. CrossRef Medline

Malmierca MS, Blackstad TW, Osen KK (2011) Computer-assisted 3-D reconstructions of Golgi-impregnated neurons in the cortical regions of the inferior colliculus of rat. Hear Res 274:13-26. CrossRef Medline

Merrill EG, Ainsworth A (1972) Glass-coated platinum-plated tungsten microelectrodes. Med Biol Eng 10:662-672. CrossRef Medline

Näätänen R (1992) Attention and brain function. Hillsdale, NJ: Erlbaum.

Näätänen R, Gaillard AW, Mantysalo S (1978) Early selective-attention effect on evoked potential reinterpreted. Acta Psychol (Amst) 42:313-329. CrossRef Medline

Nelken I, Ulanovsky N (2007) Mismatch negativity and stimulus-specific adaptation in animal models. J Psychophysiol 21:214-223.

Paxinos G, Watson C (2005) The rat brain in stereotaxic coordinates. Burlington: Elsevier-Academic.

Pérez-González D, Malmierca MS, Covey E (2005) Novelty detector neurons in the mammalian auditory midbrain. Eur J Neurosci 22:2879-2885. CrossRef Medline

Pérez-González D, Malmierca MS, Moore JM, Hernández O, Covey E (2006) Duration selective neurons in the inferior colliculus of the rat: topographic distribution and relation of duration sensitivity to other response properties. J Neurophysiol 95:823-836. Medline

Pérez-González D, Hernández O, Covey E, Malmierca MS (2012) GABA(A)mediated inhibition modulates stimulus-specific adaptation in the inferior colliculus. PLoS One 7:e34297. CrossRef Medline

Rees A, Sarbaz A, Malmierca MS, Le Beau FE (1997) Regularity of firing of neurons in the inferior colliculus. J Neurophysiol 77:2945-2965. Medline

Sivaramakrishnan S, Sterbing-D'Angelo SJ, Filipovic B, D'Angelo WR, Oliver DL, Kuwada S (2004) GABA(A) synapses shape neuronal responses to sound intensity in the inferior colliculus. J Neurosci 24:5031-5043. CrossRef Medline

Slabu L, Grimm S, Escera C (2012) Novelty detection in the human auditory brainstem. J Neurosci 32:1447-1452. CrossRef Medline

Slabu L, Escera C, Grimm S, Costa-Faidella J (2010) Early change detection in humans as revealed by auditory brainstem and middle-latency evoked potentials. Eur J Neurosci 32:859-865. CrossRef Medline

Taaseh N, Yaron A, Nelken I (2011) Stimulus-specific adaptation and deviance detection in the rat auditory cortex. PLoS One 6:e23369. CrossRef Medline

Ulanovsky N, Las L, Nelken I (2003) Processing of low-probability sounds by cortical neurons. Nat Neurosci 6:391-398. CrossRef Medline

Ulanovsky N, Las L, Farkas D, Nelken I (2004) Multiple time scales of adaptation in auditory cortex neurons. J Neurosci 24:10440-10453. CrossRef Medline

von der Behrens W, Bäuerle P, Kössl M, Gaese BH (2009) Correlating stimulus-specific adaptation of cortical neurons and local field potentials in the awake rat. J Neurosci 29:13837-13849. CrossRef Medline

Williams AJ, Fuzessery ZM (2011) Differential roles of GABAergic and glycinergic input on FM selectivity in the inferior colliculus of the pallid bat. J Neurophysiol 106:2523-2535. CrossRef Medline

Wu GK, Arbuckle R, Liu BH, Tao HW, Zhang LI (2008) Lateral sharpening of cortical frequency tuning by approximately balanced inhibition. Neuron 58:132-143. CrossRef Medline

Yu XJ, Xu XX, He S, He J (2009) Change detection by thalamic reticular neurons. Nat Neurosci 12:1165-1170. CrossRef Medline

Zhao L, Liu Y, Shen L, Feng L, Hong B (2011) Stimulus-specific adaptation and its dynamics in the inferior colliculus of rat. Neuroscience 181:163-174. CrossRef Medline 

Study II: GABAergic modulation of SSA

Duque D, Malmierca MS, Caspary DM (2014)

Modulation of stimulus-specific adaptation by GABAA receptor activation or blockade in the medial geniculate body of the anaesthetized rat.

Journal of Physiology 592:729-743.

doi: 10.1113/jphysiol.2013.261941. 



\title{
Modulation of stimulus-specific adaptation by $\mathrm{GABA}_{\mathrm{A}}$ receptor activation or blockade in the medial geniculate body of the anaesthetized rat
}

\author{
Daniel Duque ${ }^{1,2}$, Manuel S. Malmierca ${ }^{1,3}$ and Donald M. Caspary ${ }^{2}$ \\ ${ }^{1}$ Auditory Neurophysiology Unit, Laboratory for the Neurobiology of Hearing, Institute of Neuroscience of Castilla y León, University of Salamanca, \\ Salamanca, Spain \\ ${ }^{2}$ Department of Pharmacology, Southern Illinois University School of Medicine, Springfield, IL, USA \\ ${ }^{3}$ Department of Cell Biology and Pathology, Faculty of Medicine, University of Salamanca, Salamanca, Spain
}

\section{Key points}

- Neurons in the medial geniculate body (MGB), the auditory thalamus, give stronger responses to rare sounds than to repetitive sounds, a phenomenon referred to as stimulus-specific adaptation (SSA).

- The present study sought to elucidate how the inhibitory thalamic circuitry acting at $\mathrm{GABA}_{\mathrm{A}}$ receptors affects the generation and/or modulation of SSA from recordings of single unit responses from MGB. Microiontophoretic application of GABAergic agonists selectively increased SSA indices, whereas application of antagonists selectively reduced SSA values.

- We found that $\mathrm{GABA}_{\mathrm{A}}$-mediated inhibition did not generate the SSA response but regulated the magnitude of SSA sensitivity in a gain control manner.

- These findings advance our understanding of the role of inhibition in coding deviance detection in the MGB.

\begin{abstract}
Stimulus-specific adaptation (SSA), which describes adaptation to repeated sounds concurrent with the maintenance of responsiveness to uncommon ones, may be an important neuronal mechanism for the detection of and attendance to rare stimuli or for the detection of deviance. It is well known that GABAergic neurotransmission regulates several different response properties in central auditory system neurons and that GABA is the major inhibitory neurotransmitter acting in the medial geniculate body (MGB). The mechanisms underlying SSA are still poorly understood; therefore, the primary aim of the present study was to examine what role, if any, MGB GABAergic circuits play in the generation and/or modulation of SSA. Microiontophoretic activation of $G A B A_{A}$ receptors $\left(G_{A B A_{A}} R s\right)$ with $G A B A$ or with the selective $\mathrm{GABA}_{\mathrm{A}} \mathrm{R}$ agonist gaboxadol significantly increased SSA (computed with the common SSA index, CSI) by decreasing responses to common stimuli while having a lesser effect on responses to novel stimuli. In contrast, $\mathrm{GABA}_{\mathrm{A}} \mathrm{R}$ blockade using gabazine resulted in a significant decrease in SSA. In all cases, decreases in the CSI during gabazine application were accompanied by an increase in firing rate to the stimulus paradigm. The present findings, in conjunction with those of previous studies, suggest that $\mathrm{GABA}_{\mathrm{A}}$-mediated inhibition does not generate the SSA response, but can regulate the level of SSA sensitivity in a gain control manner. The existence of successive hierarchical levels of processing through the auditory system suggests that the GABAergic circuits act to enhance mechanisms to reduce redundant information.
\end{abstract}


(Received 11 July 2013; accepted after revision 6 October 2013; first published online 7 October 2013)

Corresponding author D. M. Caspary: Department of Pharmacology, Southern Illinois University School of Medicine, 801 North Rutledge, Springfield, IL 62702, USA. Email: dcaspary@siumed.edu

\begin{abstract}
Abbreviations MGB, medial geniculate body; SSA, stimulus-specific adaptation; CSI, common SSA index; FRA, frequency response area; GABA, $\gamma$-aminobutyric acid.
\end{abstract}

\section{Introduction}

An optimal response to new acoustic information in the presence of continuous sounds is critical for animal survival. In the central auditory system, many neurons adapt to repeated sounds while maintaining responsiveness to uncommon ones, allowing the detection of rare sounds in an otherwise monotonous auditory scene. This phenomenon is referred to as stimulus-specific adaptation (SSA) (Ulanovsky et al. 2003) and has been found from the inferior colliculus (IC) (for a review see Ayala \& Malmierca, 2013) through to the auditory cortex (AC) (Ulanovsky et al. 2003, 2004; von der Behrens et al. 2009; Taaseh et al. 2011; Yaron et al. 2012). SSA is also present in the anaesthetized (Anderson et al. 2009; Yu et al. 2009; Antunes et al. 2010; Antunes \& Malmierca, 2011) and the unanaesthetized (Richardson et al. 2013a) medial geniculate body (MGB), and has been confirmed as strong and widespread in the non-lemniscal pathway (Malmierca et al. 2009; Antunes et al. 2010; Duque et al. 2012). The mechanisms underlying SSA are poorly understood and the role of the inhibitory circuitry in the generation and/or modulation of SSA in the auditory thalamus remains to be delineated.

The MGB in the auditory thalamus is essential for relaying, processing, filtering and attending to acoustic information. It consists of three main divisions: the ventral (MGV), dorsal (MGD) and medial (MGM). The MGV forms the lemniscal division, and the MGD and MGM are each part of the non-lemniscal pathway, which is related to the analysis of complex features of sound and multisensory integration (for reviews, see Winer, 1985; Hu, 2003; Lee \& Sherman, 2011). Synaptic and extrasynaptic inhibition in the MGB is primarily mediated by GABA acting at both $\mathrm{GABA}_{\mathrm{A}}$ and $\mathrm{GABA}_{\mathrm{B}}$ receptors (Bartlett \& Smith, 1999; Richardson et al. 2011) because MGB lacks glycinergic receptors (Aoki et al. 1988; Friauf et al. 1997). GABAergic interneurons are virtually absent in the rat MGB (only 1\%) (Winer \& Larue, 1996; Bartlett \& Smith, 1999), but the MGB receives significant GABAergic projections from the IC (Winer et al. 1996; Peruzzi et al. 1997; Ito et al. 2011) and the thalamic reticular nucleus (TRN) (Rouiller et al. 1985), the two major sources of GABAergic inhibition to the MGB. The GABAergic inputs to the MGB are known to shape the frequency response areas (FRAs) and adjust thresholds of MGB neurons (Suga et al. 1997; Cotillon-Williams et al. 2008).
As Pérez-González and colleagues (2012) have described a gain control role for the inhibitory circuitry of the IC and Yu et al. (2009) have suggested that the GABA inputs to the MGB shape the novelty response, the present study was designed to shed light on the possible role of $\mathrm{GABA}_{\mathrm{A}}$ receptor $\left(\mathrm{GABA}_{\mathrm{A}} \mathrm{R}\right)$-mediated inhibition in the generation of SSA in the MGB of the rat. Microiontophoresis was used to reversibly block or activate $\mathrm{GABA}_{\mathrm{A}} \mathrm{Rs}$ during oddball paradigm stimulation concurrent with recording from well-isolated single units in the MGB. We recorded before, during and after application of: (i) the $\mathrm{GABA}_{\mathrm{A}} \mathrm{R}$ endogenous agonist GABA; (ii) the subunit-selective $\mathrm{GABA}_{\mathrm{A}} \mathrm{R}$ agonist gaboxadol, and (iii) the $\mathrm{GABA}_{\mathrm{A}} \mathrm{R}$ antagonist gabazine. Our results demonstrate that gabazine increased firing rate and decreased the magnitude of SSA, whereas GABA and gaboxadol produced the opposite effect, such that firing rates decreased and the degree of SSA increased. These results support the suggestion that the GABAergic system in MGB does not shape the SSA response, but exerts a modulator gain control effect.

\section{Methods}

\section{Ethical approval}

All experimental procedures were carried out in accordance with protocols approved by the Laboratory Animal Care and Use Committee of Southern Illinois University School of Medicine (SIU Animal Protocol Number: 41-10-002).

\section{Surgical procedures}

Experiments were performed on 23 4-month-old, male Fischer Brown Norway rats. Rats were initially anaesthetized with I.M. injection $\left(1.4 \mathrm{ml} \mathrm{kg}^{-1}\right)$ of a ketamine-HCl $\left(100 \mathrm{mg} \mathrm{ml}^{-1}\right)$ and xylazine $\left(20 \mathrm{mg} \mathrm{ml}^{-1}\right)$ mixture. Anaesthesia was maintained by I.P. injections of urethane [initially $1.3 \mathrm{ml} \mathrm{kg}^{-1}$, then one-third of the initial amount in booster doses; $750 \mathrm{mg} \mathrm{kg}^{-1}$ (Sigma-Aldrich Corp., St Louis, MO, USA)]. Urethane was chosen as an anaesthetic agent because it acts on multiple neurotransmitter systems rather than simply potentiating the effects of inhibitory systems, and its effects are thought to be less problematic than those generated by barbiturates and/or other anaesthetic agents (Hara \& Harris, 2002). 
Body temperature was maintained at $37 \pm 0.5^{\circ} \mathrm{C}$ by a thermostatically controlled heating blanket. Rats were placed in a stereotaxic frame with a customized jaw bar and head holders inside a double-walled, sound-proofed booth (Industrial Acoustic Co., Inc., New York, NY, USA). Prior to surgery, auditory brainstem responses (ABRs) to click and $4 \mathrm{kHz}, 8 \mathrm{kHz}, 16 \mathrm{kHz}$ and $32 \mathrm{kHz}$ tones $(3 \mathrm{~ms}$ duration, $1 \mathrm{~ms}$ ramp, $20 \mathrm{~s}^{-1}$ rate) were obtained to check that the animal had normal hearing. ABR recordings were obtained as previously described (Wang et al. 2009) using a vertex electrode and subcutaneous electrodes in the nose (reference) and neck (ground). Signals were amplified 500,000 times and averaged over 512 trials with hearing thresholds determined visually. None of the animals used in these experiments showed any signs of hearing loss.

\section{Acoustic stimuli and electrophysiological recording}

A craniotomy was performed to expose the cerebral cortex ( $5.5 \mathrm{~mm}$ from the bregma, $3.5 \mathrm{~mm}$ laterally from the midline) over the centre of the MGB (Paxinos \& Watson, 2007). Extracellular single unit responses were recorded using a six-barrel carbon fibre microelectrode (carbon fibre: $>0.8 \mathrm{M} \Omega$, Carbostar-6; Kation Scientific, Minneapolis, MN, USA). Custom software (ANECS, Ken Hancock; Blue Hills Scientific, Boston, MA, USA) controlled Tucker-Davis Technologies (TDT) System III hardware to generate acoustic signals. The signal was amplified (TDT, ED1), transduced (TDT, EC1) and delivered to the right ear canal using polypropylene tubing. The sound system was calibrated offline into a simulated rat ear (Caspary et al. 2005) using a $\frac{1}{4}$ inch microphone (Bruel \& Kjaer, model 4938). Pure tone intensities in dB SPL (sound pressure level) were accurate to $\pm 2 \mathrm{~dB}$ for frequencies up to $45 \mathrm{kHz}$ (Caspary et al. 2005). Search stimuli were $70-80 \mathrm{~dB}$ broadband noise pips. Spike output from the carbon fibre was led to a single channel of a 16-channel unity-gain headstage tethered to a preamplifier [ $2 \times$ gain, $0.15 \mathrm{kHz}$ (high pass), $8 \mathrm{kHz}$ (low pass); Plexon, Inc., Dallas, TX, USA]. Spikes were digitized and visualized using Sort Client, with action potentials/spikes sorted using amplitude threshold and saved as timestamps (Plexon, Inc.).

\section{Stimulus presentation paradigms}

Upon isolating a unit, the approximate frequency response was manually determined by presenting tone-bursts ( $100 \mathrm{~ms}$ duration, $5 \mathrm{~ms}$ rise/fall time, four bursts/s rate). Automated FRAs were then obtained using random combinations of frequencies and intensities evoking a response resulting in a mapped neuronal receptive field. Pure tones were presented using an automated procedure with five stimulus repetitions at each frequency $(0.5-40 \mathrm{kHz}$, in $20-30$ logarithmic steps) and intensity (10 dB SPL intensity steps, 0-80 dB SPL) point. Higher-resolution response maps were used to more accurately determine characteristic frequency (CF) as needed. A collection window was set to count the spike number during the response of the neuron (typically $100 \mathrm{~ms}$ in duration). Minimum thresholds and best frequency $(\mathrm{BF})$ (i.e. the frequency that evoked a response with the lowest intensity) responses were derived from these maps.

The oddball paradigm was used to evaluate SSA. The calculation of the FRA allowed selection of frequency pairs $\left(f_{1}\right.$ and $\left.f_{2}\right)$ that elicited similar firing rates at the same stimulus level. Each frequency in the pair was always presented at the same sound level. Stimuli presented in an oddball paradigm were similar to those used to record mismatch negativity responses in human (Näätänen, 1992) and SSA responses in animal (Ulanovsky et al. 2003, 2004; Malmierca et al. 2009; Antunes et al. 2010; Duque et al. 2012; Ayala et al. 2013; Richardson et al. 2013a) studies. Briefly, 300 stimuli containing both frequencies were presented in a probabilistic manner: one frequency $\left(f_{1}\right)$ presented as a standard sound ( $90 \%$ of occurrence) was interspersed randomly with a second deviant $(10 \%$ of occurrence) stimulus frequency $\left(f_{2}\right)$. After recording responses, the relative probabilities of the two stimuli were reversed. Dot raster plots were used to visualize responses obtained to the oddball paradigm by plotting individual spikes (each dot is a spike: red dots indicate responses to the deviant; blue dots indicate responses to the standard). Presentations were marked along the vertical axis. As the pairs of frequencies were chosen close to the threshold (where the FRA is narrower), the frequency contrast was set at $\Delta \mathrm{f} \approx 0.10$, where $\Delta \mathrm{f}=\left(f_{2}-f_{1}\right) /\left(f_{2} \times f_{1}\right)^{1 / 2}$ (Ulanovsky et al. 2003, 2004; Malmierca et al. 2009). The average stimulus intensity was $17.26 \pm 11.97 \mathrm{~dB}$ above the CF threshold. Stimuli were presented at a rate of four per second, conditions previously shown to evoke strong SSA in the MGB (Antunes et al. 2010; Antunes \& Malmierca, 2011).

SSA responses were quantified by computing the common SSA index (CSI) (Ulanovsky et al. 2003), defined as CSI $=\left[\mathrm{d}\left(f_{1}\right)+\mathrm{d}\left(f_{2}\right)-\mathrm{s}\left(f_{1}\right)-\mathrm{s}\left(f_{2}\right)\right] /\left[\mathrm{d}\left(f_{1}\right)+\right.$ $\left.\mathrm{d}\left(f_{2}\right)+\mathrm{s}\left(f_{1}\right)+\mathrm{s}\left(f_{2}\right)\right]$, where $\mathrm{d}(f)$ and $\mathrm{s}(f)$ are responses to each frequency $f_{1}$ or $f_{2}$ according to whether they represented a deviant (d) or standard (s) stimulus. CSI reflects the extent to which the response to the standard was suppressed. CSI values range between -1 and +1 ; more positive values reflect a greater response to the deviant stimulus. We used the CSI value of 0.18 , defined by Antunes et al. (2010), as the threshold for significant SSA. This cut-off value was established by choosing the most negative CSI value in the dataset $(-0.18)$ to represent the most extreme variance due to random fluctuations in spike counts. For consistency and to enable comparisons, we applied the same value as 
used previously. To minimize the effects of spontaneous activity in the analysis, collection time windows were chosen individually for each unit. The default time window embraced the whole stimulus $(0-100 \mathrm{~ms})$ for low spontaneous activity responses (e.g. Fig. $2 A$ ). For cases of high spontaneous activity (e.g. Fig. $2 B$ ), time windows were set based on the shape of the peristimulus time histogram (PSTH) (i.e. by focusing or narrowing the window around the peak of the response. SSA was also quantified by calculating the frequency-specific index $\operatorname{SI}\left(f_{i}\right)$, where $\mathrm{i}=1$ or 2 , defined for each frequency $f_{i}$ as $\mathrm{SI}\left(f_{i}\right)=\left[\mathrm{d}\left(f_{i}\right)-\mathrm{s}\left(f_{i}\right)\right] /\left[\mathrm{d}\left(f_{i}\right)+\mathrm{s}\left(f_{i}\right)\right]$, where $\mathrm{d}\left(f_{i}\right)$ and $\mathrm{s}\left(f_{i}\right)$ are responses to frequency $f_{i}$ when it is deviant or standard, respectively.

\section{lontophoresis and pharmacology}

When the MGB was located, iontophoretic studies were performed using the six-barrel Carbostar multibarrel electrode. A current-balancing barrel was filled with $2 \mathrm{M}$ potassium acetate and the remaining barrels were filled with drugs purchased from Sigma-Aldrich Corp. These included the $\mathrm{GABA}_{\mathrm{A}} \mathrm{R}$ endogenous agonist $\gamma$-aminobutyric acid (GABA; $500 \mathrm{~mm}, \mathrm{pH} 4.0$ ), the $\mathrm{GABA}_{\mathrm{A}} \mathrm{R}$ subunit-selective superagonist gaboxadol $(10 \mathrm{mM})$ and the $\mathrm{GABA}_{\mathrm{A}} \mathrm{R}$ antagonist gabazine $(10 \mathrm{~mm})$. Iontophoretic current was supplied by a multi-channel iontophoresis system (BH-2 Neuro-Phore System; Harvard Apparatus/Medical Systems, Inc., Holliston, MA, USA) through a silver chloride wire and was generally kept at $0-100 \mathrm{nA}$ to avoid excessive diffusion (Foeller et al. 2001). Candy et al. (1974) have shown that certain small molecules, iontophoretically delivered agents, which are not rapidly removed, can diffuse up to $600 \mu \mathrm{m}$. In the rat MGB, this range would cover most of the extent of the dendritic arbours (MGV: $\sim 280 \mu \mathrm{m}$; MGD: $\sim 400 \mu \mathrm{m}$; MGM: $750 \mu \mathrm{m}$ ) (Clerici et al. 1990; Bartlett \& Smith, 1999; Smith et al. 2006). However, we cannot exclude the possibility that agents used here may have affected GABA receptors located at more distant dendritic branches. Recording and iontophoretic procedures were similar to those described elsewhere (e.g. Backoff et al. 1999; Caspary et al. 2002; Pérez-González et al. 2012). A full return to baseline/pre-drug level was set, regardless of the time, before additional agents were applied. For each unit studied, the dose and time of application were varied $(0-100 \mathrm{nA}, 1-20 \mathrm{~min})$ with the aim of achieving a steady state level of drug action.

\section{Data analysis}

Statistical tests were performed using the multiple non-parametric Friedman signed rank test to test differences between distribution medians of varying conditions. Post hoc comparisons were performed following Dunn's method. Statistical tests were considered significant when $P<0.05$. The S.D. for the CSI and the firing rate of each individual neuron were calculated using bootstrapping (1000 repetitions). The limits of 95\% confidence intervals (CIs) were calculated using the 2.5 and 97.5 percentiles of the CSI bootstrap distribution obtained for each neuron; the $5 \%$ confidence level was used to determine statistically significant differences in the CSI and firing rate values between conditions. Analyses and figures were executed using Sigmaplot Version 11 (Systat Software, Inc., Chicago, IL, USA) and Matlab (MathWorks, Inc., Natick, MA, USA).

\section{Histological verification}

At the end of each experiment, the animal was perfused with buffered saline followed by $4 \%$ paraformaldehyde and decapitated. The brain was removed and placed in $20 \%$ sucrose overnight. The brain was blocked and $50 \mu \mathrm{m}$ coronal sections were stained with fast thionin. The depth for recording each unit was carefully recorded. As the track left by the Carbostar electrode was readily visible, the need for lesion or dye injection was obviated. Tracks were localized using a rat brain atlas (Paxinos \& Watson, 2007) and previous MGB studies (Bartlett \& Smith, 1999; Antunes \& Malmierca, 2011).

\section{Results}

To study the impact of GABAergic inhibition on SSA sensitivity, we recorded responses to an oddball stimulus paradigm from 52 well-isolated single units throughout the MGB, before, during and after application of gabazine, GABA and gaboxadol. Generally, microiontophoretic application of gabazine increased firing rates and reduced SSA levels, whereas the application of GABA and gaboxadol produced an opposite effect. The detailed effects that $\mathrm{GABA}_{\mathrm{A}} \mathrm{R}$-related agents produce on firing rate, SSA indices, dynamics of adaptation and latency were evaluated separately.

\section{SSA in the MGB}

As expected from previous reports (Antunes et al. 2010), a majority of neurons sampled showed significant SSA [42 of 52 neurons $(81 \%)$, CSI $>0.18$ ] (i.e. they responded preferentially to a deviant tone compared with the commonly occurring standard). Across the population, the full range of CSI values $(-0.001$ to 0.897 ; mean \pm S.D. $0.443 \pm 0.249$; $n=52$ ) was observed (Fig. $1 A$ ). To test the possibility of a differential drug effect over the two frequencies analysed, we checked whether there was any preference for the deviant stimulus according to whether the deviant was presented as $f_{1}$ or $f_{2}$. The analysis of SI values 
confirmed that the majority of values were positive and located in the upper right quadrant $(n=104$; mean \pm S.D. $0.403 \pm 0.325$; one-sample $t$ test, $P \leq 0.001$ ) (Fig. $1 B$ ). The Mann-Whitney rank sum test confirmed there were no differences in the population between $\mathrm{SI}_{1}$ and $\mathrm{SI}_{2}$ values $(P=0.241)$.

Recording sites were localized from histological sections according to the track marks through the MGB; when possible, a neuron was assigned a position based on known depth in one of the three main MGB divisions. Nine tracks in eight rats were well localized. In five rats, in which 11 units were recorded, tracks were assigned to the MGD and MGM. All neurons from the MGD and
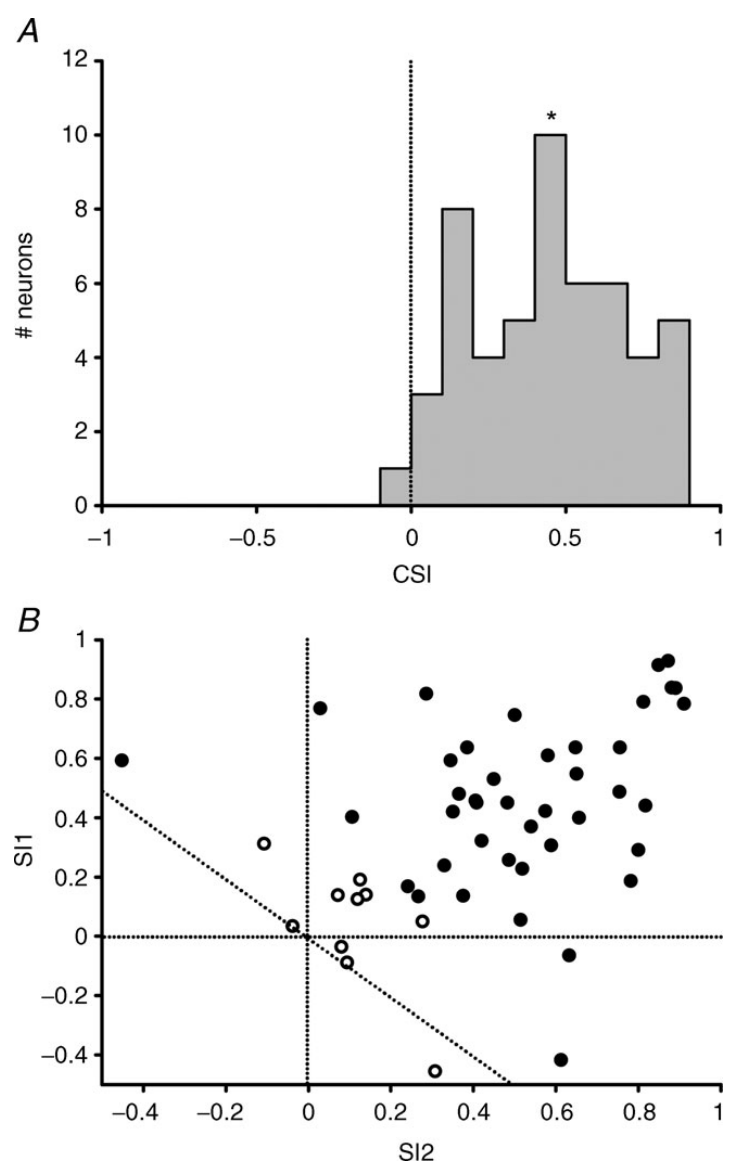

Figure 1. Population data from medial geniculate body (MGB) recordings $(n=52)$ under control conditions

$A$, histogram of common stimulus-specific adaptation index (CSI) values for the MGB population. *, median CSI value. $B$, scatterplot of frequency-specific index $\left(\mathrm{SI}_{1} \mathrm{vs}\right.$. $\left.\mathrm{SI}_{2}\right)$ values for the MGB population. Data lie generally above the diagonal of equal values (black dotted line). Filled dots show neurons with a CSI value higher than the cut-off (CSI > 0.18); empty dots show neurons with a CSI lower than the cut-off $(\mathrm{CSI}<0.18)$.
MGM showed significant SSA. In the other three rats, tracks were localized in the MGD and MGV and yielded six units. Of these, three units exhibited significant SSA and the remaining three lacked SSA. Thus, only 17 of the $52(33 \%)$ recorded units could be accurately localized, but these data support previous findings that SSA is biased towards the non-lemniscal regions of the MGB, where SSA is strong.

\section{Effects of gabazine and GABA on firing rate and SSA}

The application of gabazine and GABA resulted in profound changes in firing rate and SSA in most MGB neurons. Figure 2 illustrates two examples from individual neurons with corresponding FRAs. The two black dots in the FRAs indicate the frequencies used for the oddball paradigm. Dot raster plots were obtained for four conditions: (i) control; (ii) during gabazine application; (iii) during GABA application, and (iv) during recovery following drug application. The corresponding mean PSTHs for both oddball paradigms are shown below the dot raster plots. The PSTHs of adapting frequency pairs exhibit greater responses to deviant sounds (red line), whereas non-adapting pairs have similar responses to both conditions (red and blue lines at the same level). Figure $2 \mathrm{~A}$

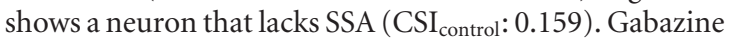
application produced a $96 \%$ increase in firing rate (Fig. 2A, PSTH second column) while slightly lowering

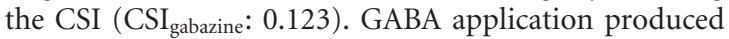
the opposite effect, decreasing firing rate $(42 \%$ decrease in the control response) (Fig. $2 \mathrm{~A}$, PSTH third column) while significantly increasing the CSI $\left(\mathrm{CSI}_{\mathrm{GABA}}\right.$ : 0.412). Another neuron exhibited a high level of SSA (CSI control: $_{\text {: }}$ : $0.528)$, with the application of gabazine resulting in an $80 \%$ increase in firing rate (Fig. $2 B$, PSTH second column) while significantly decreasing CSI (CSI gabazine: $0.375)$. Conversely, GABA reduced the discharge rate by $65 \%$ (Fig. 2B, PSTH third column) while significantly increasing the CSI $\left(\mathrm{CSI}_{\mathrm{GABA}}\right.$ : 0.815). For both neurons cited in Fig. 2, GABA application appeared to suppress the standard relative to the deviant (see also Fig. $3 B$ ).

Fifty-two neurons recorded from MGB were evaluated for drug responses to establish whether: (i) gabazine application generated a significant increase in firing rate $(n=40)$; (ii) GABA application significantly reduced firing rate $(n=42)$, or (iii) both. Although the increase or decrease in the firing rate response between $f_{1}$ and $f_{2}$ was correlated (Spearman's rank order correlation, $P<0.05$ for both standard and deviant responses), the drug effects on both frequencies were analysed separately because a change in firing rate response for a frequency does not necessarily imply the change in the other frequency (52 neurons, 104 frequencies). Conventional responses to the application of $\mathrm{GABA}_{\mathrm{A}} \mathrm{R}$ agonists or antagonists indicated that responses to the standard tended to be 
more affected by $\mathrm{GABA}_{\mathrm{A}} \mathrm{R}$ manipulation than responses to the deviant. This was the case for gabazine (response increase, standard: $87.73 \%$; deviant: $41.66 \%$; paired $t$ test, $Z=-3.036, P=0.002)$, and was especially evident in
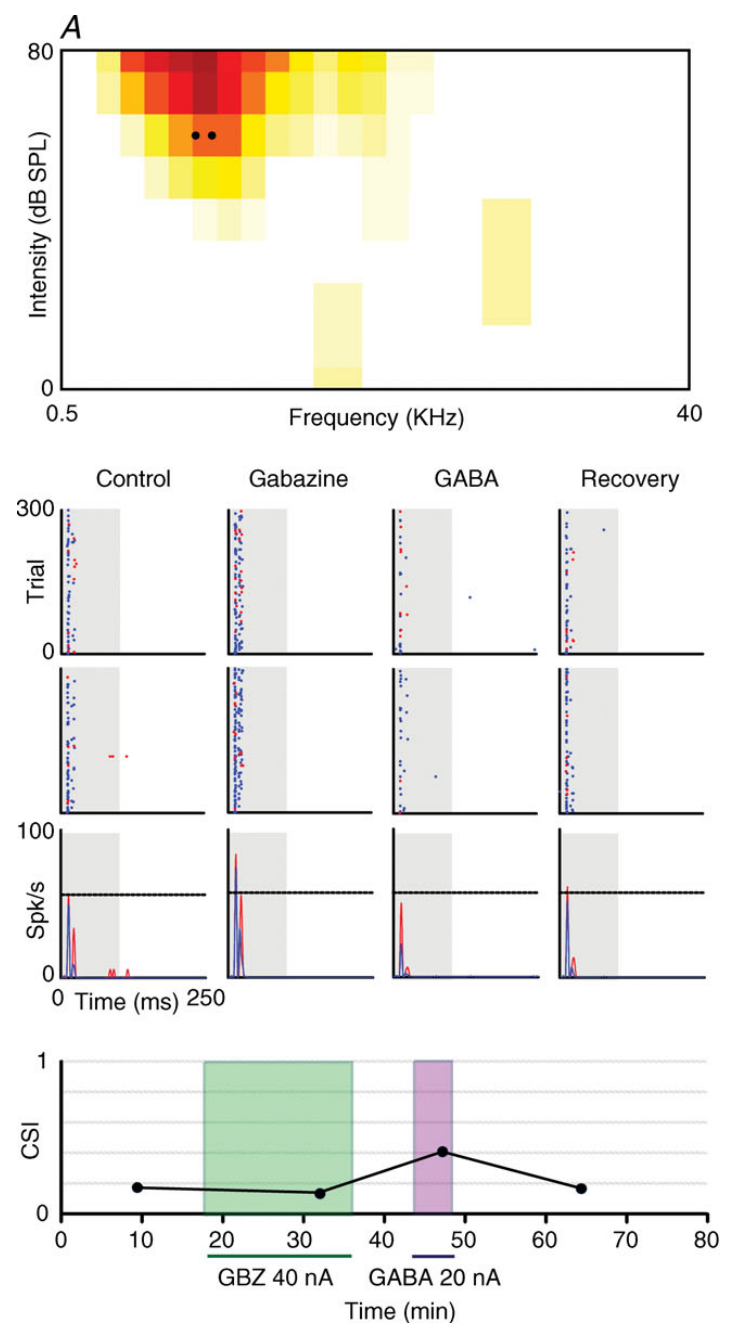

the responses to GABA application in Fig. $3 B$ (response decrease, standard: $72.09 \%$; deviant: $54.10 \%$; paired $t$ test, $Z=-4.085, P<0.001)$. The differential effect of GABA application on the standard, relative to the deviant, is
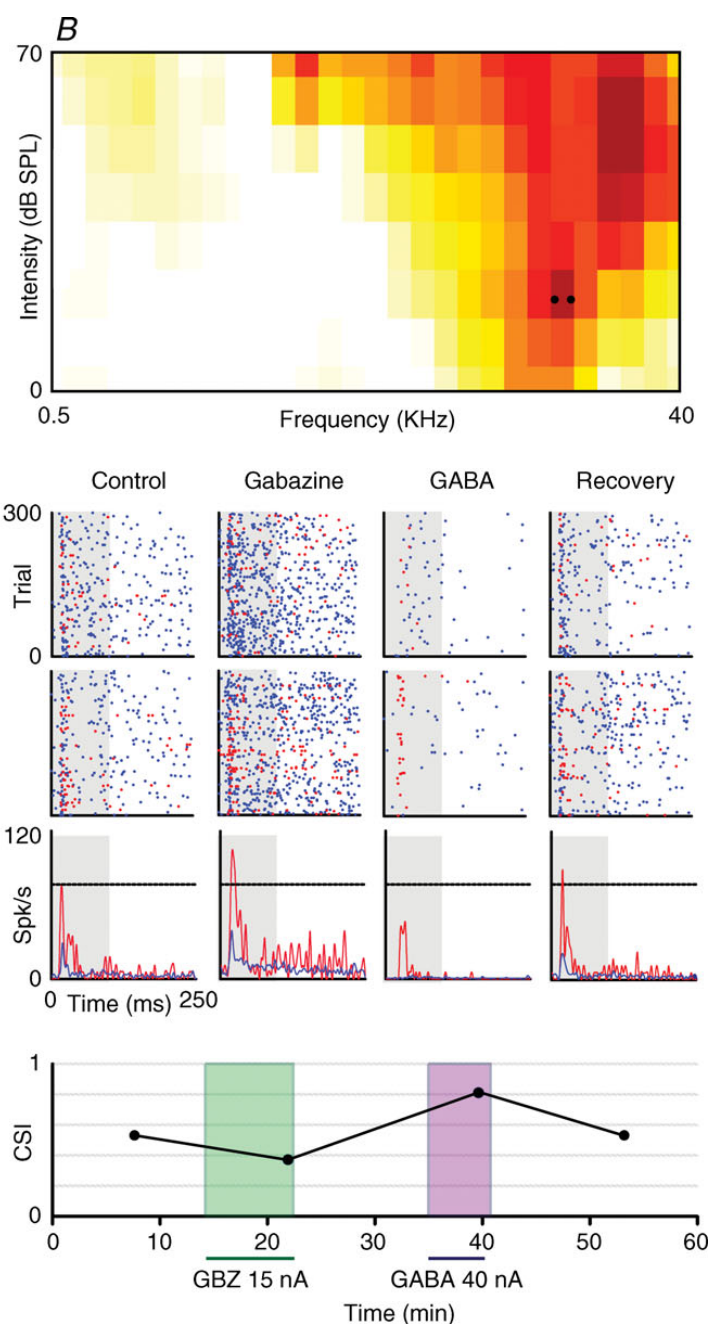

Figure 2. Examples of single unit responses in the medial geniculate body (MGB) before, during and after the application of gabazine and GABA

$A$, frequency response area (FRA) of a neuron in the MGB that did not show a high common stimulus-specific adaptation (SSA) index (CSI) value in the control condition. B, FRA of a neuron in the MGB that showed a high CSI value in the control condition. Black dots over the FRAs represent the pair of frequencies selected for analysing SSA. Below the FRAs, dot raster plots refer to each of the four conditions [control, gabazine (GBZ), GABA and recovery] in the first row $\left(f_{1} / f_{2}\right.$ as standard/deviant) and the reverse condition ( $f_{2} / f_{1}$ as standard/deviant) in the second row. Stimulus presentations are accumulated in the temporal domain in the vertical axis. Blue dots represent spikes evoked by the standard stimulus ( $90 \%$ probability); red dots represent those evoked by the deviant stimulus (10\% probability). The time between trials ( $250 \mathrm{~ms}$; $x$-axis) corresponds to the stimulus repetition rate $(4 \mathrm{~Hz}$; with $75 \mathrm{~ms}$ stimulus duration). The shadow backgrounds in the dot rasters indicate the duration of the stimulus. Below the dot rasters (third row), peristimulus time histograms (PSTHs) show averaged responses for both frequencies when deviant (red) or standard (blue). The dashed horizontal line in the PSTH shows the deviant peak control response. Lower graphs show the evolution of CSI values during the application of the different drugs. In both examples, neurons in the recovery condition returned to the CSI level of the control/pre-drug condition. 
A

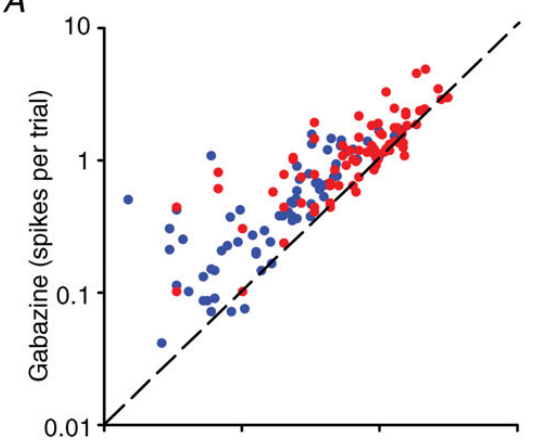

\section{B}

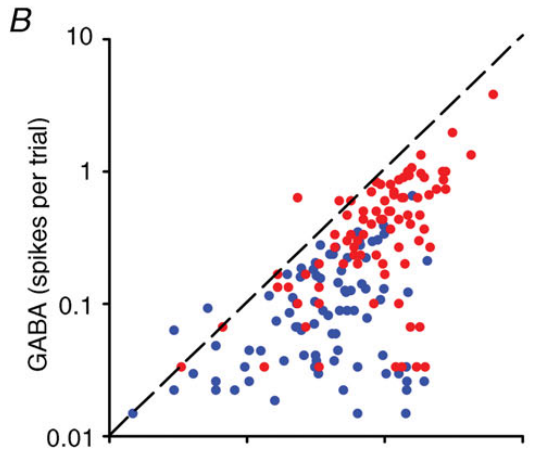

C

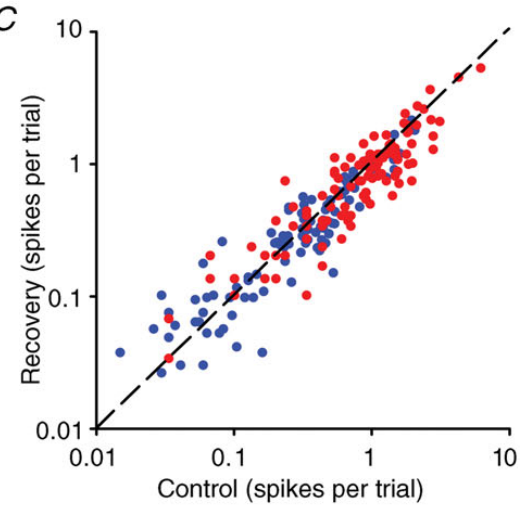

$D$

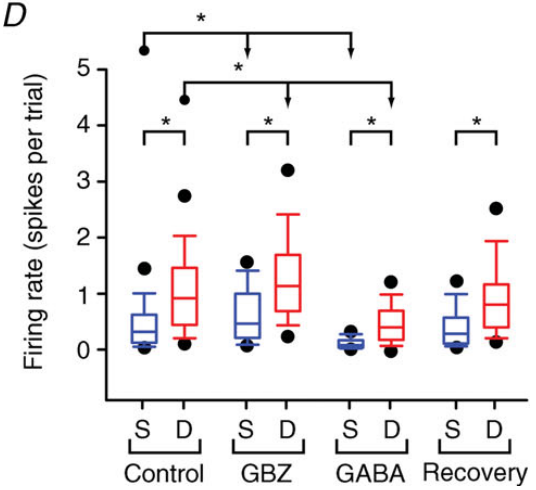

shown in Fig. $3 B$ by the cluster of blue dots (standard) below the diagonal line, relative to the number of red dots (deviant) below the diagonal line. In a few cases $(n=9)$ we noted paradoxical effects that depended on the frequency used for stimulation $\left(f_{1}\right.$ or $\left.f_{2}\right)$ or the nature of stimuli (standard or deviant), whereby gabazine slightly decreased the response to one frequency (five dots under the diagonal dashed line, Fig. $3 A$ ) or GABA increased it (four dots over the diagonal dashed line, Fig. 3B). These paradoxical effects may occur through small changes in the membrane potential that drive the MGB neuron into burst or tonic mode. In order to evaluate the effects of gabazine and GABA, we used the bootstrap method over 1000 randomizations to estimate the $95 \%$ CI of the firing rate response for each neuron in the control condition (data not shown). Then, the firing rates obtained for the gabazine, GABA and recovery conditions for each neuron were compared with the $95 \% \mathrm{CI}$ firing rate generated for the control condition. We accepted: (i) the finding for gabazine if it presented a firing rate higher than the $95 \%$ $\mathrm{CI}$ in the control condition; (ii) the finding for GABA if it showed a firing rate lower than the $95 \% \mathrm{CI}$ in the control condition, and (iii) the finding for the recovery condition if the firing rate response in that condition lay within the $95 \% \mathrm{CI}$ of the control condition (Fig. 3C). (Note that charts $A-C$ in Fig. 3 are logarithmic. We used this rather than a linear representation to stretch the data that would have been clustered near the origin.) Therefore, at the population level, the firing rates evoked by both the standard and the deviant stimuli were significantly higher when gabazine was applied (Fig. $3 D, n=80$ ) and lower when GABA was applied (Fig. $3 D, n=84$ ). Findings in the control condition $(n=104)$ were also compared with those in the recovery condition $(n=88)$. A summary of firing rates obtained in each condition is shown in Table 1. Friedman's repeated-measures ANOVA on ranks found that median values for the standard stimuli differed

Figure 3. Effects of gabazine and GABA on firing rate in the medial geniculate body (MGB) population

$A-C$, scatterplots of the responses (spikes/stimulus) of all neurons to the deviant (red dots) and standard (blue dots) stimuli in the control vs. gabazine $(A), G A B A(B)$ and recovery $(C)$ conditions. Dots represent one of each frequency analysed separately in every pair of stimuli recorded with the oddball paradigm (52 neurons; 104 frequencies). $A$, gabazine differentially increases the response rate to common/standard stimuli and has a smaller effect on the response rate to novel stimuli. $B$, GABA decreases the response in almost all neurons, having a greater effect on responses to common than to novel stimuli. $C$, the recovery condition shows a return to the control/pre-drug condition. $D$, distribution of the mean response magnitude changes across the population of neurons for the control, gabazine (GBZ), GABA and recovery conditions, for deviant (red) and standard (blue) stimuli. *, significant differences (Friedman's test, $P<0.01)$. 
Table 1. Firing rates of the standard and deviant stimuli at different conditions (spikes/stimulus)

\begin{tabular}{lccccccc}
\hline & \multicolumn{3}{c}{ Standard stimuli } & & \multicolumn{3}{c}{ Deviant stimuli } \\
\cline { 2 - 3 } \cline { 6 - 7 } Condition (drug) & Median & $25 \%$ & $75 \%$ & & Median & $25 \%$ & $75 \%$ \\
\hline Control $(n=104)$ & 0.32 & 0.12 & 0.63 & & 0.88 & 0.43 & 1.46 \\
Gabazine $(n=80)$ & 0.50 & 0.25 & 1.05 & & 1.13 & 0.63 & 1.61 \\
GABA $(n=84)$ & 0.08 & 0.03 & 0.16 & & 0.40 & 0.18 & 0.69 \\
Recovery $(n=88)$ & 0.28 & 0.10 & 0.57 & & 0.80 & 0.37 & 1.17 \\
\hline
\end{tabular}

significantly $(P<0.001)$ between the groups. Dunn's method was used to compare all the conditions relative to the control group and indicated differences in the control versus gabazine conditions and control versus GABA conditions $(P<0.05 ; Q=5.863$ and $Q=4.665$, respectively), but not in the control versus recovery conditions $(P>0.05 ; Q=0.784)$. The same differences were observed in median values for the deviant stimuli (Friedman's test, $P<0.001$ ) and confirmed with Dunn's method for control versus gabazine and control versus GABA comparisons $(P<0.05 ; Q=4.087$ and $Q=5.367$, respectively), but not for the control versus recovery comparison $(P>0.05 ; Q=0.284)$. The Wilcoxon signed rank test was used to compare the median firing rate for the standard and deviant conditions. In all conditions (control, gabazine, GABA and recovery) the firing rate for the deviant stimulus was significantly larger than the firing rate for the standard stimulus $(P<0.001$ in all cases; $Z=8.122, Z=7.311, Z=7.355$ and $Z=7.637$, respectively).

The CSI value was calculated for each neuron in the control $(n=52)$ and recovery $(n=44)$ conditions, and for at least one of the two experimental conditions: (i) after gabazine application $(n=40)$, and/or (ii) after GABA application $(n=42)$. As all neurons were affected by the drug application, data were pooled regardless of CSI values. At a population level, Friedman's repeated-measures ANOVA on ranks showed significant differences between the control and both drug groups (Fig. 4A) (CSI median, control: 0.431; gabazine: 0.345; GABA: 0.613 ; recovery: $0.457 ; P<0.001$ ), which were confirmed using Dunn's method in the control versus gabazine and control versus GABA comparisons $(P<0.05$; $Q=2.41$ and $Q=2.52$, respectively). Dunn's method did not show differences between the control and recovery conditions (Fig. $4 B)(P>0.05, Q=0.11)$. To determine whether these significant differences between CSI values correlated with individual changes in the presence of the drugs, we used the bootstrap method over 1000 randomizations to estimate the $95 \% \mathrm{CI}$ of the CSI in the control condition for each neuron [Fig. $4 B$ (black whiskers indicate the $\mathrm{CI}$ )]. The analysis demonstrated that, regardless of the level of SSA, 79\% of neurons analysed $(n=41 / 52)$ showed significant changes in SSA sensitivity (i.e. gabazine generally decreased and GABA increased the degree of SSA). The application of gabazine decreased CSI levels (Fig. 4B, green triangles) in 18 of 40 neurons $(45 \%)$, increased CSI levels in two cases $(5 \%)$, and did not change them in 20 of 40 neurons $(50 \%)$. Similarly, the application of GABA increased the levels of CSI in 24 of 42 neurons (57\%) (Fig. $4 B$, red circles), produced a significant decrease in five cases $(12 \%)$, and caused no change in 13 cases $(31 \%)$. There is a direct correlation between the change in CSI and firing rate such that the larger the change in firing rate, the larger the change in CSI, independently of the drug used (Pearson's correlation, $Q=-0.548, P<0.05)$. All but 11 neurons completely recovered to control values (Fig. $4 B$, cyan triangles). Across the population, GABA effects were more noticeable when the neuron initially showed low CSI levels, whereas gabazine decreased CSI mainly for neurons with larger CSI values.

\section{Comparison of GABA and gaboxadol effects on SSA}

Seven of the 52 neurons were evaluated using GABA and gaboxadol applications, making it possible to compare the effects of each (GABA activates both $\mathrm{GABA}_{\mathrm{A}}$ and $\mathrm{GABA}_{\mathrm{B}}$ receptors, whereas gaboxadol is a $\mathrm{GABA}_{\mathrm{A}}$ selective superagonist). Repeated-measures ANOVA indicated that mean values for both the standard and deviant stimuli differed significantly $(P<0.001)$ between the groups (data not shown). The Holm-Sidak method was used to make comparisons among all the conditions and established differences in the control versus GABA and control versus gaboxadol conditions for both the standard $(P<0.05 ; t=8.105$ and $t=6.576$, respectively $)$ and deviant $(P<0.05 ; t=5.086$ and $t=4.222$, respectively $)$ stimuli, but not in the GABA versus gaboxadol conditions $(P>0.05$ for both standard and deviant stimuli). Repeated-measures ANOVA showed differences in CSI between the control group and both drug groups (mean \pm S.D. CSI, control: $0.41 \pm 0.15$; GABA: $0.59 \pm 0.21$; gaboxadol: $0.57 \pm 0.08$; recovery: $0.37 \pm 0.14$; $P<0.001)$. Holm-Sidak post hoc analysis confirmed these differences but did not show any differences between the control and recovery conditions $(P>0.05)$ or the GABA and gaboxadol conditions $(P>0.05)$. The lack of differential gaboxadol action suggests that GABA's mechanism for enhancing SSA may not be mediated only by specific extrasynaptic $\mathrm{GABA}_{\mathrm{A}}$ or $\mathrm{GABA}_{\mathrm{B}}$ receptors.

\section{Effects of gabazine and GABA on the time course of adaptation}

The effects of applications of gabazine and GABA on the temporal dynamics of adaptation during the oddball 
sequence were evaluated before, during and after drug application (Fig. 5). The time course of adaptation in all four conditions (control, gabazine, GABA and recovery) was fitted by a double exponential function defined as $f(t)=A_{\text {stst }}+A_{r} \cdot e^{-t / \tau(r)}+A_{s} \cdot e^{-t / \tau(s)}\left(r^{2}=0.89-0.95\right)$. This function contains a rapid $(r)$ and a slow $(s)$ component, after which the response reaches a steady state $\left(A_{\text {stst }}\right)$. Because of its subtle adaptation, deviant tones do not fit this function (Antunes et al. 2010; Antunes \& Malmierca, 2011). We analysed only the responses of neurons that showed significant SSA (CSI $>0.18)$ [control $(n=42)$, gabazine $(n=32), \mathrm{GABA}(n=32)$ and recovery $(n=35)]$ because the use of all the neurons in the population would have diluted these dynamics.

The rapid component did not show differences in the speed/time course of decay $[\tau(r)]$ in the control versus gabazine conditions, but did reveal significant differences between the control and GABA conditions (Table 2). Moreover, there were significant differences in the magnitude of the decay of the rapid component $\left(A_{r}\right)$ between the control and GABA conditions (Table 2, second column). For the slow component, the speed of decay $[\tau(s)]$ in the control condition differed significantly with that in the GABA condition, but not with that in the gabazine condition (Table 2, third column). Additionally, the magnitude of decay of the slow component $\left(A_{s}\right)$ was significantly lower in both the
GABA and gabazine conditions (Table 2, fourth column). Interestingly, in the GABA condition, $A_{s}$ was more pronounced (but the decrease smaller) than in the control condition. This correlated with the larger decrease in the response observed within the rapid component. Finally, the magnitude of the steady-state component $\left(A_{\text {stst }}\right)$ confirmed that the response was greatly reduced in the

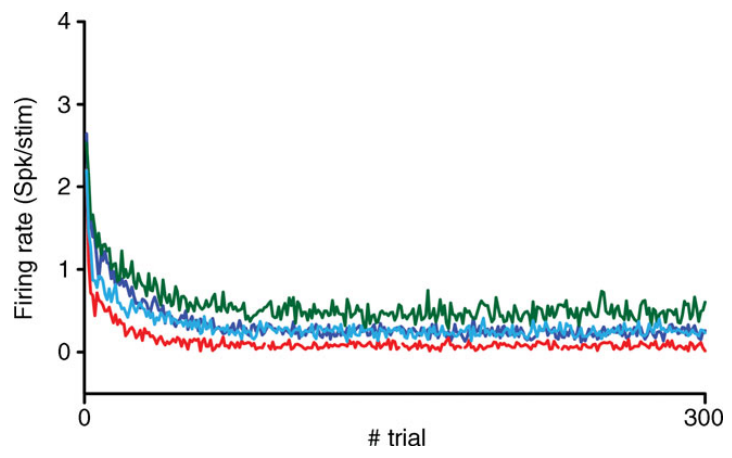

Figure 5. Time course of adaptation in medial geniculate body (MGB) neurons before, during and after the application of gabazine and GABA

Averaged population firing rate responses (spikes/stimulus) to the standard stimulus of neurons with adaptation [common specific index $(\mathrm{CSI})>0.18]$ in the control (dark blue), gabazine (green), GABA (red) and recovery (light blue) conditions.
$A$

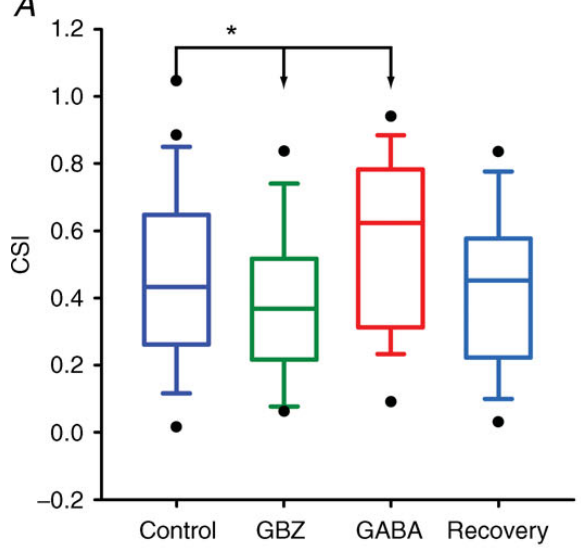

$B$

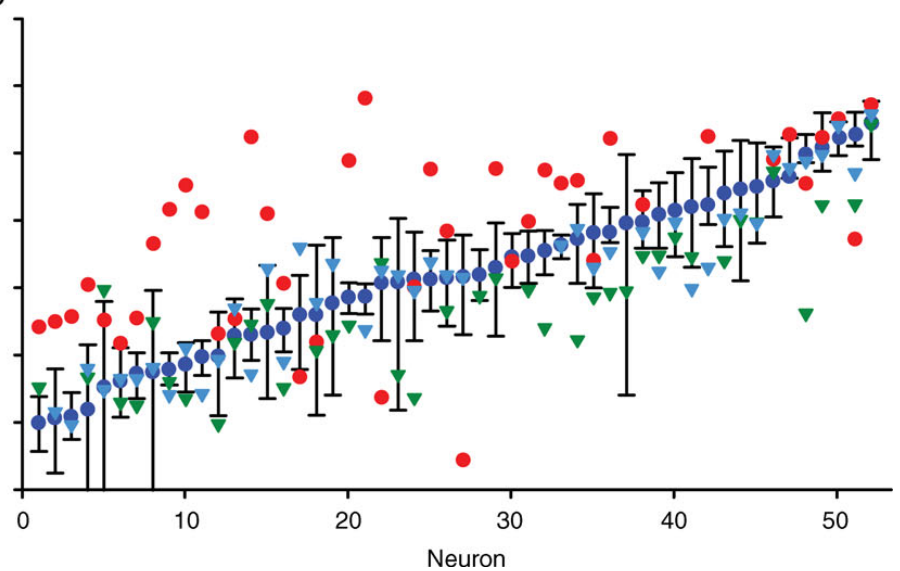

Figure 4. Stimulus-specific adaptation (SSA) quantification in medial geniculate body (MGB) neurons before, during and after the application of gabazine and GABA

$A$, distribution of common stimulus-specific adaptation index (CSI) values in the control (dark blue), gabazine (GBZ; green), GABA (red) and recovery (light blue) conditions for the population of neurons that responded properly considering our criteria (control: $n=52$; gabazine: $n=40$; GABA: $n=42$; recovery: $n=44$ ). Continuous lines across the plots represent median values. *, significant differences (between drug conditions: Friedman's test, $P<0.01$; between standard and deviant stimuli: Wilcoxon's test, $P<0.01) . B, C S I$ values for individual neurons in the control (dark blue circles), gabazine (green triangles), GABA (red circles) and recovery (light blue triangles) conditions. Error bars indicate the $95 \%$ confidence intervals of the control responses of each neuron calculated using bootstrapping. All dots outside error bars represent CSI values that are statistically different from those of the control condition. 
Table 2. Double exponential coefficients in different conditions. Superimposition with the $95 \%$ confidence interval in the control condition indicates there are no significant differences between the groups

\begin{tabular}{|c|c|c|c|c|c|}
\hline \multirow[b]{2}{*}{ Condition $\left(r^{2}\right)$} & \multicolumn{2}{|c|}{ Fast component } & \multicolumn{2}{|c|}{ Slow component } & \multirow[b]{2}{*}{ Steady-state value $\left(A_{\text {stst }}\right)$} \\
\hline & Speed: $\tau(r)(m s)$ & Decay: $A_{r}$ & Speed: $\tau(\mathrm{s})(\mathrm{ms})$ & Decay: $A_{s}$ & \\
\hline Control (0.95) & $0.96(0.67-1.25)$ & $3.02(2.00-4.04)$ & $22.38(20.79-23.96)$ & $1.37(1.29-1.44)$ & $0.28(0.27-0.29)$ \\
\hline Gabazine (0.89) & $1.13(0.71-1.55)$ & $2.85(1.80-3.89)$ & $23.83(20.98-26.69)$ & $1.16^{*}(1.05-1.27)$ & $0.48^{*}(0.47-0.49)$ \\
\hline GABA (0.95) & $0.54^{*}(0.42-0.66)$ & $8.14^{*}(4.89-11.40)$ & $15.69 *(14.42-16.96)$ & $0.83^{*}(0.78-0.88)$ & $0.06^{*}(0.06-0.07)$ \\
\hline
\end{tabular}

Values are means (95\% confidence intervals). *, statistical differences.

GABA condition in comparison with the control condition (in some cases GABA application completely suppressed responses to the standard tone) (Table 2, last column).

\section{Effects of gabazine and GABA on the latency of MGB neurons}

The impacts of gabazine and GABA application on first spike latency (FSL) in response to the standard and deviant stimuli were evaluated (control: 104; gabazine: 84; GABA: 80; recovery: 88 ). Both gabazine and GABA had a greater effect on the temporal response to deviant stimuli relative to the standard (Fig. 6; Table 3). However, no significant differences emerged between the control and drug conditions for either the standard or deviant stimuli (Friedman's test, $P=0.169$ and $P=0.083$, respectively). Generally, gabazine resulted in a small non-significant reduction in FSL in response to deviant stimuli (Fig. 6A, red dots; Table 3, fourth column), whereas GABA application increased FSL (Fig. 6B, red dots; Table 3, fourth column). Both gabazine and GABA minimally affected responses to the standard stimuli. Latency to deviant stimuli was significantly shorter than to standard stimuli in all but the GABA condition, in which response latencies to both stimuli were equalized (Wilcoxon signed rank test: control: $Z=-5.197, P<0.001$; gabazine: $Z=-6.058, P<0.001$; GABA: $Z=-1.936, P=0.053$; recovery: $Z=-3.72, P<0.001)$.

\section{Discussion}

The present study finds that activation of $\mathrm{GABA}_{\mathrm{A}}$ receptors with GABA or the selective agonist gaboxadol results in a significant increase in the level of SSA by differentially decreasing the response rate to common stimuli and having a lesser effect on the response rate to novel stimuli. Conversely, gabazine application results in a significant decrease in SSA but increases the firing rate. In general, the increase in firing rate was larger for the standard than for the deviant stimulus. The similar effects of GABA and the $\mathrm{GABA}_{\mathrm{A}} \mathrm{R}$ superagonist gaboxadol support the suggestion that GABA inhibition enhances SSA by acting preferentially at synaptic and to a lesser extent at extrasynaptic $\mathrm{GABA}_{\mathrm{A}}$ and $\mathrm{GABA}_{\mathrm{B}}$ receptors.

Previously, SSA has been studied using the oddball paradigm and the CSI in the MGB (Yu et al. 2009; Antunes et al. 2010; Antunes \& Malmierca, 2011). For consistency, the present stimulus set was chosen to facilitate comparisons with the findings of previous studies. The present work selected pairs of frequencies close to threshold and near the high-frequency edge in order to evoke the highest possible degree of SSA (Duque et al. 2012). The levels of CSI found for the neurons in our dataset $(n=52$, mean \pm S.E.M. CSI: $0.443 \pm 0.035$; median CSI: 0.431) were similar to those in other recent MGB studies using comparable conditions (frequency contrast: $\Delta \mathrm{f} \sim 0.1$; repetition rate: $4 \mathrm{~Hz}$; deviant appearance: $10 \%)$. Yu et al. (2009) demonstrated weaker SSA in MGB $(n=41$, mean \pm S.E.M. CSI: $0.154 \pm 0.020$ ), but used a slower repetition rate $(1 \mathrm{~Hz})$ and higher intensity $(70 \mathrm{~dB} \mathrm{SPL})$, which generally evokes lower SSA levels (Duque et al. 2012). Antunes and colleagues (2010), using conditions identical to those in the present study, found degrees of SSA that were larger in the non-lemniscal regions of the MGB (median CSI, MGV: 0.08; MGD: 0.37; MGM: 0.76).

In the present study, the increase in SSA level produced by the activation of $\mathrm{GABA}_{\mathrm{A}}$ Rs reflected a larger impact than that of gabazine $\mathrm{GABA}_{\mathrm{A}} \mathrm{R}$ blockade. Even considering that these larger changes of the CSI in response to GABA, in comparison with gabazine, may be an outcome of the equation for CSI (as the spike rates to the standards approach 0 in the presence of GABA, CSI will approach 1 ), the observed differences are large enough to indicate that additional factors are necessary to explain this finding. These may include: (i) the presence of both synaptic and extrasynaptic $\mathrm{GABA}_{\mathrm{A}} \mathrm{R}$ constructs; (ii) a near-threshold basal $\mathrm{GABA}_{\mathrm{A}} \mathrm{R}$ activation state, and/or (iii) an additional effect of $\mathrm{GABA}_{\mathrm{B}}$ receptors (Luo et al. 2011). Findings from the present and previous studies suggest that GABA's ability to modulate SSA is likely to be mediated by $G_{A B A}$ rather than $G_{A B A}$ receptors. Gaboxadol effects no action and does not bind to $\mathrm{GABA}_{B}$ receptors (Bowery et al. 1983). As the present results show similar effects in SSA gain after gaboxadol and GABA application, it is unlikely that these actions are 
A

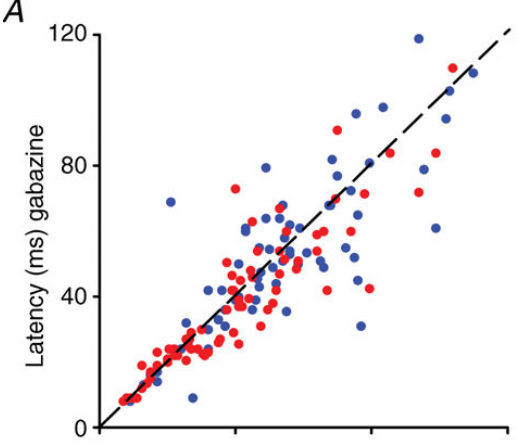

B

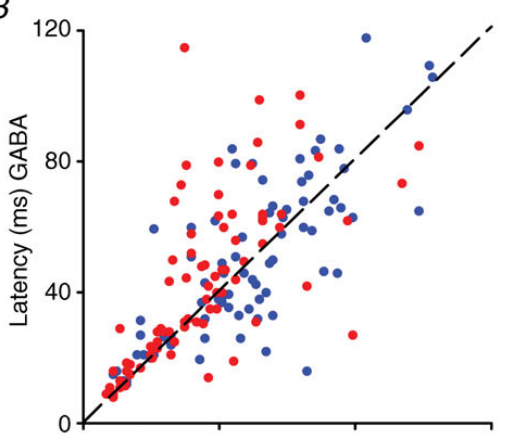

C

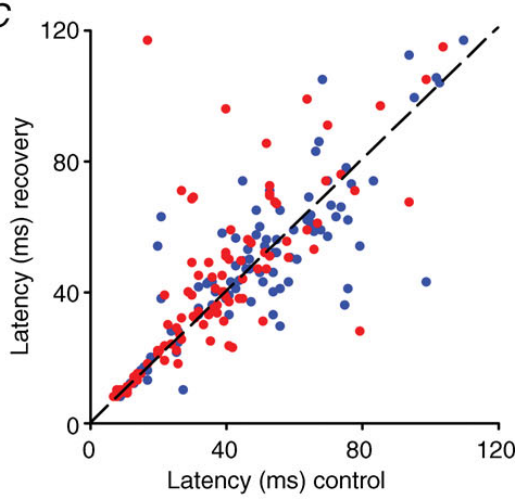

$D$

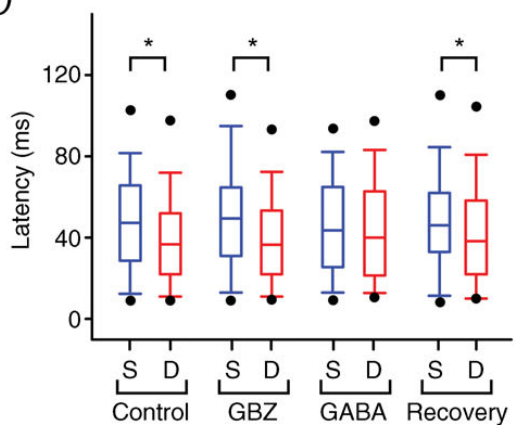

Table 3. Latencies of standard and deviant stimuli at different conditions (first spike latency)

\begin{tabular}{lccccccc}
\hline & \multicolumn{3}{c}{ Standard stimuli } & & \multicolumn{3}{c}{ Deviant stimuli } \\
\cline { 2 - 3 } \cline { 6 - 8 } Condition (drug) & Median & $25 \%$ & $75 \%$ & & Median & $25 \%$ & $75 \%$ \\
\hline Control $(n=104)$ & 47.3 & 26.0 & 65.8 & & 35.8 & 20.9 & 51.9 \\
Gabazine $(n=80)$ & 49.5 & 29.0 & 65.8 & & 29.5 & 21.0 & 51.1 \\
GABA $(n=84)$ & 43.5 & 25.6 & 65.0 & & 40.0 & 21.5 & 62.8 \\
Recovery $(n=88)$ & 44.8 & 28.0 & 62.8 & & 38.3 & 19.5 & 59.0 \\
\hline
\end{tabular}

mediated through $\mathrm{GABA}_{B}$ Rs. In addition, the fact that gabazine does not block $\mathrm{GABA}_{\mathrm{B}}$ receptors, and exerts a qualitatively smaller reduction in SSA than does GABA, reinforces the suggestion that $G_{A B A}$ receptors are not prominently involved in SSA coding. A previous study demonstrated that $\mathrm{GABA}_{\mathrm{B}}$ receptor blockade significantly reduced response habituation in the superior colliculus (Binns \& Salt, 1997). However, at least in rat, highest CSI values were found in MGM (Antunes et al. 2010), an area thought to lack $G_{A B A}$ receptors (Smith et al. 2007). With reference to (i) and (ii) above, when would an agonist be more effective than an antagonist? Relatively low endogenous levels of GABA will only activate extrasynaptic $\mathrm{GABA}_{\mathrm{A}} \mathrm{Rs}$ and, although not fully established, gabazine may require higher iontophoretic concentrations to block extrasynaptic $\mathrm{GABA}_{\mathrm{A}} \mathrm{Rs}$ relative to synaptic $\mathrm{GABA}_{\mathrm{A}} \mathrm{Rs}$ (Farrant \& Nusser, 2005; Glykys \& Mody, 2007). Thus, an agonist such as GABA or the selective agonist gaboxadol, acting at both synaptic and extrasynaptic $\mathrm{GABA}_{\mathrm{A}} \mathrm{Rs}$, may be more efficacious than the less selective antagonist gabazine.

The GABAergic projection of the TRN has been proposed as important in shaping novelty detection and/or controlling gain in MGB (Yu et al. 2009). Cortical deactivation did not significantly impact SSA in MGB (Antunes \& Malmierca, 2011), and although this manipulation does not alter the MGB-TRN-MGB inhibitory loop, it may presumably reduce the impact of the AC-TRN GABAergic input to the MGB pathway.

Figure 6. Effects of gabazine and GABA on latency responses in medial geniculate body (MGB) neurons

$A-C$, scatterplots showing the mean first spike latencies (latency, in milliseconds) of neurons to deviant (red dots) and standard (blue dots) stimuli in the control vs. gabazine $(A), G A B A(B)$ and recovery (C) conditions. Dots represent one of each frequency analysed separately in each pair of stimuli recorded with the oddball paradigm (52 neurons; 104 frequencies). D, distribution of latency values in response to standard (blue plots) and deviant (red plots) stimuli in the control, gabazine (GBZ), GABA and recovery conditions.

*, significant differences (Wilcoxon's test, $P<0.01$ ) for all groups except GABA, in which latency responses to both standard and deviant stimuli were equalized. 
As only $1 \%$ of neurons within MGB are thought to be GABAergic (Winer \& Larue, 1996), it is likely that the primary inhibitory projection impacting SSA derives from the ascending GABAergic projections from the IC (Winer et al. 1996) and the MGB-TRN-MGB circuits (Rouiller et al. 1985; Cotillon-Williams et al. 2008; Yu et al. 2009). The present data, in conjunction with those from the cortical deactivation study cited earlier (Antunes \& Malmierca, 2011), support the contention that $\mathrm{GABA}_{\mathrm{A}} \mathrm{R}$ manipulation using GABA- or gaboxadol-activated $\mathrm{GABA}_{\mathrm{A}}$ Rs may mimic ascending inhibition from IC and/or the MGB-TRN-MGB inhibitory loop.

$\mathrm{GABA}_{\mathrm{A}} \mathrm{R}$ blockade increased MGB neurons' firing rate under all conditions while decreasing CSI levels, but the temporal dynamics of adaptation were minimally affected; i.e. the adaptation function (Fig. 5) was shifted because of a general increase in firing rate. With respect to the relative impact of synaptic versus extrasynaptic $\mathrm{GABA}_{\mathrm{A}}$ Rs on SSA, exogenous application of GABA or gaboxadol similarly decreased the firing rate under all conditions, increasing the CSI levels of the MGB. If the extrasynaptic selective $\mathrm{GABA}_{\mathrm{A}} \mathrm{R}$ superagonist gaboxadol had shown a greater effect than GABA, this might have been speculated to be a primarily extrasynaptic $\mathrm{GABA}_{\mathrm{A}} \mathrm{R}$ effect (Richardson et al. $2013 b)$.

Alteration of the SSA response by $\mathrm{GABA}_{\mathrm{A}} \mathrm{R}$ manipulation was not frequency-specific: similar effects on the SSA response were apparent over a range of frequencies. Previous studies have found that GABAergic inputs impact frequency tuning through $\mathrm{GABA}_{\mathrm{A}}$ receptors (Suga et al. 1997) and alter neuronal thresholds (Cotillon-Williams et al. 2008) of MGB neurons. In the IC, Pérez-González et al. (2012) observed that $\mathrm{GABA}_{\mathrm{A}} \mathrm{R}$ manipulation altered CSI levels in a manner similar to the present MGB findings. In the present study, the GABAergic system did not generate or create SSA de novo in the MGB, but analogously to the effects observed in the IC (Ingham \& McAlpine, 2005; Pérez-González et al. 2012; Pérez-González \& Malmierca, 2012) and the visual cortex (Katzner et al. 2011), GABAergic inputs serve a significant gain control function (Robinson \& McAlpine, 2009; Isaacson \& Scanziani, 2011).

We found no differences in response latencies between the control condition and the gabazine and GABA conditions. Although somewhat unexpected, these results are in agreement with those of previous studies demonstrating that GABA inhibition has little effect on response latencies in the IC (LeBeau et al. 1996; Fuzessery et al. 2003; Sivaramakrishnan et al. 2004) and AC (Kaur et al. 2004). To the best of our knowledge, no latency analysis in the MGB in the presence of GABA receptor agonists or blockers has been performed previously. Interestingly, the changes in latency in those responses with control latencies lower than $30 \mathrm{~ms}$ appear to be much smaller than those at longer latencies. Although a detailed latency analysis of the $>30 \mathrm{~ms}$ group does not show differences between the control and the drug groups (Friedman's test, standard stimulus: $P=0.264$; deviant stimulus: $P=0.035$ ), it is tempting to speculate about a differential GABAergic effect concerning the region of the MGB: whereas MGV has short latencies, those of the MGD and MGM are longer (Calford 1983; Calford \& Aitkin 1983; Anderson et al. 2006; Anderson \& Linden, 2011). As MGB neurons maintained a shorter latency to the deviant than to the standard stimulus (Antunes et al. 2010), even during cortical deactivation (Antunes \& Malmierca, 2011), it is safe to conclude that the latency phenomenon is not of direct cortical origin nor directly related to GABA circuits. Moreover, as glycine is absent from the MGB (Aoki et al. 1988; Friauf et al. 1997), differential excitatory neuronal integration is the more likely candidate for regulating the latency of the response.

Taken together, these results suggest that in the MGB, $\mathrm{GABA}_{\mathrm{A}}$-mediated inhibition regulates SSA sensitivity in a gain control manner (i.e. by decreasing excitation to common stimuli while having a smaller effect on the response rate to novel stimuli and thus sharpening the contrast between them), thus demonstrating an 'iceberg effect' (Rose \& Blakemore, 1974; Isaacson \& Scanziani, 2011). This inhibitory effect tends to maximize the deviant to standard ratio. Assuming that excitatory inputs remain constant under the drug injections, these results may be explained by small changes that either hyperpolarize the membrane potential or increase the membrane conductance, both of which are enabled by the activation of $\mathrm{GABA}_{\mathrm{A}}$ Rs. As tonic hyperpolarization of the resting membrane potential is mediated through extrasynaptic $\mathrm{GABA}_{\mathrm{A}}$ receptors (Richardson et al. 2011, 2013b) and we have shown that gaboxadol (the extrasynaptic selective $\mathrm{GABA}_{\mathrm{A}} \mathrm{R}$ superagonist) does not exert a larger effect than GABA, tonic hyperpolarization alone is not likely to explain the iceberg effect. By contrast, as GABA may also produce a non-linear effect through shunting inhibition (i.e. by altering membrane conductance) (Borg-Graham et al. 1998; Vida et al. 2006; Mann \& Paulsen, 2007), this shunting inhibition may account for the iceberg effect. Similar results have been shown in the IC (Pérez-González et al. 2012; Pérez-González \& Malmierca, 2012). The existence of consecutive gain controls over SSA in diverse auditory nuclei (IC and MGB, so far) suggests the existence of successive hierarchical levels of processing through the auditory system that would allow the reduction of redundant information. If SSA is generated in the IC (Malmierca et al. 2009; Ayala \& Malmierca, 2013; Ayala et al. 2013), the first important GABAergic modulation will occur at this lowest level and will require additional adjustments as SSA is propagated up the auditory pathway through the MGB. The rat MGB contains one-fifth of the number of neurons present in the IC (Kulesza et al. 2002). Therefore, the role of MGB neurons may be to combine 
and integrate the adaptive properties over more inputs received than do individual IC neurons. Similar ideas have been proposed (Anderson \& Malmierca, 2013) for the role of the corticofugal modulation of SSA.

\section{References}

Anderson LA, Christianson GB \& Linden JF (2009). Stimulus-specific adaptation occurs in the auditory thalamus. J Neurosci 29, 7359-7363.

Anderson LA \& Linden JF (2011). Physiological differences between histologically defined subdivisions in the mouse auditory thalamus. Hear Res 274, 48-60.

Anderson LA \& Malmierca MS (2013). The effect of auditory cortex deactivation on stimulus-specific adaptation in the inferior colliculus of the rat. Eur J Neurosci 37, 52-62.

Anderson LA, Malmierca MS, Wallace MN \& Palmer AR (2006). Evidence for a direct, short latency projection from the dorsal cochlear nucleus to the auditory thalamus in the guinea pig. Eur J Neurosci 24, 491-498.

Antunes FM \& Malmierca MS (2011). Effect of auditory cortex deactivation on stimulus-specific adaptation in the medial geniculate body. J Neurosci 31, 17306-17316.

Antunes FM, Nelken I, Covey E \& Malmierca MS (2010). Stimulus-specific adaptation in the auditory thalamus of the anesthetized rat. PLoS One 5, e14071.

Aoki E, Semba R, Keino H, Kato K \& Kashiwamata S (1988). Glycine-like immunoreactivity in the rat auditory pathway. Brain Res 442, 63-71.

Ayala YA \& Malmierca MS (2013). Stimulus-specific adaptation and deviance detection in the inferior colliculus. Front Neural Circuits 6, 89.

Ayala YA, Perez-Gonzalez D, Duque D, Nelken I \& Malmierca MS (2013). Frequency discrimination and stimulus deviance in the inferior colliculus and cochlear nucleus. Front Neural Circuits 6, 119.

Backoff PM, Shadduck Palombi P \& Caspary DM (1999). Gamma-aminobutyric acidergic and glycinergic inputs shape coding of amplitude modulation in the chinchilla cochlear nucleus. Hear Res 134, 77-88.

Bartlett EL \& Smith PH (1999). Anatomic, intrinsic, and synaptic properties of dorsal and ventral division neurons in rat medial geniculate body. J Neurophysiol 81, 1999-2016.

Binns KE \& Salt TE (1997). Different roles for GABA $A$ and $\mathrm{GABA}_{\mathrm{B}}$ receptors in visual processing in the rat superior colliculus. J Physiol 504, 629-639.

Borg-Graham LJ, Monier C \& Fregnac Y (1998). Visual input evokes transient and strong shunting inhibition in visual cortical neurons. Nature 393, 369-373.

Bowery NG, Hill DR \& Hudson AL (1983). Characteristics of $\mathrm{GABA}_{\mathrm{B}}$ receptor binding sites on rat whole brain synaptic membranes. Br J Pharmacol 78, 191-206.

Calford MB (1983). The parcellation of the medial geniculate body of the cat defined by the auditory response properties of single units. J Neurosci 3, 2350-2364.

Calford MB \& Aitkin LM (1983). Ascending projections to the medial geniculate body of the cat: evidence for multiple, parallel auditory pathways through thalamus. J Neurosci 3, 2365-2380.
Candy JM, Boakes RJ, Key BJ \& Worton E (1974). Correlation of the release of amines and antagonists with their effects. Neuropharmacology 13, 423-430.

Caspary DM, Palombi PS \& Hughes LF (2002). GABAergic inputs shape responses to amplitude modulated stimuli in the inferior colliculus. Hear Res 168, 163-173.

Caspary DM, Schatteman TA \& Hughes LF (2005). Age-related changes in the inhibitory response properties of dorsal cochlear nucleus output neurons: role of inhibitory inputs. $J$ Neurosci 25, 10952-10959.

Clerici WJ, McDonald AJ, Thompson R \& Coleman JR (1990). Anatomy of the rat medial geniculate body: II. Dendritic morphology. J Comp Neurol 297, 32-54.

Cotillon-Williams N, Huetz C, Hennevin E \& Edeline JM (2008). Tonotopic control of auditory thalamus frequency tuning by reticular thalamic neurons. J Neurophysiol 99, 1137-1151.

Duque D, Perez-Gonzalez D, Ayala YA, Palmer AR \& Malmierca MS (2012). Topographic distribution, frequency, and intensity dependence of stimulus-specific adaptation in the inferior colliculus of the rat. J Neurosci 32, 17762-17774.

Farrant M \& Nusser Z (2005). Variations on an inhibitory theme: phasic and tonic activation of $\mathrm{GABA}_{\mathrm{A}}$ receptors. Nat Rev Neurosci 6, 215-229.

Foeller E, Vater M \& Kossl M (2001). Laminar analysis of inhibition in the gerbil primary auditory cortex. J Assoc Res Otolaryngol 2, 279-296.

Friauf E, Hammerschmidt B \& Kirsch J (1997). Development of adult-type inhibitory glycine receptors in the central auditory system of rats. J Comp Neurol 385, 117-134.

Fuzessery ZM, Wenstrup JJ, Hall JC \& Leroy S (2003). Inhibition has little effect on response latencies in the inferior colliculus. J Assoc Res Otolaryngol 4, 60-73.

Glykys J \& Mody I (2007). Activation of $\mathrm{GABA}_{\mathrm{A}}$ receptors: views from outside the synaptic cleft. Neuron 56, 763-770.

Hara K \& Harris RA (2002). The anesthetic mechanism of urethane: the effects on neurotransmitter-gated ion channels. Anesth Analg 94, 313-318.

$\mathrm{Hu}$ B (2003). Functional organization of lemniscal and nonlemniscal auditory thalamus. Exp Brain Res 153, 543-549.

Ingham NJ \& McAlpine D (2005). GABAergic inhibition controls neural gain in inferior colliculus neurons sensitive to interaural time differences. J Neurosci 25, 6187-6198.

Isaacson JS \& Scanziani M (2011). How inhibition shapes cortical activity. Neuron 72, 231-243.

Ito T, Bishop DC \& Oliver DL (2011). Expression of glutamate and inhibitory amino acid vesicular transporters in the rodent auditory brainstem. J Comp Neurol 519, 316-340.

Katzner S, Busse L \& Carandini M (2011). GABA inhibition controls response gain in visual cortex. J Neurosci 31, 5931-5941.

Kaur S, Lazar R \& Metherate R (2004). Intracortical pathways determine breadth of subthreshold frequency receptive fields in primary auditory cortex. J Neurophysiol 91, 2551-2567.

Kulesza RJ, Viñuela A, Saldaña E \& Berrebi AS (2002). Unbiased stereological estimates of neuron number in subcortical auditory nuclei of the rat. Hear Res 168, 12-24. 
LeBeau FE, Rees A \& Malmierca MS (1996). Contribution of GABA- and glycine-mediated inhibition to the monaural temporal response properties of neurons in the inferior colliculus. J Neurophysiol 75, 902-919.

Lee CC \& Sherman SM (2011). On the classification of pathways in the auditory midbrain, thalamus, and cortex. Hear Res 276, 79-87.

Luo B, Wang HT, Su YY, Wu SH \& Chen L (2011). Activation of presynaptic $\mathrm{GABA}_{\mathrm{B}}$ receptors modulates GABAergic and glutamatergic inputs to the medial geniculate body. Hear Res 280, 157-165.

Malmierca MS, Cristaudo S, Perez-Gonzalez D \& Covey E (2009). Stimulus-specific adaptation in the inferior colliculus of the anesthetized rat. J Neurosci 29, 5483-5493.

Mann EO \& Paulsen O (2007). Role of GABAergic inhibition in hippocampal network oscillations. Trends Neurosci 30, 343-349.

Näätänen R (1992). Attention and Brain Function. Lawrence Erlbaum, Hillsdale, NJ.

Paxinos G \& Watson C (2007). The Rat Brain in Stereotaxic Coordinates, 6th edn. Elsevier, Amsterdam.

Pérez-González D, Hernández O, Covey E \& Malmierca MS (2012). $\mathrm{GABA}_{\mathrm{A}}$-mediated inhibition modulates stimulus-specific adaptation in the inferior colliculus. PLOS ONE 7, e34297.

Pérez-González D \& Malmierca MS (2012). Variability of the time course of stimulus-specific adaptation in the inferior colliculus. Front Neural Circuits 6, 107.

Peruzzi D, Bartlett E, Smith PH \& Oliver DL (1997). A monosynaptic GABAergic input from the inferior colliculus to the medial geniculate body in rat. J Neurosci 17, 3766-3777.

Richardson BD, Hancock KE \& Caspary DM (2013a). Stimulus-specific adaptation in the auditory thalamus of young and aged awake rats. J Neurophysiol 110, 1892-1902.

Richardson BD, Ling LL, Uteshev VV \& Caspary DM (2011). Extrasynaptic $\mathrm{GABA}_{\mathrm{A}}$ receptors and tonic inhibition in rat auditory thalamus. PLoS One 6, e16508.

Richardson BD, Ling LL, Uteshev VV \& Caspary DM (2013b). Reduced $\mathrm{GABA}_{\mathrm{A}}$ receptor-mediated tonic inhibition in aged rat auditory thalamus. J Neurosci 33, 1218-1227.

Robinson BL \& McAlpine D (2009). Gain control mechanisms in the auditory pathway. Curr Opin Neurobiol 19, 402-407.

Rose D \& Blakemore C (1974). Effects of bicuculline on functions of inhibition in visual cortex. Nature 249, 375-377.

Rouiller EM, Colomb E, Capt M \& De Ribaupierre F (1985). Projections of the reticular complex of the thalamus onto physiologically characterized regions of the medial geniculate body. Neurosci Lett 53, 227-232.

Sivaramakrishnan S, Sterbing-D’Angelo SJ, Filipovic B, D'Angelo WR, Oliver DL \& Kuwada S (2004). GABA synapses shape neuronal responses to sound intensity in the inferior colliculus. J Neurosci 24, 5031-5043.

Smith PH, Bartlett EL \& Kowalkowski A (2006). Unique combination of anatomy and physiology in cells of the rat paralaminar thalamic nuclei adjacent to the medial geniculate body. J Comp Neurol 496, 314-334.
Smith PH, Bartlett EL \& Kowalkowski A (2007). Cortical and collicular inputs to cells in the rat paralaminar thalamic nuclei adjacent to the medial geniculate body. J Neurophysiol 98, 681-695.

Suga N, Zhang Y \& Yan J (1997). Sharpening of frequency tuning by inhibition in the thalamic auditory nucleus of the mustached bat. J Neurophysiol 77, 2098-2114.

Taaseh N, Yaron A \& Nelken I (2011). Stimulus-specific adaptation and deviance detection in the rat auditory cortex. PLoS One 6, e23369.

Ulanovsky N, Las L, Farkas D \& Nelken I (2004). Multiple time scales of adaptation in auditory cortex neurons. J Neurosci 24, 10440-10453.

Ulanovsky N, Las L \& Nelken I (2003). Processing of low-probability sounds by cortical neurons. Nat Neurosci 6, 391-398.

Vida I, Bartos M \& Jonas P (2006). Shunting inhibition improves robustness of gamma oscillations in hippocampal interneuron networks by homogenizing firing rates. Neuron 49, 107-117.

von der Behrens W, Bauerle P, Kossl M \& Gaese BH (2009). Correlating stimulus-specific adaptation of cortical neurons and local field potentials in the awake rat. J Neurosci 29 , 13837-13849.

Wang H, Turner JG, Ling L, Parrish JL, Hughes LF \& Caspary DM (2009). Age-related changes in glycine receptor subunit composition and binding in dorsal cochlear nucleus. Neuroscience 160, 227-239.

Winer JA (1985). The medial geniculate body of the cat. $A d v$ Anat Embryol Cell Biol 86, 1-97.

Winer JA \& Larue DT (1996). Evolution of GABAergic circuitry in the mammalian medial geniculate body. Proc Natl Acad Sci U S A 93, 3083-3087.

Winer JA, Saint Marie RL, Larue DT \& Oliver DL (1996). GABAergic feedforward projections from the inferior colliculus to the medial geniculate body. Proc Natl Acad Sci U $S$ A 93, 8005-8010.

Yaron A, Hershenhoren I \& Nelken I (2012). Sensitivity to complex statistical regularities in rat auditory cortex. Neuron 76, 603-615.

Yu XJ, Xu XX, He S \& He J (2009). Change detection by thalamic reticular neurons. Nat Neurosci 12, 1165-1170.

\section{Additional information}

\section{Competing interests}

None declared.

\section{Author contributions}

The experiments were performed in the Laboratory of Auditory Neurobiology, Department of Pharmacology, SIU School of Medicine (Springfield, IL, USA). D.M.C. and M.S.M. contributed to the conception and design of the experiments. D.D. collected and analysed the data. All authors contributed to the interpretation of the data and the writing of the paper, and approved the final manuscript for submission. 


\section{Funding}

This work was supported by a National Institutes of Health grant (DC000151) to D.M.C. and the Spanish Ministry of Economy and Competitiveness (MINECO) (BFU2009-07286 and EUI2009-04083) in the frame of the ERA-NET NEURON to M.S.M. D.D. held a fellowship from the Spanish Ministry of Education and Science (Ministerio de Educación y Ciencia, MEC; BES-2010-035649).

\section{Acknowledgements}

The authors thank Dr B.D. Richardson, Dr R. Cai and L.L. Ling for their help and support. 

Study III: SSA in the awake and anesthetized IC

Duque D, Malmierca MS (2014)

Stimulus-specific adaptation in the inferior colliculus of the mouse:

anesthesia and spontaneous activity effects.

Brain Structure \& Function (Epub ahead of print).

doi: 10.1007/s00429-014-0862-1. 



\title{
Stimulus-specific adaptation in the inferior colliculus of the mouse: anesthesia and spontaneous activity effects
}

\author{
Daniel Duque $\cdot$ Manuel S. Malmierca
}

Received: 3 May 2014/ Accepted: 29 July 2014

(C) Springer-Verlag Berlin Heidelberg 2014

\begin{abstract}
Rapid behavioral responses to unexpected events in the acoustic environment are critical for survival. Stimulus-specific adaptation (SSA) is the process whereby some auditory neurons respond better to rare stimuli than to repetitive stimuli. Most experiments on SSA have been performed under anesthesia, and it is unknown if SSA sensitivity is altered by the anesthetic agent. Only a direct comparison can answer this question. Here, we recorded extracellular single units in the inferior colliculus of awake and anesthetized mice under an oddball paradigm that elicits SSA. Our results demonstrate that SSA is similar, but not identical, in the awake and anesthetized preparations. The differences are mostly due to the higher spontaneous activity observed in the awake animals, which also revealed a high incidence of inhibitory receptive fields. We conclude that SSA is not an artifact of anesthesia and that spontaneous activity modulates neuronal SSA differentially, depending on the state of arousal. Our results suggest that SSA may be especially important when nervous system activity is suppressed during sleep-like states. This may be a useful survival mechanism that allows the organism to respond to danger when sleeping.
\end{abstract}

\footnotetext{
D. Duque · M. S. Malmierca

Auditory Neurophysiology Unit, Laboratory for the

Neurobiology of Hearing, Institute of Neuroscience of Castilla Y

León, University of Salamanca, C/Pintor Fernando Gallego,

1, 37007 Salamanca, Spain

M. S. Malmierca ( $\square)$

Department of Cell Biology and Pathology, Faculty of Medicine,

University of Salamanca, Campus Miguel de Unamuno,

37007 Salamanca, Spain

e-mail: msm@usal.es
}

Keywords Auditory - SSA · Anesthesia - Awake animal · Inferior colliculus · Change detection - Spontaneous activity

\section{Introduction}

The inferior colliculus (IC) is important for integrating all information arising from the auditory brainstem (Malmierca 2003; Malmierca and Ryugo 2011), but it may also play a previously unknown role in gating thalamic activity, and hence controlling cortical activation (Winer et al. 1996; Peruzzi et al. 1997; Ito et al. 2009). In recent years, significant progress has been made in the understanding of auditory subcortical plasticity (Chandrasekaran and Kraus 2010), and evidence against a strict and simple inputoutput role for the IC is abundant (Skoe et al. 2013a, b; Skoe and Kraus 2010; Malmierca et al. 2014). Some authors even suggest that the IC is analogous to the main visual cortical region in processing complexity (King and Nelken 2009).

How anesthesia may alter plasticity in the IC is an important issue since there is little doubt that anesthesia may alter some neuronal properties. For example, degradation of temporal coding (Tollin et al. 2004; Song et al. 2011) and ITD sensitivity (Fitzpatrick et al. 1995) by anesthesia has been reported in the IC. Likewise, a much higher level of spontaneous activity has been reported in the IC of awake rabbits compared with anesthetized cats (Kuwada et al. 1989) and, recently, Chung and colleagues (2014) observed that unmasking of spontaneous activity in the awake state revealed suppressive responses to electric stimulation that were rarely observed in anesthetized animals. Moreover, a reduction in response adaptation kinetics has been shown in the IC of the awake gerbil (Ter-Mikaelian et al. 2007). 
Stimulus-specific adaptation (SSA; Ulanovsky et al. 2003), the decrease that some auditory neurons show in response to repetitive sounds while maintaining responsiveness to uncommon sounds, has been extensively studied in anesthetized preparations. While SSA was originally described in the auditory cortex (AC; Ulanovsky et al. 2003), it has been shown to also occur at the levels of the midbrain and thalamus (for a review see Ayala and Malmierca 2013; Antunes and Malmierca 2014). However, most SSA experiments have been performed under anesthesia. Interestingly, the levels of SSA reported in the few experiments on awake animals (von der Behrens et al. 2009; Fishman and Steinschneider 2012; Thomas et al. 2012; Nir et al. 2013; Richardson et al. 2013) are considerably lower than those found in anesthetized animals (Malmierca et al. 2009; Antunes et al. 2010; Antunes and Malmierca 2011; Zhao et al. 2011; Duque et al. 2012, 2014; Pérez-González et al. 2012; Ayala et al. 2013). Thus, one might argue that SSA may be profoundly affected by anesthetic state or even represent an artifact due to anesthesia (Jones et al. 2012). But studies comparing SSA in awake and anesthetized using the same preparation are missing. Here we investigate what effect, if any, urethane anesthesia has on SSA in the IC of the mouse.

In the present account, we compared single-unit IC responses in awake and anesthetized mice. Our results demonstrate that SSA in the two conditions is qualitatively similar. The only significant difference between the two preparations was a much higher rate of spontaneous activity in the awake animals. Therefore, we conclude that SSA is a genuine property of some IC neurons and not simply the result of anesthesia.

\section{Materials and methods}

\section{Animals}

Experiments were performed on 28 animals, 16 awake and 12 anesthetized young-adult, male CBA/J mice (Mus musculus) aged between 2 and 6 months. The CBA/J strain exhibits normal hearing sensitivity during the first 2 years of life (Willott et al. 1988). All experimental procedures were carried out at the University of Salamanca with the approval of, and using methods conforming to the standards of, the University of Salamanca Animal Care Committee.

\section{Surgical procedures: anesthetized preparation}

Anesthesia was induced (1.5 g/kg, i.p., $20 \%$ solution) and maintained $(0.5 \mathrm{~g} / \mathrm{kg}$, i.p. given as needed) with urethane as routinely done in our lab (e.g., Malmierca et al. 2005, 2008, 2009; Hernández et al. 2005, 2006; Pérez-
González et al. 2005, 2012; Izquierdo et al. 2008; Duque et al. 2012; Ayala et al. 2013). The animal was placed in a stereotaxic frame inside a sound-attenuated room and body temperature was maintained at $38 \pm 1{ }^{\circ} \mathrm{C}$ by a heating blanket. The head was stabilized and leveled with ear bars; care was taken not to damage the eardrums. An incision was made in the scalp along the midline, and the skin was reflected laterally. A duralumin headpost ( $1 \mathrm{~g}$ ) was glued to the skull with a one-component self-etching light-cured adhesive (G-Bond $^{\mathrm{TM}}$, GC Corporation, Tokyo, Japan) and secured using a light-curing hybrid composite (Charisma ${ }^{\circledR}$, Heraeus Kulzer, Hanau, Germany). A silver ground wire was glued onto the skull over the left cerebral cortex and a craniotomy was performed to expose the right IC. After the craniotomy, the ear bars were removed and sound was delivered to the left ear canal via polypropylene tubing.

\section{Surgical procedures: awake preparation}

Awake surgical procedures followed those of Bryant and colleagues (2009) and Portfors and collaborators (Portfors et al. 2009, 2011; Holmstrom et al. 2010; Muniak et al. 2012). Animals were anesthetized with isoflurane inhalation and placed in a stereotaxic frame. The surgical procedure was the same as described above: the head was stabilized and a headpost was cemented on the skull with the same light-cured adhesives. The analgesic buprenorphine (Buprex ${ }^{\circledR}$, RB Pharmaceuticals, Berkshire, UK) was injected ip $(0.03 \mathrm{mg} / \mathrm{kg})$ and topical triple antibiotic (Dermisone $^{\circledR}$, Novartis, Barcelona, Spain) was applied to the wound. The animal was returned to its cage to recover from surgery for 2 days prior to starting electrophysiological recordings. Before the recording sessions began, the animals were acclimated to the recording chamber and were given a food reward (sweetened full cream condensed milk diluted 50:50 with water) at the end of every habituation session. During recordings, the head was immobilized by fixing the headpost to a custom-made clamp. In order to limit body movements the body was inserted in a loose fitting plastic tube. The mild sedative acepromazine (Equipromacina, Fatro Ibérica, Barcelona, Spain) was given if the mouse struggled excessively during the recording sessions $(2 \mathrm{mg} / \mathrm{kg}$, ip). If the animal continued struggling, we terminated the daily recording session.

\section{Electrophysiological recording}

Extracellular single unit responses were recorded using a tungsten electrode (1-2 M $\Omega$, Merrill and Ainsworth 1972) lowered by means of a piezoelectric microdrive (Burleigh 6000 ULN). Acoustic stimuli were delivered through a sealed acoustic system (Malmierca et al. 2009) using two electrostatic loudspeakers (TDT-EC1: Tucker Davis 
Technologies) driven by two TDT-ED1 modules. Search stimuli were pure tones ( $75 \mathrm{~ms}$ with a $5 \mathrm{~ms}$ rise/fall time) monaurally delivered under computer control using TDT System II hardware and custom software (Faure et al. 2003; Malmierca et al. 2009). The output of the system at the left ear was calibrated in situ using a free-field $1 / 2^{\prime \prime}$ condenser microphone (model 4133, Brüel \& Kjær) and a dynamic signal analyzer (Photon+, Brüel \& Kjær). The maximum output of the TDT system was flat from 1 to $40 \mathrm{kHz}$ ( $\sim 89 \pm 4.3 \mathrm{~dB}$ SPL). The highest frequency produced by this system was limited to $40 \mathrm{kHz}$. The proportion of neurons responding to frequencies above this range is relatively low ( $\sim 15 \%$, Ehret 1979; Stiebler and Ehret 1985; cf. Figure 3B in Portfors et al. 2011). The second and third harmonic components in the signal were at least $40 \mathrm{~dB}$ lower than the level of the fundamental at the highest output level (Malmierca et al. 2009). Action potentials were recorded with a BIOAMP amplifier (TDT), the $10 \times$ output of which was further amplified and bandpass-filtered (TDT PC1; $f_{c}, 500 \mathrm{~Hz}$ and $3 \mathrm{kHz}$ ) before passing through a spike discriminator (TDT SD1). Spike times were logged with a resolution of $\approx 150 \mu$ s on a computer by feeding the output of the spike discriminator into an event timer (TDT ET1) synchronized to a timing generator (TDT TG6). Generation of acoustic stimuli was exactly as described by Malmierca et al. (2009). From each isolated neuron, we measure the frequency response area (FRA) by presenting pure tones using an automated procedure with 5 stimulus repetitions at each frequency (from 1 to $40 \mathrm{kHz}$, in 25 logarithmic steps, presented randomly) and intensity (10 dB steps, presented from lower to higher intensities). The spike counts evoked at each combination of frequency and intensity were then plotted using MATLAB ${ }^{\circledR}$. We used the FRA to calculate the minimum threshold and the best frequency of response, i.e., the sound frequency that can elicit a response at the minimum intensity.

\section{Data analysis}

For the acoustic stimuli, we chose pairs of frequencies $\left(f_{1}\right.$ and $f_{2}$ ) that were $10-20 \mathrm{~dB}$ above the best frequency threshold for evaluating SSA using an oddball paradigm (Näätänen et al. 1978; Ulanovsky et al. 2003). Briefly, a train of 400 stimuli containing both frequencies $f_{1}$ and $f_{2}$ was presented under the oddball paradigm: one frequency $\left(f_{1}\right)$ was presented as the standard (90\% occurrence) while, interspersed randomly among the standards, the deviant stimuli (10\% occurrence) were presented at the second frequency $\left(f_{2}\right)$. After obtaining one data set, the relative probabilities of the two stimuli were reversed, with $f_{2}$ as the standard and $f_{1}$ as the deviant. Dot raster plots are used to illustrate the responses obtained to the oddball paradigm, plotting individual spikes (e.g., Fig. 1; red dots indicate responses to the deviant; blue dots indicate responses to the standard). Stimulus presentations are marked along the vertical axis. The responses to the standard and deviant stimuli were also expressed as spikes per stimulus in the peri-stimulus histogram (PSTH), to account for the different number of presentations in each condition, due to the different probabilities.

The amount of SSA was quantified by two indices: (1) the frequency-specific index $\operatorname{SI}\left(f_{i}\right)$, where $i=1$ or 2 , defined for each frequency as $\operatorname{SI}\left(f_{i}\right)=\left[d\left(f_{i}\right)-s\left(f_{i}\right)\right] /\left[d\left(f_{i}\right)+s\left(f_{i}\right)\right]$ and (2) the common-SSA index (CSI) defined as $\mathrm{CSI}=\left[d\left(f_{1}\right)+d\left(f_{2}\right)-s\left(f_{1}\right)-s\left(f_{2}\right)\right] /\left[d\left(f_{1}\right)+d\left(f_{2}\right)+\right.$ $\left.s\left(f_{1}\right)+s\left(f_{2}\right)\right]$, where $d(f)$ and $s(f)$ are responses to each frequency $f_{1}$ or $f_{2}$ when they were the deviant $(d)$ or standard (s) stimulus (Ulanovsky et al. 2003; Malmierca et al. 2009). The SI reflects the extent to which the response to a given frequency as standard is suppressed respect to the same frequency as deviant, i.e. is a frequency-specific index for SSA. CSI reflects the same effect but for a pair of frequencies, demonstrating that the SSA levels observed in the SI are extensible to other frequencies of the neuron, i.e. provides an approximate neuronal level of SSA. The values of both indices range from -1 to +1 , being positive if the response to the deviant stimulus is greater. The same paradigm was repeated varying the interstimulus interval [ISI $=125 \mathrm{~ms}$ $(8 \mathrm{~Hz}), 250 \mathrm{~ms}(4 \mathrm{~Hz})$ and $1000 \mathrm{~ms}(1 \mathrm{~Hz})]$ and the frequency contrast $(\Delta f)$ between the standard and deviant stimulus $[\Delta f=0.04$ ( 0.057 octaves), 0.10 (0.141 octaves) and $0.37(0.526$ octaves $)]$; where $\Delta f=\left(f_{2}-f_{1}\right) /\left(f_{2} \times f_{1}\right)^{1 / 2}$.

Since the levels of spontaneous activity (SR) in the awake animal are notably high, we considered two different approaches for evaluating SSA. At first, we analyzed the response, adjusting the limits of the response analysis window based on the shape of the PSTH-considering only the evoked response to the sound-and trying to avoid the inclusion of the SR in the response window used for the analysis of the SSA. In the second approach, considering that the SR was constant even during the presentation of the tones, we computed the SSA indices, but directly subtracting the SR. To do so, SR was estimated with a fixed window established $50 \mathrm{~ms}$ before the presentation of the stimuli while we presented the oddball paradigm (50 $\mathrm{ms} \times 400$ trials $=20 \mathrm{~s}$ sample window $)$. Then, a driven rate was obtained by subtracting the averaged SR from the evoked firing rate in the same time windows used before to evaluate SSA (driven rate $=$ firing rate $-\mathrm{SR}$ ). The subtraction of the SR was directly done bin by bin (evoked responses in spikes/s minus spontaneous activity in spikes/s). The obtained driven rate was then used to calculate again the levels of SSA in each condition. It should be noticed that because the calculation of SR is not intended to include evoked activity (especially at repetition rates of $8 \mathrm{~Hz}$ ), cases that showed a likely rebound-off 

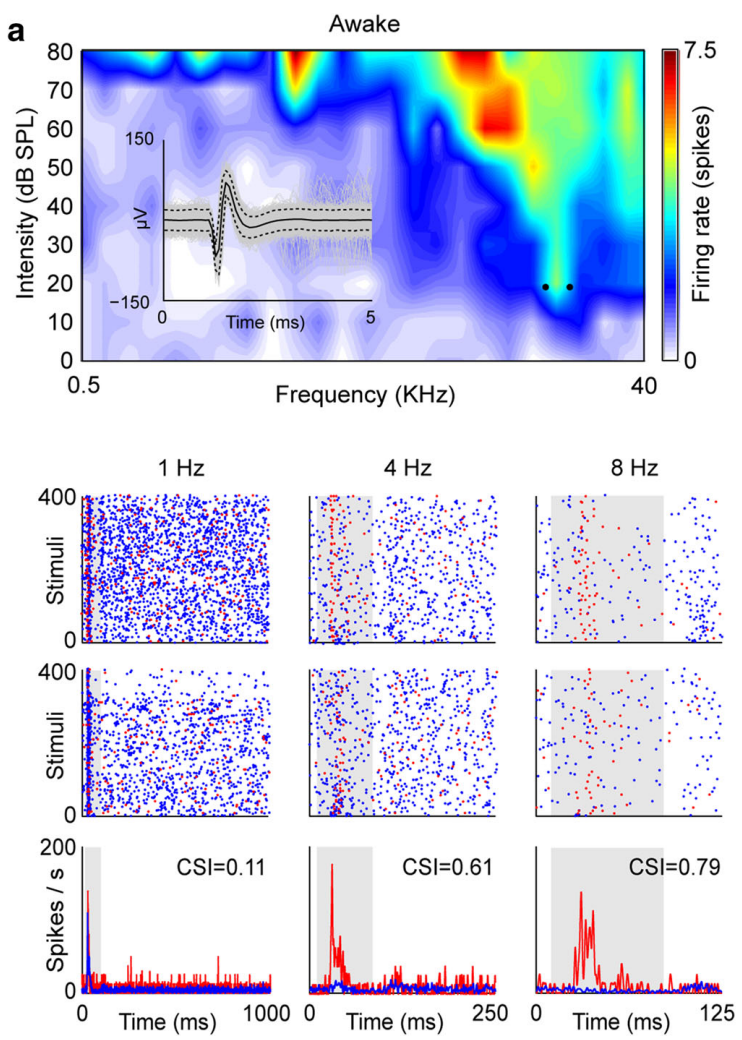

Fig. 1 Single-unit responses in the IC of the awake (a) and the anesthetized (b) mouse. Top panels show FRAs of neurons in the IC that exhibited high CSI values. Black dots over the FRAs indicate the pair of frequencies selected for analyzing SSA $(\Delta f=0.37)$. Insets in the FRAs show waveforms of the spikes recorded from these single units. Below the FRAs, dot raster plots are illustrated for the oddball paradigm under three different repetition rates: $1 \mathrm{~Hz}$ (left column), $4 \mathrm{~Hz}$ (middle column) and $8 \mathrm{~Hz}$ (right column). In the top row $f_{1}$ and

activity (i.e., post-stimulus suppression or forward masking) were not included in the analysis.

Classification of the type of response was based on previous reports (Rees et al. 1997; Duque et al. 2012): on, long-latency on, on-sustained, sustained, pauser and onoff. On responses show a robust response confined to the first $40 \mathrm{~ms}$ of the stimulus. Long-latency on responses started 50-80 ms after the beginning of the tone. On-sustained responses exhibit a clear on response followed by a sustained portion, with at least a $50 \%$ decrease of firing rate compared with the on portion. Sustained responses show a constant response that lasts $50 \mathrm{~ms}$ or more. Pauser responses show a cessation of firing rate between a clear on and a sustained portion. On-off responses had two different components, with an on and off portion (after the end of the stimulus).
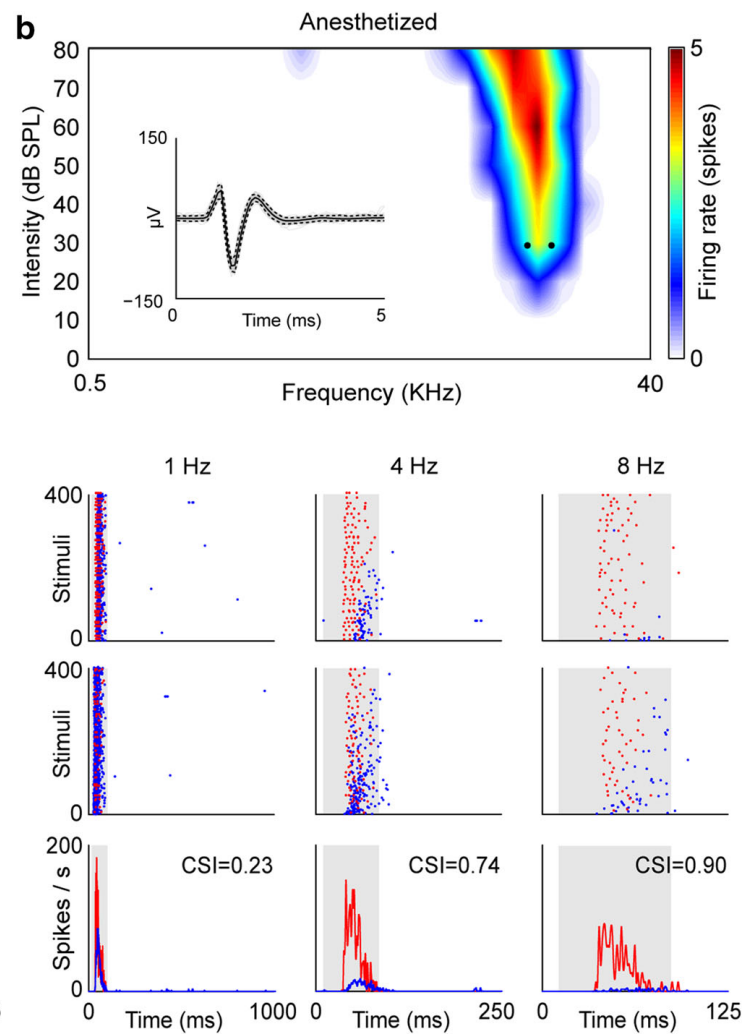

$f_{2}$ are the standard and deviant stimuli, respectively (awake: 18.86 and $27.28 \mathrm{kHz}$; anesthetized: 15.80 and $22.87 \mathrm{kHz}$ ). In the middle row, in the reverse condition, $f_{2}$ and $f_{1}$ are the standard and deviant stimuli, respectively (awake: 27.28/18.86 kHz; anesthetized: $22.87 /$ $15.80 \mathrm{kHz}$ ). In the bottom row, PSTHs show averaged responses for both conditions: deviant (red) and standard (blue). CSI values obtained in each condition are shown as insets in the PSTHs. Shaded backgrounds indicate the duration of the stimulus

\section{Histology}

Neuron location was assigned to each of the main IC subdivisions based on stereotaxic coordinates (Franklin and Paxinos 2007), response properties (Egorova et al. 2001; Portfors et al. 2011; Malmierca and Ryugo 2011) and by reconstruction of the electrode tracks from the position of electrolytic lesions (14 $\mu \mathrm{A}$ for $10 \mathrm{~s})$ in Nissl-stained brain sections. Histological procedures were as described by Duque et al. (2012).

\section{Results}

To characterize SSA in the mouse, we recorded responses from 93 well-isolated single neurons in the IC under an 
oddball paradigm. Fifty-four units were recorded from 16 awake mice and 39 units from 12 anesthetized mice. For both populations, we determined the basic temporal and spectral response properties of each neuron and chose a pair of frequencies within the FRA to evaluate SSA under different conditions (i.e., variations in frequency contrast and repetition rate). Our major goal was to study the effect of anesthesia on SSA sensitivity in the IC of the mice. In the following, we will compare the levels of SSA, the neuronal response properties and the levels of spontaneous activity of IC neurons in the awake and the urethaneanesthetized mouse.

SSA and response properties in the awake and anesthetized preparation

Since there were no available SSA data for the IC in mouse, and for purposes of comparison, we performed the same experiments and analysis in two different preparations: awake and anesthetized. Figure 1a shows a FRA of an individual neuron in the awake preparation while Fig. 1b shows a FRA of an individual neuron in the anesthetized preparation. The black dots over the FRAs indicate the frequencies and the intensity chosen for the oddball paradigm (Fig. 1a: 18.86 and $27.28 \mathrm{kHz}$ at $20 \mathrm{~dB}$ SPL; Fig. 1b: 15.80 and $22.87 \mathrm{kHz}$ at $30 \mathrm{~dB}$ SPL). The neurons illustrated exhibit significant SSA, showing a cessation of the responses to the repetitive stimuli (Fig. 1a, b: blue dots in the dot rasters, especially at 4 and $8 \mathrm{~Hz}$ ) while the responses to the deviant stimuli are maintained (Fig. 1a, b: red dots in the dot rasters). The normalized response in each condition is depicted by the corresponding PSTHs (Fig. 1a, b, bottom row); the average response to the deviant stimuli (red traces) is higher than the average response to the standard stimuli (blue traces) in all conditions. The only readily visible difference in the dot rasters (and also in the FRAs) between the awake and anesthetized preparation is the great amount of spontaneous activity (SR) observed in the awake preparation (Fig. 1a). Despite the SR, the levels of SSA depicted by the common SSA index (CSI, insets in the PSTHs) are comparable between the awake and the anesthetized preparation.

We quantified and compared the degree of neuronal adaptation in the awake and the anesthetized preparation by computing the CSI and the frequency-specific SSA index (SI, Fig. 2) under several experimental conditions. The overall degree of SSA was characterized at a standard condition with a frequency contrast $(\Delta f)$ of 0.37 and a repetition rate of $4 \mathrm{~Hz}$ (awake: $n=31$; anesthetized: $n=29$ ). Under this condition, CSI levels in the awake preparation range from -0.017 to 0.854 with an average of $0.43 \pm 0.24$ (mean $\pm \mathrm{SD}$ ). In the anesthetized preparation the levels show a comparable range from -0.043 to 0.985 with a similar average level (Table 1, mean \pm SD $0.48 \pm 0.33$ ). A CSI cut-off value of +0.18 was defined as significant SSA based on previous data (Antunes et al. 2010; Duque et al. 2012, 2014). Using this criterion, in the awake preparation 25 neurons $(81 \%)$ showed significant SSA, while the remaining $6(19 \%)$ did not. In the anesthetized preparation, 21 neurons $(73 \%)$ showed significant SSA, while the remaining $8(27 \%)$ lacked significant SSA (Table 1). Twenty-nine of the 39 neurons recorded in the anesthetized preparation were histologically localized. Anatomical analysis revealed that 6 neurons were located in the central nucleus of the IC (lemniscal), while 23 were located in the collicular cortices (non-leminscal): 4 in the dorsal, 5 in the rostral and 14 in the lateral cortex (data not shown). Neurons located in the collicular cortices showed the largest degree of SSA (mean $\mathrm{CSI}_{\text {non-lemniscal }}$ : $0.70 \pm 0.24$; mean $\mathrm{CSI}_{\text {lemniscal }}$ : $0.36 \pm 0.27$ ). Four of the seven neurons that lacked SSA were from the central nucleus, supporting previous findings that SSA is biased towards the non-lemniscal IC regions. On the other hand, recordings from awake animals limit the ability to localize individual recorded neurons. However, we recovered lesions from 7 of the 16 mice at the end of the last recording session, which allowed us to confirm that we were also biased towards the non-lemniscal regions of the IC.

To quantify the adaptation at the population level we compared the SSA indices across different conditions and between the two preparations. The SI is depicted in Fig. 2 for different conditions (awake: burgundy dots and crosses; anesthetized: green dots and crosses). These scatter plots show the individual SI values for each frequency pair and demonstrate significant SSA when the values are located in the upper right quadrant. Crosses represent the median CSI values while the mean CSI values are also indicated as insets in each condition. As expected from previous studies (Ulanovsky et al. 2003; Malmierca et al. 2009; Antunes et al. 2010), SSA values are positively correlated with the frequency contrast and the repetition rate in both preparations (two-way ANOVA; awake: $F_{\Delta f}=6.62, p=0.002$ $F_{\mathrm{Hz}}=14.85, p<0.001$; no interaction effect: $F=0.72$, $p=0.58$; post hoc Tukey test confirmed differences between $\Delta f=0.37$ and $\Delta f=0.04-0.1$ and between 1 and $4-8 \mathrm{~Hz} ; \quad p<0.05 ; \quad$ anesthetized: $\quad F_{\Delta f}=9.37$, $p<0.001 ; F_{\mathrm{Hz}}=11.32, p<0.001$; no interaction effect: $F=0.30, p=0.88$; post hoc Tukey test confirmed differences between $\Delta f=0.37$ and $\Delta f=0.04-0.1$ and between 1 and $4-8 \mathrm{~Hz} ; p<0.05)$. However, at both preparations, SSA is still present at a small frequency contrast $(\Delta f=0.04)$ and at a repetition rate as low as $1 \mathrm{~Hz}$. No differences in CSI were found at any condition between the awake and the anesthetized preparation (Mann-Whitney rank sum test, $p>0.05$ for all conditions). Despite not 
Fig. 2 Population SSA

comparison between awake and anesthetized mouse. Scatter plots of the $\operatorname{SI}\left(f_{1}\right)$ versus $\operatorname{SI}\left(f_{2}\right)$, for the different $\Delta f$ (top row: 0.04; middle row, 0.1 ; bottom row: 0.37 ) and ISIs (left column: $1 \mathrm{~Hz}$; middle column: $4 \mathrm{~Hz}$; right column: $8 \mathrm{~Hz}$ ). Pairs of frequencies obtained in the awake (burgundy dots) and anesthetized (green dots) preparations are shown. [For comparison, we also show SSA data values from previous experiments in the anesthetized rat (light violet crosses) from Malmierca et al. 2009, and Zhao et al. 2011]. The burgundy (awake) and green (anesthetized) crosses indicate the median and the 25th-75th interquartile range for each axis. As each neuron was tested using different combinations of parameters, individual neurons may be represented in more than one panel. Median CSI values for each condition are shown as insets in each scatter plot

Table 1 Neuronal responses in the awake and the anesthetized preparations

${ }^{a}$ Average firing rate response $20 \mathrm{~dB}$ above the best frequency threshold

b Data from Duque et al. (2012)

${ }^{\mathrm{c}}$ Data from Malmierca et al. (2009)

d Data from Perez-Gonzalez et al. (2012)
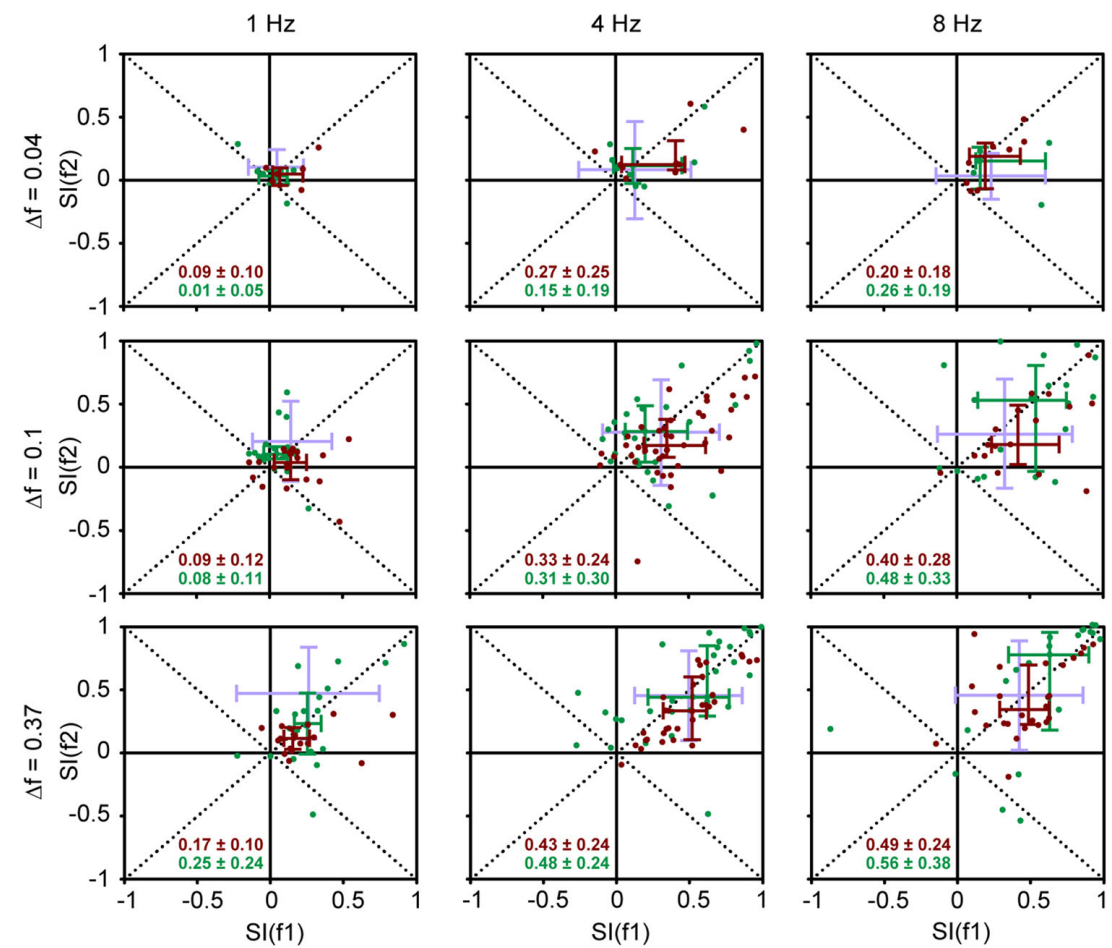

\begin{tabular}{|c|c|c|c|}
\hline \multirow[t]{2}{*}{ Preparation } & \multicolumn{2}{|l|}{ Mice } & \multirow{2}{*}{$\begin{array}{l}\text { Rat } \\
\text { Anesthetized }\end{array}$} \\
\hline & Awake & Anesthetized & \\
\hline$n$ (all neurons) & 79 & 46 & $95^{\mathrm{b}}$ \\
\hline \multicolumn{4}{|l|}{ Type of FRA } \\
\hline V-shaped (\%) & $12(15)$ & $13(28)$ & $20(21)^{\mathrm{b}}$ \\
\hline Non-V-shaped (\%) & $50(63)$ & $33(72)$ & $75(79)^{\mathrm{b}}$ \\
\hline 'Inhibitory’ (\%) & $17(22)$ & $0(0)$ & $0(0)^{\mathrm{b}}$ \\
\hline Mean firing rate $(\mathrm{spk} / \mathrm{s})^{\mathrm{a}}$ & $94.27 \pm 71.19$ & $70.12 \pm 43.6$ & $69.22 \pm 52.72^{\mathrm{b}}$ \\
\hline Mean spontaneous activity (spk/s) & $7.16 \pm 9.29$ & $0.98 \pm 1.86$ & $1.47 \pm 5.01^{\mathrm{b}}$ \\
\hline$n(\Delta f=0.37$ at $4 \mathrm{~Hz})$ & 31 & 29 & $69^{c}$ \\
\hline SSA $(\mathrm{CSI} \geq 0.18)(\%)$ & $25(81)$ & $21(73)$ & $52(75)^{\mathrm{c}}$ \\
\hline Non-SSA (CSI <0.18) (\%) & $6(19)$ & $8(27)$ & $17(25)^{\mathrm{c}}$ \\
\hline Mean CSI \pm SD & $0.43 \pm 0.24$ & $0.48 \pm 0.33$ & $0.46 \pm 0.32^{\mathrm{c}}$ \\
\hline Median CSI (25th-75th percentile) & $0.40(0.27-0.61)$ & $0.49(0.14-0.77)$ & $0.47(0.17-0.80)^{\mathrm{c}}$ \\
\hline Mean latency difference (ms) & $4.34 \pm 6.59$ & $9.04 \pm 10.34$ & $7.54 \pm 14.44^{\mathrm{c}}$ \\
\hline \multicolumn{4}{|l|}{ Adaptation values (mean $\pm 95 \%$ c.i.) } \\
\hline$\tau(r)($ trial $)$ & $0.86 \pm 0.38$ & $0.58 \pm 0.33$ & $0.92 \pm 0.13^{\mathrm{d}}$ \\
\hline$A_{r}(\mathrm{spk} /$ trial $)$ & $5.00 \pm 2.77$ & $7.68 \pm 7.47$ & $9.48 \pm 1.56^{\mathrm{d}}$ \\
\hline$\tau(\mathrm{s})$ (trial) & $34.27 \pm 8.58$ & $34.71 \pm 14.23$ & $44.54 \pm 6.26^{\mathrm{d}}$ \\
\hline$A_{s}(\mathrm{spk} /$ trial $)$ & $0.64 \pm 0.11$ & $0.34 \pm 0.10$ & $0.68 \pm 0.07^{\mathrm{d}}$ \\
\hline$A_{\text {stst }}(\mathrm{spk} /$ trial $)$ & $1.13 \pm 0.02$ & $1.04 \pm 0.02$ & $0.36 \pm 0.01^{\mathrm{d}}$ \\
\hline
\end{tabular}




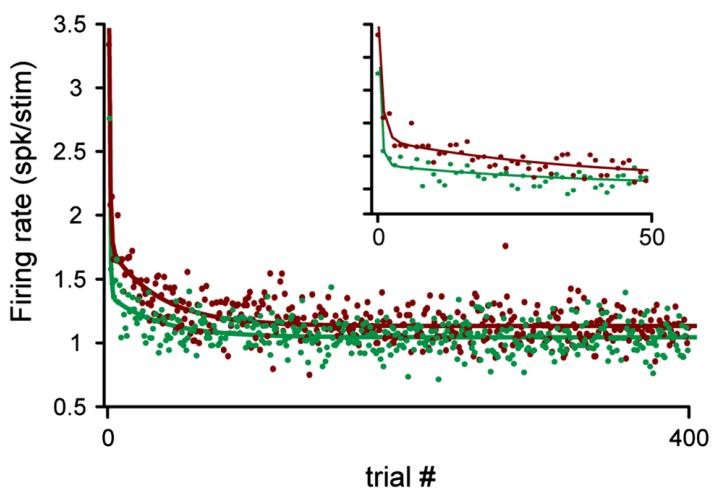

Fig. 3 Time course of adaptation in the awake and the anesthetized mouse. Averaged population firing rate responses (spikes/stimulus) to the standard stimulus at the condition with a $\Delta f=0.37$ at $4 \mathrm{~Hz}$ for the awake (burgundy, $n=62$ ) and the anesthetized (green, $n=58$ ) preparations. The upper right inset shows the first 50 trials extended

being statistically different, we found slightly different values at $\Delta f=0.1$ at $8 \mathrm{~Hz} ; \Delta f=0.37$ at $4 \mathrm{~Hz}$ and $\Delta f=0.37$ at $8 \mathrm{~Hz}$ (Fig. 2).

To better understand the dynamics of adaptation over time, we plotted the responses to the standard tone in the condition with $\Delta f=0.37$ at $4 \mathrm{~Hz}$ over the consecutive trials (Fig. 3, awake preparation in burgundy trace: $n=62$; anesthetized preparation in green trace: $n=58$ ). We fitted the responses with a double exponential function defined as $f(t)=A_{\text {stst }}+A_{r} \cdot e^{-t / \tau(r)}+A_{s} \cdot e^{-t / \tau(s)}$ (Perez-Gonzalez and Malmierca 2012; Perez-Gonzalez et al. 2012; Duque et al. 2014). The responses to the deviant tones, because of minimal adaptation, do not fit this function and for this reason they were not depicted in the figure. The response to the standard stimulus is reduced immediately after the first stimulus trials in both preparations (Fig. 3). This double exponential function contains both a rapid $(r)$ and a slow $(s)$ component, after which the response reaches a steady state $\left(A_{\text {stst }}\right)$. In terms of magnitude, the fast component, which occurs during the first trials, presents the largest degree of adaptation. The slow component defines the final steady-state response. Comparing the time course of adaptation to the standard stimuli between the awake and the anesthetized preparation, only the magnitude of the slow component $\left(A_{s}\right)$ and the asymptote of the curve $\left(A_{\text {stst }}\right)$ were statistically different (Table 1).

Since these higher time course of adaptation indices could imply that the firing rate was different in the awake and the anesthetized preparation, we compared the basic temporal and spectral responses of the recorded units in both conditions. We found that the firing rate, the PSTH response type and the response latencies were similar to those recorded in the awake preparation. No differences in firing rate at a common point in the FRA (we calculate the average firing rate response $20 \mathrm{~dB}$ above the best frequency threshold) were found between the awake and the anesthetized preparation (Table 1; Mann-Whitney rank sum test, $T=2151, p=0.13)$. Next, we characterized the basic temporal and spectral responses of the recorded units in an attempt to determine whether there is a correlation between SSA and any of these features, including PSTH response type and response latency. We classified the response types of the units according to the PSTH shape in response to the standard condition $\Delta f=0.37$ at $4 \mathrm{~Hz}$ as: on, long-latency on, on-sustained, sustained, pauser and on-off responses (see "Materials and methods"). In the awake preparation, the most common type found in our sample was the on response ( 15 units, $48 \%$ ), followed by the on-sustained ( 9 units, $29 \%$ ) and the pauser (5 units, $16 \%)$ types. One sustained unit $(3 \%)$ and one on-off response $(3 \%)$ completed our data set. The highest levels of SSA were seen in the on and on-sustained units, while pauser and sustained units showed the weakest levels of SSA (one-way ANOVA; $p=0.031$ ). In the anesthetized preparation, on responses (16 units, $55 \%$ ) were also the most common type of response, followed by the on-sustained (7 units, $24 \%$ ), sustained ( 3 units, $10 \%$ ), pauser (2 unit, $7 \%$ ), and long-latency on responses (1 units, $4 \%$ ). No on-off responses were found in our anesthetized data set. Thus, the proportion of response types do not vary between the awake and the anesthetized preparation (Chi square test for sampling distributions, $\chi^{2}=4.5$, $p=0.479$ ), although it is difficult to make definitive conclusions due to the small number of neurons in our sample. Finally, we also evaluated the effects on spike latency by computing the latency difference, defined as the difference between the median first spike latency to the standard and the deviant condition for each frequency. Across the population, a high SSA level is correlated with a large latency difference (Spearman rank order correlation coefficient: $0.36, p<0.001$ ). Interestingly, no differences in latency difference were found as a function of repetition rate or frequency contrasts in the awake preparation (twoway ANOVA; $F_{\Delta f}=0.82, \quad p=0.44 ; \quad F_{\mathrm{Hz}}=2.09$, $p=0.13$; no interaction effect: $F=1.09, p=0.36$ ) while they were evident in the anesthetized preparation (two-way ANOVA; $F_{\Delta f}=10.42, p<0.001 ; F_{\mathrm{Hz}}=3.01, p=0.05$; no interaction effect: $F=0.93, p=0.45$; Tukey test confirmed differences between $\Delta f=0.37$ and $\Delta f=0.04-0.1$ and between 1 and $8 \mathrm{~Hz} ; p<0.05)$. At some conditions, latency difference was larger in the anesthetized mice compared with the awake ones (e.g. Table 1; Mann-Whitney rank sum test for $\Delta f=0.37$ at 1 and $4 \mathrm{~Hz}, p=0.001$ ), but those differences did not generalize to all the conditions (Mann-Whitney rank sum test, 
$p>0.05)$. Such small latency difference in the awake animal is likely due to the random integration of spontaneous spikes into the analysis window.

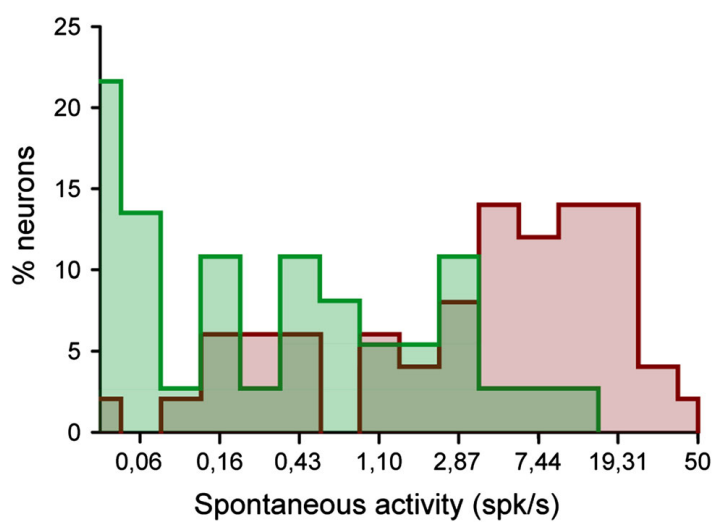

Fig. 4 Histogram of the SR in the awake and the anesthetized mouse. Note that the $x$-axis is logarithmic to account for the great difference in SR between the anesthetized (green histogram) and the awake preparation (burgundy histogram)
Urethane anesthesia decreases spontaneous activity

Even simple visual inspection suggested a high SR for the majority of units during the recording sessions in the awake mouse $(68 \%$ of the neurons recorded showed a SR larger than 2 spikes/s, Fig. 4, burgundy histogram). At a population level, the range of SR was from 0.11 to 28.31 spikes/ $\mathrm{s}$ and the mean SR was $7.16 \pm 9.29$ spikes/s (Table 1). On the other hand, the mean SR in the anesthetized preparation was very low or negligible: $0.98 \pm 1.86$ spikes/s (Fig. 4, green histogram; SR range from 0 to 2.51 spikes/s). Only $16 \%$ of the neurons recorded showed a SR larger than 2 spikes/s and $46 \%$ of the neurons showed no SR at all. Thus, median SR in the awake preparation was significantly higher than that seen in the anesthetized mouse (Anesthetized: 0.222 spikes/s; Awake: 3.8 spikes/s; MannWhitney rank sum test, $p<0.001$ ). Moreover, for some units in the awake preparation the SR was so high that no sound-evoked response could be distinguished out of the SR. In those cases, which account for almost a quarter of our sample (Table 1, 17/79; $22 \%$ ), the SR allowed us to detect suppression of responses to different combinations of frequencies and intensities, resulting in a distinct and
Fig. 5 Examples of iFRAs in the IC of the awake mouse. Three examples of inhibitory frequency response areas (iFRA) are shown in the left panel. Averaged PSTHs obtained for each neuronal example are shown in the right panel next to each iFRA to show that no excitatory response was elicited before the suppression of the SR. Dotted lines indicate the mean averaged SR for each neuronal example. Firing rate values for each iFRA shared the $y$-axis of the averaged neuronal PSTH

\section{a}
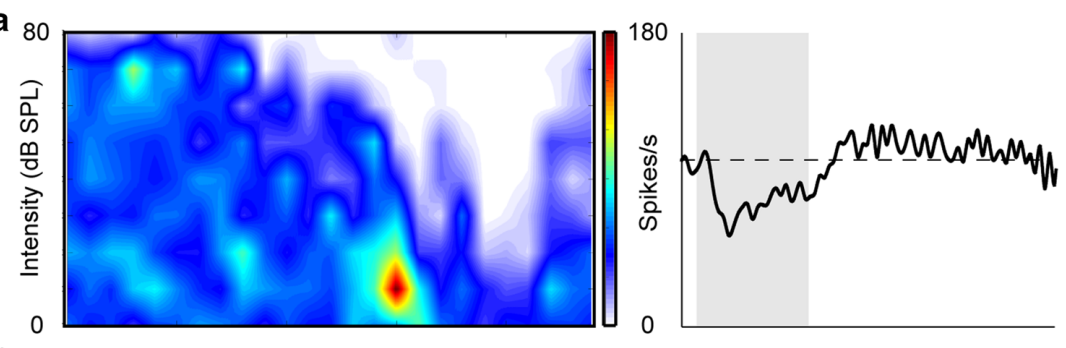

b
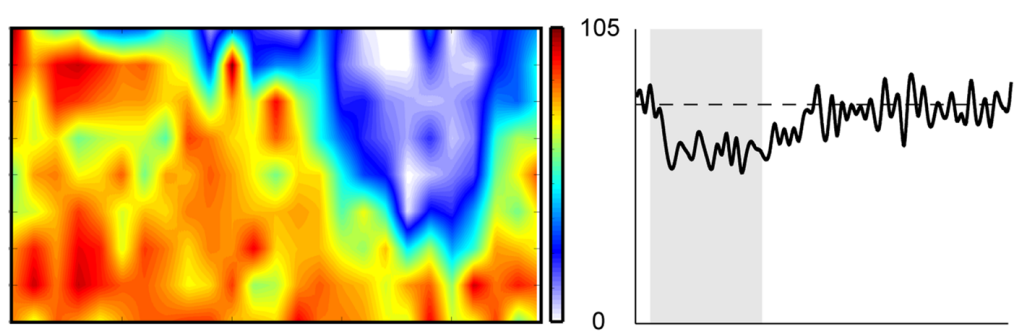

c
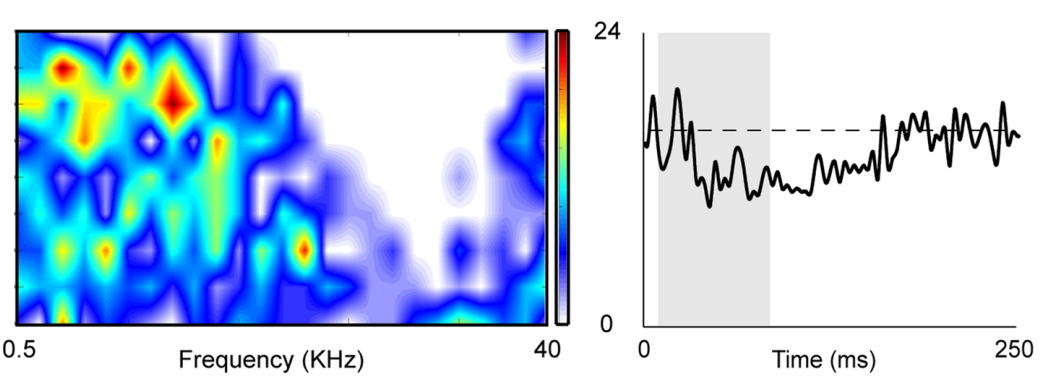
unambiguous 'inhibitory' FRA (iFRA: Fig. 5). Unfortunately, due to the lack of driven activity, we could not test those neurons showing iFRAs with the oddball paradigm, nor they did show any sign of SSA in the SR region. The significant difference in SR may explain why we did not find iFRAs in the anesthetized preparation (Table 1,0/45).

Since the high SR rates in the awake preparation might have affected the levels of SSA in some conditions (Fig. 2, e.g. $\Delta f=0.37$ at $4 \mathrm{~Hz}$ ), we next proceeded to extract the SR and reevaluate the levels of CSI of the neurons recorded for the awake preparation. To do that, we subtracted the SR (see "Materials and methods"), in an attempt to mimic the anesthetized preparation. Figure 6 a shows the same frequency pair example as in Fig. 1a, with a $\Delta f=0.37$ at $4 \mathrm{~Hz}$. The depicted PSTHs are shown in Fig. 6b, c, with and without SR, respectively. Interestingly, the subtraction of the SR demonstrates a distinct increase in the CSI level. In order to shed light on the role, if any, of the SR in the modulation of SSA, we plotted the CSI values of each a
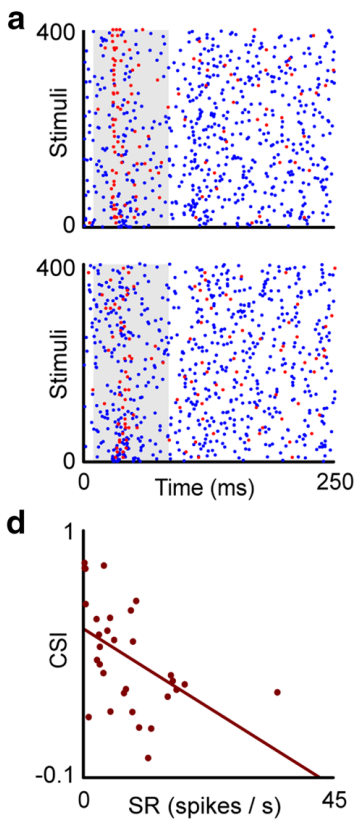
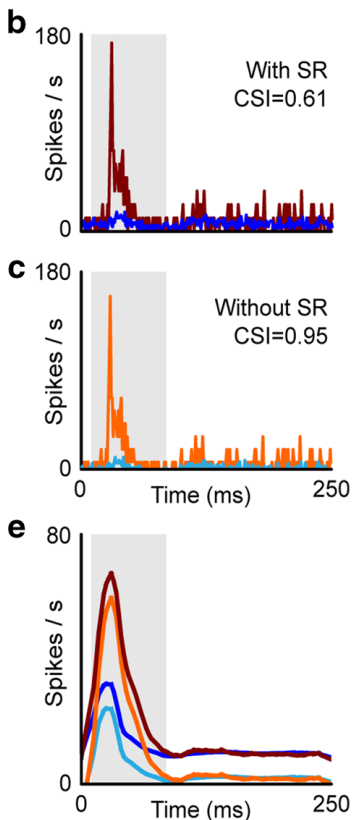

Fig. 6 Relation between spontaneous activity and SSA. a Dot raster plots for the same frequency pair illustrated in Fig. 1a, with a $\Delta f=0.37$ at $4 \mathrm{~Hz}$. b PSTHs for the averaged responses for the frequency pair before subtracting the spontaneous rate (SR). c PSTHs for the averaged responses for the frequency pair after subtracting the SR. d Correlation between the SR and the level of CSI in the standard condition, at $\Delta f=0.37$ and $4 \mathrm{~Hz}(n=31)$. e Grand average for the standard condition, with a $\Delta f=0.37$ at $4 \mathrm{~Hz}(n=31)$. Deviant and standard responses for the neurons before the subtraction of the SR are shown as burgundy and blue lines, respectively. Deviant (orange) and standard (light blue) responses after the subtraction of the SR are also shown neuron as a function of its SR and found that they were inversely correlated (Fig. 6d, $\Delta f=0.37$ at $4 \mathrm{~Hz}$, Spearman rank order correlation coefficient: $-0.58, p<0.001)$. The same analysis was performed to the neurons in the anesthetized preparation, and a similar trend occurred. However, here the correlation was not significant, most probably because many neurons in this group totally lacked of SR and the sample of neurons with SR turned very small ( $n=9$; Spearman rank order correlation coefficient: $-0.45, p=0.204$, data not shown). Moreover, despite the large variability in SR across the population, the computation of the PSTH grand average showed a clear increase of the deviant to standard response ratio after the SR subtraction (Fig. 6e, $\Delta f=0.37$ at $4 \mathrm{~Hz}$ ). At a population level, this trend is maintained. Figure 7a shows the scatter plots for the same pairs of neurons recorded in the awake preparation, before and after the SR subtraction (burgundy and orange, respectively). The neurons that showed low levels of SSA $(\mathrm{CSI}<0.4)$ remain mostly unaltered when the SR was subtracted, while the neurons with higher levels (CSI $\geq 0.4$ ) showed an increase of SSA (data not shown). That happened regardless of the condition, but was most clearly noticeable when the levels of CSI were consistently high (for that reason in Fig. 7 we only show 4 of the 9 conditions shown in Fig. 2 ; i.e., $\Delta f=0.1$ and $\Delta f=0.37$ at 4 and $8 \mathrm{~Hz}$ ). Interestingly, the qualitative non-significant differences found between the awake and the anesthetized preparation (Fig. 2) seem to disappear when the SR is subtracted (Fig. 7b).

\section{Discussion}

The present study demonstrates high levels of SSA in the mouse IC and, more importantly, it shows that SSA in the awake mouse is comparable to that seen in the urethaneanesthetized preparation. The SSA observed in mice is equivalent in most respects to that seen in the rat IC (Malmierca et al. 2009; Duque et al. 2012; Ayala and Malmierca 2013). Our results further reveal that urethane anesthesia does affect other response properties in the mouse IC, most notably the SR. Although previous studies have shown SSA in awake preparations in different species or brain regions, our study is the first to directly compare the effect of anesthesia in the IC. Overall, the results demonstrate that SSA is a genuine property of some IC neurons and not an artifact attributable to anesthesia, but that it is dynamically modulated by the animal's state through the neuronal SR.

A detailed examination of SSA at the population level revealed no major differences between the anesthetized and the awake conditions in the proportion of neurons showing SSA and in the overall level of SSA that the 

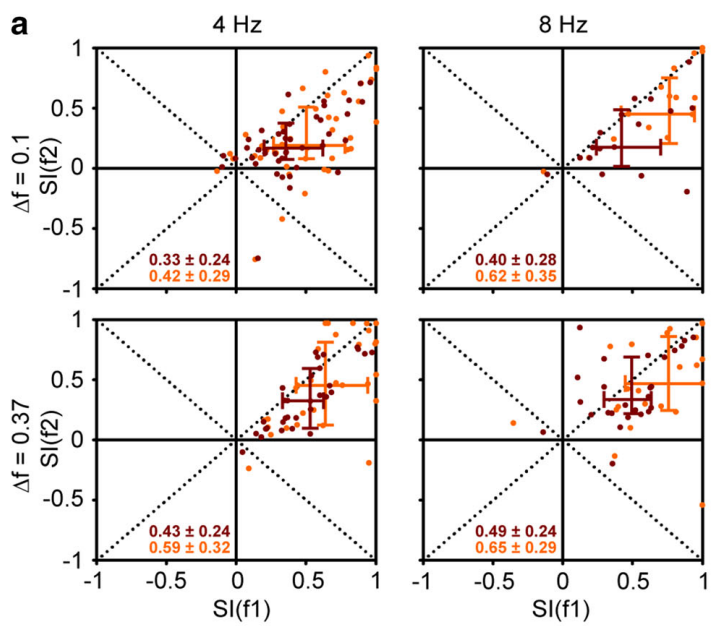

b
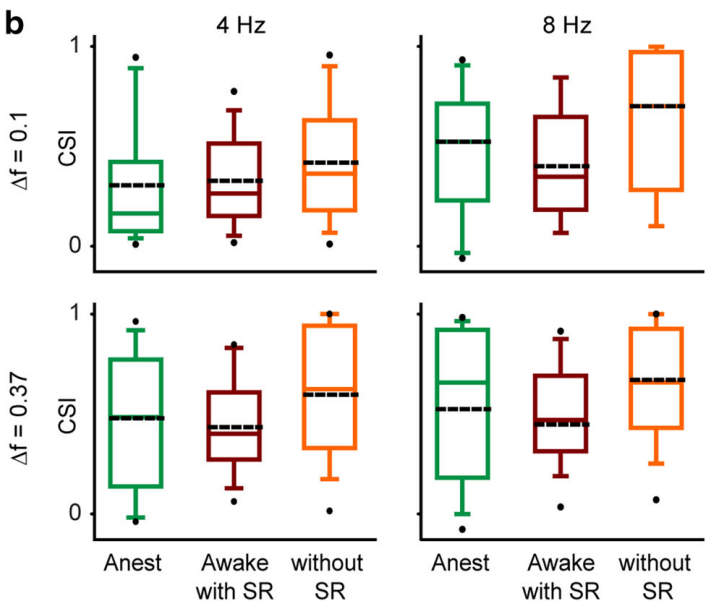

Fig. 7 Population comparison between the awake neurons before and after the subtraction of the spontaneous activity. a Scatter plots of the $\mathrm{SI}(f 1)$ versus $\mathrm{SI}(f 2)$, for different $\Delta f$ (top row 0.1 ; bottom row 0.37 ) and ISIs (left column $4 \mathrm{~Hz}$; right column: $8 \mathrm{~Hz}$ ). Pairs of frequencies obtained in the awake mouse before (burgundy dots; median value showed as a burgundy cross) and after (orange dots; median value showed as an orange cross) the subtraction of the spontaneous activity. Median CSI values for each condition are shown as insets in each scatter plot. b Box plots showing the median value of CSI (straight line) for the anesthetized (green), awake (burgundy) and awake without spontaneous activity (orange) neurons of the mice IC. Mean values are showed as a dashed line. The box plots are for the same conditions showed in Fig. 7a. The box delimits the 25th and the 75 th percentiles, whiskers indicate the 10th and 90th percentiles, and dots indicate the 5 th and 95 th percentiles

neurons exhibited (Table 1). However, we did find some differences in other properties, including a much larger mean SR in the awake preparation (Table 1) as well as an increase in the slow component $\left(A_{s}\right)$ and the steady-state $\left(A_{\text {stst }}\right)$ indices in the dynamics of adaptation (Fig. 3; Table 1). Urethane anesthesia produces a decrease of spontaneous glutamate output in rat cerebral cortex (Moroni et al. 1981) and could have a similar effect on the IC. The high rates of SR allowed us to uncover a high incidence of inhibition in the IC and revealed a large proportion of purely inhibitory response maps (iFRAs). To the best of our knowledge, iFRAs have not been previously documented in the mouse IC, either in the anesthetized or the awake preparation. Similar inhibitory receptive fields have been described in the dorsal cochlear nucleus of the unanesthetized decerebrate cat (Young and Brownell 1976), the anesthetized guinea pig (Stabler et al. 1996), the awake mouse (Roberts and Portfors 2008) and, very rarely, in the IC of the anesthetized rat (Hernandez et al. 2005). Previous studies have shown higher evoked and spontaneous firing rates in the awake rabbit IC (Kuwada et al. 1989; Chung et al. 2014). We did not find higher evoked firing rates in the awake mice, but higher SR does seem to slightly decrease the levels of SSA (Figs. 6, 7). Although, theoretically, subtraction of SR will necessarily increase SSA based on the equation for CSI (i.e., without SR numerator will remain constant while denominator will become smaller by 4 times the SR), in practice, we observed a full range of changes. As a rule, neurons showing low levels of SSA also had the lowest variations of CSI levels (median variation: 0.02) while neurons with higher levels of SSA presented higher variations (median variation: 0.10; Mann-Whitney rank sum test, $p=0.027$ ). These results are in accordance with our theoretical approximation, as we subtracted the SR according to the evaluation of the difference in the response with respect to a basal level. A recent study (Klein et al. 2014) discussed the possibility that spontaneous activity might be responsible for the low levels of SSA in the auditory cortex. The present data show that in the IC the SR has some effect on SSA sensitivity. Our data are in accord with previous elegant work that suggests a basal adaptive state due to high spontaneous activity rates (Abolafia et al. 2011). However, these potassium currents suggested by Abolafia and colleagues do not completely explain SSA (because they would affect all inputs and thus, will not be stimulus-specific). Such pre-adaptive situation will produce less adaptation to both the standard and deviant stimuli, decreasing the ratio between the deviant and the standard response, therefore reducing the levels of SSA. Indeed, since high SR in the awake behaving animals are related to a reduction of adaptation (Chung et al. 2002; Castro-Alamancos 2004), it is likely that attention during task engagement modulates both the evoked and the spontaneous firing rate (Buran et al. 2014). In this respect, we found a clear correlation between the CSI levels and the SR such that the higher the CSI level, the lower the SR (Fig. 6d). Thus, it is tempting to speculate that the attentional state might be dynamically 
increasing the SR and, consequently, affecting the levels of SSA. One plausible mechanism would be through GABAergic inhibition. We have previously demonstrated that $\mathrm{GABA}_{\mathrm{A}}$ mediated inhibition acts as a gain control system that sharpens and enhances SSA (Perez-Gonzalez et al. 2012; Duque et al. 2014) and thus, might suppress the SR to increase SSA.

Since most reports on SSA from IC neurons have been in the anesthetized rat (Pérez-González et al. 2005, 2012; Malmierca et al. 2009, Lumani and Zhang 2010; Zhao et al. 2011; Duque et al. 2012; Perez-Gonzalez and Malmierca 2012; Ayala et al. 2013), it is pertinent to compare our current results with those in the rat. Table 1 and Fig. 2 not only compare the anesthetized and the awake mouse recordings, but they also include previous data from the anesthetized rat IC. All but one of these experiments employed urethane anesthesia (Lumani and Zhang 2010, used ketamine-xylazine). Urethane has been the anesthetic agent of choice in many electrophysiological recordings, since it produces (1) minimal disruption of breathing, heart rate and reflexes (Maggi and Meli 1986), (2) small changes in the receptor system by non-selectively affecting both the excitatory and inhibitory currents (Hara and Harris 2002) and (3) little effect on the time-course and amplitude of inhibitory and excitatory responses (Sceniak and MacIver 2006). As stated before, the greater amount of inhibition observed in the awake preparation allowed us to confirm the presence of iFRAs compared with the absence of these type of response areas in anesthetized preparations (Chi square test for sampling distributions, $\chi^{2}=34.271$, $p<0.001)$. No significant differences were found between previous data obtained in rat IC and the current work in the awake and the anesthetized mouse in the level of SSA (Table 1, Kruskal-Wallis ANOVA on ranks test: $p=0.895$ ) or in the proportion of neurons showing SSA (Chi square test for sampling distributions, $\chi^{2}=0.586$, $p=0.746$ ). In fact, the levels of SSA in the awake mice are unexpectedly similar to the ones in the anesthetized animals considering that anesthetic and inter-specific differences affect other features such as latency difference, firing rate and, particularly, spontaneous activity (Table 1, Kruskal-Wallis test: $p=0.012, p=0.042, p<0.001$, respectively). For example, the latency difference is smaller for some conditions in the awake preparation compared with the anesthetized ones. We usually interpreted the delay in the response latency to the standard stimuli as a consequence of an independent input processing of the deviant and the standard frequencies. In such scenario, the latency of the response to the standard sound will be progressively delayed up to a point where the response to the repeated sound is no longer strong enough to evoke a response. Such a latency difference reduction in the awake preparation could be the result of a decrease in the general level of adaptation due to the pre-adaptive state (Abolafia et al. 2011). Moreover, the time course of adaptation shows slightly higher values in rat, but probably because the population used in that experiment presented an intentional bias towards highly adapting neurons (PerezGonzalez et al. 2012: median CSI: 0.78).

Our study is the first to compare the effect and influence of anesthesia on SSA by directly comparing awake and anesthetized responses in the IC in the same preparation. We were initially surprised by the similarity of the results we obtained in the awake and anesthetized preparations, as previous studies found SSA levels in awake experiments considerably lower than those found in anesthetized preparations. Several differences in the experimental paradigms may help to explain the discrepancies. First, some experiments were carried out at relatively high intensities (Von der Behrens et al. 2009) or employed fixed tones to assess SSA (Nir et al. 2013). Both of these factors may have influenced the level of SSA, as we have previously demonstrated that SSA is both level and frequency dependent (Duque et al. 2012). Further, the anatomical location of the recording sites may also have significantly influenced the reported values of SSA. For example, it is likely that the majority of the recordings in the IC of a recent study in an awake bat preparation (Thomas et al. 2012) were from the central nucleus, as bats possess a hypertrophied central nucleus and very small cortical regions compared to rats and mice (Zook et al. 1985). Similarly, studies in the medial geniculate body in the awake rat included a large number of units from ventral and dorsomedial geniculate neurons (Richardson et al. 2013) where SSA is weak (Antunes et al. 2010; Antunes and Malmierca 2011). Further, while the use of multi electrodes allowed for a largescale examination of SSA in monkey A1 (Fishman and Steinschneider 2012), they might also result in averaging out high SSA values. Interestingly, the current IC data from mice contrasts with a previous report on the medial geniculate body in mice (Anderson et al. 2009), as the levels of SSA found in the IC are markedly higher. Most likely, the different anesthesia regime (ketamine-medetomine), anatomical sampling and stimulation protocols used in the medial geniculate body study may explain the low levels of SSA reported there, since a degradation of the SSA sensitivity along the auditory pathway is unlikely (Perez-Gonzalez et al. 2012; Dhruv and Carandini 2014; Duque et al. 2014; Escera and Malmierca 2014). Moreover, it is generally accepted that anesthesia can have a more pronounced effect on the auditory cortex than on the IC (Maggi and Meli 1986; Sloan 1998; laboratory unpublished observations), since more indirect input pathways - as the auditory cortex - are more likely to be affected by anesthesia (Goldstein and Abeles 1975). Therefore, it would be very interesting to directly compare how SSA is in the 
auditory cortex in awake and anesthetized animals as we have done here in the IC in further experiments. Finally, it should be emphasized that the current experiments only provide an unequivocal demonstration that SSA is not generated by urethane anesthesia; the effect of other anesthetic agents remains unknown.

SSA has been associated with important sensory tasks, namely novelty detection or change detection (Pérez-González and Malmierca 2014) and its electrophysiological correlates, i.e. the mismatch negativity auditory evoked potential or the middle-latency response studied in detail in both humans (Näätänen et al. 1978; Slabu et al. 2010; Grimm et al. 2011) and small rodents using both the anesthetized (Tikhonravov et al. 2008; Astikainen et al. 2011; Shiramatsu et al. 2013) and awake (Nakamura et al. 2011; Jung et al. 2013) preparations. Recent studies have shown that the moderate urethane effects on neurotransmission seem to perfectly mimic natural sleep (Clement et al. 2008; Pagliardini et al. 2012, 2013), a state that implies a reduction of behavioral responsiveness (Rechtschaffen et al. 1966). While mismatch negativity shows some attenuation during natural sleep (Loewy et al. 1996; Ruby et al. 2008), our study is in agreement with a recent account (Nir et al. 2013) that showed that natural sleep does not affect the generation of SSA in the auditory cortex. Nevertheless, while urethane anesthesia closely mimics the alternations of forebrain rhythms found in the EEG components during sleep (Clement et al. 2008), the overall SR is dramatically affected by urethane, at least in the IC. Such a reduction of the SR could be related to a general tendency for high levels of SSA during sleep-like states. As we demonstrate, SSA is similar but certainly not identical, in the awake and anesthetized preparations. This suggests that during sleep we may need more robust resources to react to danger than in an awake state in which attention is already preactivated. Attenuation of SR during sleep could improve this essential response.

In conclusion, our study shows that while SSA is not due to urethane anesthesia, the awake preparation is different in that neurons show a much higher SR. These findings, neglected so far for technical reasons, should be considered in future studies, especially in view of recent work (Buran et al. 2014) demonstrating the importance of modulating the SR in attention and behavior. Our results also confirm the validity and the importance of studying SSA in the IC of the mouse because these results provide the baseline for future studies and open new avenues to the study genetic modifications affecting the auditory brain in transgenic mice. Moreover, because the IC acts as a computational center in the auditory midbrain that may gate thalamic activity (Winer et al. 1996; Peruzzi et al. 1997; Ito et al. 2009), the simple and old-fashioned IC as a relay center can be ruled out (Skoe et al. 2013a, b; Skoe and Kraus 2010; Malmierca et al. 2014), hence supporting the idea of the IC is a key controller of cortical activation, including cortical SSA.

Acknowledgments We are most grateful to Drs. Flora Antunes, Nell Cant, Douglas Oliver, Alan Palmer and Christine Portfors for their critical and valuable comments on a previous version of this manuscript. We also thank Mr. Javier Nieto and Dr. David PérezGonzález for their help and assistance in the analysis of the data. Financial support was provided by the Spanish MEC (BFU201343608-P), to MSM; DD held a fellowship from the Spanish MEC (BES-2010-035649). The funders had no role in study design, data collection and analysis, decision to publish, or preparation of the manuscript.

\section{References}

Abolafia JM, Vergara R, Arnold MM, Reig R, Sanchez-Vives MV (2011) Cortical auditory adaptation in the awake rat and the role of potassium currents. Cereb Cortex 21:977-990. doi:10.1093/ cercor/bhq163

Anderson LA, Christianson GB, Linden JF (2009) Stimulus-specific adaptation occurs in the auditory thalamus. J Neurosci 29:7359-7363. doi:10.1523/JNEUROSCI.0793-09.2009

Antunes FM, Malmierca MS (2011) Effect of auditory cortex deactivation on stimulus-specific adaptation in the medial geniculate body. J Neurosci 31:17306-17316. doi:10.1523/ JNEUROSCI.1915-11.2011

Antunes FM, Malmierca MS (2014) An overview of stimulus-specific adaptation in the auditory thalamus. Brain Topogr. doi:10.1007/ s10548-013-0342-6

Antunes FM, Nelken I, Covey E, Malmierca MS (2010) Stimulusspecific adaptation in the auditory thalamus of the anesthetized rat. PLoS One 5:e14071. doi:10.1371/journal.pone.0014071

Astikainen P, Stefanics G, Nokia M, Lipponen A, Cong F, Penttonen M, Ruusuvirta T (2011) Memory-based mismatch response to frequency changes in rats. PLoS One 6:e24208. doi:10.1371/ journal.pone. 0024208

Ayala YA, Malmierca MS (2013) Stimulus-specific adaptation and deviance detection in the inferior colliculus. Front Neural Circuits 6:89. doi:10.3389/fncir.2012.00089. eCollection 2012

Ayala YA, Perez-Gonzalez D, Duque D, Nelken I, Malmierca MS (2013) Frequency discrimination and stimulus deviance in the inferior colliculus and cochlear nucleus. Front Neural Circuits 6:119. doi:10.3389/fncir.2012.00119. eCollection 2012

Bryant JL, Roy S, Heck DH (2009) A technique for stereotaxic recordings of neuronal activity in awake, head-restrained mice. J Neurosci Methods 178:75-79. doi:10.1016/j.jneumeth.2008.11. 014

Buran BN, von Trapp G, Sanes DH (2014) Behaviorally gated reduction of spontaneous discharge can improve detection thresholds in auditory cortex. J Neurosci 34:4076-4081. doi:10.1523/JNEUROSCI.4825-13.2014

Castro-Alamancos MA (2004) Absence of rapid sensory adaptation in neocortex during information processing states. Neuron 41:455-464

Clement EA, Richard A, Thwaites M, Ailon J, Peters S, Dickson CT (2008) Cyclic and sleep-like spontaneous alternations of brain state under urethane anaesthesia. PLoS One 3:e2004. doi:10. 1371/journal.pone.0002004

Chandrasekaran B, Kraus N (2010) The scalp-recorded brainstem response to speech: neural origins and plasticity. Psychophysiology 47(2):236-246. doi:10.1111/j.1469-8986.2009.00928.x 
Chung S, Li X, Nelson SB (2002) Short-term depression at thalamocortical synapses contributes to rapid adaptation of cortical sensory responses in vivo. Neuron 34:437-446

Chung Y, Hancock KE, Nam SI, Delgutte B (2014) Coding of electric pulse trains presented through cochlear implants in the auditory midbrain of awake rabbit: comparison with anesthetized preparations. J Neurosci 34:218-231. doi:10.1523/JNEUROSCI. 2084-13.2014

Dhruv NT, Carandini M (2014) Cascaded effects of spatial adaptation in the early visual system. Neuron 81:529-535. doi:10.1016/j. neuron.2013.11.025

Duque D, Malmierca MS, Caspary DM (2014) Modulation of stimulus-specific adaptation by GABAA receptor activation or blockade in the medial geniculate body of the anaesthetized rat. J Physiol 592:729-743. doi:10.1113/jphysiol.2013.261941

Duque D, Perez-Gonzalez D, Ayala YA, Palmer AR, Malmierca MS (2012) Topographic distribution, frequency, and intensity dependence of stimulus-specific adaptation in the inferior colliculus of the rat. J Neurosci 32:17762-17774. doi:10.1523/JNEUROSCI. 3190-12.2012

Ehret G (1979) Quantitative analysis of nerve fibre densities in the cochlea of the house mouse (Mus musculus). J Comp Neurol 183(1):73-88. doi:10.1002/cne.901830107

Escera C, Malmierca MS (2014) The auditory novelty system: an attempt to integrate human and animal research. Psychophysiology 51:111-123. doi:10.1111/psyp.12156

Egorova M, Ehret G, Vartanian I, Esser KH (2001) Frequency response areas of neurons in the mouse inferior colliculus. I. Threshold and tuning characteristics. Exp Brain Res 140:145-161

Faure PA, Fremouw T, Casseday JH, Covey E (2003) Temporal masking reveals properties of sound-evoked inhibition in duration-tuned neurons of the inferior colliculus. J Neurosci 23:3052-3065

Fishman YI, Steinschneider M (2012) Searching for the mismatch negativity in primary auditory cortex of the awake monkey: deviance detection or stimulus specific adaptation? J Neurosci 32:15747-15758. doi:10.1523/JNEUROSCI.2835-12.2012

Fitzpatrick DC, Kuwada S, Batra R, Trahiotis C (1995) Neural responses to simple simulated echoes in the auditory brain stem of the unanesthetized rabbit. J Neurophysiol 74:2469-2486

Franklin K, Paxinos G (2007) The Mouse Brain in Stereotaxic Coordinates, 3rd edn. Academic Press, New York

Goldstein MH Jr, Abeles M (1975) Note on tonotopic organization of primary auditory cortex in the cat. Brain Res 100(1):188-191

Grimm S, Escera C, Slabu L, Costa-Faidella J (2011) Electrophysiological evidence for the hierarchical organization of auditory change detection in the human brain. Psychophysiology 48:377-384. doi:10.1111/j.1469-8986.2010.01073.x

Hara K, Harris RA (2002) The anesthetic mechanism of urethane: the effects on neurotransmitter-gated ion channels. Anesth Analg 94:313-318 (table of contents)

Hernandez O, Espinosa N, Perez-Gonzalez D, Malmierca MS (2005) The inferior colliculus of the rat: a quantitative analysis of monaural frequency response areas. Neuroscience 132:203-217

Hernández O, Rees A, Malmierca MS (2006) A GABAergic component in the commissure of the inferior colliculus in rat. Neuroreport 17:1611-1614

Holmstrom LA, Eeuwes LB, Roberts PD, Portfors CV (2010) Efficient encoding of vocalizations in the auditory midbrain. J Neurosci 30:802-819. doi:10.1523/JNEUROSCI.1964-09.2010

Ito T, Bishop DC, Oliver DL (2009) Two classes of GABAergic neurons in the inferior colliculus. $J$ Neurosci 29(44):13860-13869. doi:10.1523/JNEUROSCI.3454-09.2009

Izquierdo MA, Gutiérrez-Conde PM, Merchán MA, Malmierca MS (2008) Non-plastic reorganization of frequency coding in the inferior colliculus of the rat following noise-induced hearing loss. Neuroscience 154:355-369. doi:10.1016/j.neuroscience. 2008.01.057

Jones S, Mill R, Denham S, Klump G (2012) Stimulus-specific adaptation in the inferior colliculus of the awake gerbil in response to Markov chain tone sequences. ARO Midwinter Meeting, abstr 795, San Diego, USA

Jung F, Stephan KE, Backes H, Moran R, Gramer M, Kumagai T, Graf R, Endepols H, Tittgemeyer M (2013) Mismatch responses in the awake rat: evidence from epidural recordings of auditory cortical fields. PLoS One 8:e63203. doi:10.1371/journal.pone. 0063203

King AJ, Nelken I (2009) Unraveling the principles of auditory cortical processing: can we learn from the visual system? Nat Neurosci 12(6):698-701. doi:10.1038/nn.2308

Klein C, von der Behrens W, Gaese BH (2014) Stimulus-specific adaptation in field potentials and neuronal responses to frequency-modulated tones in the primary auditory cortex. Brain Topogr. doi:10.1007/s10548-014-0376-4

Kuwada S, Batra R, Stanford TR (1989) Monaural and binaural response properties of neurons in the inferior colliculus of the rabbit: effects of sodium pentobarbital. J Neurophysiol 61:269-282

Loewy DH, Campbell KB, Bastien C (1996) The mismatch negativity to frequency deviant stimuli during natural sleep. Electroencephalogr Clin Neurophysiol 98:493-501

Lumani A, Zhang H (2010) Responses of neurons in the rat's dorsal cortex of the inferior colliculus to monaural tone bursts. Brain Res 1351:115-129. doi:10.1016/j.brainres.2010.06.066

Maggi CA, Meli A (1986) Suitability of urethane anesthesia for physiopharmacological investigations in various systems. Part 1: general considerations. Experientia 42:109-114

Malmierca MS (2003) The structure and physiology of the rat auditory system: an overview. Int Rev Neurobiol 56:147-211

Malmierca MS, Ryugo DK (2011) Auditory system. The mouse nervous system, 1st edn. Academic Press, San Diego, pp 607-645

Malmierca MS, Hernandez O, Rees A (2005) Intercollicular commissural projections modulate neuronal responses in the inferior colliculus. Eur J Neurosci 21(10):2701-2710. doi:10.1111/j. 1460-9568.2005.04103.x

Malmierca MS, Cristaudo S, Perez-Gonzalez D, Covey E (2009) Stimulus-specific adaptation in the inferior colliculus of the anesthetized rat. J Neurosci 29:5483-5493

Malmierca MS, Izquierdo MA, Cristaudo S, Hernández O, PérezGonzález D, Covey E, Oliver DL (2008) A discontinuous tonotopic organization in the inferior colliculus of the rat. J Neurosci 28:4767-4776. doi:10.1523/JNEUROSCI.0238-08. 2008

Malmierca MS, Sanchez-Vives MV, Escera C, Bendixen A (2014) Neuronal adaptation, novelty detection and regularity encoding in audition. Front Syst Neurosci 8:111. doi:10.3389/fnsys.2014. 00111

Merrill EG, Ainsworth A (1972) Glass-coated platinum-plated tungsten microelectrodes. Med Biol Eng 10:662-672

Moroni F, Corradetti R, Casamenti F, Moneti G, Pepeu G (1981) The release of endogenous GABA and glutamate from the cerebral cortex in the rat. Naunyn-Schmiedeberg's Arch Pharmacol 316:235-239

Muniak MA, Mayko ZM, Ryugo DK, Portfors CV (2012) Preparation of an awake mouse for recording neural responses and injecting tracers. J Vis Exp. doi:10.3791/3755

Nääanen R, Gaillard AW, Mantysalo S (1978) Early selectiveattention effect on evoked potential reinterpreted. Acta Psychol (Amst) 42:313-329

Nakamura T, Michie PT, Fulham WR, Todd J, Budd TW, Schall U, Hunter M, Hodgson DM (2011) Epidural auditory event-related 
potentials in the rat to frequency and duration deviants: evidence of mismatch negativity? Front Psychol 2:367. doi:10.3389/fpsyg. 2011.00367. eCollection 2011

Nir Y, Vyazovskiy VV, Cirelli C, Banks MI, Tononi G (2013) Auditory responses and stimulus-specific adaptation in rat auditory cortex are preserved across NREM and REM sleep. Cereb Cortex. doi:10.1093/cercor/bht328

Pagliardini S, Funk GD, Dickson CT (2013) Breathing and brain state: urethane anesthesia as a model for natural sleep. Respir Physiol Neurobiol 188:324-332. doi:10.1016/j.resp.2013.05.035

Pagliardini S, Greer JJ, Funk GD, Dickson CT (2012) State-dependent modulation of breathing in urethane-anesthetized rats. J Neurosci 32:11259-11270. doi:10.1523/JNEUROSCI.0948-12.2012

Perez-Gonzalez D, Malmierca MS (2012) Variability of the time course of stimulus-specific adaptation in the inferior colliculus. Front Neural Circuits 6:107. doi:10.3389/fncir.2012.00107. eCollection 2012

Pérez-González D, Malmierca MS (2014) Adaptation in the auditory system: an overview. Front Integr Neurosci 8:19. doi:10.3389/ fnint.2014.00019

Pérez-González D, Malmierca MS, Covey E (2005) Novelty detector neurons in the mammalian auditory midbrain. Eur J Neurosci 22:2879-2885

Perez-Gonzalez D, Hernandez O, Covey E, Malmierca MS (2012) GABA(A)-mediated inhibition modulates stimulus-specific adaptation in the inferior colliculus. PLoS One 7:e34297. doi:10.1371/journal.pone.0034297 [Erratum in: PLoS One. 2012;7(8). doi:10.1371/annotation/883c90e9-2108-449f-a6522cbf25ba6456]

Peruzzi D, Bartlett E, Smith PH, Oliver DL (1997) A monosynaptic GABAergic input from the inferior colliculus to the medial geniculate body in rat. J Neurosci 17(10):3766-3777

Portfors CV, Roberts PD, Jonson K (2009) Over-representation of species-specific vocalizations in the awake mouse inferior colliculus. Neuroscience 162:486-500. doi:10.1016/j.neu roscience.2009.04.056

Portfors CV, Mayko ZM, Jonson K, Cha GF, Roberts PD (2011) Spatial organization of receptive fields in the auditory midbrain of awake mouse. Neuroscience 193:429-439. doi:10.1016/j. neuroscience.2011.07.025

Rechtschaffen A, Hauri P, Zeitlin M (1966) Auditory awakening thresholds in REM and NREM sleep stages. Percept Motor Skills 22:927-942

Rees A, Sarbaz A, Malmierca MS, Le Beau FE (1997) Regularity of firing of neurons in the inferior colliculus. J Neurophysiol 77:2945-2965

Richardson BD, Hancock KE, Caspary DM (2013) Stimulus-specific adaptation in auditory thalamus of young and aged awake rats. J Neurophysiol 110:1892-1902. doi:10.1152/jn.00403.2013

Roberts PD, Portfors CV (2008) Design principles of sensory processing in cerebellum-like structures. Early stage processing of electrosensory and auditory objects. Biol Cybern 98:491-507. doi: 10.1007/s00422-008-0217-1

Ruby P, Caclin A, Boulet S, Delpuech C, Morlet D (2008) Odd sound processing in the sleeping brain. J Cogn Neurosci 20:296-311. doi:10.1162/jocn.2008.20023

Sceniak MP, Maciver MB (2006) Cellular actions of urethane on rat visual cortical neurons in vitro. J Neurophysiol 95:3865-3874

Shiramatsu TI, Kanzaki R, Takahashi H (2013) Cortical mapping of mismatch negativity with deviance detection property in rat. PLoS One 8:e82663. doi:10.1371/journal.pone.0082663. eCollection 2012

Skoe E, Chandrasekaran B, Spitzer ER, Wong PC, Kraus N (2013a) Human brainstem plasticity: the interaction of stimulus probability and auditory learning. Neurobiol Learn Mem 109C:82-93. doi:10.1016/j.nlm.2013.11.011

Skoe E, Kraus N (2010) Auditory brain stem response to complex sounds: a tutorial. Ear Hear 31(3):302-324. doi:10.1097/AUD. Ob013e3181cdb272

Skoe E, Krizman J, Spitzer E, Kraus N (2013b) The auditory brainstem is a barometer of rapid auditory learning. Neuroscience 243:104-114. doi:10.1016/j.neuroscience.2013.03.009

Slabu L, Escera C, Grimm S, Costa-Faidella J (2010) Early change detection in humans as revealed by auditory brainstem and middle-latency evoked potentials. Eur J Neurosci 32:859-865. doi:10.1111/j.1460-9568.2010.07324.x

Sloan TB (1998) Anesthetic effects on electrophysiologic recordings. J Clin Neurophysiol: Off Publ Am Electroencephalogr Soc 15(3):217-226

Song P, Wang N, Wang H, Xie Y, Jia J, Li H (2011) Pentobarbital anesthesia alters neural responses in the precedence effect. Neurosci Lett 498:72-77. doi:10.1016/j.neulet.2011.04.066

Stabler SE, Palmer AR, Winter IM (1996) Temporal and mean rate discharge patterns of single units in the dorsal cochlear nucleus of the anesthetized guinea pig. J Neurophysiol 76:1667-1688

Stiebler I, Ehret G (1985) Inferior colliculus of the house mouse. I. A quantitative study of tonotopic organization, frequency representation, and tone-threshold distribution. J Comp Neurol 238(1):65-76. doi:10.1002/cne.902380106

Ter-Mikaelian M, Sanes DH, Semple MN (2007) Transformation of temporal properties between auditory midbrain and cortex in the awake Mongolian gerbil. J Neurosci 27:6091-6102

Thomas JM, Morse C, Kishline L, O'Brien-Lambert A, Simonton A, Miller KE, Covey E (2012) Stimulus-specific adaptation in specialized neurons in the inferior colliculus of the big brown bat, Eptesicus fuscus. Hear Res 291:34-40

Tikhonravov D, Neuvonen T, Pertovaara A, Savioja K, Ruusuvirta T, Naatanen R, Carlson S (2008) Effects of an NMDA-receptor antagonist $\mathrm{MK}-801$ on an $\mathrm{MMN}$-like response recorded in anesthetized rats. Brain Res 1203:97-102. doi:10.1016/j. brainres.2008.02.006

Tollin DJ, Populin LC, Yin TC (2004) Neural correlates of the precedence effect in the inferior colliculus of behaving cats. J Neurophysiol 92:3286-3297

Ulanovsky N, Las L, Nelken I (2003) Processing of low-probability sounds by cortical neurons. Nat Neurosci 6:391-398

von der Behrens W, Bauerle P, Kossl M, Gaese BH (2009) Correlating stimulus-specific adaptation of cortical neurons and local field potentials in the awake rat. J Neurosci 29:13837-13849. doi:10.1523/JNEUROSCI.3475-09.2009

Winer JA, Saint Marie RL, Larue DT, Oliver DL (1996) GABAergic feedforward projections from the inferior colliculus to the medial geniculate body. Proc Natl Acad Sci USA 93(15):8005-8010

Willott JF, Parham K, Hunter KP (1988) Response properties of inferior colliculus neurons in young and very old $\mathrm{CBA} / \mathrm{J}$ mice. Hear Res 37:1-14

Young ED, Brownell WE (1976) Responses to tones and noise of single cells in dorsal cochlear nucleus of unanesthetized cats. J Neurophysiol 39:282-300

Zhao L, Liu Y, Shen L, Feng L, Hong B (2011) Stimulus-specific adaptation and its dynamics in the inferior colliculus of rat. Neuroscience 181:163-174. doi:10.1016/j.neuroscience.2011.01. 060

Zook JM, Winer JA, Pollak GD, Bodenhamer RD (1985) Topology of the central nucleus of the mustache bat's inferior colliculus: correlation of single unit properties and neuronal architecture. J Comp Neurol 231(4):530-546 
Study IV: Frequency channels determines SSA

Wang X, Duque D, Malmierca MS (2014)

The width of the frequency channels determines

stimulus-specific adaptation in the inferior colliculus of the rat.

Under review. 

TITLE

The width of the frequency channels determines stimulus-specific adaptation in the inferior colliculus of the rat

\section{AUTHORS}

Xin Wang $^{1 * \ddagger}$, Daniel Duque $^{1^{*}}$ and Manuel S. Malmierca ${ }^{1,2+}$

\section{ADDRESSES}

1 Auditory Neurophysiology Unit, Laboratory for the Neurobiology of Hearing, Institute of Neuroscience of Castilla y León, University of Salamanca, Salamanca 37007, Spain.

${ }^{2}$ Department of Cell Biology and Pathology, Faculty of Medicine, University of Salamanca, Campus Miguel de Unamuno, 37007, Salamanca, Spain.

* these authors contributed equally to this work

\section{ADDITIONAL INFORMATION}

Running title: Frequency channels determines SSA

Key words: Auditory, SSA

Number of pages: 34; Number of figures: 9; Number of tables: 1

Abstract number of words: $\mathbf{2 4 5}$

Introduction number of words: $\mathbf{5 6 0}$

Discussion number of words: 1585

Section: systems/circuitry

${ }^{\dagger}$ Correspondence should be sent to:

Manuel S. Malmierca

Institute of Neuroscience of Castilla y León, University of Salamanca

C/ Pintor Fernando Gallego, 1

37007 Salamanca, Spain

msm@usal.es Phone: +34 923294500, ext. 5333 Fax: +34 923294750

‡ Current address: College of Life Sciences and Hubei Key Laboratory of Genetic Regulation and Integrative Biology, Central China Normal University, Wuhan 430079, China 


\section{Author contributions}

The experiments were performed in the Laboratory for the Neurobiology of Hearing, Institute of Neuroscience of Castilla y León, University of Salamanca, Salamanca, Spain. The contribution of each author to the following aspects of the study is as stated: (1) collection of data: X.W.; (2) conception and design of experiments: D.D., M.S.M.; (3) analysis and interpretation of data: X.W., D.D. and M.S.M.; (4) writing the paper: D.D. and M.S.M. All authors approved the final version of the manuscript.

\section{Acknowledgements}

We are most grateful to Dr. Eric Young for his critical and valuable comments on a previous version of this manuscript. We also thank Mr. Javier Nieto for his help and assistance in the analysis of the data. Financial support was provided by the Spanish MINECO (BFU2013-43608-P), to M.S.M. D.D. held a fellowship from the Spanish MEC (BES-2010-035649). X.W. held a fellowship from the National Natural Science Foundation of China (NNSFC-31000493). The funders had no role in study design, data collection and analysis, decision to publish, or preparation of the manuscript. 


\section{ABSTRACT}

For years, electrophysiological, psychophysical and electroencephalographic

3 studies have tried to disentangle the neuronal basis for intensity coding and intensity

4 deviant detection. Psychophysical forward masking experiments have repeatedly

5 shown how a higher intensity sound masks the subsequent low intensity sound, but

6 electroencephalographic mismatch negativity experiments have proved that pre-

7 attentive deviant detection can be elicited with low intensity deviants sounds. Here we

8 did extracellular single-unit recording in the inferior colliculus (IC) of the anesthetized

9 rat to test if there is stimulus-specific adaptation (SSA) for intensity deviants. We used

10 the oddball paradigm to evaluate SSA for frequency, intensity and double deviants for

11 frequency and intensity. Thus, if we considered two sounds of the same frequency

12 where the low intensity sound presented a low probability of appearance, two scenarios

13 could arise: 1) neurons adjust to stimulus statistics by changing the dynamic range to

14 the high intensity sound or 2) SSA exists for intensity sounds and the neuron presents

15 an enhanced response for the low intensity deviant sound. Our results demonstrate that

16 there is no SSA for purely intensity deviant sounds in the IC, but the across-adaptation

17 data analysis show that SSA can be found for double deviants whenever the high

18 intensity standard present a frequency that is outside the frequency channels that code

19 for the deviant sound. Moreover, those frequency channels broaden at higher intensities

20 and are clearly narrower for neurons that show high levels of SSA, strongly suggesting

21 that the frequency-channel theory is explaining SSA in the IC. 
While neuronal systems seem to follow an efficient coding strategy to properly

24 respond the most common inputs (Wark et al., 2007), repetition in the brain usually

25 implies adaptive processes (Grill-Spector et al., 2006). The range of intensities and

26 frequencies that an animal can perceive is enormous and environmental changes need

27 to be assessed rapidly and accurately. The auditory system needs to adjust its response

28 to the stimulus statistics (Dean et al., 2005; Watkins and Barbour 2008; Wen et al.,

29 2009; Dahmen et al., 2010; Rabinowitz et al., 2011), while the response to the less

30 common sounds (deviants) cannot be neglected and usually present an enriched

31 response (stimulus-specific adaptation; SSA: Ulanovsky et al., 2003; Malmierca et al.,

32 2009). This issue has been recently discussed by two recent studies (Herrmann et al.,

33 2014; Simpson et al., 2014).

34 Most studies on SSA have been realized with frequency deviant sounds 35 (Nelken 2014), while the investigation about dynamic range adaptation has been 36 basically performed with intensity distributions (Dean et al., 2005; 2008; Watkins and

37 Barbour 2008; 2011; Wen et al., 2009; 2012). Beyond the frequency SSA, some

38 investigators try to evoke such process by a plethora of features including intensity 39 (Ulanovsky et al., 2003; Reches and Gutfreund, 2008; Farley et al., 2010), interaural 40 differences (Reches and Gutfreund, 2008; Xu et al., 2014) and duration (Farley et al., 41 2010), but the existing data for intensity SSA is controversial and inconclusive. Those 42 studies disagree regarding the response to a low intensity deviant sound embedded in a 43 background of loud sounds. This issue is important for two reasons. It is well known 44 that 1) a high intensity sound mask the subsequent low intensity sound (forward masking/suppression; Calford and Sample, 1995; Brosch and Schreiner, 1997) and 2)

46 SSA is assumed to lie upstream the generation of mismatch negativity (MMN; Escera 47 and Malmierca, 2013) and such auditory evoked potential can be elicited with low 48 intensity deviants sounds (Jacobsen et al., 2003; Althen et al., 2011). Intriguingly, the 49 adjustment of the neuronal response to sound intensity statistics will reduce the 50 response to low intensity sounds if the most common sound has a higher intensity (Dean 51 et al., 2005). But, at least in the auditory cortex, some neurons are able to preserve a 52 delicate sensitivity to low intensity sounds (Watkins and Barbour, 2008). Therefore, 53 SSA for low intensity deviant sounds could be evoked, even when the high intensity 54 sound had the same frequency than the low intensity one. 
55 We recorded extracellular single-unit IC responses in the anesthetized rat to 56 test if there is SSA for intensity deviants. We calculate the frequency response area 57 (FRA) for each neuron and tested the oddball paradigm for a fixed low intensity deviant 58 sound but repeatedly varying both the frequency and the intensity of the high intensity 59 standard sound. We also used the novel rapid adaptation paradigm to characterize the 60 shape and width of the frequency channels that code for the low intensity deviant sound. 61 Our results demonstrate that there is no SSA for purely intensity deviant sounds in the 62 IC, and the analysis of the across-adaptation elicited by the double deviants for 63 frequency and intensity show that SSA can be generated if and when the high intensity 64 standard is outside the frequency channels that code for the low intensity deviant sound. 65 This experiments reinforced the idea that SSA is a feature dependent on input-specific 66 adaptation mechanisms. 
68 Surgical procedures. Experiments were performed on 37 adult pigmented female rats

69 (Rattus norvergicus, Long-Evans) with body weights between 150 and $260 \mathrm{~g}$. All

70 experimental procedures were carried out at the University of Salamanca with the

71 approval of, and using methods conforming to the standards of, the University of

72 Salamanca Animal Care Committee. Anesthesia was induced (1.5 g/kg, i.p., 20\%

73 solution) and maintained ( $0.5 \mathrm{~g} / \mathrm{kg}$, i.p. given as needed) with urethane. Urethane was

74 chosen as an anesthetic because its effects on multiple aspects of neural activity,

75 including inhibition and spontaneous firing, are known to be less than those of

76 barbiturates and other anesthetic drugs (Hara and Harris, 2002). The respiration was

77 maintained artificially (SAR-830/P Ventilator) monitoring the end-tidal $\mathrm{CO}_{2}$ level

78 (CapStar-100). For this purpose, the trachea was cannulated and atropine sulfate $(0.05$

$79 \mathrm{mg} / \mathrm{kg}$, s.c.) was administered to reduce bronchial secretions. Details of surgical

80 procedures have been described previously (Pérez-González et al., 2005; Malmierca et

81 al., 2009). Body temperature was maintained at $38 \pm 1^{\circ} \mathrm{C}$ by means of a heating blanket.

82 The animal was placed in a stereotaxic frame in which the ear bars were replaced by

83 hollow speculae that accommodated a sound delivery system, inside a sound-sealed

84 room. An incision was made in the scalp along the midline, and the skin was reflected

85 laterally before a craniotomy was performed to expose the cerebral cortex overlaying

86 the left IC.

87 Electrophysiological recording. Extracellular single unit responses were recorded 88 using a tungsten electrode (1-2 M 2 , Merrill and Ainsworth, 1972) lowered through the 89 cortex by means of a piezoelectric microdrive (Burleigh 6000 ULN). Neuron location

90 in the IC was based on stereotaxic coordinates, physiological criteria of tonotopicity 91 and response properties (Malmierca et al., 2003; Hernandez et al., 2005) and confirmed

92 histologically afterwards. Acoustic stimuli were delivered through a sealed acoustic 93 system using two electrostatic loudspeakers (TDT-EC1: Tucker Davis Technologies)

94 driven by two TDT-ED1 modules. The stimuli were presented contralaterally to the

95 recording side; search stimuli were pure tones or noise bursts monaurally delivered 96 under computer control using TDT System II hardware and custom software (Faure et 97 al., 2003; Pérez-González et al., 2005; Malmierca et al., 2009). The output of the system 98 at each ear was calibrated in situ using a 1/4" condenser microphone (model 4136, Brüel $99 \&$ Kjær) and a dynamic signal analyzer (Photon+, Brüel \& Kjær). The maximum output 
of the TDT system was flat from 0.3 to $5 \mathrm{kHz}(\sim 100 \pm 7 \mathrm{~dB}$ SPL) and from 5 to $40 \mathrm{kHz}$ ( $\sim 90 \pm 5 \mathrm{~dB}$ SPL). The highest frequency produced by this system was limited to 40

$102 \mathrm{kHz}$. The second and third harmonic components in the signal were $\geq 40 \mathrm{~dB}$ below the

103 level of the fundamental frequency at the highest output level (Malmierca et al., 2009).

104 Action potentials were recorded with a BIOAMP amplifier (TDT), the 10x output of 105 which was further amplified and bandpass-filtered (TDT PC1; $f_{\mathrm{c}}, 500 \mathrm{~Hz}$ and $3 \mathrm{kHz}$ ) 106 before passing through a spike discriminator (TDT SD1). Spike times were logged with 107 a resolution of $\approx 150 \mu$ s on a computer by feeding the output of the spike discriminator 108 into an event timer (TDT ET1) synchronized to a timing generator (TDT TG6). 109 Stimulus generation and on-line data visualization were controlled with custom 110 software. Spike times were displayed as dot rasters sorted by the acoustic parameter 111 varied during testing.

112 From each isolated neuron, the approximate frequency tuning was audiovisually 113 determined by presenting pure tones lasting $75 \mathrm{~ms}$ with a $5 \mathrm{~ms}$ rise/fall time (Hernandez 114 et al., 2005). We obtained the monaural frequency response area (FRA), the 115 combination of frequencies and intensities capable of evoking a response, as an 116 estimation of the neuronal receptive field. For that, we presented multiple combinations 117 of frequency and intensity using an automated procedure with 5 stimulus repetitions at 118 each frequency (from 0.5 to $40 \mathrm{kHz}$, in 25 logarithmic steps, presented randomly) and 119 intensity (10 dB steps, presented from lower to higher intensities). The spike counts 120 evoked at each combination of frequency and intensity were plotted using MATLAB ${ }$.

121 Stimulus presentation paradigms. The representation of the FRA allowed us to choose 122 different pairs of tones within the auditory field of the neuron. First of all, we set a pair 123 of frequencies $\left(f_{1}\right.$ and $\left.f_{2}\right)$ that elicited a similar firing rate at 10-20 dB above the best 124 frequency threshold. Then, stimuli were presented in an oddball paradigm similar to 125 that used to record mismatch negativity responses in human (Näätänen, 1992) and 126 animal studies (e.g., Ulanovsky et al., 2003; Malmierca et al., 2009). Briefly, a train of 127400 stimuli containing both frequencies $f_{l}$ and $f_{2}$ was presented under the oddball 128 paradigm: one frequency $\left(f_{l}\right)$ was presented as the standard stimuli while, interspersed 129 randomly among the standards, the deviant stimuli were presented at the second 130 frequency $\left(f_{2}\right)$. After obtaining one data set, the relative probabilities of the two stimuli 131 were reversed, with $f_{2}$ as the standard and $f_{1}$ as the deviant. At the regular frequency 132 deviant oddball paradigm used in this manuscript, the frequency contrast remained 
constant at $\Delta \mathrm{f}=0.10(0.141$ octaves $)$; where $\Delta \mathrm{f}=\left(f_{2}-f_{l}\right) /\left(f_{2} \times f_{1}\right)^{1 / 2}$. The stimuli were always presented at a repetition rate of $4 \mathrm{~Hz}$ (inter-stimulus interval, ISI $=250 \mathrm{~ms}$ ) and the probability of appearance of the deviant stimulus was fixed at $10 \%$. This condition has previously shown to evoke high neuronal levels of SSA in the IC (Malmierca et al., 2009; Duque et al., 2012). Thus, we used it to calculate an overall level of frequencydeviant SSA of each neuron. In order to have a more reliable analysis of the adaptation phenomenon, we fixed one of the frequencies used before (generally $f_{l}$ ) and calculated the response of that frequency in a deviant alone protocol, where we tested an oddball paradigm but the standard stimuli is replaced by silence. Under that circumstance, the response to the deviant stimuli is the maximum possible for a given frequency because

143 it is not affected by any kind of adaptation.

144 Besides the calculation of the level of SSA for frequency deviants, we used the oddball 145 paradigm to characterize how different frequencies at different intensities could affect 146 the response to a low intensity deviant sound (Figure 1A). For this reason, keeping the 147 deviant frequency fixed, we repeated the oddball paradigm but varied the intensity 148 contrast $(\Delta \mathrm{i}=10 \mathrm{~dB}, \Delta \mathrm{i}=20-30 \mathrm{~dB}$ and $\Delta \mathrm{i}=40-50 \mathrm{~dB})$, the frequency contrast $(\Delta \mathrm{f}=0$, $149 \Delta \mathrm{f}=0.04[0.057$ octaves $], \Delta \mathrm{f}=0.10[0.141$ octaves $]$ and $\Delta \mathrm{f}=0.37[0.526$ octaves $]$ ) or both. 150 As before, after obtaining each data set the relative probabilities of the two stimuli were 151 reversed. The analysis of the response to the deviant sound allowed us to obtain a map 152 of the different standard sounds that affect the low intensity deviant sound. Figure 1B 153 shows three different examples of the usage of the oddball paradigm to this purpose: 1) 154 pure frequency deviant oddball paradigm $(\Delta \mathrm{f}=0.1$, orange hexagon $), 2)$ pure intensity 155 deviant oddball paradigm $(\Delta \mathrm{i}=10$, violet square $)$ and 3$)$ double deviant oddball $156 \operatorname{paradigm}(\Delta \mathrm{f}=0.1$ and $\Delta \mathrm{i}=10$, black diamond). Hereinafter, when we speak of intensity 157 and double deviant protocols, deviant and probe (p) will refer to the frequency fixed at 158 the low intensity, while standard and conditioner (c) will refer to the frequency used at 159 high intensities. When probing for SSA at different frequency- and intensity contrasts, 160 we started to collect the data from the smaller intensity contrast $(\Delta \mathrm{i}=10)$ and we used, 161 at least, two different frequency contrasts. Then, we tried to cover all the possible range 162 of intensity contrasts at the same frequency contrasts used before. A complete protocol 163 in a neuron lasted for $\sim 90 \mathrm{~min}$ and allowed us to see the effect of 13 different 164 frequencies /intensities over the probe sound (Figure 1A). In order to simplify the 165 analysis of the data and to reduce the time of the experimental protocol, we decided to 
166 always pick conditioner frequencies higher than the probe sound. This decision was 167 taken because SSA levels are more evident at the high frequency range (Duque et al., 168 2012).
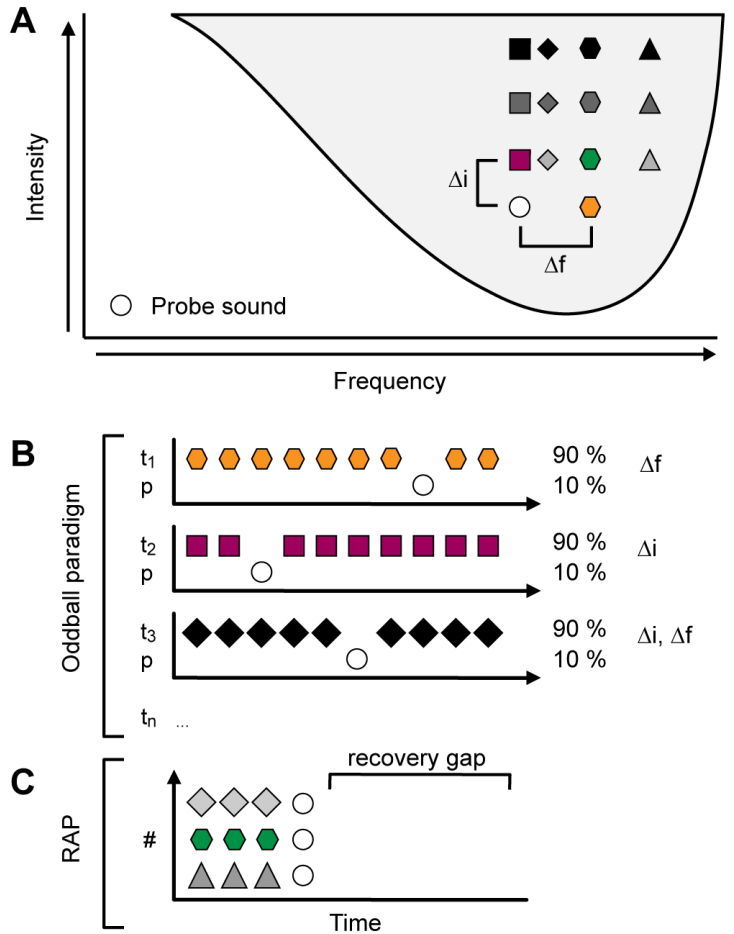

Figure 1. Experimental design. A. Schematic FRA showing the stimulation protocol of the experiments. A low intensity deviant pure tone (white circle) is fixed at the neuronal best frequency 10-20 $\mathrm{dB}$ over threshold. Different conditioner sounds (squares, diamonds, hexagons and triangles) at different frequency- $(\Delta f)$ and intensity contrasts $(\Delta i)$ are used to check the across adaptation to the low intensity sound. B. Oddball paradigm. Four hundred pure tone sequences with a deviant (10\% prob.) and a standard sound $(90 \%$ prob.) were presented. The ISI was kept constant al $250 \mathrm{~ms}$. Several different pairs of frequencies

arise considering the standard sound used: frequency deviant (orange hexagon); intensity deviant (violet square); double deviant (black diamond)... Low intensity deviant sound responses are analyzed to check for across adaptation. C. Rapid adaptation paradigm (RAP). Two thousand ms sequences with 4 tones ( 3 repeated high intensity standard sounds and the low intensity deviant sound; ISI=250 ms) and 1000 $\mathrm{ms}$ silence period (recovery gap) were presented. The whole range of frequencies and intensities used for computing the FRA is used in the RAP protocol. Reduced low intensity deviant sound responses after a determined high intensity standard sound are assumed to be due across adaptation.

169 Subsequently, with the aim of complete the previous oddball paradigm data with the

170 effect of low frequency conditioners over the probe sound, we established a novel rapid 171 adaptation paradigm (RAP, Figure 1C). The RAP merged the concepts of two tones 172 suppression experiments (e.g., Nelson et al., 2009) with the protocol to generate a FRA 173 (see above). A sequence is generated with 1) a random tone at a determined frequency 174 and intensity (conditioner, c) repeated three times before 2) a fixed sound (probe, p) is 
175 presented. The stimuli were presented at a rate of $4 \mathrm{~Hz}(\mathrm{ISI}=250 \mathrm{~ms})$ and a recovery

176 gap of $1000 \mathrm{~ms}$ is established after the probe sound, generating a $2000 \mathrm{~ms}$ sequence

177 with 4 sounds and a $1000 \mathrm{~ms}$ silence period (Figure 1C. $c c c p$ ). If the conditioner

178 frequency were related to the probe sound, the adaptation observed during the three-

179 conditioner repeated tones would also adapt the probe sound. If both tones are

180 unrelated, the response to the probe sound will be as is obtained when the probe is

181 presented alone, unaffected by the adaptation observed during the repetition of the

182 three-conditioner tones. Similar to the FRA, we presented 4 sequence repetitions at

183 multiple frequencies (25 logarithmic steps, presented randomly) and intensities (10 dB

184 steps, presented from lower to higher intensities), covering the previously generated

185 FRA. The firing rate of the probe sound-related to the conditioner sound- was then

186 plotted in MATLAB $\AA$. The graph obtained showed an area of frequencies and

187 intensities within the FRA with suppressed responses. The bandwidth of the frequency

188 channel was taken to be the frequencies where the response to the probe sound was less

189 than (1-criterion) * baseline response. The baseline response was the mean response to

190 the probe tone when it was preceded by conditioner tones at the lowest intensity; the

191 criterion values was 0.4 (Scholes et al, 2011). Bandwidths at 10 and $30 \mathrm{~dB}$ relative to

192 the best frequency threshold (reTh) were calculated. The ratio between the bandwidths

193 of the frequency channel and the FRA was also computed to extract the relative width

194 of the frequency channel.

195 Data analysis. Dot raster plots are used to illustrate the responses obtained to the 196 oddball paradigm, plotting individual spikes (red dots indicate responses to the deviant; 197 blue dots to the standard, and green to the deviant in a deviant alone protocol). Stimulus 198 presentations are marked along the vertical axis. The responses to the standard and 199 deviant stimuli were expressed as spikes per stimulus in a peri-stimulus time histogram 200 (PSTH), to account for the different number of presentations in each condition. The 201 amount of SSA was quantified in different ways. First, we calculated the common SSA 202 index (CSI) and the frequency-specific index $\left(\mathrm{SI}_{f l}\right)$ from the firing rate elicited in the 203 oddball paradigm. They were defined as CSI $=\left[d\left(f_{1}\right)+\mathrm{d}\left(f_{2}\right)-s\left(f_{1}\right)-\mathrm{s}\left(f_{2}\right)\right] /\left[d\left(f_{1}\right)+d\left(f_{2}\right)\right.$ $\left.204+s\left(f_{1}\right)+s\left(f_{2}\right)\right]$, where $\mathrm{d}(\mathrm{f})$ and $\mathrm{s}(\mathrm{f})$ are responses to each frequency $f_{1}$ or $f_{2}$ when they 205 were the deviant (d) or standard (s) stimulus and as $\mathrm{SI}_{f l}=\left[\mathrm{d}\left(f_{l}\right)-\mathrm{s}\left(f_{l}\right)\right] /\left[\mathrm{d}\left(f_{l}\right)+\mathrm{s}\left(f_{l}\right)\right]$, 206 defined for the fixed frequency $(f 1)$. The values of these indices range from -1 to +1 , 207 being positive if the response to the deviant stimulus is greater. Both indexes are well 
208 defined and have been used in previous studies, proving to be useful when the firing 209 rate of the both frequencies is similar and when used for computing SSA for frequency 210 deviants (e.g., Ulanovsky et al., 2003; Malmierca et al., 2009). We also used the 211 normalized index of adaptation (NIA) defined for deviant as $\mathrm{NIA}_{\mathrm{dev}}=\mathrm{d}\left(f_{1}\right) / \mathrm{d}\left(f_{\text {l-alone }}\right)$

212 and for standard as $\mathrm{NIA}_{\mathrm{std}}=\mathrm{s}\left(f_{1}\right) / \mathrm{d}\left(f_{\text {l-alone }}\right)$. We do not use a correction for spontaneous 213 rate because the values are usually negligible in the urethane-anesthetized rat and mice 214 (Duque et al., 2012; Duque and Malmierca, 2014). The NIA works with the assumption 215 that the response to the sound in the deviant alone protocol is the maximum possible 216 for a given frequency because is not affected by any kind of adaptation. In the NIA, 217 responses to the standard or deviant sound are divided by the response in the deviant 218 alone protocol, reflecting the extent to which the response to the standard or the deviant 219 is reduced compared to the computed maximum response. NIA range from 0 to 1 , being 2201 if the response to the sound is maximal (i.e., not adapted) and 0 if the response to the 221 sound is totally suppressed. A Wilcoxon rank paired t-test comparing the NIA values 222 for the standard $\left(\mathrm{NIA}_{\text {std }}\right)$ and the deviant $\left(\mathrm{NIA}_{\text {dev }}\right)$ at the same condition allows for 223 computing SSA.

224 Statistical tests were performed using non-parametric tests. For comparing data from 225 different groups, we used Mann-Whitney rank tests. For comparisons between the same 226 data at different conditions, we used Wilcoxon rank paired t-tests. Multiple 227 comparisons were realized with the Kruskal-Wallis test and the differences were 228 confirmed with the Dunn's post-hoc analysis. All the statistical tests were considered 229 significant when $p \leq 0.05$. Different statistical tests were noted in the paper. The analysis 230 and figures were done using Sigmaplot 11 (Systat Software) and MATLAB® 231 (MathWorks). 
We recorded single unit responses from 132 well-isolated neurons in the IC of

234 the rat, determined the basic temporal and spectral response properties of each neuron

235 and chose a pair of frequencies within the FRA to evaluate SSA for frequency deviants

236 under an oddball paradigm. Then, in order to test whether or not genuine SSA exists

237 for intensity deviants, we fixed one of the frequencies used for the frequency deviant

238 protocol and tested again the oddball paradigm but in this case for sounds that only

239 differed by intensity. Finally, we also checked how responses to high intensity sounds

240 affect the level of SSA of a low intensity tone. In the following, first we describe SSA

241 responses of IC neurons for frequency and intensity deviants and then we will detail

242 how the responses to low intensity sounds are modified by high intensity sounds.

\section{SSA for frequency deviants}

244 The common SSA index (CSI) was used to quantify the degree of neuronal 245 adaptation in an oddball paradigm with a frequency contrast $(\Delta \mathrm{f})$ of 0.1 and a repetition 246 rate of $4 \mathrm{~Hz}(n=117)$, a condition that previous studies demonstrated to evoke high 247 levels of SSA (Malmierca et al., 2009). CSI levels in this condition range from -0.09 to 2480.99 with an average of $0.49 \pm 0.34$ (mean \pm S.D.) and confirm our previous data 249 (Malmierca et al., 2009, Duque et al., 2012; Ayala et al., 2013). A CSI cut-off value of $250+0.18$ was defined as significant SSA based on previous data (e.g. Antunes et al., 2010). 251 Using this criterion, 81 neurons $(69 \%)$ in our sample showed significant SSA, while 252 the remaining $36(31 \%)$ did not. We also quantified the degree of SSA using the 253 frequency-specific SSA index (SI). The scatter plot in figure 2A shows the SI values 254 for each frequency used in the oddball paradigm $\left(\mathrm{SI}_{f l} \mathrm{Vs}\right.$. $\left.\mathrm{SI}_{f 2}\right)$. As expected (Malmierca 255 et al., 2009; Duque et al., 2012, 2014; Ayala et al., 2013), the majority of values are 256 located in the upper 'right' quadrant, and therefore they show significant SSA.

\section{SSA for intensity deviants} the neuron again using the oddball paradigm. In this case the second sound had the same frequency $(\Delta \mathrm{f}=0)$ but different intensity $(\Delta \mathrm{i}=10 \mathrm{~dB})$. As a control, we also tested

261 the oddball paradigm while varying both the frequency and the intensity, establishing 262 a double deviant protocol $(\Delta \mathrm{f}=0.1 ; \Delta \mathrm{i}=10 \mathrm{~dB}$, Figure $1 \mathrm{~A})$. Hereinafter, when we speak 
263 of intensity and double deviant protocols, $f 1$ and probe (p) will refer the frequency fixed

264 at the low intensity. To facilitate comparisons, the colors of the conditions in the scatter

265 plots shown in Figure 2 are the same as in Figure 1: the open white circle is the fixed

266 probe frequency $(f 1, \mathrm{p})$ and the 3 different colors represent the 3 different standard

267 frequencies (conditioner, c) at the 3 different oddball paradigm protocols. Figure 2B

268 shows the scatter plot for the SI values in the double deviant condition, i.e., when we

269 varied frequency and intensity in concert ( $n=97$; the low intensity probe sound [ $f 1]$ is

270 presented in the $x$-axis). The levels of CSI recorded in this condition range from -0.04

271 to 0.99 , with a mean value of $0.51 \pm 0.33$ (mean \pm S.D.). The distribution of the dots in

272 Figure 2A and 2B is almost identical, as the majority of values are located in the upper

273 'right' quadrant, demonstrating unambiguously the presence of genuine SSA, meaning

274 adaptation for both frequencies as standards. Nevertheless, a few SI values for the low

275 intensity sound $\left(\mathrm{SI}_{f 1}: 6\right.$ cases, $\left.6 \%\right)$ lie at $\mathrm{SI}=-1$, meaning that there is no response at all

276 for the low intensity deviant sound.

277 By contrast, Figure 2C shows the scatter plot for the SI values when we tested 278 an oddball paradigm with two sounds of the same frequency that differed in intensity 279 only ( $\Delta \mathrm{i}=10 \mathrm{~dB}, n=117$; the low intensity sound [fl] is presented in the $x$-axis). The CSI 280 values range from -0.04 to 0.92 with a mean CSI value of $0.35 \pm 0.29$ (mean \pm S.D.). 281 Since the CSI values for the intensity deviant condition were lower than the values 282 obtained before for the frequency deviant and the control condition, we run a Kruskall283 Wallis ANOVA on Ranks test to check if there were some differences between the 284 conditions $(\mathrm{H}=16.70 ; \mathrm{p}<0.001)$. Dunn's method post hoc test confirmed that the CSI 285 values in the intensity deviant condition were smaller than in the frequency and the 286 double deviant condition $(\mathrm{Q}=3.72$ and $\mathrm{Q}=3.26$, respectively; $\mathrm{p}<0.01$ in both cases). 287 Furthermore, a simple visual inspection of the values in Figure 2A and 2B show a 288 different distribution to that at Figure $2 \mathrm{C}$, because of the SI values obtained in the 289 oddball paradigm for the low intensity sounds ( $\left.\mathrm{SI}_{f l}\right)$. Indeed, a majority of the values 290 (95 out of 117 neurons analyzed; 81.2\%) were found in the upper 'left' quadrant and 291 had a negative $\mathrm{SI}_{f I}$ value. Moreover, 44 values $(37.8 \%)$ are unresponsive to low 292 intensity sounds, show a $-1 \mathrm{SI}_{f l}$ and lay on the left $y$-axis. Only 4 neurons $(3.4 \%)$ 293 presented a $\mathrm{SI}_{f l}$ value larger than 0.18 (the cut-off value used for significant SSA), 294 although a detailed analysis of the $\mathrm{SI}_{f I}$ values show that they were not different from 0 295 and, therefore, we considered the values outliers (bootstrap over 1000 randomizations). 

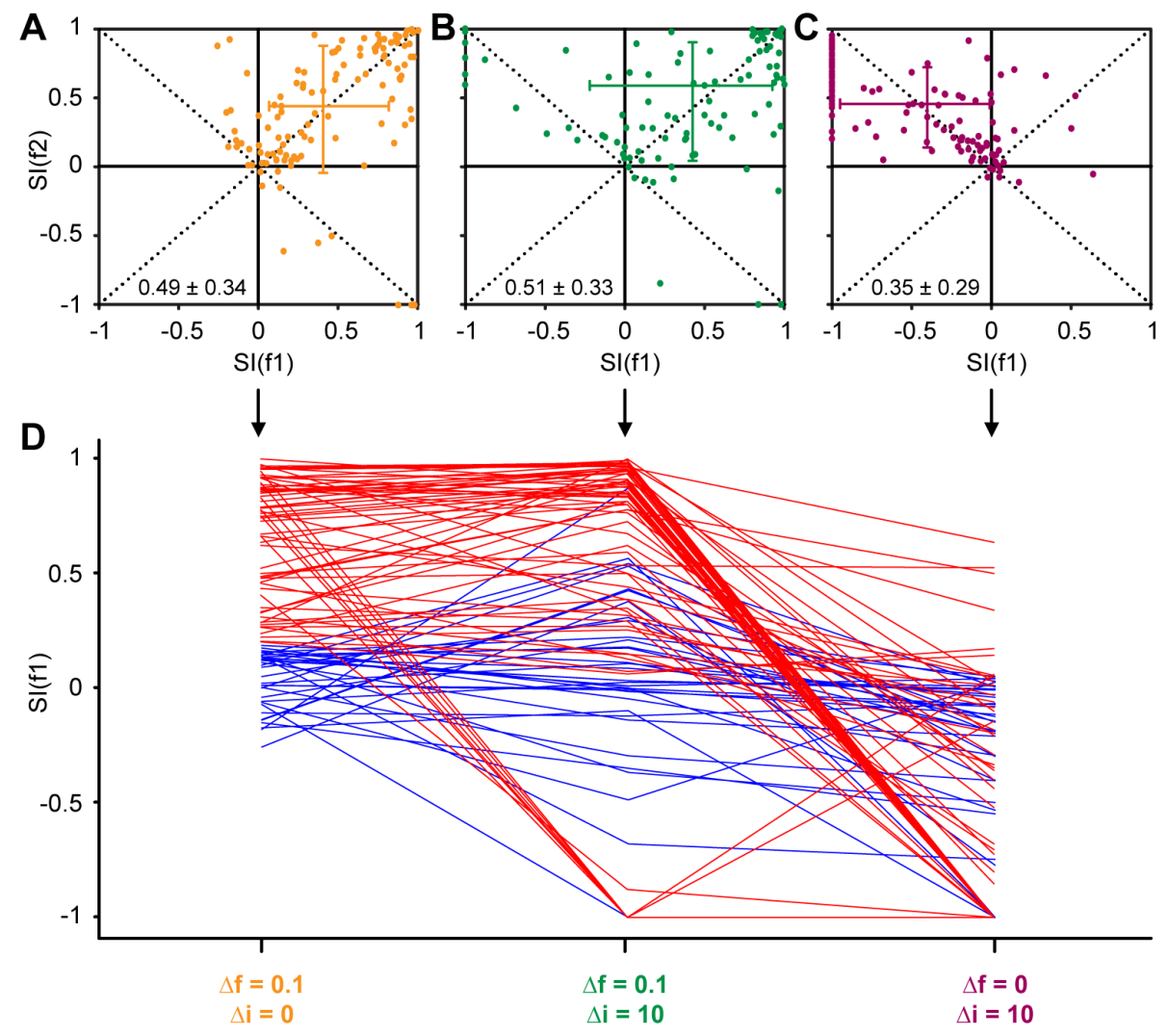

Figure 2. IC neurons do not show pure intensity deviant SSA. A. Scatter plot of the $\operatorname{SI}(f 1)$ versus $\operatorname{SI}(f 2)$ for the frequency deviant pairs of frequencies analyzed at a $\Delta f=0.1$. The cross indicates the median and the $25^{\text {th }}-75^{\text {th }}$ interquartile range for each axis. Each neuron was tested using different combinations of parameters and may be represented in additional panels. Median CSI value is shown at the bottom of the plot. B. Scatter plot of the $\operatorname{SI}(f 1)$ versus $\operatorname{SI}(f 2)$ for the double deviant condition (mixed frequency and intensity deviant pairs of frequencies) analyzed at a $\Delta f=0.1$ and $\Delta i=10 . \operatorname{SI}(f 1)$ : Low intensity probe SI. SI $(f 2)$ : High intensity conditioner SI.C. Scatter plot of the $\operatorname{SI}(f 1)$ versus $\operatorname{SI}(f 2)$ for the intensity deviant pairs of frequencies analyzed at a $\Delta i=10$. $\operatorname{SI}(f 1)$ : Low intensity probe SI. SI $(f 2)$ : High intensity conditioner SI. D. Changes in $\operatorname{SI}(f 1)$ values for each neuron at the three previous conditions: pure frequency deviant (left column), double deviant (middle column) and pure intensity deviant (right column). The values are sorted for neurons with low- (blue lines) and high SSA (red lines) for frequency deviants. Note the drop in intensity SSA levels for neurons with good sensitivity for frequency SSA. Neurons with low frequency SSA sensitivity present also low levels for intensity SSA. 

than the CSI, the results indicate in reality an apparent SSA for intensity deviant sounds. At first sight, we can observe two clearly differentiated populations. The first one, which showed $\mathrm{SI}_{f l}$ values for frequency deviants larger than +0.18 (red lines, significant SSA levels), generally presented similar values in the frequency deviant condition (Figure 2D, left column) and the double deviant condition (Figure 2D, middle column), but a big $\mathrm{SI}_{f l}$ drop when we test the oddball paradigm for the intensity deviant condition (Figure 2D, right column). As before, in several cases the $\mathrm{SI}_{f l}$ values are -1, indicating that there is no response to the low intensity sound. The second population showed $\mathrm{SI}_{f 1}$ values smaller than +0.18 (Figure 2D, blue lines) and had neither SSA for frequency nor for intensity deviants, with $\mathrm{SI}_{f l}$ values generally close to 0 in the three different conditions. The above indicates that the 'classic CSI' metric is not appropriate to evaluate intensity deviants because it is clearly biased by the reverse condition in the oddball paradigm, where the deviant sound presents a consistent response when it has a higher intensity than the standard sound. Figure 3 shows a typical example illustrating this effect. For the dot rasters (Figure 3B-E) we only highlight the responses to the low intensity sound colored (Figure 3A, fl, white empty circle) in the three different conditions shown before: frequency-, double- and intensity deviant. Figure 3B shows the response to $f 1$ in a deviant alone protocol (green dots and lines), where the response should not be affected by adaptation and, therefore, to be maximum (see Methods).

The evaluation of the CSI for the frequency- (Figure 3C) and the double deviant condition (Figure 3D) undoubtedly embodies genuine SSA, as compared to the $\mathrm{SI}_{f l}$ values. But when evaluating purely intensity deviants (Figure 3E) CSI fails to represent SSA, giving values comparable to the other conditions because of the bias

321 due to the $\mathrm{SI}_{f 2}$ value obtained in the reverse high intensity deviant condition (grey dots).

322 A closer inspection to the dot rasters in Figure 3E allows to see the vanishing of the 323 response to the low intensity deviant (Figure 3E, no red dots in the bottom scatter plot) 324 when the standard sound is louder, while the response to the high intensity deviant 325 sound is extremely reliable because the standard has a lower intensity and it is not 326 affecting the response to the high intensity deviant (Figure 3E, grey dots). 

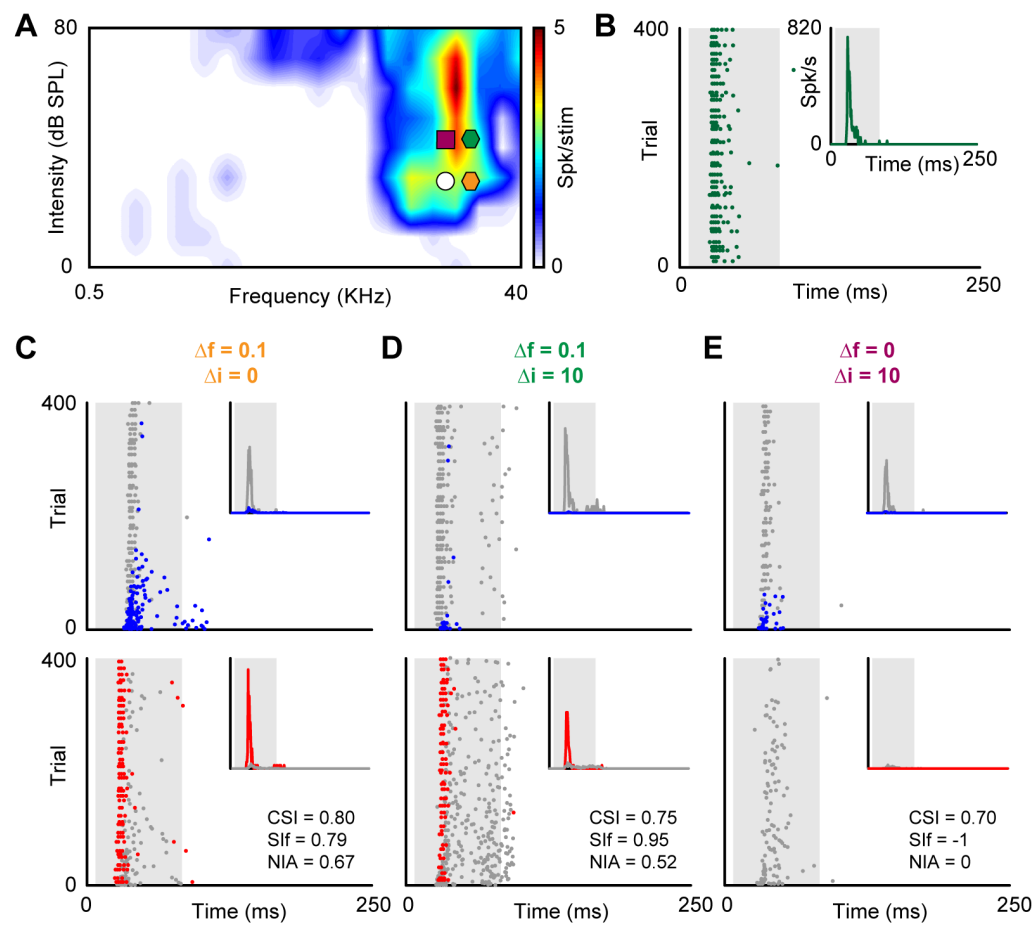

Figure 3. CSI misrepresent intensity SSA. A. FRA of an IC neuron. A low intensity sound ( $f 1$, white circle) and three different frequencies ( $\Delta f=0.1$ : orange hexagon; $\Delta f=0.1$ at $\Delta i=10$ : green hexagon; $\Delta i=10$ : violet square) are represented over the FRA. B. Dot raster plot illustrating responses of the low intensity sound in the deviant alone protocol. C-E. Below the FRA, dot raster plots are illustrated for the oddball paradigm with 3 three different frequencies establishing: C. a frequency deviant oddball paradigm, $\Delta f=0.1$ : orange hexagon in $3 \mathrm{~A}$. D. a double deviant oddball paradigm, $\Delta f=0.1$ at $\Delta i=10$ : green hexagon in $3 \mathrm{~A}$ and $\mathbf{E}$. a intensity deviant oddball paradigm, $\Delta i=10$ : violet square in $3 \mathrm{~A}$. In the top row the response to the low intensity sound as standard (90\%) are represented in blue. In the bottom row -the reverse condition- responses to the low intensity sound as deviant (10\%) are represented in red. Insets represent the PSTHs for the low intensity sound as deviant (red) or standard (blue). Responses to the other frequencies are plotted in grey but are not analyzed. Shaded backgrounds indicate the duration of the stimulus. CSI, $\operatorname{SI}(f I)$ and NIA values obtained in each condition are shown as insets in the bottom row. Observe that the CSI value obtained do not reflect the response observed in the intensity deviant condition (red responses in $\mathbf{E}$ ).

Next, we wonder if the frequency specific SI is a better index for studying SSA

328 at the intensity domain. In some cases, when no response is present for the low intensity 329 deviant (Figure $3 \mathrm{E}$ ), $\mathrm{SI}_{f l}$ works properly to evaluate intensity SSA. In other cases, a 330 minimal response also biased the SSA levels observed by $\mathrm{SI}_{f l}$. Figure 4 illustrates an 
331 example where the CSI fails to reflect the neural SSA in the intensity deviant case and $332 \mathrm{SI}_{f 1}$ also fails to do it in this case (Figure 4E). The consistent, although minimal, 333 response to the low intensity deviant sound (red dots in the bottom Figure 4E) results 334 in an exceptionally high level of $\mathrm{SI}_{f l}$ that reflect the responses observed in the dot rasters 335 for the frequency- and the double deviant condition inaccurately (Figure 4C-D). Thus, 336 in order to define and use an indicator that represents more objectively the adaptation 337 in the intensity domain, we defined the normalized index of adaptation (NIA, see 338 Methods). A simple comparison between the NIA values for the standard (NIA std $_{1}$ ) and 339 the deviant sounds $\left(\mathrm{NIA}_{\mathrm{dev}}\right)$ at the same condition not only allows for a consistent SSA 340 index, but also highlight the effect of high intensity sounds on the adaptation of the low 341 intensity ones (Figure 3E and 4E).
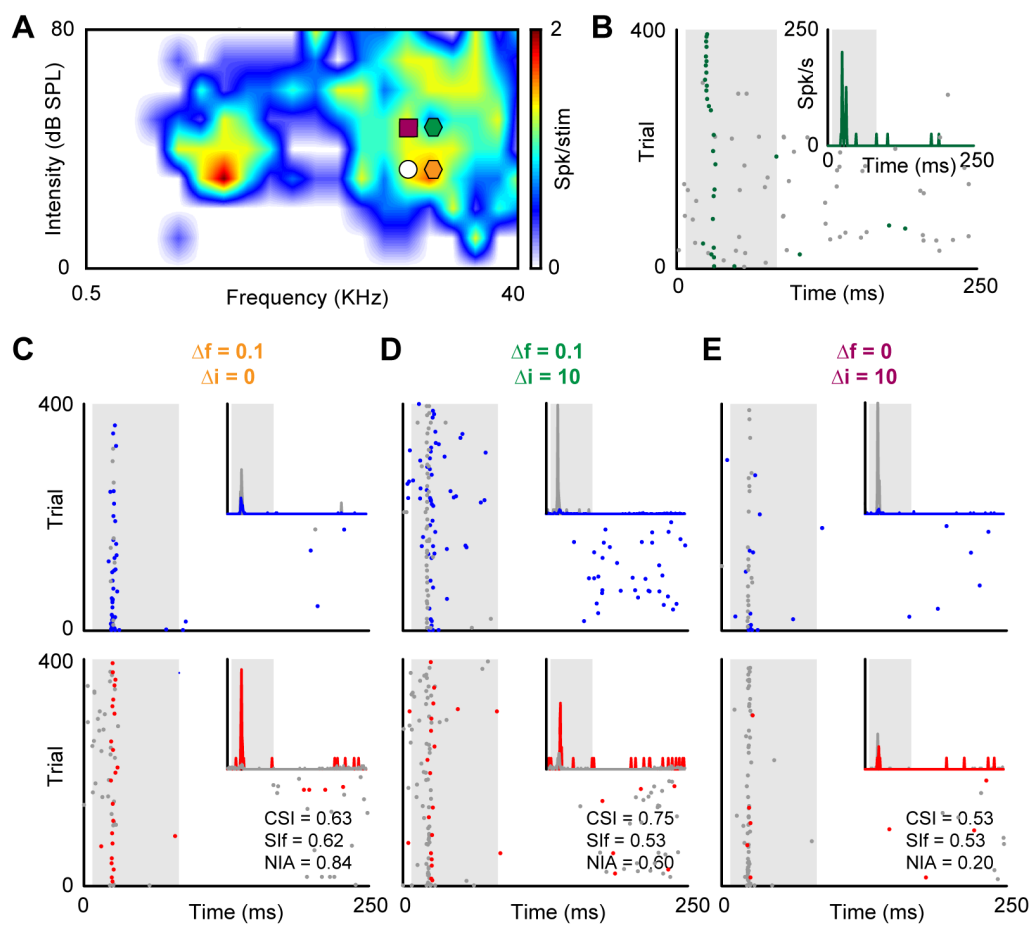

Figure 4. SI $(f t)$ misrepresent intensity SSA. Same conventions as in Figure 3. A. FRA of an IC neuron. B. Deviant alone responses for low intensity sound (fl, white circle). C. Frequency deviant responses for the low intensity sound $(\Delta f=0.1$ : orange hexagon in 4A). D. Double deviant responses for the low intensity sound ( $\Delta f=0.1$ at $\Delta i=10$ : green hexagon in $4 \mathrm{~A})$. E. Intensity deviant responses for the low intensity sound $(\Delta i=10$ : violet square in $4 \mathrm{~A})$. Note that the $\left.\operatorname{SI}_{f l}\right)$ value obtained do not reflect the response observed in the intensity deviant condition (red responses in $\mathbf{E}$ ). 
Next, we aimed to gain an understanding on how different frequencies (for now on: conditioners, c) at different intensities affect the adaptation of the low intensity sound. We used the oddball paradigm fixing one frequency ( $f 1$, for now on: probe, $p$ ) and varying the frequency contrast $(\Delta \mathrm{f}=0, \Delta \mathrm{f}=0.04, \Delta \mathrm{f}=0.10$ and $\Delta \mathrm{f}=0.37)$ and the

347 intensity contrasts $(\Delta \mathrm{i}=10, \Delta \mathrm{i}=20-30$ and $\Delta \mathrm{i}=40-50 \mathrm{~dB})$.

\section{A}
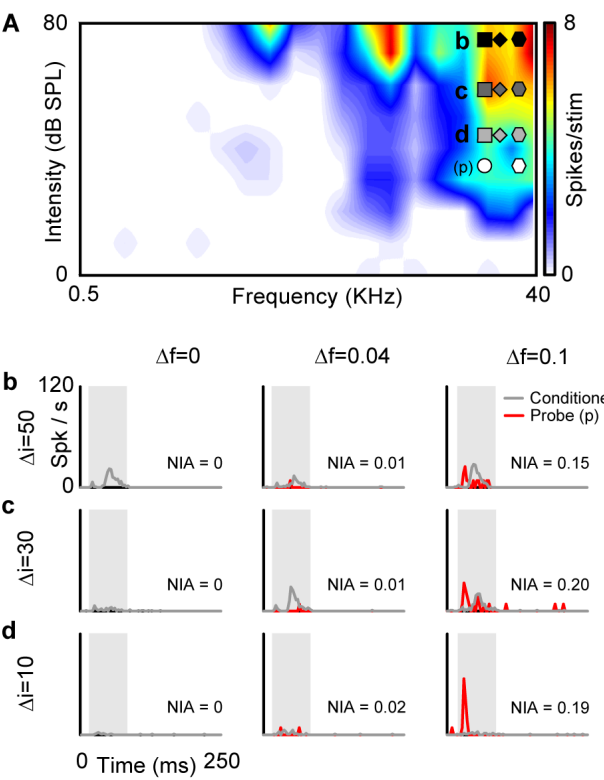$$
\text { E }
$$
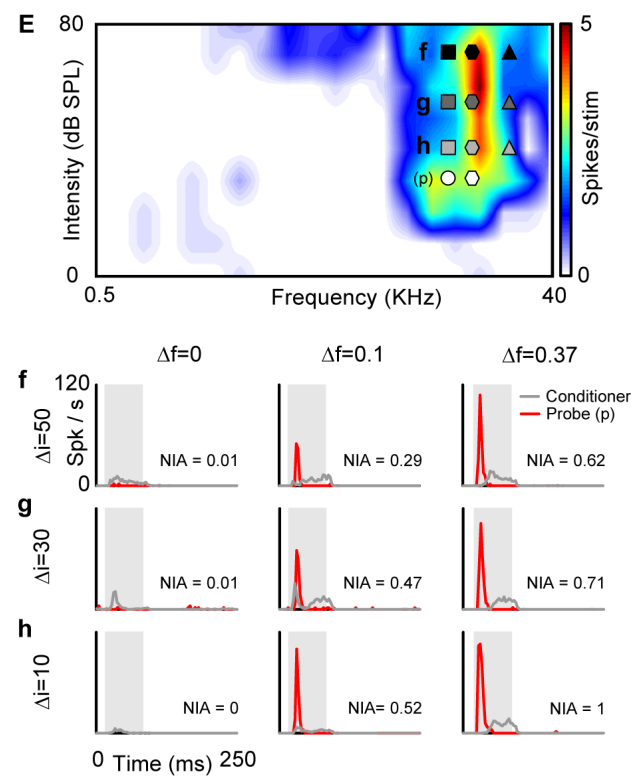

Figure 5. Two neuronal examples of intensity deviant SSA. A. FRA of an IC neuron. Probe sound (white circle, $p$ ) and nine different conditioner sounds covering the high frequency range of the FRA are represented over the FRA. The conditioner sounds were used at 3 frequency contrasts $(\Delta f=0, \Delta f=0.04$ and $\Delta f=0.10)$ with 3 intensity contrasts: b. $\Delta i=50 \mathrm{~dB}$. c. $\Delta i=30 \mathrm{~dB}$. d. $\Delta i=10 \mathrm{~dB}$. b-d. Below the FRA, PSTHs are illustrated for the probe response in the oddball paradigm with the nine different conditioner sounds. E. FRA of another IC neuron. Same conventions as in A. Oddball paradigm was performed at 3 frequency contrasts $(\Delta f=0$, $\Delta f=0.1$ and $\Delta f=0.37$ ) with 3 different intensity contrasts: $\mathbf{f}$. $\Delta i=50 \mathrm{~dB}$. g. $\Delta i=30 \mathrm{~dB}$. h. $\Delta i=10$ dB. f-h. PSTHs show the probe responses with the different conditioner sounds. Intensity deviant SSA can only be evoked if the high intensity conditioner sound differs in frequency from the probe sound. 
Figure 5 shows examples of two typical neurons. In both cases we observed 349 the lack of response to the low intensity sound as deviant when the conditioner sound 350 is the same frequency at a higher intensity (Figure $5 \mathrm{~b}-\mathrm{d}$ and $5 \mathrm{f}-\mathrm{h}$, left column, NIA $\approx 0$ ). 351 In general, at low frequency contrasts we observed the same trend (Figure 5b-d, $352 \Delta \mathrm{f}=0.04$ : middle column, NIA $\approx 0$ ), but the responses to the low intensity deviant sounds 353 usually resulted in larger NIA values at higher frequency contrasts (Figure 5f-h, middle 354 and right column, $\Delta \mathrm{f}=0.1$ and $\Delta \mathrm{f}=0.37$ respectively). When the intensity contrast is 355 larger $(\Delta \mathrm{i}=40-50 \mathrm{~dB}$, Figure $5 \mathrm{~b}$ and $5 \mathrm{f})$, the NIA levels usually decreased compared 356 with the NIA levels observed at low intensity contrasts. This findings suggests that the 357 frequency channel that codes the response for the low intensity sound gets broader as 358 sounds are louder, giving the possibility to high intensity sounds at large frequency 359 contrasts to affect the adaptation of the low intensity sound. In order to check if this notion is true, we divided the data in two groups: neurons with significant SSA at the regular frequency-deviant oddball condition 362 (Figure 6A and 6B; $\mathrm{CSI} \geq 0.18$ ) and neurons that lack SSA at the same condition (Figure $3636 \mathrm{C}$ and $6 \mathrm{D} ; \mathrm{CSI}<0.18)$. For both populations we analyzed 1) the SSA levels by 364 comparing the NIA values for the standard and the deviant sounds (Figure 6A and 6C) and 2) the latency difference between the response to the standard and that of the deviant sound (Figure 6B and 6D). When we analyzed the neurons with high frequency-

367 SSA levels, we observed -as expected- that the NIA $\mathrm{A}_{\mathrm{dev}}$ value in that condition was 368 significantly higher level than the NIA std $_{\text {(Figure 6A, first column; NIA }}$ std: blue median, 369 NIA $_{\text {dev }}$ : red median; Wilcoxon paired t-test, $Z=7.9, p<0.001$, to simplify the chart NIA $A_{\text {std }}$ 370 levels at other conditions are not shown). When we analyzed the $\mathrm{NIA}_{\operatorname{dev}}$ at a $\Delta \mathrm{f}=0$, the 371 levels are statistically different than the NIA std at the three $\Delta \mathrm{i}$, but in this condition the 372 response to the standard is always larger than the response to the deviant (Wilcoxon 373 paired t-test, low $\Delta \mathrm{i} Z=-5.2$, mid $\Delta \mathrm{i} Z=-5.0$ and large $\Delta \mathrm{i} Z=-2.7, \mathrm{p}<0.001$ in the three 374 cases). This result implies that the response to a high intensity tone clearly adapts (and 375 sometimes totally suppresses) the response to the same tone at a low intensity. If we 376 slightly change the frequency of the high intensity conditioner $(\Delta \mathrm{f}=0.04)$, the responses 377 to the low intensity deviant sound were greatly reduced, but they did not present 378 significant differences with the response to the low intensity standard response 379 (Wilcoxon paired t-test, $\mathrm{p}>0.1$ in the three cases). By contrast, at a $\Delta \mathrm{f}=0.1$ the neurons 380 recovered the differential responsiveness observed in the frequency deviant oddball 

$\Delta \mathrm{i}=4.9, \mathrm{p}<0.001 \mathrm{in}$ the three cases). This trend was maintained and even enhanced at a $\Delta \mathrm{f}=0.37$ (Wilcoxon paired t-test, low $\Delta \mathrm{i} \mathrm{Z}=4.6$, mid $\Delta \mathrm{i} \mathrm{Z}=4.1$ and large $\Delta \mathrm{i} Z=2.9$, $\mathrm{p}<0.001$ in the three conditions).
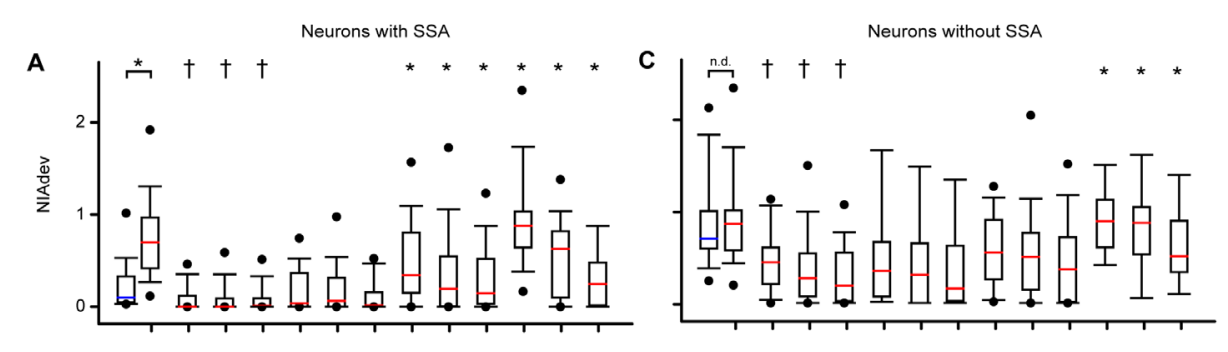

B

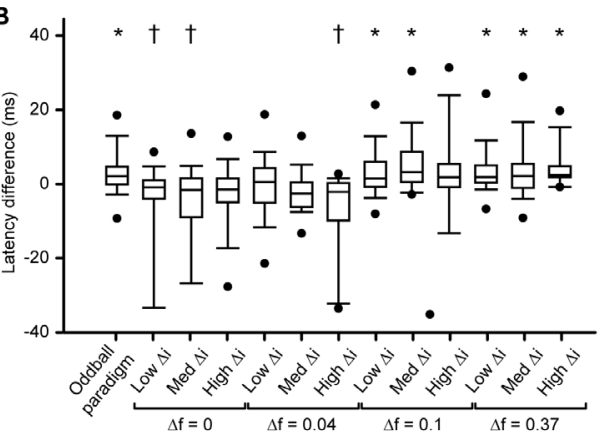

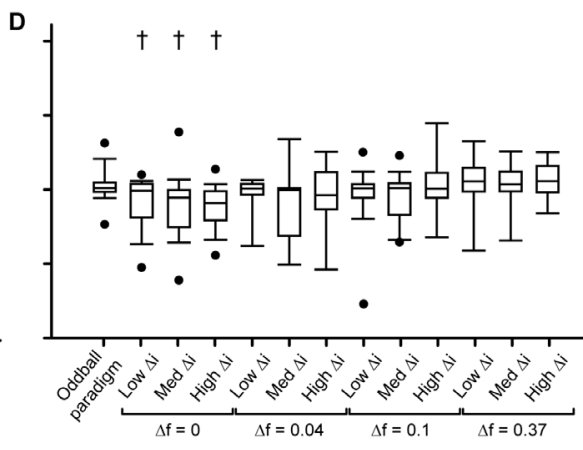

Figure 6. Frequency channels are narrow in neurons with frequency deviant SSA.

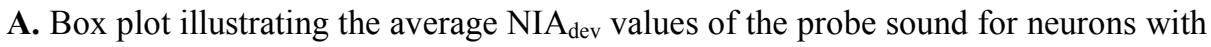
frequency deviant SSA. Different conditioners are presented at different frequency $(\Delta f=0, \Delta f=0.04, \Delta f=0.1$ and $\Delta f=0.37)$ and intensity contrasts $(\Delta i=10, \Delta i=20-30$ and $\Delta i=40-50)$. NIA std values are not plotted to simplify the plot. Asterisks $(*)$ show statistical differences $\left(\right.$ NIA $_{\text {dev }}>$ NIA $\left._{\text {std }}\right)$. Crosses $(\dagger)$ show significant differences in the other direction $\left(\mathrm{NIA}_{\operatorname{dev}}<\mathrm{NIA}_{\text {std }}\right)$. Higher responses to the low intensity deviant probe sound can be obtained when $\Delta f \geq 0.1$. B. Box plot illustrating the latency difference of the probe sound (standard - deviant) at the same conditions presented in A. The changes in latency to the probe sound mimic the changes in the NIA $_{\text {dev }}$ level. C. Box plot illustrating the average NIA $_{\text {dev }}$ values of the probe sound for neurons without frequency deviant SSA. Same conventions as in A. D. Box plot illustrating the latency difference of the probe sound (standard - deviant) at the same conditions presented in $\mathbf{C}$. Note that higher responses to the low intensity deviant probe sound can only be obtained when $\Delta f \geq 0.37$. The frequency channel that codes for the probe sounds seem to be wider in the neurons without frequency deviant SSA. 
Next, we analyzed the latency difference (Figure 6B), as a difference in latency between the standard and the deviant responses is a sign of a differential input processing of the sounds. As usual, the latency difference for the frequency deviant oddball paradigm was positive, being the latency for the standard response larger than the latency for the same sound as deviant (one sample Wilcoxon test, $\mathrm{t}=3.3, \mathrm{p}=0.001$ ). When we analyzed the latency data at a $\Delta \mathrm{f}=0$ the resultant latency difference is negative regardless of the $\Delta \mathrm{i}$, being the latency for the deviant response larger than the latency for the standard sound (one sample Wilcoxon test; low $\Delta \mathrm{i} t=-2.3$, mid $\Delta \mathrm{i} Z=-2.1$ and large $\Delta \mathrm{i} Z=-1.9 ; \mathrm{p}=0.02, \mathrm{p}=0.04$ and $\mathrm{p}=0.06$, respectively). Note that, to avoid data bias the latency difference was not calculated if the neuron showed no response to the low intensity deviant sound, but the data shows that the processing of the high intensity sound is producing a delay in the response to the low intensity sound. A similar trend was observed again at a $\Delta \mathrm{f}=0.04$ but, similarly to what we saw with the firing rate adaptation, if the high intensity sound was placed outside the theoretical frequency channel $(\Delta \mathrm{f}=0.1$ or $\Delta \mathrm{f}=0.37)$, the processing of both sounds was again independent, and the latency difference recovered the positive values observed in the frequency deviant oddball paradigm (e.g. at $\Delta \mathrm{f}=0.37$ : one sample Wilcoxon test; low $\Delta \mathrm{i} \mathrm{t}=2.6$, mid $\Delta \mathrm{i}$ $\mathrm{Z}=2.1$ and large $\Delta \mathrm{i} \mathrm{Z}=3.4 ; \mathrm{p}=0.01, \mathrm{p}=0.05$ and $\mathrm{p}=0.003$, respectively).

When we analyzed the data for the neurons with non-significant SSA $(\mathrm{CSI}<0.18)$ the trend noted for the SSA neurons was preserved, although some important differences emerged. First of all, as expected, the overall adaptation is greatly reduced compared with the neurons with significant SSA (Figure 6A-C). But, as for the

407 neurons with significant SSA, the NIA dev and NIA std levels at a $\Delta \mathrm{f}=0$ are different at the 408 three $\Delta \mathrm{i}$, presenting always a response to the standard tone higher than the response to 409 the deviant tone (Wilcoxon paired t-test, low $\Delta \mathrm{i} Z=-3.9$, mid $\Delta \mathrm{i} Z=-4.3$ and large $\Delta \mathrm{i} Z=-$ $4103.2, \mathrm{p} \leq 0.001$ in the three cases). However, the main difference was related to the 411 frequency contrast and the recovery of the deviant response to the levels observed in 412 the frequency deviant oddball paradigm: non-significant SSA neurons did not show 413 differences in the NIA levels between the responses to the same tone as deviant or 414 standard at either $\Delta \mathrm{f}=0.04$ or $\Delta \mathrm{f}=0.1$ (Wilcoxon paired t-test; $\mathrm{p}>0.2$ in all the cases, data 415 not shown) and the response to the deviant sound was only higher than the response to 416 the standard tone at a $\Delta \mathrm{f}=0.37$ (Wilcoxon paired t-test, low $\Delta \mathrm{i} Z=3.0$, mid $\Delta \mathrm{i} Z=2.0$ and 417 large $\Delta \mathrm{i} Z=2.0, \mathrm{p}<0.05$ in the three conditions). The above implies that the neurons 
418 lacking SSA possess: 1) a broader frequency channel than SSA neurons and 2) less 419 ability to adapt to sounds in general. This notion is supported by the latency data 420 analysis. As for the SSA neurons, the analysis of the latency data at a $\Delta \mathrm{f}=0$ resulted in 421 a negative latency difference regardless of the $\Delta \mathrm{i}$, being the latency for the deviant 422 response larger than the latency for the standard sound (one sample Wilcoxon test; low $423 \Delta \mathrm{i} t=-3.7$, mid $\Delta \mathrm{i} Z=-4.4$ and large $\Delta \mathrm{i} Z=-4.7 ; \mathrm{p}<0.05$ in the three cases). Again, the 424 processing of the high intensity sound affects the processing of the low intensity sound. 425 Surprisingly, the latency difference never recovered the positive values observed in the 426 regular frequency deviant oddball paradigm (one sample Wilcoxon test; $p>0.1$ in all the 427 cases at $\Delta \mathrm{f}=0.04, \Delta \mathrm{f}=0.1$ and $\Delta \mathrm{f}=0.37$ ). Thus, although the response to the high 428 intensity sound at a large frequency contrast $(\Delta \mathrm{f}=0.37)$ did not adapt the low intensity 429 sound (Figure 6C), the lack of latency difference between the standard and the deviant 430 sounds imply a certain degree of across-frequency adaptation (Figure 6D).

431 To evaluate the across-frequency adaptation from high- to low intensities, we 432 analyzed the temporal dynamics of adaptation of the standard sound at three different 433 conditions (Figure $7 \mathrm{~A})$ : with frequency- $(\Delta \mathrm{f}=0.1$; orange), double- $(\Delta \mathrm{f}=0.1, \Delta \mathrm{i}=10$; 434 green $)$ and intensity deviant sounds $(\Delta \mathrm{i}=10$; burgundy). Then, we fitted the responses 435 with a double exponential function (Figure 7B) defined as $f(t)=A_{\text {stst }}+A_{r}$. $436 e^{-t / \tau(r)}+A_{s} \cdot e^{-t / \tau(s)}($ e.g. Pérez-González et al., 2012). The response probability to 437 the standard stimulus is rapidly reduced after the first stimulus trials in the three cases, 438 but the speed of the decay is faster if the deviant sound is presented at higher intensities 439 (Figure 7A and Table 1, $\left.\tau(\mathrm{r})_{\text {freq. dev. }}=7.86 ; \tau(\mathrm{r})_{\text {double dev. }}=0.85 ; \tau(\mathrm{r})_{\text {int. dev. }}=0.78\right)$. If a high 440 intensity sound is embedded within a stream of low intensity sounds, the neuron favors 441 the response of the high intensity sound and adapt the low intensity sound, if and when 442 the high intensity conditioner is within the frequency channel of the probe sound. Note 443 that the asymptote of the curve $\left(A_{\text {stst }}\right)$ is similar in the three cases (Table 1), 444 demonstrating a common plateau at the end of the adaptation process. In other words, 445 high intensity sounds increase the speed of adaptation, but do not alter the degree of 446 adaptation. 

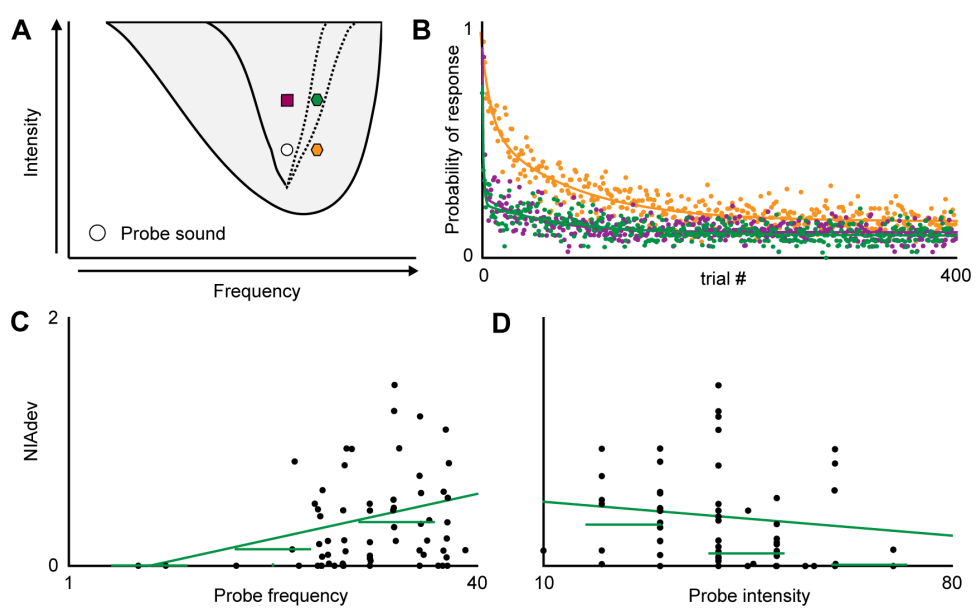

Figure 7. Frequency channel properties. A. Schematic FRA showing a probe sound (white circle) and the three conditioner sounds (orange hexagon: $\Delta f=0.1$, green hexagon: $\Delta f=0.1$ at $\Delta i=30$ and violet square: $\Delta i=30$ ) used to compute the time course of adaptation at different conditions. B. Probability of response to the standard stimulus at the three conditions stated in $\mathbf{A}$. The higher intensity conditioner allows for a rapid adaptation regardless of the frequency of the conditioner. C. Dispersion chart of the $\mathrm{NIA}_{\text {dev }}$ values against the probe frequency. The higher the frequency, the narrower the frequency channel. C. Dispersion chart of the NIA $_{\text {dev }}$ values versus the probe intensity. The higher the intensity, the wider the frequency channel.

Table 1. Double exponential coefficients at different conditions (mean $\pm 95 \%$ c.i.). Superimposition with the $95 \%$ c.i. in the control condition indicates that there are no significant differences between the groups. Asterisk (*) shows statistical differences.

\begin{tabular}{|c|c|c|c|c|c|}
\hline \multirow{2}{*}{ Condition $\left(\mathbf{r}^{2}\right)$} & \multicolumn{2}{|c|}{ Fast component } & \multicolumn{2}{|c|}{ Slow component } & \multirow{2}{*}{$\begin{array}{c}\text { Std-state } \\
\left(A_{\text {stst }}\right)\end{array}$} \\
\hline & Speed $\tau(r)$ & Decay $A_{r}$ & Speed $\tau(\mathbf{s})$ & Decay $A_{s}$ & \\
\hline $\begin{array}{l}\text { Frequency dev. } \\
(0.84)\end{array}$ & $\begin{array}{l}7.8 \\
(3.9-12.2)\end{array}$ & $\begin{array}{l}0.3 \\
(0.2-0.4)\end{array}$ & $\begin{array}{l}74.0 \\
(59.4-88.5)\end{array}$ & $\begin{array}{l}0.4 \\
(0.3-0.4)\end{array}$ & $\begin{array}{l}0.14 \\
(0.13-0.15)\end{array}$ \\
\hline $\begin{array}{l}\text { Double dev. } \\
(0.58)\end{array}$ & $\begin{array}{l}0.9 * \\
(0.5-1.2)\end{array}$ & $\begin{array}{l}1.4 * \\
(0.7-2.2)\end{array}$ & $\begin{array}{l}80.9 \\
(58.1-103)\end{array}$ & $\begin{array}{l}0.1 * \\
(0.2-0.3)\end{array}$ & $\begin{array}{l}0.09 * \\
(0.08-0.10)\end{array}$ \\
\hline $\begin{array}{l}\text { Intensity dev. } \\
(0.66)\end{array}$ & $\begin{array}{l}0.8 * \\
(0.5-1.1)\end{array}$ & $\begin{array}{l}2.0 * \\
(1.0-3.0)\end{array}$ & $\begin{array}{l}45.6 \\
(33.1-58.1)\end{array}$ & $\begin{array}{l}0.2 * \\
(0.1-0.2)\end{array}$ & $\begin{array}{l}0.10 * \\
(0.09-0.11)\end{array}$ \\
\hline
\end{tabular}

447 Non-monotonic neurons also produce adaptation through high intensity sounds

Next, we also tested if non-monotonic IC neurons with SSA are able to

449 maintain their responsiveness to low intensity sounds regardless of the intensity of the

450 conditioner tone. In order to do that, SSA neurons were classified using the 451 monotonicity index (MI: de la Rocha et al., 2008) and divided into monotonic 
$452 \quad(\mathrm{MI} \geq 0.75)$ and non-monotonic neurons $(\mathrm{MI}<0.75)$. If non-monotonic $\mathrm{IC}$ neurons 453 maintain responsiveness to low intensity sounds, the overall NIA level for the responses

454 to the low intensity deviant in the non-monotonic neurons would be larger than the NIA 455 for the same condition in the monotonic ones. We tested this possibility at all the 456 frequency $(\Delta \mathrm{f}=0, \Delta \mathrm{f}=0.04, \Delta \mathrm{f}=0.10$ and $\Delta \mathrm{f}=0.37)$ and intensity contrasts $(\Delta \mathrm{i}=10$, $457 \Delta \mathrm{i}=20-30$ and $\Delta \mathrm{i}=40-50 \mathrm{~dB})$ used before. Neither of the conditions showed any 458 differences in the NIA of the responses to the deviant between the monotonic and the 459 non-monotonic neurons (Mann-Whitney rank sum test, $\mathrm{p}>0.1$ in all the cases but $460 \Delta \mathrm{f}=0.37$ at $\Delta \mathrm{i}=30$, where $\mathrm{p}=0.016$ ).

\section{The width of the frequency channel is frequency and intensity dependent}

463 2012), we also wished to check if this dependence affects the width of the frequency 464 channel. We analyzed if the frequency channels were wider at low- than at high 465 frequencies and if the frequency channels that code for higher intensities presented also 466 wider bandwidths than the ones than also code for lower intensities. To do so, we only 467 considered the neurons with significant SSA (CSI $\geq 0.18)$ and looked for any correlation 468 between the frequency and/or the intensity of the probe sound with the NIA values for 469 the deviant response when the conditioner was presented at a fixed intensity contrast $470(\Delta \mathrm{i}=30)$ at different frequency contrasts $(\Delta \mathrm{f}=0.04,0.1$ and 0.37 , Figure $7 \mathrm{~A})$. The results 471 demonstrate that the NIA values for the deviant response when the conditioner was at 472 a $\Delta \mathrm{i}=30$ with a $\Delta \mathrm{f}=0.04$ did not present a significant correlation with the frequency or 473 the intensity of the probe sound (Spearman rank order correlation, $p=0.38$ and $p=0.89$, 474 respectively). The same was observed when the conditioner was at a $\Delta \mathrm{i}=30$ with a $475 \Delta \mathrm{f}=0.37$ (Spearman rank order correlation, $\mathrm{p}=0.77$ and $\mathrm{p}=0.22$, respectively). As 476 expected, at a $\Delta \mathrm{f}=0.04$ the NIA values were close to 0 regardless of the frequency and 477 the intensity of the probe sound, while at a $\Delta \mathrm{f}=0.37$ the values were high regardless of 478 the frequency and the intensity of the probe. Interestingly, the trend disappeared when 479 we analyzed the data at $\Delta \mathrm{f}=0.1$ : the width of the frequency channels had a clear 480 dependence on the frequency and the intensity of the probe sound (Spearman rank order 481 correlation, $r_{\text {freq }}=0.239 r_{\text {int }}=-0.26 ; p \leq 0.05$ in both cases; Figure $7 C-D$, respectively). 482 Thus, while the frequency channel seems to generally cover the 0.057 octaves range 483 implicit in the 0.04 frequency contrast (regardless of the frequency and the intensity of 484 the probe sound), the 0.141 octaves range embedded in the $\Delta \mathrm{f}=0.1$ can lie either inside 
485 (at low frequencies and higher intensities) or outside the frequency channel (at high 486 frequencies and lower intensities, Figure 7A). On the other hand, the 0.526 octaves 487 range related with a $\Delta \mathrm{f}=0.37$ usually falls out the frequency channel, no matter what 488 the frequency or the intensity of the probe sound is.

\section{Neurons with high SSA levels have narrow frequency channels} rapid adaptation paradigm (RAP; see Methods and Figure 1C), that allows to compare the FRA and the area of frequencies and intensities capable of generating adaptation to the low intensity probe sound. Figure 8A shows an example of the FRA (left chart) and the area of suppression obtained with the RAP (upper right chart), where the probe sound is represented by a black dot over the charts. To confirm that the adaptation observed in the RAP is unrelated to forward suppression (Nelson et al., 2009), a twotone protocol was also tested in 7 of these neurons (in such protocol 2 sounds were presented and the probe sound was immediately presented after the conditioner, with a conditioner-probe delay of $0 \mathrm{~ms}$ ). The area of suppression of the two-tone protocol usually covered the whole FRA (Figure 8A, bottom right chart) and even a low intensity conditioner produced suppression of the probe sound. Thus, the areas of suppression were different between the RAP and the two-tone protocol, proving to be independent processes.

Thirty-three neurons were recorded with the RAP. Neurons with high levels of SSA (Figure 8B-C) showed a narrow frequency channel, while neurons with lower levels presented a broad frequency channel (Figure $8 \mathrm{D}-\mathrm{E}$ ). In order to quantify such

507 differences, we calculated ratio between the bandwidth of the frequency channel and 508 the FRA (Figure 8F-G). A simple regression of the bandwidth at 10 and $30 \mathrm{~dB}$ above 509 the probe sound show that the neurons with high frequency SSA sensitivity have 510 narrower frequency channels (Figure 8F). With the aim of quantify this trend, we 511 divided the neurons evaluated with the RAP in two groups, regarding its SSA 512 sensitivity. Thus, when we compared both populations we found that the frequency 513 channel in the neurons with high frequency SSA sensitivity $(n=21)$ barely covered a 514 quarter of the FRA at 10 and $30 \mathrm{~dB}$ reTh, while the frequency channels found in the 515 neurons with low frequency SSA sensitivity were broader (Figure 8G Mann-Whitney 516 rank sum test, $\mathrm{p}<0.05$ at both 10 and $30 \mathrm{~dB}$ reTh). Last, the bandwidth ratio 
517 demonstrated a narrow frequency channel in the high SSA neurons compared with the 518 neurons that showed low SSA.
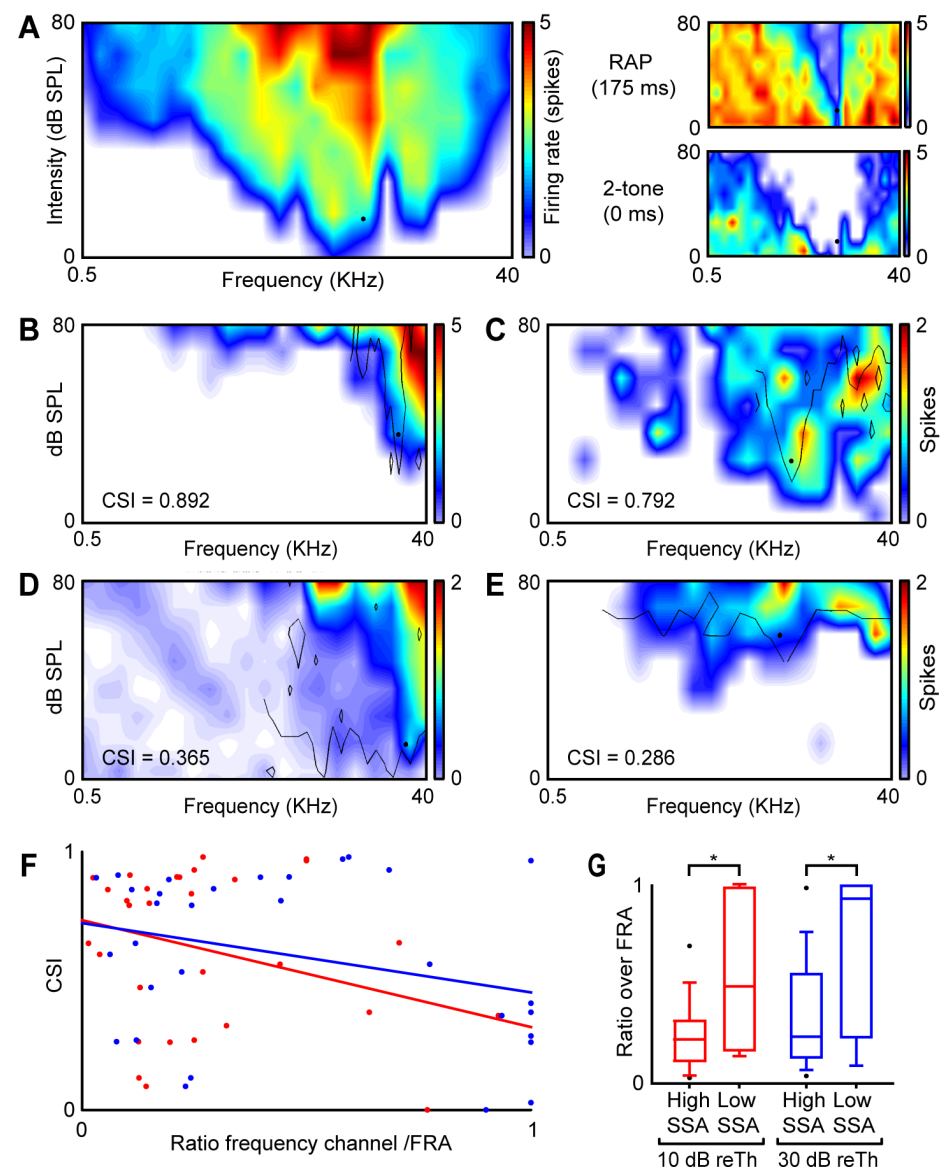

Figure 8. Rapid adaptation paradigm. A. A FRA of an IC neuron is shown in the left panel. The probe sound used in the RAP protocol is represented as a black dot over the FRA. In the right panels, responses to the probe sound are shown at a conditioner-probe delay of 1) $175 \mathrm{~ms}$ (RAP protocol; upper right, adaptive processes) and 2) $0 \mathrm{~ms}$ (2tones suppression; bottom right, forward suppression). The area of suppression obtained in the RAP protocol is defined as frequency channel. B-C. FRAs of two neurons with high frequency-deviant SSA with its corresponding frequency channels. D-E. FRAs of two neurons with low frequency deviant SSA and its corresponding frequency channels. Note that the width of the frequency channels is larger in D-E than in B-C. F. Correlation between the proportion of the FRA covered by the frequency channels against the CSI at 10- (red lines and dots) and $30 \mathrm{~dB}$ (blue lines and dots) over threshold. G. Proportion of the FRA covered by the frequency channels computed in the neurons with high- and low frequency deviant SSA. The bandwidth of the frequency channels at both 10 and $30 \mathrm{~dB}$ over threshold cover less frequency range of the FRA in the neurons with high frequency deviant SSA. 


\section{DISCUSSION}

520

521

522

523

524

525

526

527

528

529

530

531

532

533

534

535

536

537

538

539

540

541

542

543

544

545

546

547

548

549

Our results demonstrate that neither monotonic nor non-monotonic IC neurons show SSA for purely intensity deviant sounds, as they are not able to detect low intensity tones embedded within a sequence of the same tone at higher intensities. Nevertheless, the analysis of the double deviant data shed light on the across-adaptation caused from the high- to the low intensity sounds. Thus, SSA can be elicited if and when the high intensity conditioner sound is outside the frequency channels that code for the probe sound. The width of the channels is frequency- and intensity dependent, and neurons with high frequency SSA sensitivity present narrow frequency channels.

\section{Comparison with previous studies}

In the present account we demonstrate that neurons of the IC are sensitive to SSA for high intensity deviant sounds, as in the auditory cortex (Ulanovsky et al., 2003; Farley, 2010) but not to low intensity deviants. In the cortex however, and despite the pattern of neuronal responses reported in these two studies being similar, one study interprets as SSA for low intensity deviants (Ulanovsky et al., 2003) while another did not (Farley et al., 2010). The first claimed that the results were inconsistent with a purely adaptive phenomenon $\left(\mathrm{SI}_{\text {low }}+\mathrm{SI}_{\text {high }}>0\right)$ while the latter reported gain changes. Our results conform to the gain changes explanation (Sign test for $\mathrm{SI}_{\text {low }}+\mathrm{SI}_{\text {high }}=0$; $p=0.392$ ), demonstrating the absence of SSA for low intensity deviant sounds.

Näätänen's seminal paper (1978) demonstrated that MMN could be elicited by intensity increments and posterior works showed it also with intensity decrements (Näätänen et al., 1987, 1989a, 1989b; Paavilainen et al., 1991, 1993). An elegant paper (Jacobsen et al., 2003) demonstrated stimulus-specific MMN responses for both intensity increments and decrements, but they show that the P1-N1 component to the low intensity deviant was similar (or even smaller) to the same tone as standard. P1 and N1 components are attributed to basic auditory perception from the auditory cortex (Hari et al., 1984; Maess et al., 2007) and such reduced response conform to the data presented here. Middle latency responses (Althen et al., 2011) also showed MMN-like responses to intensity decrements between the $\mathrm{Na}$ and the $\mathrm{Pa}$ components, although the negative deflection observed by these authors (Figure 6C from Althen et al., 2011) could also be reflecting across-adaptation from high to low intensity sounds. 
If that is so, intensity coding would be dominated by across-adaptation from

551 high- to low intensities and genuine intensity discrimination (Jacobsen et al., 2003)

552 would be generated only at high order cortical areas. Considering that 1) true intensity

553 SSA neurons should respond better to both low- and high-intensity deviant sounds and

554 that 2) only 4 out of 117 neurons analyzed (3.4\%) showed a slightly larger sensitivity

555 to low intensity deviant sounds, we conclude that IC neurons do not present purely

556 intensity SSA.

\section{Frequency channel model in the inferior colliculus}

558 Since inhibition is only playing a key role in modulating SSA but not in its 559 generation (Pérez-González et al., 2012; Duque et al., 2014), a synaptic depression 560 fatigue model (Grill-Spector et al., 2006; Briley and Krumbholz, 2013) has been 561 proposed as the most likely explanation for SSA (Eytan et al., 2003; Mill et al., 2011a, 562 2011b), although more complex mechanisms may explain it at the cortical level (Taaseh 563 et al., 2011; Hershenhoren et al., 2014). However, the data shown in the present account 564 from the IC perfectly fits this model (Figure 9A). In the frequency domain, as long as 565 the repeated frequency is outside the frequency channel (Figure 9A, diamond) SSA 566 would be present (Figure 9B). In the intensity domain, regardless the intensity of the 567 repeated frequency (Figure 9A, square) across-adaptation from high- to low intensities 568 will always be present (Figure 9C). If we present a high intensity sound with a different 569 frequency (Figure 9A, triangle), SSA would depend on the width of the frequency 570 channel. If the repeated frequency is outside the frequency channel there will be no 571 across-adaptation; but if it is inside the resulting probe response will be reduced (Figure 572 9D). Interestingly, MMN responses to double deviants did not show additivity 573 (Paavilainen et al., 2001; Wolff and Schröger, 2001) which implies that MMN, as well 574 as SSA, do not process frequency and intensity information independently. Moreover, 575 the analysis of the N1 component provided a similar frequency channel model 576 (Näätänen et al., 1988; Herrmann et al., 2013, 2014), pointing out the similarities 577 between the adaptive processes in SSA and MMN. 

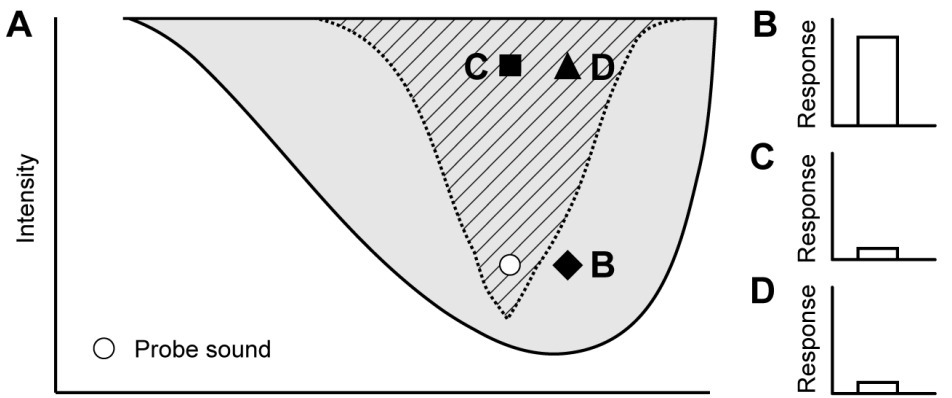

Frequency

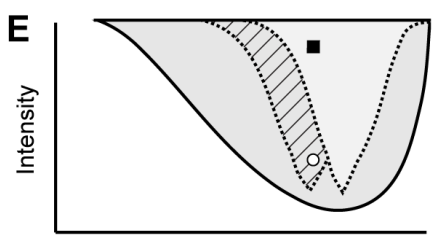

Frequency

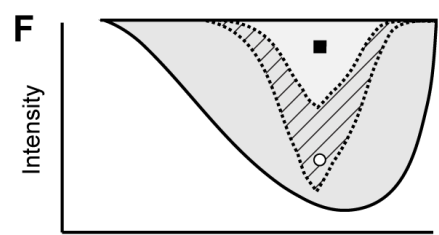

Frequency

Figure 9. Model of the frequency and intensity dependence of SSA in an IC neuron. A. Schematic FRA showing the response of an IC neuron. The probe sound is represented as a white circle and three different conditioner sounds are also drawn as black figures. The theoretical area of the frequency channel coding for the probe sound is represented as a region with diagonal lines. B. Response to the probe sound when the conditioner sound is in B. No cross adaptation is observed. C. Response to the probe sound when the conditioner sound is in C. Cross adaptation suppress the response to the probe sound. D. Response to the probe sound when the conditioner sound is in D. Cross adaptation could be observed depending on the width of the frequency channel. E-F. The reduced SSA observed at high intensities could be due to additional frequency channel that expanded at high intensities (E) or to specific high-intensity channels that do not usually show adaptation $(\mathbf{F})$. 6 and 8). As we have previously demonstrated that frequency SSA neurons present

580 broad FRAs (Duque et al., 2012), it is tempting to speculate that such neurons can integrate more frequency inputs. Moreover, the low levels of frequency SSA observed at high intensities (Duque et al., 2012) may be explained because the frequency channels broaden monotonically with intensity. We also showed that frequency channels are narrower at high frequencies, consequently increasing adaptation at high frequencies (Figure 7C-D), a phenomenon that has been previously observed in the auditory nerve fibers (Westerman and Smith, 1985) and the IC (Figure 5 from Dean et al., 2008; Figure 7C from Duque et al., 2012) and may be related with the great amount of high frequency behaviorally relevant sounds rat usually process. 
The current data support the idea that there is no SSA for intensity deviant sounds because of forward suppression-like phenomena. If that is so, adjustments to sound intensity statistics (Dean et al., 2005) could only be produced from low- to high intensity sounds. At first sight, this does not fit with the data presented by Dean and colleagues (2005) where, at a population level, bimodal stimuli adjust responses to incorporate both low- and high-intensity regions (Dean et al., 2005). Nevertheless, these authors commented that individual neurons did not show any obvious trend to adjust to both low- and high-intensity regions (Figure 4C from Dean et al., 2005).

SSA at the intensity domain greatly resembles forward suppression in the IC (Nelson et al., 2009), but some differences arise when comparing both studies. First, forward suppression would involve inhibitory mechanisms (Nelson et al., 2009), but we have previously demonstrated that SSA is not generated by GABAergic inhibition in both the IC (Pérez-González et al., 2012) and the thalamus (Duque et al., 2014). In

603 fact, as non-monotonic SSA neurons in the IC -generated by GABAergic inhibition 604 (Sivaramakrishnan, et al., 2004; Grimsley et al., 2013)- do not maintain responsiveness 605 to low intensity sounds embedded in a background of loud sounds, inhibitory 606 generation of non-monotonicity in the IC would be a post hoc phenomenon independent 607 of the excitatory inputs that generate SSA. Nevertheless, such non-monotonicity could 608 eventually lead to deviant detection at more high-level relay stations of the auditory 609 system, like the auditory cortex (Watkins and Barbour, 2008; 2011a; 2011b). Secondly, 610 forward suppression in the IC is evident up to $\sim 70 \mathrm{~ms}$ conditioner-probe delays (Nelson 611 et al., 2009). In the present account, delays of $175 \mathrm{~ms}$ were used between the sounds, a 612 condition that in the IC only showed a $\sim 5 \mathrm{~dB}$ residual masking (Nelson et al., 2009).

613 Finally, forward suppression experiment were conducted in central nucleus IC-like 614 neurons (Nelson et al., 2009), while our SSA data population is biased to non-lemniscal 615 regions of the IC (Malmierca et al., 2009; Duque et al., 2012; Pérez-González et al., 2012; Ayala et al., 2013). In contrast, experiments performed in the auditory cortex (Calford and 618 Semple, 1995; Brosch and Schreiner, 1997; Scholl et al., 2008; Scholes et al., 2011) 619 suggest that forward suppression effects with conditioner-probe intervals higher than $620 \quad 100-150 \mathrm{~ms}$ are attributable to SSA, probably through synaptic depression (Wehr and 
621 Zador, 2005; Scholes et al., 2011). If forward suppression is a merely adaptive process,

622 the absence of intensity SSA would be determined by the overlap in the synapses

623 activated by high- and low intensity sounds (Scholl et al., 2008). Indeed, the dynamics

624 of adaptation for forward suppression, intensity SSA and dynamic range adjustments

625 are virtually identical. The three phenomena seem to all share dual adaptations that

626 comprise 1) an input related mechanism (i.e., synaptic depression) and 2) a gain control

627 mechanism (i.e., inhibition), where the input related component is generally more

628 relevant (SSA: Ulanovsky et al., 2003; Pérez-González et al., 2012; forward

629 suppression: Scholl et al., 2008; dynamic range adjustment: Wen et al., 2009). Such

630 dual adaptation is also reflected in the similar time constants obtained when evaluating

631 the time course of adaptation (Ulanovsky et al., 2004; Dean et al., 2008).

632 In summary, our data indicates that a dynamic range adjustment to intensity

633 (Dean et al., 2005) is passively due to SSA (Condon and Weinberger, 1991; Malone

634 and Semple, 2001; Ulanovsky et al., 2003; Malmierca et al., 2009), a phenomenon

635 present for frequency- but not for intensity-deviant tones and that may provide a likely

636 explanation for central forward suppression in the IC. 


\section{REFERENCES}

Althen H, Grimm S, Escera C (2011) Fast detection of unexpected sound intensity decrements as revealed by human evoked potentials. PLoS One 6:e28522.

Antunes FM, Malmierca MS (2014) An Overview of Stimulus-Specific Adaptation in the Auditory Thalamus. Brain topography.

Antunes FM, Nelken I, Covey E, Malmierca MS (2010) Stimulus-specific adaptation in the auditory thalamus of the anesthetized rat. PLoS One 5:e14071.

Ayala YA, Malmierca MS (2013) Stimulus-specific adaptation and deviance detection in the inferior colliculus. Front Neural Circuits 6:89.

Ayala YA, Perez-Gonzalez D, Duque D, Nelken I, Malmierca MS (2013) Frequency discrimination and stimulus deviance in the inferior colliculus and cochlear nucleus. Front Neural Circuits 6:119.

Briley PM, Krumbholz K (2013) The specificity of stimulus-specific adaptation in human auditory cortex increases with repeated exposure to the adapting stimulus. J Neurophysiol 110:2679-2688.

Brosch M, Schreiner CE (1997) Time course of forward masking tuning curves in cat primary auditory cortex. J Neurophysiol 77:923-943.

Calford MB, Semple MN (1995) Monaural inhibition in cat auditory cortex. J Neurophysiol 73:1876-1891.

Condon CD, Weinberger NM (1991) Habituation produces frequency-specific plasticity of receptive fields in the auditory cortex. Behavioral neuroscience 105:416-430.

Dahmen JC, Keating P, Nodal FR, Schulz AL, King AJ (2010) Adaptation to stimulus statistics in the perception and neural representation of auditory space. Neuron 66:937-948.

de la Rocha J, Marchetti C, Schiff M, Reyes AD (2008) Linking the response properties of cells in auditory cortex with network architecture: cotuning versus lateral inhibition. J Neurosci 28:9151-9163.

Dean I, Harper NS, McAlpine D (2005) Neural population coding of sound level adapts to stimulus statistics. Nat Neurosci 8:1684-1689.

Dean I, Robinson BL, Harper NS, McAlpine D (2008) Rapid neural adaptation to sound level statistics. J Neurosci 28:6430-6438. 
Duque D, Malmierca MS, Caspary DM (2014) Modulation of stimulus-specific adaptation by GABAA receptor activation or blockade in the medial geniculate body of the anaesthetized rat. J Physiol 592:729-743.

Duque D, Perez-Gonzalez D, Ayala YA, Palmer AR, Malmierca MS (2012) Topographic distribution, frequency, and intensity dependence of stimulusspecific adaptation in the inferior colliculus of the rat. J Neurosci 32:1776217774.

Escera C, Malmierca MS (2013) The auditory novelty system: An attempt to integrate human and animal research. Psychophysiology 51:111-123.

Farley BJ, Quirk MC, Doherty JJ, Christian EP (2010) Stimulus-specific adaptation in auditory cortex is an NMDA-independent process distinct from the sensory novelty encoded by the mismatch negativity. J Neurosci 30:16475-16484.

Faure PA, Fremouw T, Casseday JH, Covey E (2003) Temporal masking reveals properties of sound-evoked inhibition in duration-tuned neurons of the inferior colliculus. J Neurosci 23:3052-3065.

Grill-Spector K, Henson R, Martin A (2006) Repetition and the brain: neural models of stimulus-specific effects. Trends Cogn Sci 10:14-23.

Grimsley CA, Sanchez JT, Sivaramakrishnan S (2013) Midbrain local circuits shape sound intensity codes. Front Neural Circuits 7:174.

Hara K, Harris RA (2002) The anesthetic mechanism of urethane: the effects on neurotransmitter-gated ion channels. Anesth Analg 94:313-318, table of contents.

Hari R, Hamalainen M, Ilmoniemi R, Kaukoranta E, Reinikainen K, Salminen J, Alho K, Naatanen R, Sams M (1984) Responses of the primary auditory cortex to pitch changes in a sequence of tone pips: neuromagnetic recordings in man. Neurosci Lett 50:127-132.

Hernandez O, Espinosa N, Perez-Gonzalez D, Malmierca MS (2005) The inferior colliculus of the rat: a quantitative analysis of monaural frequency response areas. Neuroscience 132:203-217.

Herrmann B, Henry MJ, Obleser J (2013) Frequency-specific adaptation in human auditory cortex depends on the spectral variance in the acoustic stimulation. $\mathrm{J}$ Neurophysiol 109:2086-2096.

Herrmann B, Schlichting N, Obleser J (2014) Dynamic range adaptation to spectral stimulus statistics in human auditory cortex. J Neurosci 34:327-331. 
Hershenhoren I, Taaseh N, Antunes FM, Nelken I (2014) Intracellular correlates of stimulus-specific adaptation. J Neurosci 34:3303-3319.

Jacobsen T, Horenkamp T, Schroger E (2003) Preattentive memory-based comparison of sound intensity. Audiol Neurootol 8:338-346.

Loewy DH, Campbell KB, de Lugt DR, Elton M, Kok A (2000) The mismatch negativity during natural sleep: intensity deviants. Clin Neurophysiol 111:863-872.

Loftus WC, Malmierca MS, Bishop DC, Oliver DL (2008) The cytoarchitecture of the inferior colliculus revisited: a common organization of the lateral cortex in rat and cat. Neuroscience 154:196-205.

Maess B, Jacobsen T, Schroger E, Friederici AD (2007) Localizing pre-attentive auditory memory-based comparison: magnetic mismatch negativity to pitch change. NeuroImage 37:561-571.

Malmierca M, Ryugo D (2011) Auditory System. In: The Mouse Nervous System 1st Edition, pp 607-645. San Diego, CA: Academic Press.

Malmierca MS, Seip KL, Osen KK (1995) Morphological classification and identification of neurons in the inferior colliculus: a multivariate analysis. Anat Embryol (Berl) 191:343-350.

Malmierca MS, Cristaudo S, Perez-Gonzalez D, Covey E (2009) Stimulus-specific adaptation in the inferior colliculus of the anesthetized rat. J Neurosci 29:5483-5493.

Malmierca MS, Blackstad TW, Osen KK, Karagulle T, Molowny RL (1993) The central nucleus of the inferior colliculus in rat: a Golgi and computer reconstruction study of neuronal and laminar structure. J Comp Neurol 333:127.

Malmierca MS, Hernandez O, Falconi A, Lopez-Poveda EA, Merchan M, Rees A (2003) The commissure of the inferior colliculus shapes frequency response areas in rat: an in vivo study using reversible blockade with microinjection of kynurenic acid. Exp Brain Res 153:522-529.

Malone BJ, Semple MN (2001) Effects of auditory stimulus context on the representation of frequency in the gerbil inferior colliculus. J Neurophysiol 86:1113-1130.

Mill R, Coath M, Wennekers T, Denham SL (2011a) Abstract Stimulus-Specific Adaptation Models. Neural Comput. 
Mill R, Coath M, Wennekers T, Denham SL (2011b) A neurocomputational model of stimulus-specific adaptation to oddball and Markov sequences. PLoS Comput Biol 7:e1002117.

Näätänen R, Paavilainen P, Reinikainen K (1989a) Do event-related potentials to infrequent decrements in duration of auditory stimuli demonstrate a memory trace in man? Neurosci Lett 107:347-352.

Näätänen R, Paavilainen P, Alho K, Reinikainen K, Sams M (1987) The mismatch negativity to intensity changes in an auditory stimulus sequence. Electroencephalography and clinical neurophysiology Supplement 40:125131.

Näätänen R, Paavilainen P, Alho K, Reinikainen K, Sams M (1989b) Do event-related potentials reveal the mechanism of the auditory sensory memory in the human brain? Neurosci Lett 98:217-221.

Näätänen R, Sams M, Alho K, Paavilainen P, Reinikainen K, Sokolov EN (1988) Frequency and location specificity of the human vertex N1 wave. Electroencephalogr Clin Neurophysiol 69:523-531.

Näätänen R (1992) Attention and Brain Function. Hillsdale, NJ: Erlbaum.

Näätänen R, Gaillard AW, Mantysalo S (1978) Early selective-attention effect on evoked potential reinterpreted. Acta Psychol (Amst) 42:313-329.

Nelken I (2014) Stimulus-specific adaptation and deviance detection in the auditory system: experiments and models. Biol Cybern.

Nelson PC, Smith ZM, Young ED (2009) Wide-dynamic-range forward suppression in marmoset inferior colliculus neurons is generated centrally and accounts for perceptual masking. J Neurosci 29:2553-2562.

Paavilainen P, Valppu S, Naatanen R (2001) The additivity of the auditory feature analysis in the human brain as indexed by the mismatch negativity: $1+1$ approximately 2 but $1+1+1<3$. Neurosci Lett 301:179-182.

Paavilainen P, Jiang D, Lavikainen J, Naatanen R (1993) Stimulus duration and the sensory memory trace: an event-related potential study. Biol Psychol 35:139152.

Paavilainen P, Alho K, Reinikainen K, Sams M, Naatanen R (1991) Right hemisphere dominance of different mismatch negativities. Electroencephalogr Clin Neurophysiol 78:466-479. 
Paxinos G, Watson C (2005) The rat brain in stereotaxic coordinates. Burlington: Elsevier-Academic.

Perez-Gonzalez D, Hernandez O, Covey E, Malmierca MS (2012) GABA(A)-Mediated Inhibition Modulates Stimulus-Specific Adaptation in the Inferior Colliculus. PLoS One 7:e34297.

Pérez-González D, Malmierca MS, Covey E (2005) Novelty detector neurons in the mammalian auditory midbrain. Eur J Neurosci 22:2879-2885.

Rabinowitz NC, Willmore BD, Schnupp JW, King AJ (2011) Contrast gain control in auditory cortex. Neuron 70:1178-1191.

Reches A, Gutfreund Y (2008) Stimulus-specific adaptations in the gaze control system of the barn owl. J Neurosci 28:1523-1533.

Scholes C, Palmer AR, Sumner CJ (2011) Forward suppression in the auditory cortex is frequency-specific. Eur J Neurosci.

Schroger E, Wolff C (1996) Mismatch response of the human brain to changes in sound location. Neuroreport 7:3005-3008.

Simpson AJ, Harper NS, Reiss JD, McAlpine D (2014) Selective adaptation to "oddball" sounds by the human auditory system. J Neurosci 34:1963-1969.

Sivaramakrishnan S, Sterbing-D'Angelo SJ, Filipovic B, D'Angelo WR, Oliver DL, Kuwada S (2004) GABA( A) synapses shape neuronal responses to sound intensity in the inferior colliculus. J Neurosci 24:5031-5043.

Taaseh N, Yaron A, Nelken I (2011) Stimulus-specific adaptation and deviance detection in the rat auditory cortex. PLoS One 6:e23369.

Ulanovsky N, Las L, Nelken I (2003) Processing of low-probability sounds by cortical neurons. Nat Neurosci 6:391-398.

Ulanovsky N, Las L, Farkas D, Nelken I (2004) Multiple time scales of adaptation in auditory cortex neurons. J Neurosci 24:10440-10453.

Wark B, Lundstrom BN, Fairhall A (2007) Sensory adaptation. Curr Opin Neurobiol 17:423-429.

Watkins PV, Barbour DL (2008) Specialized neuronal adaptation for preserving input sensitivity. Nat Neurosci 11:1259-1261.

Watkins PV, Barbour DL (2011a) Level-tuned neurons in primary auditory cortex adapt differently to loud versus soft sounds. Cereb Cortex 21:178-190.

Watkins PV, Barbour DL (2011b) Rate-level responses in awake marmoset auditory cortex. Hear Res 275:30-42. 
Wehr M, Zador AM (2005) Synaptic mechanisms of forward suppression in rat auditory cortex. Neuron 47:437-445.

Wen B, Wang GI, Dean I, Delgutte B (2009) Dynamic range adaptation to sound level statistics in the auditory nerve. J Neurosci 29:13797-13808.

Wen B, Wang GI, Dean I, Delgutte B (2012) Time course of dynamic range adaptation in the auditory nerve. J Neurophysiol 108:69-82.

Westerman LA, Smith RL (1985) Rapid adaptation depends on the characteristic frequency of auditory nerve fibers. Hear Res 17:197-198.

Wolff C, Schroger E (2001) Human pre-attentive auditory change-detection with single, double, and triple deviations as revealed by mismatch negativity additivity. Neurosci Lett 311:37-40.

$\mathrm{Xu} \mathrm{X}, \mathrm{Yu} \mathrm{X}, \mathrm{He}$ J, Nelken I (2014) Across-ear stimulus-specific adaptation in the auditory cortex. Front Neural Circuits 8:89.

Yaron A, Hershenhoren I, Nelken I (2012) Sensitivity to complex statistical regularities in rat auditory cortex. Neuron 76:603-615.

Zhou Y, Wang X (2014) Spatially extended forward suppression in primate auditory cortex. Eur J Neurosci 39:919-933. 



\section{DISCUSSION}



The present $\mathrm{PhD}$ thesis summarizes the results of 4 different electrophysiological studies performed in rats or mice at two different subcortical nuclei: the IC and the MGB.

In the first study (Duque et al., 2012) I demonstrate that, in the IC, SSA is not constant within the FRA, and therefore it is not an intrinsic property of the neuron. I further demonstrate that higher levels of SSA are biased towards low intensity levels and to the high-frequency edge of the FRA, and that ON responses exhibit larger SSA values than other response types. I also demonstrate that neurons with broader FRAs show more SSA, and most of them are located in the cortical regions of the IC.

The second study (Duque et al., 2014) shows that the activation of $\mathrm{GABA}_{A}$ receptors with $\mathrm{GABA}$ or gaboxadol (GABA receptor agonist) increases the level of SSA in the MGB by decreasing the neuronal firing rate. Conversely, gabazine application $\left(G A B A_{A}\right.$ receptor antagonist) decreases the SSA and increases the firing rate. I conclude that the GABAergic system is exerting a gain control effect over the SSA sensitivity.

Study II (Duque and Malmierca, 2014) reveals SSA levels in the IC of the awake mouse equivalent to those seen in the anesthetized mouse and rat. I also show that urethane anesthesia does affect the spontaneous rate (SR), which is dynamically modulating SSA by the animal's state.

Last, study IV (Wang et al., 2014, under review) shows that IC neurons do not present SSA for purely intensity deviant sounds, as they are not able to detect low intensity tones embedded within a sequence of the same tone at higher intensities. Nevertheless, SSA can be elicited if and when the high intensity conditioner sound is outside the frequency channels that code for the low intensity sound. I also show that the width of the channels is frequency- and intensity dependent, and that neurons with high frequency SSA sensitivity present narrow frequency channels. 


\section{Neuronal properties of SSA}

In the first study (Duque et al., 2012) I confirm that neurons from the non-lemniscal pathway in the IC have higher frequency SSA sensitivity (Malmierca et al., 2009). Interestingly, the last study of this PhD thesis (Wang et al., 2014, under review) shows that neurons with high frequency SSA sensitivity present narrow frequency channels. As neurons in the LCIC, DCIC and $\mathrm{RCIC}$ have the largest and less oriented dendritic arbors in the IC (Malmierca et al., 1993; 1995; 2011), it is tempting to speculate that such 'disorientation' allows the neurons to integrate more (Duque et al., 2012) and thinner (Wang et al., 2014, under review) frequency inputs and thus, that the higher levels of SSA found in the cortical regions of the IC are accordingly related to the width of the FRA.

The first study also shows that low sound levels present the higher SSA sensitivity (Duque et al., 2012). At low intensities, the response to the standard stimulus disappears gradually because of the adaptation process, resulting in high CSI. At high intensities the response to the standard stimulus is more sustained over time and the net result is a low CSI value. The study also confirms that SSA is not only stronger for the ON responses of the neurons (Duque et al., 2012) but that the ON portion of other response types with a well-defined ON region, like ON-OFF and ON-SUSTAINED responses show larger SSA as well. Nevertheless, such firing rate dependence of SSA is independent of the intensity of the sound (Duque et al., 2012). In the last study (Wang et al., 2014, under review) I further demonstrate that frequency channels broaden monotonically with intensity. Thus, a likely explanation for the differences in frequency SSA sensitivity between low and high intensities is that frequency channels allow across-frequency adaptation at low intensity levels, but not at high intensities, where the channel will be wider than the frequency contrasts used in the first study (Duque et al., 2012). 
I also demonstrate that IC neurons are sensitive to SSA for high intensity deviant sounds, as in the auditory cortex (Ulanovsky et al., 2003; Farley, 2010) but not to low intensity deviants (Wang et al., 2014, under review). The data suggests that there is no SSA for intensity deviant sounds because of forward suppression-like phenomena, where the absence of intensity SSA would be determined by the overlap in the synapses activated by high- and low intensity sounds (Scholl et al., 2008). Thus, intensity coding seems to be dominated by across-adaptation from high- to low intensities and genuine intensity discrimination (Jacobsen et al., 2003) would only be generated at high order cortical areas.

To further understand the physical characteristics of the sound that defines SSA, I also tried to evoke SSA responses with double deviants for both frequency and intensity (Wang et al., 2014, under review). The analysis of the double-deviant data shed light on the across-adaptation caused from the high- to the low intensity sounds. Thus, I found that some doubledeviants at particular combinations of frequency and intensity can evoke SSA (Wang et al., 2014, under review). Interestingly, the synaptic depression model (Grill-Spector et al., 2006; Briley and Krumbholz, 2013) and the frequency-specific adaptation channels theory (Eytan et al., 2003; Mill et al., 2011a, 2011b; Taaseh et al., 2011; Hershenhoren et al., 2014) perfectly explain all the data commented above from studies I and IV. In the frequency domain, as long as the repeated frequency is outside the frequency channel (Figure 13A, diamond) there will be no across-adaptation, the probe sound would show a high response rate and SSA would be present (Figure 13B). In the intensity domain, regardless of the intensity of the repeated frequency (Figure 13A, square), across-adaptation from high to low intensities will always be present (Figure 13C). On the other hand, if we present a high intensity tone with a different frequency (Figure 13A, triangle), SSA would depend on the width of the frequency channel. If the repeated frequency is 
outside the frequency channel, there will be no across-adaptation; if it is inside, the resulting probe response will be reduced (Figure 13D).

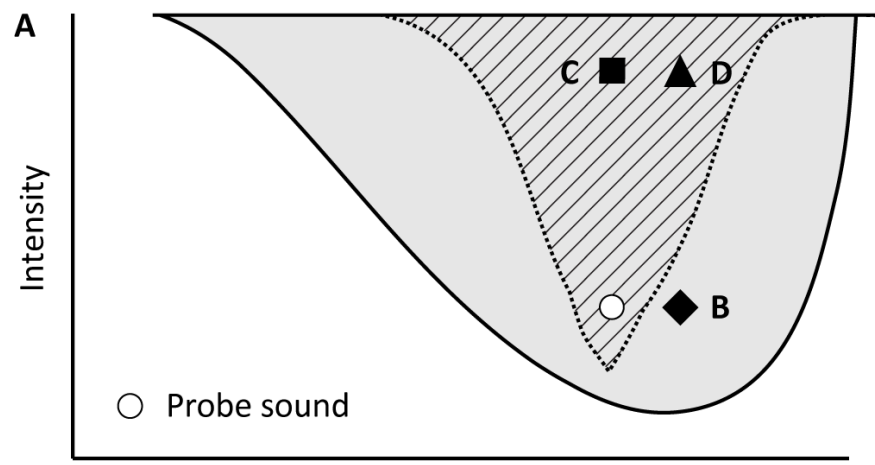

B

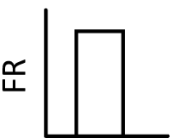

C

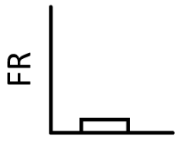

Frequency

D

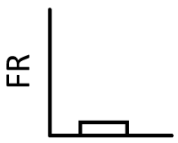

Figure 13. Frequency channel model of SSA. A. Schematic FRA showing the response of an IC neuron. The probe sound is represented as a white circle and three different conditioner sounds are drawn as black figures. The frequency channel for the probe tone is represented as a region with diagonal lines. B. Normal response to the probe sound when the conditioner sound is in B.C. Response to the probe sound when the conditioner tone is in C. Cross adaptation suppress the response. D. Response to the probe sound when the conditioner sound is in D. Cross adaptation could be observed depending on the width of the frequency channel.

I further demonstrated that frequency SSA sensitivity is stronger on the high-frequency edge of the FRA (Duque et al., 2012), possibly because the frequency channels are narrower at high frequencies (Wang et al., 2014, under review). Such phenomenon has been also observed in the auditory nerve fibers (Westerman and Smith, 1985) and the IC (Dean et al., 2008). High frequencies are behaviorally relevant sounds for the rats and may require a better response, hence increasing the sensitivity to high frequency sounds and developing hyperacuity and higher levels of frequency SSA.

Although it seemed clear that synaptic depression mechanisms are generating SSA at subcortical levels, we wanted to understand the actual role of inhibition in the modulation or generation of SSA. Thus, in order to check that possibility I used the microiontophoretic technique to record neurons while activating or blocking $\mathrm{GABA}_{A}$ receptors (Duque et al., 2014). 


\section{GABAergic modulation of SSA}

In the second study, I found that both GABA and gaboxadol increase the level of SSA in the MGB. On the other hand, the application of gabazine increased the neuronal firing rate and decreased the levels of SSA. As I show similar effects on SSA after GABA and gaboxadol (GABA $A_{A}$ selective agonist) application, it is unlikely that these actions are mediated through $G_{A B}$ receptors. In addition, as gabazine $\left(G_{A B A}\right.$ antagonist) does not block $G A B A_{B}$ receptors, it reinforces the idea that $\mathrm{GABA}_{B}$ receptors are not prominently involved in SSA coding.

In study II the change in SSA sensitivity by the manipulation of the $\mathrm{GABA}_{\mathrm{A}}$ receptor was not frequency specific: similar effects on the SSA sensitivity were apparent over the whole range of frequencies of the neuron (Duque et al., 2014), suggesting that GABAergic inhibition is a phenomenon independent of the excitatory inputs that generate SSA. In concord with this data, I also show that non-monotonic SSA neurons in the IC -generated by GABAergic inhibition (Sivaramakrishnan, et al., 2004; Grimsley et al., 2013)do not maintain responsiveness to low intensity sounds when embedded in a high intensity background (Wang et al., 2014, under review). Altogether, it is tempting to speculate that inhibition in the IC is happening a posteriori of the excitatory inputs that generate SSA.

In conclusion, the GABA effects shown in the MGB study (Duque et al., 2012) are similar to the ones observed in the IC (Pérez-González et al., 2012). The data from the second study -in conform with the IC data- show that the GABAergic system does not generate or create SSA de novo, but exerts a gain control function (Robinson and McAlpine, 2009; Isaacson and Scanziani, 2011). Such gain control decreases the responses to both common and novel stimuli (Figure 14), increasing the ratio between the responses, as explained by the 'iceberg effect' (Rose and Blakemore, 1974). 


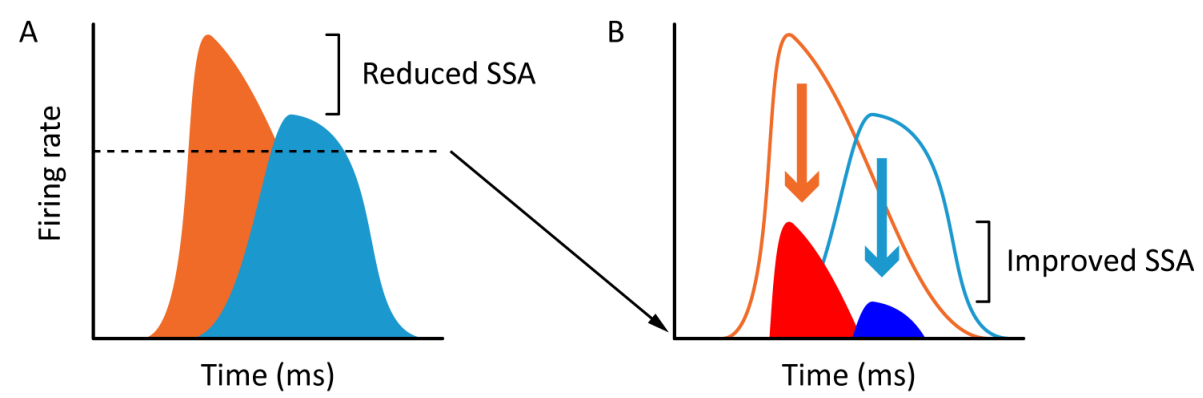

Figure 14. SSA modulation by inhibition. (A) In the absence of inhibition, responses to deviant (orange) and standard sounds (light blue). (B) Inhibition reduces the responses to both deviants (red) and standards (dark blue) increasing the deviant to standard ratio and thus enhancing SSA. From Pérez-González et al., 2012.

\section{The effects of anesthesia on SSA}

As anesthesia may be also altering the balance between excitation and inhibition, I implemented a system to record in awake animals to check if SSA was affected by anesthesia (Study III; Duque and Malmierca, 2014). This study shows that the high levels of SSA found in the mouse IC are similar to the ones seen in the urethane-anesthetized mouse and rat. It further reveals that urethane anesthesia does affect other response properties, as the SR. Our data is in accord with previous work that suggests a basal adaptive state due to high SR (Abolafia et al., 2011). This pre-adaptive situation will produce less adaptation to both the standard and deviant stimuli, decreasing the deviant-standard ratio, therefore reducing the levels of SSA.

Since high SR in the awake behaving animals are related to a decline in adaptation (Chung et al., 2002; Castro-Alamancos, 2004), attention during task engagement may modulate the neuronal responses (Buran et al., 2014). In this respect, I found a clear correlation between the CSI levels and the SR such that the higher the CSI level, the lower the SR (Duque and Malmierca, 2014). Thus, I speculate that attention might be dynamically increasing the SR, consequently reducing the levels of SSA. One plausible mechanism would be through GABAergic inhibition. As I demonstrate in the second study that 
$\mathrm{GABA}_{\mathrm{A}}$ mediated inhibition acts as a gain control system that enhances SSA (Pérez-Gonzalez et al., 2012; Duque et al., 2014), it could increase SSA by suppressing the SR. Moreover, recent studies have shown that the moderate urethane effects on neurotransmission seem to perfectly mimic natural sleep (Clement et al., 2008; Pagliardini et al., 2012; 2013), a state that implies a reduction of behavioral responsiveness (Rechtschaffen et al., 1966). Thus, I hypothesized that during sleep we may need more robust resources to react to danger (Duque and Malmierca, 2014) and attenuation of SR during sleep could improve this essential response.

\section{Synaptic mechanisms underlying SSA and future experiments}

In future research it would be interesting to understand the synaptic mechanisms underlying SSA. Synaptic depression could explain SSA (Chung et al., 2002; Rothman et al., 2009) as it is an input-specific mechanism and neural responses depend on the previous history of afferent firing (Abbott et al., 1997; Rothman et al., 2009). Synaptic depression scales neuronal sensitivity to all of its driving inputs (Abbott et al., 1997; Rothman et al., 2009), explaining a variety of time scales of adaptation (Varela et al., 1997).

Nevertheless, synaptic depression does not necessary have to be a passive phenomenon, as several different retrograde signaling pathways can act at the synaptic level. Gases (nitric oxide), peptides (dynorphin), growth factors (brain-derived neurotrophic factor), or conventional amino acid transmitters (glutamate or GABA) are released by postsynaptic neurons and then act on the axon terminals of presynaptic neurons (Regehr et al. 2009). In the mammalian brain, endocannabinoid (eCB) signaling enables neurons to regulate the strength of their inputs in a retrograde manner (Wilson and Nicoll, 2002; Freund et al., 2003; Kano et al., 2009).

The presynaptic localization of CB1 receptors and its inhibitory effect on neurotransmitter release have proved to be a general feature of most 
axon terminals in the central system (Kano et al. 2009), suppressing synaptic strength for tens of seconds (Wilson and Nicoll, 2001). Such ecB are released from the somatodendritic domain of neurons and then modify release properties of afferent axon terminals or regulate activity in nearby glial processes (Figure 15). Sustained firing of sodium spikes or burst firing can evoke eCB release and may contribute to short-term synaptic plasticity (Castillo et al., 2012).
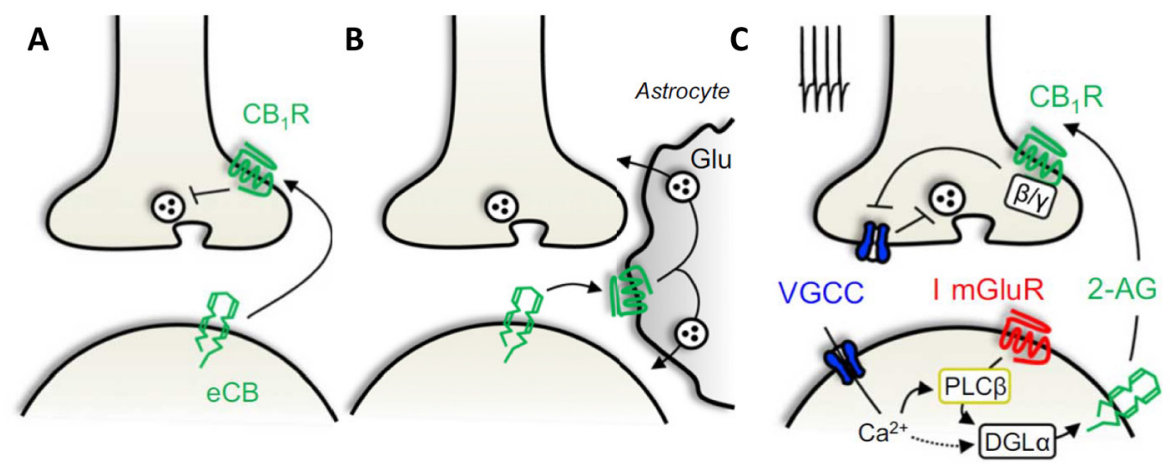

Figure 15. Endocannabinoid Signaling. A. Retrograde endocannabinoid (eCB) signaling. eCBs are mobilized from postsynaptic neurons and target presynaptic cannabinoid type 1 receptors (CB1Rs) to suppress neurotransmitter release. B. Neuron-astrocyte signaling. eCBs released from postsynaptic neurons stimulates astrocytic CB1Rs, thereby triggering gliotransmission. Glu, glutamate. C. Short-term depression. Postsynaptic activity triggers $\mathrm{Ca}^{2+}$ influx via voltage-gated $\mathrm{Ca}^{2+}$ channels. Other $\mathrm{Ca}^{2+}$ sources, like NMDARs and internal stores, may contribute. $\mathrm{Ca}^{2+}$ promotes eCB production. Presynaptic activity can also lead to eCB mobilization by activating postsynaptic glutamate receptors (mGluRs). Arachidonoylglycerol (2-AG) is released and retrogradely targets presynaptic CB1Rs. Adapted from Castillo et al., 2012.

Future research is needed to understand if retrograde transmission, as the one mediated by the ECB system, is generating SSA at subcortical levels. Interestingly, eCB signaling have been proved to act in the auditory pathway at the level of the cochlear nucleus (Zhao et al., 2009; Zhao and Tzounopoulos, 2011), the superior olivary complex (Trattner et al., 2013) and the external cortex of the IC of the barn owl (Penzo and Peña, 2009). 


\section{Final remarks}

In conclusion, this Doctoral Thesis demonstrates that SSA is a genuine phenomenon from the mammalian brain (rat, mouse...) that it is not artifact of anesthesia and that it is modulated by the GABAergic system. This work further demonstrates that SSA is likely explained at the level of the IC and the MGB by the frequency channel model. It also suggests that SSA could be generated in a bottom-up fashion throughout the auditory pathway, as it seems to be an input related phenomenon. The existence of consecutive gain controls over SSA in the IC and the MGB suggests the existence of successive hierarchical levels of processing through the auditory system that would allow reduction of redundant information. Therefore, SSA would act as a preattentive gating involved in reducing sensory input to behaviorally relevant sound for the animals, helping to provide adequate responses for survival. 



\section{CONCLUSIONS}



1. SSA is not constant within the FRA, and therefore is not a characteristic property of the neuron. SSA is stronger at low intensity sounds and biased towards high frequencies.

2. Neurons located in the non-lemniscal regions of the IC show the highest levels of SSA, an area characterized by discrete onset neuronal temporal responses and broad FRAs.

3. The GABAergic system modulates SSA at the level of the MGB in a gain control manner. Thus, GABA decreases the neuronal firing rate and increase the level of SSA, but do not generate it.

4. GABA produces its modulation mainly through $\mathrm{GABA}_{\mathrm{A}}$ receptors.

5. SSA is a genuine phenomenon and not an artifact attributable to anesthesia. SSA levels in the awake mice and the anesthetized mice and rat are comparable.

6. SSA is dynamically modulated by the animal's state through the neuronal SR.

7. IC neurons do not show SSA for purely intensity deviant sounds, but SSA can be elicited if and when the standard sound is outside the frequency channels that code for the deviant sound.

8. Neurons with high frequency SSA sensitivity present narrow frequency channels. Frequency channels broaden monotonically with intensity and are narrower at high frequencies. 



\section{REFERENCES}



Abolafia, J.M., Vergara, R., Arnold, M.M., Reig, R. and Sanchez-Vives, M.V. (2011) Cortical auditory adaptation in the awake rat and the role of potassium currents. Cereb Cortex, 21, 977-990.

Abbott, L.F., Varela, J.A., Sen, K. and Nelson, S.B. (1997) Synaptic depression and cortical gain control. Science 275: 220-224.

Aitkin, L.M. (1973) Medial geniculate body of the cat: responses to tonal stimuli of neurons in medial division. J. Neurophysiol. 36: 275-283.

Aitkin, L.M., Dickhaus, H., Schult, W. and Zimmermann, M. (1978) External nucleus of inferior colliculus: auditory and spinal somatosensory afferents and their interactions. J Neurophysiol, 41, 837-847.

Aitkin, L.M., Kenyon, C.E. and Philpott, P. (1981) The representation of the auditory and somatosensory systems in the external nucleus of the cat inferior colliculus. J Comp Neurol, 196, 25-40.

Alitto, H.J. and Usrey, W.M. (2003) Corticothalamic feedback and sensory processing. Curr. Opin. Neurobiol. 13: 440-445.

Anderson, L.A., Christianson, G.B. and Linden, J.F. (2009) Stimulus-specific adaptation occurs in the auditory thalamus. J Neurosci, 29, 7359-7363.

Anderson, L.A. and Malmierca, M.S. (2013) The effect of auditory cortex deactivation on stimulus-specific adaptation in the inferior colliculus of the rat. Eur J Neurosci, 37, 52-62.

Anderson, L.A., Malmierca, M.S., Wallace, M.N. and Palmer, A.R. (2006) Evidence for a direct, short latency projection from the dorsal cochlear nucleus to the auditory thalamus in the guinea pig. Eur J Neurosci, 24, 491-498. 
Antunes, F.M. and Malmierca, M.S. (2011) Effect of auditory cortex deactivation on stimulus-specific adaptation in the medial geniculate body. J Neurosci, 31, 17306-17316.

Antunes, F.M. and Malmierca, M.S. (2014) An Overview of Stimulus-Specific Adaptation in the Auditory Thalamus. Brain Topogr. 27(4):480-99.

Antunes, F.M., Nelken, I., Covey, E. and Malmierca, M.S. (2010) Stimulusspecific adaptation in the auditory thalamus of the anesthetized rat. PLoS One, 5, e14071.

Aoki, E., Semba, R., Keino, H., Kato, K. and Kashiwamata, S. (1988) Glycinelike immunoreactivity in the rat auditory pathway. Brain Res, 442, 6371.

Assisi, C., Stopfer, M., Laurent, G. and Bazhenov, M. (2007) Adaptive regulation of sparseness by feedforward inhibition. Nat Neurosci, 10, 1176-1184.

Attneave, F. (1954) Some informational aspects of visual perception. Psychological review, 61, 183-193.

Ayala, Y.A. and Malmierca, M.S. (2013) Stimulus-specific adaptation and deviance detection in the inferior colliculus. Front Neural Circuits, 6, 89.

Ayala, Y.A., Perez-Gonzalez, D., Duque, D., Nelken, I. and Malmierca, M.S. (2013) Frequency discrimination and stimulus deviance in the inferior colliculus and cochlear nucleus. Front Neural Circuits, 6, 119.

Barlow, H. B. (1961) Possible principles underlying the transformation of sensory messages. In 'Sensory Communication' (ed. Rosenbluth, W. A.) 217-234 (MIT Press, Cambridge). 
Bartlett, E.L. and Smith, P.H. (1999) Anatomic, intrinsic, and synaptic properties of dorsal and ventral division neurons in rat medial geniculate body. J Neurophysiol, 81, 1999-2016.

Bartlett, E.L. and Wang, X. (2007) Neural representations of temporally modulated signals in the auditory thalamus of awake primates. J Neurophysiol, 97, 1005-1017.

Bäuerle, P., von der Behrens, W., Kossl, M. and Gaese, B.H. (2011) Stimulusspecific adaptation in the gerbil primary auditory thalamus is the result of a fast frequency-specific habituation and is regulated by the corticofugal system. J Neurosci, 31, 9708-9722.

Bendixen, A., SanMiguel, I. and Schroger, E. (2012) Early electrophysiological indicators for predictive processing in audition: a review. Int $J$ Psychophysiol, 83, 120-131.

Bregman, A.S. (1990). Auditory Scene Analysis: The Perceptual Organization of Sound. Cambridge, Massachusetts: The MIT Press.

Brenner, N., Bialek, W. and de Ruyter van Steveninck, R. (2000) Adaptive rescaling maximizes information transmission. Neuron, 26, 695-702.

Briley, P.M. and Krumbholz, K. (2013) The specificity of stimulus-specific adaptation in human auditory cortex increases with repeated exposure to the adapting stimulus. J Neurophysiol, 110, 2679-2688.

Buran, B.N., von Trapp, G. and Sanes, D.H. (2014) Behaviorally gated reduction of spontaneous discharge can improve detection thresholds in auditory cortex. $J$ Neurosci, 34, 4076-4081.

Cant, N.B. and Benson, C.G. (2003) Parallel auditory pathways: projection patterns of the different neuronal populations in the dorsal and ventral cochlear nuclei. Brain Res Bull, 60:457-474. 
Calford, M.B. (1983) The parcellation of the medial geniculate body of the cat defined by the auditory response properties of single units. J Neurosci, 3, 2350-2364.

Calford, M.B. and Aitkin, L.M. (1983) Ascending projections to the medial geniculate body of the cat: evidence for multiple, parallel auditory pathways through thalamus. J Neurosci, 3, 2365-2380.

Castro-Alamancos, M.A. (2004) Absence of rapid sensory adaptation in neocortex during information processing states. Neuron, 41, 455-464.

Castillo, P.E., Younts, T.J., Chavez, A.E. and Hashimotodani, Y. (2012) Endocannabinoid signaling and synaptic function. Neuron 76:70-81.

Clement, E.A., Richard, A., Thwaites, M., Ailon, J., Peters, S. and Dickson, C.T. (2008) Cyclic and sleep-like spontaneous alternations of brain state under urethane anaesthesia. PLoS One, 3, e2004.

Crick, F. (1984) Function of the thalamic reticular complex: the searchlight hypothesis. Proc Natl Acad Sci U S A, 81, 4586-4590.

Chelaru, M.I. and Dragoi, V. (2008) Efficient coding in heterogeneous neuronal populations. Proc Natl Acad Sci U S A, 105, 16344-16349.

Cherry, E. C. (1953). Some Experiments on the Recognition of Speech, with One and with Two Ears. The Journal of the Acoustical Society of America 25 (5): 975-79.

Chung, S., Li, X. and Nelson, S.B. (2002) Short-term depression at thalamocortical synapses contributes to rapid adaptation of cortical sensory responses in vivo. Neuron, 34, 437-446.

Dahmen, J.C., Keating, P., Nodal, F.R., Schulz, A.L. and King, A.J. (2010) Adaptation to stimulus statistics in the perception and neural representation of auditory space. Neuron, 66, 937-948. 
de Ribaupierre, F. (1997). Acoustical information processing in the auditory thalamus and cerebral cortex. In 'Anatomical and functional aspects of the cochlear nucleus' (Ehret, G., and Romand, R., Eds.). pp. 317-397. Oxford Univerity Press. Oxford.

Dean, I., Harper, N.S. and McAlpine, D. (2005) Neural population coding of sound level adapts to stimulus statistics. Nat Neurosci, 8, 1684-1689.

Doron, N.N. and Ledoux, J.E. (1999) Organization of projections to the lateral amygdala from auditory and visual areas of the thalamus in the rat. $J$ Comp Neurol, 412, 383-409.

Duque, D. and Malmierca, M.S. (2014) Stimulus-specific adaptation in the inferior colliculus of the mouse: anesthesia and spontaneous activity effects. Brain Struct Funct. Epub ahead of print.

Duque, D., Malmierca, M.S. and Caspary, D.M. (2014) Modulation of stimulus-specific adaptation by GABAA receptor activation or blockade in the medial geniculate body of the anaesthetized rat. J Physiol, 592, 729-743.

Duque, D., Perez-Gonzalez, D., Ayala, Y.A., Palmer, A.R. and Malmierca, M.S. (2012) Topographic distribution, frequency, and intensity dependence of stimulus-specific adaptation in the inferior colliculus of the rat. $J$ Neurosci, 32, 17762-17774.

Escera, C. and Malmierca, M.S. (2013) The auditory novelty system: An attempt to integrate human and animal research. Psychophysiology, 51, 111-123.

Eytan, D., Brenner, N. and Marom, S. (2003) Selective adaptation in networks of cortical neurons. J Neurosci, 23, 9349-9356. 
Fairhall, A.L., Lewen, G.D., Bialek, W. and de Ruyter Van Steveninck, R.R. (2001) Efficiency and ambiguity in an adaptive neural code. Nature, 412, 787-792.

Farley, B.J., Quirk, M.C., Doherty, J.J. and Christian, E.P. (2010) Stimulusspecific adaptation in auditory cortex is an NMDA-independent process distinct from the sensory novelty encoded by the mismatch negativity. J Neurosci, 30, 16475-16484.

Faye-Lund, H. and Osen, K.K. (1985) Anatomy of the inferior colliculus in rat. Anat Embryol (Berl), 171, 1-20.

Fishman, Y.I. (2013) The Mechanisms and Meaning of the Mismatch Negativity. Brain Topogr. 27(4):500-26.

Fishman, Y.I. and Steinschneider, M. (2010) Neural correlates of auditory scene analysis based on inharmonicity in monkey primary auditory cortex. J Neurosci, 30, 12480-12494.

Fishman, Y.I. and Steinschneider, M. (2012) Searching for the mismatch negativity in primary auditory cortex of the awake monkey: deviance detection or stimulus specific adaptation? J Neurosci, 32, 1574715758.

Freund, T.F., Katona, I. and Piomelli, D. (2003) Role of endogenous cannabinoids in synaptic signaling. Physiol. Rev. 83, 1017-1066.

Friauf, E., Hammerschmidt, B. and Kirsch, J. (1997) Development of adulttype inhibitory glycine receptors in the central auditory system of rats. J Comp Neurol, 385, 117-134.

Friston, K. (2005) A theory of cortical responses. Philos Trans R Soc Lond B Biol Sci. Apr 29;360(1456):815-36. 
Fritz, J.B., Elhilali, M., David, S.V. and Shamma, S.A. (2007) Auditory attention--focusing the searchlight on sound. Curr Opin Neurobiol, 17, 437-455.

Grill-Spector, K., Henson, R. and Martin, A. (2006) Repetition and the brain: neural models of stimulus-specific effects. Trends Cogn Sci, 10, 14-23.

Grimsley, C.A., Sanchez, J.T. and Sivaramakrishnan, S. (2013) Midbrain local circuits shape sound intensity codes. Front Neural Circuits 7:174.

Hari, R., Hamalainen, M., Ilmoniemi, R., Kaukoranta, E., Reinikainen, K., Salminen, J., Alho, K., Naatanen, R. and Sams, M. (1984) Responses of the primary auditory cortex to pitch changes in a sequence of tone pips: neuromagnetic recordings in man. Neurosci Lett, 50, 127-132.

Harris, R.M. (1987) Axon collaterals in the thalamic reticular nucleus from thalamocortical neurons of the rat ventrobasal thalamus. J Comp Neurol, 258, 397-406.

Hernandez, O., Espinosa, N., Perez-Gonzalez, D. and Malmierca, M.S. (2005) The inferior colliculus of the rat: a quantitative analysis of monaural frequency response areas. Neuroscience, 132, 203-217.

Hershenhoren, I., Taaseh, N., Antunes, F.M. and Nelken, I. (2014) Intracellular correlates of stimulus-specific adaptation. J Neurosci, 34, 3303-3319.

$\mathrm{Hu}, \mathrm{B}$. (2003) Functional organization of lemniscal and nonlemniscal auditory thalamus. Exp Brain Res, 153, 543-549.

Hu, B., Senatorov, V. and Mooney, D. (1994) Lemniscal and non-lemniscal synaptic transmission in rat auditory thalamus. J Physiol, 479 (Pt 2), 217-231. 
Huffman, R.F. and Henson, O.W., Jr. (1990) The descending auditory pathway and acousticomotor systems: connections with the inferior colliculus. Brain Res Brain Res Rev, 15, 295-323.

Isaacson, J.S. and Scanziani, M. (2011) How inhibition shapes cortical activity. Neuron, 72, 231-243.

Ito, T., Bishop, D.C. and Oliver, D.L. (2011) Expression of glutamate and inhibitory amino acid vesicular transporters in the rodent auditory brainstem. J Comp Neurol, 519, 316-340.

Jääskeläinen, I.P., Ahveninen, J., Bonmassar, G., Dale, A.M., Ilmoniemi, R.J., Levanen, S., Lin, F.H., May, P., Melcher, J., Stufflebeam, S., Tiitinen, H. and Belliveau, J.W. (2004) Human posterior auditory cortex gates novel sounds to consciousness. Proc Natl Acad Sci U S A, 101, 68096814.

Jacobsen, T., Horenkamp, T. and Schröger, E. (2003) Preattentive memorybased comparison of sound intensity. Audiol Neurootol, 8, 338-346.

Jones, E.G. (1975) Some aspects of the organization of the thalamic reticular complex. J Comp Neurol, 162, 285-308.

Kano, M., Ohno-Shosaku, T., Hashimotodani, Y., Uchigashima, M. and Watanabe, M. (2009) Endocannabinoid-mediated control of synaptic transmission. Physiol. Rev. 89, 309-380.

Kant, I. Crítica de la razón pura. Trad. de Pedro Ribas. Madrid, AlfaguaraSantillana, 1997 (13a ed.). ISBN 84-204-0407-1.

Katz, Y., Heiss, J.E. and Lampl, I. (2006) Cross-whisker adaptation of neurons in the rat barrel cortex. J Neurosci, 26, 13363-13372. 
Kern, A., Heid, C., Steeb, W.H., Stoop, N. and Stoop, R. (2008) Biophysical parameters modification could overcome essential hearing gaps. PLOS Comput Biol, 4, e1000161.

Klein, C., von der Behrens, W. and Gaese, B.H. (2014) Stimulus-Specific Adaptation in Field Potentials and Neuronal Responses to FrequencyModulated Tones in the Primary Auditory Cortex. Brain Topogr. 27(4):599-610.

Klug, A. and Grothe, B. (2010) Ethological stimuli. In: Rees, A. and Palmer, A.R. (eds) Oxford Handbook of Auditory Science: The Auditory Brain. Oxford University Press, ISBN 978-0-19-923328-1, pp. 171-190

Komura, Y., Tamura, R., Uwano, T., Nishijo, H., Kaga, K. and Ono, T. (2001) Retrospective and prospective coding for predicted reward in the sensory thalamus. Nature 412:546-549.

Komura, Y., Tamura, R., Uwano, T., Nishijo, H. and Ono, T. (2005) Auditory thalamus integrates visual inputs into behavioral gains. Nat. Neurosci. 8: 1203-1209.

Kostal, L., Lansky, P. and Rospars, J.P. (2008) Efficient olfactory coding in the pheromone receptor neuron of a moth. PLoS Comput Biol, 4, e1000053.

Laughlin, S. (1981) A simple coding procedure enhances a neuron's information capacity. Zeitschrift fur Naturforschung. Section C: Biosciences, 36, 910-912.

LeBeau, F.E., Malmierca, M.S. and Rees, A. (2001) lontophoresis in vivo demonstrates a key role for $\operatorname{GABA}(A)$ and glycinergic inhibition in shaping frequency response areas in the inferior colliculus of guinea pig. J Neurosci, 21, 7303-7312. 
LeBeau, F.E., Rees, A. and Malmierca, M.S. (1996) Contribution of GABA- and glycine-mediated inhibition to the monaural temporal response properties of neurons in the inferior colliculus. J Neurophysiol, 75, 902919.

Lee, C.C. and Sherman, S.M. (2010) Topography and physiology of ascending streams in the auditory tectothalamic pathway. Proc Natl Acad Sci U S A, 107, 372-377.

Lee, C.C. and Sherman, S.M. (2011) On the classification of pathways in the auditory midbrain, thalamus, and cortex. Hear Res, 276, 79-87.

Lee, C.C. and Winer, J.A. (2005) Principles governing auditory cortex connections. Cereb Cortex, 15, 1804-1814.

Lewicki, M.S. (2002) Efficient coding of natural sounds. Nat Neurosci, 5, 356363.

Li, Y., Evans, M.S. and Faingold, C.L. (1998) In vitro electrophysiology of neurons in subnuclei of rat inferior colliculus. Hear Res, 121, 1-10.

Liu, X., Yan, Y., Wang, Y. and Yan, J. (2010) Corticofugal modulation of initial neural processing of sound information from the ipsilateral ear in the mouse. PLoS One. 5: e14038.

Liberman, M.C. (1978) Auditory-nerve response from cats raised in a lownoise chamber. J Acoust Soc Am, 63, 442-455.

Llano, D.A. and Sherman, S.M. (2008) Evidence for nonreciprocal organization of the mouse auditory thalamocortical-corticothalamic projection systems. J. Comp Neurol. 507: 1209-1227.

Loftus, W.C., Malmierca, M.S., Bishop, D.C. and Oliver, D.L. (2008) The cytoarchitecture of the inferior colliculus revisited: a common 
organization of the lateral cortex in rat and cat. Neuroscience, 154, 196-205.

Lumani, A. and Zhang, H. (2010) Responses of neurons in the rat's dorsal cortex of the inferior colliculus to monaural tone bursts. Brain Res, 1351, 115-129.

Luo, F., Wang, Q., Kashani, A. and Yan, J. (2008) Corticofugal modulation of initial sound processing in the brain. J. Neurosci. 28: 11615-11621.

Maess, B., Jacobsen, T., Schroger, E. and Friederici, A.D. (2007) Localizing preattentive auditory memory-based comparison: magnetic mismatch negativity to pitch change. Neurolmage, 37, 561-571.

Malmierca, M.S. (2003) The structure and physiology of the rat auditory system: an overview. Int Rev Neurobiol, 56, 147-211.

Malmierca, M. S., and Hackett, T. A. (2010). Structural organization of the ascending auditory pathway. In: Rees, A. and Palmer, A.R. (eds) The Oxford Handbook of Auditory Science: The Auditory Brain. Oxford University Press, ISBN 978-0-19-923328-1, pp. 9-41

Malmierca, M.S., Blackstad, T.W. and Osen, K.K. (2011) Computer-assisted 3$D$ reconstructions of Golgi-impregnated neurons in the cortical regions of the inferior colliculus of rat. Hear Res, 274, 13-26.

Malmierca, M.S., Saint Marie, R.L., Merchán, M.A. and Oliver, D.L. (2005) Laminar inputs from dorsal cochlear nucleus and ventral cochlear nucleus to the central nucleus of the inferior colliculus: two patterns of convergence. Neuroscience, 136:883-894.

Malmierca, M.S., Blackstad, T.W., Osen, K.K., Karagulle, T. and Molowny, R.L. (1993) The central nucleus of the inferior colliculus in rat: a Golgi and computer reconstruction study of neuronal and laminar structure. $J$ Comp Neurol, 333, 1-27. 
Malmierca, M.S., Cristaudo, S., Perez-Gonzalez, D. and Covey, E. (2009) Stimulus-specific adaptation in the inferior colliculus of the anesthetized rat. J Neurosci, 29, 5483-5493.

Malmierca, M.S., Hernandez, O., Falconi, A., Lopez-Poveda, E.A., Merchan, M. and Rees, A. (2003) The commissure of the inferior colliculus shapes frequency response areas in rat: an in vivo study using reversible blockade with microinjection of kynurenic acid. Exp Brain Res, 153, 522-529.

Malmierca, M.S., Merchán, M.A., Henkel, C.K. and Oliver, D.L. (2002) Direct projections from cochlear nuclear complex to auditory thalamus in the rat. J Neurosci, 22, 10891-10897.

Malmierca, M.S., Sanchez-Vives, M.V., Escera, C. and Bendixen, A. (2014) Neuronal adaptation, novelty detection and regularity encoding in audition. Front Syst Neurosci. 8:111.

Malmierca, M.S., Seip, K.L. and Osen, K.K. (1995) Morphological classification and identification of neurons in the inferior colliculus: a multivariate analysis. Anat Embryol (Berl), 191, 343-350.

Maravall, M., Petersen, R.S., Fairhall, A.L., Arabzadeh, E. and Diamond, M.E. (2007) Shifts in coding properties and maintenance of information transmission during adaptation in barrel cortex. PLOS Biol, 5, e19.

May, P.J. and Tiitinen, H. (2010) Mismatch negativity (MMN), the devianceelicited auditory deflection, explained. Psychophysiology, 47, 66-122.

Merchán, M., Aguilar, L.A., Lopez-Poveda, E.A. and Malmierca, M.S. (2005) The inferior colliculus of the rat: quantitative immunocytochemical study of GABA and glycine. Neuroscience, 136, 907-925. 
Mesgarani, N. and Chang, E.F. (2012) Selective cortical representation of attended speaker in multi-talker speech perception. Nature, 485, 233236.

Mill, R., Coath, M., Wennekers, T. and Denham, S.L. (2011a) Abstract Stimulus-Specific Adaptation Models. Neural Comput. 23(2):435-76.

Mill, R., Coath, M., Wennekers, T. and Denham, S.L. (2011b) A neurocomputational model of stimulus-specific adaptation to oddball and Markov sequences. PLoS Comput Biol, 7, e1002117.

Miller, E.K., Nieder, A., Freedman, D.J. and Wallis, J.D. (2003) Neural correlates of categories and concepts. Curr Opin Neurobiol, 13, 198203.

Näätänen, R., Gaillard, A.W. and Mantysalo, S. (1978) Early selectiveattention effect on evoked potential reinterpreted. Acta Psychol (Amst), 42, 313-329.

Näätänen, R., Pääviläinen, P., Tiitinen, H., Jiang, D. and Alho, K. (1993) Attention and mismatch negativity. Psychophysiology, 30, 436-450.

Näätänen, R., Tervaniemi, M., Sussman, E., Pääviläinen, P. and Winkler, I. (2001) "Primitive intelligence" in the auditory cortex. Trends Neurosci, 24, 283-288.

Nelken, I. (2014) Stimulus-specific adaptation and deviance detection in the auditory system: experiments and models. Biol Cybern. Epub ahead of print.

Nelken, I. and Ulanovsky, N. (2007) Mismatch Negativity and StimulusSpecific Adaptation in Animal Models. Journal of Psychophysiology, 21, 214-223. 
Nir, Y., Vyazovskiy, V.V., Cirelli, C., Banks, M.I. and Tononi, G. (2013) Auditory Responses and Stimulus-Specific Adaptation in Rat Auditory Cortex are Preserved Across NREM and REM Sleep. Cereb Cortex. Epub ahead of print.

Pagliardini, S., Funk, G.D. and Dickson, C.T. (2013) Breathing and brain state: urethane anesthesia as a model for natural sleep. Respiratory physiology and neurobiology, 188, 324-332.

Pagliardini, S., Greer, J.J., Funk, G.D. and Dickson, C.T. (2012) Statedependent modulation of breathing in urethane-anesthetized rats. $J$ Neurosci, 32, 11259-11270.

Palmer, A.R., Shackleton, T.M., Sumner, C.J., Zobay, O. and Rees, A. (2013) Classification of frequency response areas in the inferior colliculus reveals continua not discrete classes. J Physiol, 591, 4003-4025.

Patel, C.R., Redhead, C., Cervi, A.L. and Zhang, H. (2012) Neural sensitivity to novel sounds in the rat's dorsal cortex of the inferior colliculus as revealed by evoked local field potentials. Hear Res, 286, 41-54.

Penzo, M.A. and Peña, J.L. (2009) Endocannabinoid-mediated long-term depression in the avian midbrain expressed presynaptically and postsynaptically. J Neurosci, 29:4131-4139.

Pérez-González, D., Hernandez, O., Covey, E. and Malmierca, M.S. (2012) GABA(A)-Mediated Inhibition Modulates Stimulus-Specific Adaptation in the Inferior Colliculus. PLoS One, 7, e34297.

Pérez-González, D. and Malmierca, M.S. (2012) Variability of the time course of stimulus-specific adaptation in the inferior colliculus. Front Neural Circuits, 6, 107. 
Pérez-González, D., Malmierca, M.S. and Covey, E. (2005) Novelty detector neurons in the mammalian auditory midbrain. Eur J Neurosci, 22, 2879-2885.

Peruzzi, D., Bartlett, E., Smith, P.H. and Oliver, D.L. (1997) A monosynaptic GABAergic input from the inferior colliculus to the medial geniculate body in rat. J Neurosci, 17, 3766-3777.

Polley, D.B., Read, H.L., Storace, D.A. and Merzenich, M.M. (2007) Multiparametric auditory receptive field organization across five cortical fields in the albino rat. J Neurophysiol, 97, 3621-3638.

Ponnath, A., Hoke, K.L. and Farris, H.E. (2013) Stimulus change detection in phasic auditory units in the frog midbrain: frequency and ear specific adaptation. J Comp Physiol A Neuroethol Sens Neural Behav Physiol.

Reches, A. and Gutfreund, Y. (2008) Stimulus-specific adaptations in the gaze control system of the barn owl. J Neurosci, 28, 1523-1533.

Reches, A., Netser, S. and Gutfreund, Y. (2010) Interactions between stimulus-specific adaptation and visual auditory integration in the forebrain of the barn owl. J Neurosci, 30, 6991-6998.

Rechtschaffen, A., Hauri, P. and Zeitlin, M. (1966) Auditory awakening thresholds in REM and NREM sleep stages. Perceptual and motor skills, 22, 927-942.

Rees, A., Sarbaz, A., Malmierca, M.S. and Le Beau, F.E. (1997) Regularity of firing of neurons in the inferior colliculus. J Neurophysiol, 77, 29452965.

Regehr, W.G., Carey, M.R., and Best, A.R. (2009) Activity-dependent regulation of synapses by retrograde messengers. Neuron, 63, 154170. 
Richardson, B.D., Hancock, K.E. and Caspary, D.M. (2013) Stimulus-Specific Adaptation in Auditory Thalamus of Young and Aged Awake Rats. J Neurophysiol. 110(8):1892-902.

Robinson, B.L. and McAlpine, D. (2009) Gain control mechanisms in the auditory pathway. Curr Opin Neurobiol, 19, 402-407.

Rose, D. and Blakemore, C. (1974) Effects of bicuculline on functions of inhibition in visual cortex. Nature, 249, 375-377.

Rothman, J.S., Cathala, L., Steuber, V. and Silver, R.A. (2009) Synaptic depression enables neuronal gain control. Nature 457: 1015-1018.

Rouiller, E.M., Colomb, E., Capt, M. and De Ribaupierre, F. (1985) Projections of the reticular complex of the thalamus onto physiologically characterized regions of the medial geniculate body. Neurosci Lett, 53, 227-232.

Rouiller, E.M., Rodrigues-Dagaeff, C., Simm, G., De Ribaupierre, Y., Villa, A. and De Ribaupierre, F. (1989) Functional organization of the medial division of the medial geniculate body of the cat: tonotopic organization, spatial distribution of response properties and cortical connections. Hear Res, 39, 127-142.

Rudolph, U. and Antkowiak, B. (2004) Molecular and neuronal substrates for general anaesthetics. Nat Rev Neurosci, 5, 709-720.

Saldaña, E., Feliciano, M. and Mugnaini, E. (1996) Distribution of descending projections from primary auditory neocortex to inferior colliculus mimics the topography of intracollicular projections. J Comp Neurol, 371, 15-40.

Saldaña, E. and Merchán, M.A. (1992) Intrinsic and commissural connections of the rat inferior colliculus. J Comp Neurol, 319, 417-437. 
Schofield, B.R. (2010) Projections from auditory cortex to midbrain cholinergic neurons that project to the inferior colliculus. Neuroscience, 166, 231-240.

Schofield, B.R., Mellott, J.G. and Motts, S.D. (2014) Subcollicular projections to the auditory thalamus and collateral projections to the inferior colliculus. Front Neuroanat, 8, 70.

Scholl, B., Gao, X. and Wehr, M. (2008) Level dependence of contextual modulation in auditory cortex. J Neurophysiol, 99, 1616-1627.

Schopenhauer, A. El mundo como voluntad y representación. Volumen I (2009) 2a edición, Madrid, Trotta (2 tomos) ISBN 978-84-9879-078-8.

Schröger, E. and Wolff, C. (1998) Attentional orienting and reorienting is indicated by human event-related brain potentials. Neuroreport, 9, 3355-3358.

Shannon, C.E. (1948) A mathematical theory of communication. Bell System Technical Journal 27, 379-423 and 623-656.

Sherman, S.M. (2007) The thalamus is more than just a relay. Curr. Opin. Neurobiol. 17:417-422.

Shneiderman, A. and Henkel, C.K. (1987) Banding of lateral superior olivary nucleus afferents in the inferior colliculus: a possible substrate for sensory integration. J Comp Neurol, 266:519-534.

Simpson, A.J., Harper, N.S., Reiss, J.D. and McAlpine, D. (2014) Selective adaptation to "oddball" sounds by the human auditory system. J Neurosci, 34, 1963-1969.

Sivaramakrishnan, S., Sterbing-D'Angelo, S.J., Filipovic, B., D'Angelo, W.R., Oliver, D.L. and Kuwada, S. (2004) GABA(A) synapses shape neuronal 
responses to sound intensity in the inferior colliculus. I Neurosci, 24:5031-5043.

Smith, P.H. and Spirou, G.A. (2002) From the cochlea to the cortex and back. In 'Integrative functions in the mammalian auditory pathway. Springer handbook of auditory research' (Oertel D, Fay, RR and Popper AN Eds.). pp. 6-71. Springer. New York.

Smirnakis, S.M., Berry, M.J., Warland, D.K., Bialek, W. and Meister, M. (1997) Adaptation of retinal processing to image contrast and spatial scale. Nature, 386, 69-73.

Szymanski, F.D., Garcia-Lazaro, J.A. and Schnupp, J.W. (2009) Current source density profiles of stimulus-specific adaptation in rat auditory cortex. $J$ Neurophysiol, 102, 1483-1490.

Taaseh, N., Yaron, A. and Nelken, I. (2011) Stimulus-specific adaptation and deviance detection in the rat auditory cortex. PLoS One, 6, e23369.

Thomas, J.M., Morse, C., Kishline, L., O'Brien-Lambert, A., Simonton, A., Miller, K.E. and Covey, E. (2012) Stimulus-specific adaptation in specialized neurons in the inferior colliculus of the big brown bat, Eptesicus fuscus. Hear Res, 291, 34-40.

Tiitinen, H., May, P., Reinikainen, K. and Näätänen, R. (1994) Attentive novelty detection in humans is governed by pre-attentive sensory memory. Nature, 372, 90-92.

Trattner, B., Berner, S., Grothe, B. and Kunz, L. (2013) Depolarization-induced suppression of a glycinergic synapse in the superior olivary complex by endocannabinoids. J Neurochemistry 127:78-90.

Ulanovsky, N., Las, L., Farkas, D. and Nelken, I. (2004) Multiple time scales of adaptation in auditory cortex neurons. J Neurosci, 24, 10440-10453. 
Ulanovsky, N., Las, L. and Nelken, I. (2003) Processing of low-probability sounds by cortical neurons. Nat Neurosci, 6, 391-398.

Varela, J.A., Sen, K., Gibson, J., Fost, J., Abbott, L.F. and Nelson, S.B. (1997) A quantitative description of short-term plasticity at excitatory synapses in layer 2/3 of rat primary visual cortex. J Neurosci, 17: 7926-7940.

von Békésy G (1960) Experiments in hearing. New York: Mc Graw-Hill.

von der Behrens, W., Bauerle, P., Kossl, M. and Gaese, B.H. (2009) Correlating stimulus-specific adaptation of cortical neurons and local field potentials in the awake rat. J Neurosci, 29, 13837-13849.

Wark, B., Lundstrom, B.N. and Fairhall, A. (2007) Sensory adaptation. Curr Opin Neurobiol, 17, 423-429.

Westerman, L.A. and Smith, R.L. (1985) Rapid adaptation depends on the characteristic frequency of auditory nerve fibers. Hear res, 17:197-198.

Willard, F.H., and Ryugo, D.K. (1983) Anatomy of the central auditory system. In: The Auditory Psychobiology of the Mouse, (ed., J.F. Willott). Charles C Thomas Publisher, Springfield, IL, pp. 201-304.

Wilson, R.I. and Nicoll, R.A. (2001) Endogenous cannabinoids mediate retrograde signalling at hippocampal synapses. Nature, 410, 588-592.

Winer, J.A. (1985) The medial geniculate body of the cat. Advances in anatomy, embryology, and cell biology, 86, 1-97.

Winer, J.A. (2006) Decoding the auditory corticofugal systems. Hear Res, 212, $1-8$.

Winer, J.A. and Larue, D.T. (1987) Patterns of reciprocity in auditory thalamocortical and corticothalamic connections: study with horseradish peroxidase and autoradiographic methods in the rat medial geniculate body. J Comp Neurol, 257, 282-315. 
Winer, J.A. and Larue, D.T. (1996) Evolution of GABAergic circuitry in the mammalian medial geniculate body. Proc Natl Acad Sci U S A, 93, 3083-3087.

Winer, J.A., Kelly, J.B. Larue, D.T. (1999) Neural architecture of the rat medial geniculate body. Hear. Res. 130: 19-41.

Winer, J.A., Saint Marie, R.L., Larue, D.T. and Oliver, D.L. (1996) GABAergic feedforward projections from the inferior colliculus to the medial geniculate body. Proc Natl Acad Sci U S A, 93, 8005-8010.

Winkler, I., Denham, S.L. and Nelken, I. (2009) Modeling the auditory scene: predictive regularity representations and perceptual objects. Trends Cogn Sci, 13, 532-540.

Xu, X., Yu, X., He, J. and Nelken, I. (2014) Across-ear stimulus-specific adaptation in the auditory cortex. Front Neural Circuits, 8, 89.

Yaron, A., Hershenhoren, I. and Nelken, I. (2012) Sensitivity to complex statistical regularities in rat auditory cortex. Neuron, 76, 603-615.

Yu, X.J., Xu, X.X., He, S. and He, J. (2009) Change detection by thalamic reticular neurons. Nat Neurosci, 12, 1165-1170.

Zhao, Y. and Tzounopoulos, T. (2011) Physiological activation of cholinergic inputs controls associative synaptic plasticity via modulation of endocannabinoid signaling. J Neurosci, 31:3158-3168.

Zhao, Y., Rubio, M.E. and Tzounopoulos, T. (2009) Distinct functional and anatomical architecture of the endocannabinoid system in the auditory brainstem. J Neurophysiol, 101:2434-2446.

Zhao, L., Liu, Y., Shen, L., Feng, L. and Hong, B. (2011) Stimulus-specific adaptation and its dynamics in the inferior colliculus of rat. Neuroscience. 181:163-74. 
ANNEX: SPANISH SUMMARY 



\section{Introducción}

El mundo que nos rodea es una realidad que no puede ser percibida en su totalidad, aunque para los seres vivos esa realidad es irrelevante: los sistemas sensoriales simplemente tienen que ofrecer una representación mínima del medio ambiente, para así proveer a los organismos de estímulos lo suficientemente simples para que éstos generen respuestas óptimas para la supervivencia (Klug y Grothe, 2010).

Codificación de las señales por el cerebro

La rápida adopción de la teoría de la información (Shannon, 1948) al campo de la neurobiología teórica y experimental (Attneave, 1954; Barlow, 1961) implicó un brusco cambio de paradigma a la hora de plantearse de qué manera el cerebro está codificando el mundo que nos rodea. La hipótesis de la codificación eficiente planteada por Barlow (1961) implicaba tres principios básicos: 1) los sistemas sensoriales son capaces de detectar patrones relevantes de entre el continuo flujo de señales que reciben, 2) los diferentes núcleos sensoriales actúan como filtros modificables y 3) para percibir adecuadamente los mensajes del mundo exterior es necesario eliminar toda la información redundante. La reducción de toda la información reiterativa puede llevarse a cabo mediante la adaptación: si un sistema sensorial es capaz de ajustar su estrategia de codificación de señales, la adaptación ayudaría a dicho sistema a codificar eficientemente los estímulos naturales (Wark y col., 2007).

Durante las últimas décadas varios artículos han aportado pruebas irrefutables de que los sistemas visual (Laighlin, 1981; Smirnakis y col., 1997; Fairhall y col., 2001), auditivo (Lewicki, 2002; Ulanovsky y col., 2003; Dean y col., 2005; Dahmen y col., 2010), somatosensorial (Katz y col., 2006; Maravall y col., 2007) y olfativo (Assisi y col., 2007; Kostal y col., 2008) son capaces de 
codificar eficientemente las variaciones de estímulos que se encuentran en la naturaleza. Todos estos experimentos refuerzan la idea de que la adaptación es un proceso activo que permite a los animales tener una óptima representación del mundo que los rodea. De entre todos los sistemas sensoriales, el auditivo es particularmente interesante. El ambiente auditivo natural está lleno de sonidos, muchos de los cuales pueden tener un significado evolutivo, entendiéndose como esencial para la supervivencia, y muchos otros que no tienen por qué afectar la propia integridad.

\section{El sistema auditivo}

El sistema auditivo central consiste en una sucesión de núcleos auditivos interconectados entre ellos, a través de los cuales es trasmitida toda la información auditiva recibida por el sistema periférico (Malmierca y Hacket, 2010). La información que proviene de la cóclea llega a los núcleos cocleares a través del nervio auditivo manteniendo la tonotopía que se ha formado en la cóclea (von Bèkèsy, 1960), es decir, conservando una organización segregada de bajas a altas frecuencias. Los núcleos cocleares distribuyen toda la información a núcleos posteriores mediante tres vías paralelas de procesamiento que convergen finalmente en el colículo inferior (Malmierca, 2003).

El colículo inferior (IC según las siglas de la nomenclatura anglosajona, inferior colliculus) es de los centros más importantes de la vía auditiva ya que reconstruye toda la información descendente proveniente de los núcleos cocleares, el complejo olivar superior y los núcleos del lemnisco lateral (Malmierca y Hacket, 2010). Anatómicamente se divide en cuatro regiones: un núcleo central y tres cortezas: lateral, rostral y dorsal (Loftus y col., 2008). El núcleo central recolecta todas las fibras aferentes derivadas del tronco cerebral y está organizado en láminas fibrodendríticas que mantienen la tonotopía, procesando las frecuencias altas en la región 
ventrolateral y las frecuencias bajas en la dorsomedial (Faye-Lund y Osen, 1985; Malmierca y col., 1993). Por otro lado, las cortezas lateral, rostral y dorsal del IC parecen tener una función diferente, siendo de gran interés de estudio debido a que estas regiones reciben masivas proyecciones corticofugas (Saldaña y col., 1996) y presentan interesantes respuestas multisensoriales (Aitlkin y col., 1978, 1981). Las neuronas localizadas en la cortezas del IC presentan un alto grado de desorganización dendrítica (Malmierca, 1991), al contrario de los árboles dendríticos de las neuronas del núcleo central. Esta teórica desorganización permitiría un gran nivel de integración, facilitando la adquisición de la información proveniente de la integración de distintas frecuencias (Loftus y col., 2008).

El cuerpo geniculado medial (MGB según las siglas de la nomenclatura anglosajona, medial geniculate body), en el tálamo auditivo, es la siguiente estación de la vía auditiva. Es un centro esencial de modulación de las señales auditivas (Sherman, 2007) y tiene importantes implicaciones en el procesamiento emocional de los sonidos (Doron y Leroux, 1999). Anatómicamente presenta tres divisiones (Winer, 1985). La división ventral está organizada tonotópicamente y sus neuronas responden eficientemente a los estímulos acústicos. Ambas divisiones medial y dorsal presentan una importante respuesta multisensorial (Smith y Spirou, 2002).

La distribución anatómica del IC y del MGB no es baladí y de ella surgen dos vías paralelas de procesamiento auditivo: la vía lemniscal y la nolemniscal (Hu y col., 1994; Hu, 2003). La vía lemniscal comienza en el núcleo central del IC y proyecta al núcleo ventral del MGB, que a su vez proyecta a la corteza auditiva primaria. La vía no-lemniscal comienza en las regiones corticales del IC, proyecta a las divisiones mediales y dorsales del MGB y este a su vez a la corteza auditiva secundaria (Hu y col., 1994; Hu, 2003; Lee y Sherman, 2010, 2011). La vía lemniscal provee a los organismos de una 
representación fiable de las características del sonido (de Ribaupierre, 1997), mientras que la no-lemniscal lleva básicamente información contextual (Calford y Aitkin, 1983), multisensorial (Hu y col, 1994) y emocional (Komura y col., 2001, 2005).

\section{Detección de la novedad auditiva}

De entre todos los sonidos generados continua y simultáneamente por diversas fuentes en un ambiente natural normal, el sistema auditivo tiene que seleccionar qué elementos acústicos son verdaderamente relevantes y crear constructos perceptuales (Fishman y Steinschneider, 2010) con el propósito de realizar adecuadamente un análisis de la escena auditiva (Bregman, 1990; Winkler y col., 2009). Una manera de organizar dicha escena acústica implica la retención de los objetos sonoros (Winkler y col., 2009), el almacenamiento de los objetos regulares en una huella mnemónica y la generación de predicciones sobre posibles futuros eventos al compararlos con esa huella retenida en la memoria (Bendixen y col., 2012). Este análisis de la escena auditiva es la aproximación teórica actual a la detección de la novedad auditiva (Näätänen y col., 1978). Para entenderlo se tiene que tener en cuenta que dentro de cualquier escena auditiva coexisten sonidos repetitivos, generalmente irrelevantes, con otros sonidos novedosos, raros, que pueden precisar de una respuesta inmediata de los animales. Como asimilar y responder a tal cantidad de información es una tarea imposible para el sistema nervioso, el procesamiento de todos estos sonidos tiene que ser compensado de alguna manera en función de su importancia relativa. En 1978, Näätänen y colaboradores, usando potenciales evocados auditivos, encontraron un componente cerebral que parecía reflejar la capacidad para detectar sonidos raros: el potencial de disparidad.

Los potenciales evocados auditivos son un conjunto de señales bioeléctricas generadas por el cerebro ante una estimulación auditiva. Esta 
estimulación provoca la activación de determinados conjuntos neuronales que, a su vez, originan una secuencia de ondas cerebrales. El potencial de disparidad (MMN según las siglas de la nomenclatura anglosajona, mismatch negativity) es uno de estos componentes (Näätänen y col., 1978; Tiitinen y col., 1994). El MMN es una onda con el pico de latencia entre los 150 y los 200 ms (Nelken, 2014) que se puede obtener mediante una multitud de protocolos experimentales, aunque el más común y usado históricamente es el paradigma oddball. Dicho paradigma consiste en la presentación de una secuencia de estímulos repetitivos (sonidos estándar) entre los cuales se intercala ocasionalmente un estímulo diferente (sonido raro). El MMN es la diferencia de señal entre las ondas obtenidas por el estímulo estándar y el raro. Aunque la atención puede modular la detección de la novedad (Näätänen y col., 1993), las bases fisiológicas de dicho proceso son claramente preatentivas (Tiitinen y col., 1994; Schröger, 1998). Actualmente existen dos teorías diferentes acerca de cuáles son los orígenes fenomenológicos del MMN (Fishman, 2013): 1) la codificación predictiva (Näätänen y col., 2001; Friston, 2005), dónde el MMN sería una señal de error y 2) la adaptación neuronal, en la cual el MMN sería solamente el reflejo de los procesos adaptativos que se suceden a lo largo de la vía auditiva, es decir, un artefacto de la sustracción (May y Tiitinen, 2010).

\section{Adaptación específica a estímulos}

De manera paralela a la que se sucedía este debate, se encontró un fenómeno neuronal interesante: la adaptación específica a estímulos (SSA según las siglas de la nomenclatura anglosajona, stimulus-specific adaptation), es decir, la habilidad de algunas neuronas auditivas de mantener una respuesta estable y continua a los sonidos raros mientras la respuesta a los sonidos repetidos se encuentra muy adaptada, hasta llegar el punto de poder desaparecer (Ulanovsky y col., 2003; Malmierca y col., 2009). 
La SSA fue inicialmente descrita en la corteza auditiva (Ulanovsky y col., 2003), aunque posteriormente también fue encontrada en el IC (PérezGonzález y col., 2005) y el MGB (Anderson y col., 2009). Hasta este momento, el IC es la primera estación de la vía auditiva donde se ha encontrado SSA, ya que experimentos en los núcleos cocleares se han mostrado infructuosos (Ayala y col., 2013). Las propiedades básicas de la SSA en el sistema auditivo se han estudiado en detalle tanto en el IC (para una revisión exhaustiva ver Ayala y Malmierca, 2013), el MGB (para una revisión exhaustiva ver Antunes y Malmierca, 2014) y la corteza auditiva (para una revisión exhaustiva ver Escera y Malmierca, 2013 o Nelken, 2014).

Se sabe que la SSA depende de algunos factores tales como el contraste de frecuencia (en octavas) entre el estímulo estándar y el raro, la probabilidad de ocurrencia del estímulo raro o el intervalo de tiempo entre estímulos consecutivos (Ulanovsky y col., 2003, Malmierca y col., 2009). También se sabe que las regiones no-lemniscales del IC y el MGB son las que presentan mayores niveles de SSA (Malmierca y col., 2009; Antunes y col., 2010), aunque los niveles de SSA encontrados en la región lemniscal de la corteza auditiva rompen con esta distribución (Ulanovsky y col., 2003). Teniendo en cuenta la masiva proyección descendente de la corteza auditiva a las regiones no-lemniscales de IC y de MGB, Nelken y colaboradores (2003) consideraron que la SSA se generaba en la corteza y se transmitía posteriormente a núcleos inferiores. Recientes experimentos han demostrado que la desactivación cortical no afecta a la SSA de las regiones no-lemniscales del MGB (Antunes y Malmierca, 2011) y del IC (Anderson y Malmierca, 2013). De esta manera, la SSA podría tratarse de un fenómeno que se origina en núcleos inferiores y se transmite posteriormente hasta la corteza auditiva, refinándose en cada estación intermedia. 


\section{Hipótesis}

Hasta ahora, los mecanismos neuronales que generan la SSA en el sistema auditivo siguen sin conocerse. Dos teorías diferentes se han desarrollado en los últimos años para intentar explicarlos. Por un lado, la SSA podría ser un mecanismo que operara en la salida de la neurona, al nivel del potencial de membrana (Abolafia y col., 2011), alterando la sensibilidad general de la neurona. Por otro lado, la SSA también podría ser un mecanismo que actuara a nivel de las aferentes neuronales (Ulanovsky y col., 2004), antes de que la neurona integrara las respuestas. Dicho modelo, que implica mecanismos como la depresión sináptica (Grill-Spector y col., 2006; Briley y Krumbholz, 2013), se ha destacado como la mejor explicación para la SSA (teoría de los canales de adaptación específicos de frecuencia: Eytan y col., 2003; Taaseh y col., 2011).

Además, el rol de la inhibición en la generación de la SSA no está claro. Según unos autores la inhibición puede modular activamente la SSA (Yu y col., 2009), mientras otros dicen que la inhibición por sí sola no la está generando (Pérez-González y col., 2012). Finalmente, cabe resaltar que la mayoría de los experimentos sobre la SSA se han realizado bajo anestesia, aún a sabiendas de que ésta modifica muchas propiedades neuronales al alterar el sensible equilibrio entre la inhibición y la excitación neuronal (Rudolph y Antkowiak, 2004). Sabiendo todo esto, nuestras hipótesis son:

1. La SSA es un mecanismo dependiente de las aferentes neuronales y definido por el rango de frecuencias que abarca cada aferente.

2. La SSA es un mecanismo auténtico y no un artefacto de la anestesia, aunque puede estar controlado por el sistema inhibitorio. 


\section{Objetivos}

Considerando 1) que la mayoría de los experimentos sobre la SSA consistían en una evaluación general de su sensibilidad sin tener en cuenta las regiones específicas de los campos neuronales, 2) que la sensibilidad a sonidos que sólo difieren en intensidad casi no está estudiada, 3) que las implicaciones de la inhibición en la SSA son confusas y 4) que la mayoría de los experimentos se han realizado en animales anestesiados, los objetivos de esta Tesis Doctoral son:

1. Evaluar si la SSA es un proceso dependiente de frecuencia e intensidad o si, por el contrario, presenta un valor único para cada neurona (Estudio I).

2. Comprobar si la SSA está generada o modulada por el sistema GABAérgico en el MGB, donde $\mathrm{GABA}$ es el único neurotransmisor inhibitorio (Estudio II).

3. Demostrar que la SSA no está afectada por la anestesia y entender si el estado de alerta de los animales puede modificar su sensibilidad (Estudio III).

4. Determinar si la SSA puede ser evocada por sonidos raros que difieren en intensidad (Estudio IV). 


\section{Resumen artículo I: SSA depende de frecuencia e intensidad}

\section{Objetivos}

Hasta la fecha, la mayoría de estudios sobre la SSA se habían centrado en el análisis de su respuesta teniendo en cuenta factores contextuales como la probabilidad de ocurrencia del estímulo raro o la frecuencia de repetición de los sonidos. Aun así, nadie se había planteado si la SSA variaba dentro del campo receptivo neuronal (FRA según las siglas de la nomenclatura anglosajona, frequency response area), es decir, se ignoraba si la SSA era una propiedad única de la neurona o, por el contrario, una característica dependiente de la intensidad, la frecuencia del sonido o el patrón de disparo de la neurona en esa zona determinada del FRA.

\section{Metodología}

De este modo, se usó el paradigma oddball para evaluar la SSA en 900 pares de frecuencias de 115 neuronas del IC de la rata (unas 8 parejas por neurona). La SSA fue cuantificada y comparada entre los diferentes pares de frecuencias dentro de cada FRA, teniendo en cuenta las intensidades y las frecuencias usadas en cada caso. También se evaluó si el tipo de FRA de la neurona o el patrón de disparo en esa región del FRA afectaban a la sensibilidad de la neurona para mostrar altos niveles de SSA. Para finalizar, se realizó un análisis exhaustivo de los niveles de SSA considerando las regiones que constituyen el IC.

\section{Resultados}

Nuestros resultados mostraron que la SSA es manifiestamente dependiente tanto de frecuencia como de intensidad, mostrando los niveles más elevados a altas frecuencias, a bajas intensidades y cuando las neuronas presentan un tipo de respuesta onset. La tendencia que mostraba altos niveles de SSA a altas frecuencias no estaba sesgada debido a una mayor 
cantidad de neuronas con buena respuesta a altas frecuencias, pues dicha tendencia también se mantenía dentro de cada neurona: los pares de frecuencias en la región de altas frecuencias de la neurona también mostraban mayores niveles de SSA. Además, confirmamos que la SSA es mucho más robusta en las regiones corticales (no-lemniscales) del IC y que hay una correlación positiva entre la SSA y el ancho de banda de los FRAs.

\section{Conclusiones}

Todo esto sugiere que las neuronas del IC de la rata muestran una mayor sensibilidad para discernir sonidos discretos y sutiles, sugiriendo que la respuesta a un sonido de alta intensidad siempre es relevante para el organismo. Estas neuronas también muestran mayores niveles de SSA para los sonidos de altas frecuencias, un rango de frecuencias que incluye una gran cantidad de sonidos relevantes para la supervivencia de estos animales. Además, la mayor capacidad integrativa de las neuronas de las regiones corticales del IC también se refleja en su sensibilidad para discernir sonidos: cuanto mayor es el rango de frecuencias que puede integrar una neurona, mayor es la capacidad para discriminar entre las frecuencias. Así pues, como la SSA en el IC no muestra un valor único neuronal no está mediada por propiedades intrínsecas de la membrana. Valorando todos estos datos, las explicaciones más plausibles para este fenómeno son 1) el equilibrio entre la excitación y la inhibición o 2) una posible segregación de las aferentes neuronales. 


\section{Resumen artículo II: El sistema GABAérgico modula la SSA}

\section{Objetivos}

Teniendo en cuenta los datos obtenidos en el artículo anterior, nos planteamos si realmente la relación que se establece en cada neurona entre la excitación y la inhibición era la desencadenante de la SSA. Así pues, como el sistema GABAérgico es conocido por regular diversas propiedades neuronales en el sistema auditivo, bloqueamos y/o activamos los receptores de GABA con el fin de observar qué efectos tiene dicho sistema sobre la sensibilidad neuronal a SSA. Los experimentos fueron realizados en el MGB porque en este núcleo GABA es el principal neurotransmisor inhibitorio, pues la glicina está prácticamente ausente.

\section{Metodología}

Registramos 52 neuronas extracelularmente con electrodos que tenían adheridos pipetas de vidrio rellenas de agonistas (GABA y gaboxadol) y/o antagonistas (gabazina) de los receptores de $G_{A B A}$. Al encontrar una neurona, evaluábamos sus niveles de SSA y luego eyectábamos las diferentes drogas para ver 1) qué efectos tenían sobre las propiedades básicas de la neurona y 2) si afectaban a la sensibilidad de la neurona para la SSA. Dicha eyección se realizaba mediante la técnica de la microiontoforesis, que consiste en retener las drogas manteniendo una corriente con polaridad inversa y eyectarlas por repulsión al poner una corriente con la misma polaridad. Al finalizar las evaluaciones con las drogas, siempre tratábamos de recuperar los valores obtenidos al principio como control de la integridad de la neurona.

\section{Resultados}

Nuestros resultados demostraron que tanto GABA (activador natural de los receptores de $\mathrm{GABA}_{A}$ y $\mathrm{GABA}_{B}$ ) como gaboxadol (agonista de $\mathrm{GABA}_{A}$ ) 
provocaban efectos similares a las neuronas, disminuyendo drásticamente su tasa de disparo, pero sin afectar en demasía el nivel de SSA. Como el efecto de las dos drogas era parejo, concluimos que la mayor parte del efecto que veíamos se mediaba por los receptores $\mathrm{GABA}_{\mathrm{A}}$. Por el contrario, el bloqueo de los receptores $\mathrm{GABA}_{\mathrm{A}}$ al usar la gabazina aumentaba la tasa de disparo a nivel general, tanto al estímulo estándar como al raro, haciendo disminuir el nivel de SSA.

\section{Conclusiones}

Junto a datos de estudios previos realizados en el IC (Pérez-González y col., 2012), estos experimentos apuntan a que la inhibición mediada por $\mathrm{GABA}_{\mathrm{A}}$ no genera la SSA, aunque el sistema GABAérgico parece estar ejerciendo un control de ganancia sobre dicho fenómeno. La presencia de un mismo fenómeno en dos núcleos distintos de la vía auditiva parece reflejar la existencia de sucesivos niveles de procesamiento jerárquico, sugiriendo que el sistema GABAérgico actúa a lo largo del sistema auditivo reduciendo la información redundante. 


\section{Resumen artículo III: SSA en animal despierto y anestesiado}

\section{Objetivos}

En el artículo anterior demostramos como el sistema GABAérgico ejercía un control de ganancia en la SSA. Curiosamente, la gran mayoría de anestesias usadas para la experimentación animal actúan sobre receptores GABAérgicos, alterando el equilibrio entre la excitación y la inhibición. Como la práctica totalidad de los experimentos se habían realizado bajo los efectos de la anestesia procedimos a realizar un análisis comparativo de los niveles de SSA entre animales despiertos y anestesiados.

\section{Metodología}

Implantamos en el laboratorio un sistema de registro de neuronas usado durante muchos años en murciélagos, la técnica de fijación de cabeza, adaptándolo para el registro de ratones despiertos (Bryant y col., 2009; Muniak y col., 2012). Por otro lado, los registros en animales anestesiados se hicieron con uretano, el anestésico cotidiano en el laboratorio y el usado en los dos estudios comentados anteriormente. Registramos 93 neuronas, 54 en animales despiertos y 39 en animales anestesiados, evaluando factores como la actividad espontánea o la tasa de disparo neuronal. Posteriormente, comparamos los niveles de SSA entre animales despiertos y anestesiados, comprobando factores como la tasa de repetición y el contraste entre las frecuencias usadas en el paradigma oddball.

\section{Resultados}

Nuestros datos demostraron que la anestesia no está afectando ni a las propiedades generales de respuesta -como la tasa de disparo o la latencia de las respuestas- ni a los niveles de SSA en el IC. No obstante, el grado general de inhibición se muestra muy afectado por la anestesia, reflejándose en los registros en animales despiertos con unos altos niveles de 
actividad espontánea (SR, por su nomenclatura anglosajona, spontaneous activity) y la aparición de unas neuronas 'inhibidas' que reducen (o paran completamente) su actividad espontánea al presentar un sonido. Además, los altos niveles de SR encontrados en el animal despierto parecen estar relacionados con unos niveles de SSA ligeramente más bajos.

\section{Conclusiones}

Este trabajo demuestra la validez de los estudios previos realizados en animales bajo los efectos de la anestesia, al demostrar niveles de SSA muy similar tanto en animal despierto como anestesiado. Además, el estudio también sugiere que la SSA pueda estar particularmente activa en estados similares al sueño -donde la actividad espontánea está muy reducida-, ya que el uretano parece mimetizar de manera fehaciente el estado de sueño profundo (Pagliardini y col., 2012, 2013). 


\section{Resumen artículo IV: Los canales de frecuencia determinan la SSA}

\section{Objetivos}

Considerando los datos obtenidos de los tres artículos anteriores y habiendo descartado como mecanismo generador de SSA el balance entre excitación e inhibición, retomamos la teoría acerca de la segregación de las aferentes para entender cuáles son las propiedades físicas del sonido que generan la SSA. Así pues, nos propusimos descubrir si existe SSA para sonidos que divergen en intensidad.

\section{Metodología}

En el último estudio, se registraron 132 neuronas en el IC de la rata para estimar qué sensibilidad presentan las neuronas a la SSA para sonidos que difieren tanto en frecuencia como en intensidad. Así pues, evaluamos la adaptación cruzada fijando un tono de bajas intensidades dentro del FRA (sonido prueba) y usando el paradigma oddball con un tono estándar repetitivo de mayor intensidad que el sonido prueba (sonido condicionante) y que va variando tanto en frecuencia como en intensidad. La evaluación de la adaptación cruzada con este método se realizó con el objetivo de establecer el área de frecuencias e intensidades dentro del FRA que condicionan la respuesta al sonido prueba de baja intensidad. Además, 30 neuronas fueron evaluadas con un nuevo paradigma de adaptación rápida, para corroborar los datos obtenidos con el paradigma oddball.

\section{Resultados}

Nuestros datos demuestran que el IC puede distinguir perfectamente sonidos que difieren en frecuencia, pero que no pueden discernir sonidos que únicamente difieren en intensidad, sugiriendo que la SSA puede estar estrechamente relacionada con mecanismos parecidos al enmascaramiento posterior (Nelson y col., 2009). Además, el análisis de la adaptación cruzada 
por medio del paradigma oddball y del de adaptación rápida permitió dibujar unos canales de frecuencia que se ensanchan al aumentar la intensidad y que son más estrechos en las altas frecuencias y en neuronas con altos niveles de SSA para frecuencia.

\section{Conclusiones}

Así pues, este último estudio muestra que la SSA puede ser evocada siempre y cuando el sonido condicionante de alta intensidad se encuentre fuera del canal de frecuencia que codifica para el sonido de baja intensidad. De esta manera, los resultados obtenidos en este estudio sugieren que la amplitud de los canales aferentes de frecuencia que llegan a la neurona son los que finalmente determinan la sensibilidad de la SSA para características físicas del sonido como la frecuencia y la intensidad. 


\section{Discusión}

En esta Tesis Doctoral se han realizado 4 estudios electrofisiológicos en la rata o el ratón en dos núcleos subcorticales: el IC y el MGB. Dichos estudios han revelado importantes características acerca de la SSA en el sistema auditivo de los mamíferos.

En el estudio I (Duque y col., 2012), se confirmó que las neuronas de las regiones no lemniscales del IC presentan altos niveles de SSA para frecuencia (Malmierca y col., 2009). Además, en el último estudio (Wang y col., 2014, en revisión) mostramos como las neuronas con estos altos niveles de SSA para frecuencia poseen unos canales de frecuencia más estrechos. Teniendo en cuenta que los árboles dendríticos de las neuronas de las regiones no lemniscales del IC son muy grandes y caóticos (Malmierca y col., 1993, 1995, 2011), podemos especular que esta desorganización dendrítica permite la integración de más (Duque y col., 2012) y más estrechos (Wang y col., 2014, en revisión) canales de frecuencia. Si así fuera, los altos niveles de SSA encontrados en las regiones corticales del IC estarían relacionados con el ancho de banda neuronal.

El primer estudio muestra también como las neuronas del IC tienen mayores niveles de SSA para sonidos de baja intensidad (Duque y col., 2012), mientras que el último demuestra que los canales de frecuencia se ensanchan a medida que se aumenta la intensidad del sonido (Wang y col., 2014, en revisión). Analizando conjuntamente dichos resultados, los bajos niveles de SSA a altas intensidades podrían ser fácilmente explicables debido a dicho ensanchamiento: a bajas intensidades el contraste de frecuencias no permite adaptación cruzada, pues las dos frecuencias estarían en dos canales de frecuencia diferentes. A altas intensidades, el canal sería más ancho que ese mismo contraste de frecuencia y la adaptación cruzada haría que los niveles de SSA a esa alta intensidad fueran prácticamente insignificantes. 
Además, las neuronas del IC son capaces de detectar sonidos de alta intensidad que se encuentran en un ambiente de bajas intensidades, pero no al revés (Wang y col., 2014, en revisión). Estos resultados sugieren que no existe sensibilidad de la SSA para sonidos que difieren en intensidad, ya que la codificación de la intensidad se encuentra dominada por fenómenos como el enmascaramiento posterior. Si así fuera, la falta de SSA para intensidad sería fácilmente explicable por el solapamiento de la actividad de las sinapsis para los sonidos de altas y bajas intensidades (Scholl y col., 2008), es decir, las sinapsis activadas por un sonido de alta intensidad estarían usando $-\mathrm{y}$ consiguientemente adaptando- las sinapsis activadas para un sonido de baja intensidad. Así pues, la codificación de la intensidad estaría dominada por la adaptación cruzada de altas a bajas intensidades y la auténtica discriminación entre intensidades (Jacobsen y col., 2003) sólo podría llevarse a cabo en regiones corticales superiores. Para desentrañar cuales son las características físicas del sonido que definen la SSA, también se trató de evocarla con sonidos raros que diferían tanto en frecuencia como en intensidad (Wang y col., 2014, en revisión). El análisis de estos datos permitió entender las bases de la adaptación cruzada: la SSA puede ser evocada siempre y cuando el sonido condicionante de alta intensidad se encuentre fuera del canal de frecuencia que codifica para el sonido de baja intensidad (Wang y col., 2014, en revisión). Estos datos encajan a la perfección con el modelo de SSA definido por depresión sináptica (Grill-Spector y col., 2006; Briley y Krumbholz, 2013). Dicho modelo está apoyado en la teoría de los canales de adaptación específicos para frecuencia (Eytan y col., 2003; Taaseh y col., 2011; Hershenhoren y col., 2014) y explica tanto los datos obtenidos en el estudio I para sonidos que defieren en frecuencia (Duque y col., 2012) como los que difieren en intensidad (Wang y col., 2014, en revisión).

Finalmente, el estudio I también demostró que los niveles de SSA para frecuencia eran mayores si los sonidos presentados estaban en el rango 
de altas frecuencias (Duque y col., 2012), posiblemente debido a que a altas frecuencias los canales de frecuencia son más estrechos (Wang y col., 2014, en revisión). Este fenómeno ha sido previamente observado tanto en las fibras del nervio auditivo (Westerman y Smith, 1985) como en el IC (Dean y col., 2008). El desarrollo de esta gran agudeza auditiva a altas frecuencias puede ser debido a la ingente cantidad de sonidos relevantes para la supervivencia de ratas y ratones en este espectro de sonidos.

Aunque parece claro que la SSA a niveles subcorticales está generada por mecanismos como la depresión sináptica, en el estudio II se usó la microiontoforesis para elucidar qué rol tiene el sistema inhibitorio GABAérgico en la generación o modulación de la SSA (Duque et al., 2014). La aplicación tanto de GABA como del agonista específico de $G_{A B} A_{A}$ gaboxadol aumentan la sensibilidad de la neurona a SSA al disminuir de forma generalizada la tasa de disparo neuronal. Por el contrario, el uso de la gabazina (antagonista específico de los receptores $\mathrm{GABA}_{A}$ ) aumenta la tasa de disparo neurona y disminuye la sensibilidad de la SSA. El efecto de GABA sobre la neurona es similar en todo el rango de frecuencias de cada neurona, lo que hace sospechar que la inhibición es un fenómeno independiente de las aferentes que generan la SSA. De hecho, en el estudio IV (Wang y col., 2014, en revisión) se muestra que la generación GABAérgica de la nomonotonicidad en el IC (Sivaramakrishnan y col., 2004; Grimsley y col., 2013) no permite mantener la sensibilidad a los sonidos de baja intensidad si el ambiente auditivo presenta sonidos de alta intensidad. Así pues, los datos apuntan a que la inhibición en el IC es un mecanismo que ocurre a posteriori de la generación de la SSA y que sirve para modular la sensibilidad neuronal al ejercer un control de ganancia (Robinson y McAlpine 2009; Isaacson y Scanziani 2011), tal y como se explica en el efecto iceberg (Rose y Blakemore 1974). 
Aun así, como la anestesia puede estar alterando el equilibrio entre la excitación y la inhibición, en el estudio III se implantó un método para registrar las neuronas del IC del ratón mientras el animal estuviera despierto (Duque y Malmierca, 2014). Dicho estudio muestra claramente como los niveles de SSA obtenidos en el procedimiento con el animal despierto son equiparables en magnitud a los niveles obtenidos en los procedimientos con animales anestesiados. Los datos también revelan que el animal despierto puede encontrarse en una situación pre-adaptativa (Abolafia y col., 2011), debido a que los niveles de actividad espontánea (SR, spontaneous rate) sí se muestran claramente alterados por la anestesia. Así pues, como los altos niveles de SR en animales despiertos están relacionados con un descenso de la adaptación (Chung y col., 2002; Castro-Alamancos, 2004), la SR podría estar modulando las respuestas neuronales (Buran y col., 2014). Como los niveles más altos de SSA se corresponden con los niveles más bajos de SR (Duque y Malmierca, 2014), los datos sugieren que la inhibición GABAérgica mostrada anteriormente (Pérez-González y col., 2012; Duque y col., 2014) podría estar modulando activamente los niveles de SR. Además, como estudios recientes muestran que el uretano produce una réplica casi exacta del estado de reposo que existe durante el sueño profundo (Clement y col., 2008; Pagliardini y col., 2012, 2013), en este trabajo especulamos que durante dicho estado se pueda requerir una respuesta acentuada a las señales de peligro y que, por lo tanto, la atenuación de la SR por el sistema GABAáergico esté acentuando la sensibilidad de la SSA mientras los animales están durmiendo (Duque y Malmierca, 2014).

En el futuro, sería interesante entender cuáles son los mecanismos sinápticos que llevan a la generación de la SSA. La depresión sináptica es un buen candidato, pues que se trata de un mecanismo específico de las aferentes neuronales y dependiente de las respuestas previas de dichas aferentes (Abbot y col., 1997; Rothman y col., 2009). No obstante, la 
depresión sináptica no tiene por qué ser un mecanismo pasivo, ya que actualmente se conocen multitud de vías de señalización retrógradas formadas por gases (óxido nítrico), péptidos (dinorfinas), factores de crecimiento (BDNF) e incluso aminoácidos convencionales (GABA, glutamato). En el cerebro de los mamíferos el sistema de los endocanabinoides (eCB) podría perfectamente ejercer dicha función (Castillo et al., 2012) pues regula la fuerza de las aferentes al secretarse a nivel sináptico retrógradamente. Curiosamente, el sistema de eCB ha demostrado mediar mecanismos como la depresión a corto término en núcleos cocleares (Zhao y col., 2009; Zhao y Tzounopoulos 2011), el complejo olivar superior (Trattner y col., 2013) e incluso en la corteza lateral del IC (Penzo and Peña, 2009).

En resumen, esta Tesis Doctoral demuestra que la SSA es un mecanismo presente en el cerebro del mamífero y que no se trata de un artefacto generado por la anestesia. Muestra además que la SSA es un mecanismo que puede explicarse perfectamente, a nivel subcortical, por el modelo de los canales de frecuencia. La existencia de controles de ganancia consecutivos ejercidos por el sistema GABAérgico sugiere también la presencia de varios niveles jerárquicos de procesamiento que ayudan a refinar y reducir la información redundante. En conjunto, la SSA parece ser un mecanismo que actúa como filtro preatentivo reduciendo las señales sensoriales irrelevantes, ayudando a los animales a presentar respuestas adecuadas para facilitar su supervivencia. 


\section{Conclusiones}

1. La SSA no es constante dentro del FRA y, por consiguiente, no es una propiedad característica de la neurona. La SSA es más fuerte a bajas intensidades y está sesgada hacia altas frecuencias.

2. Las neuronas localizadas en las regiones no lemniscales del IC presentan los niveles de SSA más altos. Dichas regiones se caracterizan por tener discretas respuestas neuronales tipo on y amplios FRAs que integran grandes rangos de frecuencia.

3. El sistema GABAérgico modula la SSA en el MGB ejerciendo un control de ganancia. Así pues, GABA hace disminuir la tasa de respuesta neuronal e incrementar el nivel de SSA, pero no lo está generando.

4. La modulación GABAérgica de la SSA se realiza básicamente a través de los receptores $G A B A_{A}$.

5. SSA es un fenómeno no atribuible a la anestesia y los niveles observados en animal despierto y anestesiado son comparables.

6. La SSA está dinámicamente modulada por el estado del animal a través del control de la actividad espontánea.

7. Las neuronas del IC no muestran SSA para sonidos que sólo difieren en intensidad, pero ésta puede obtenerse siempre y cuando el sonido condicionante de alta intensidad se encuentre fuera del canal de frecuencia que codifica para el sonido de baja intensidad.

8. Las neuronas con altos niveles de SSA para diferencias de frecuencia tienen canales de frecuencia más estrechos. Dichos canales se ensanchan al aumentar la intensidad del sonido y son más estrechos a altas frecuencias. 


\section{Referencias}

Abolafia, J.M., Vergara, R., Arnold, M.M., Reig, R. and Sanchez-Vives, M.V. (2011) Cortical auditory adaptation in the awake rat and the role of potassium currents. Cereb Cortex, 21, 977-990.

Abbott, L.F., Varela, J.A., Sen, K. and Nelson, S.B. (1997) Synaptic depression and cortical gain control. Science 275: 220-224.

Anderson, L.A., Christianson, G.B. and Linden, J.F. (2009) Stimulus-specific adaptation occurs in the auditory thalamus. J Neurosci, 29, 7359-7363.

Anderson, L.A. and Malmierca, M.S. (2013) The effect of auditory cortex deactivation on stimulus-specific adaptation in the inferior colliculus of the rat. Eur J Neurosci, 37, 52-62.

Antunes, F.M. and Malmierca, M.S. (2011) Effect of auditory cortex deactivation on stimulus-specific adaptation in the medial geniculate body. J Neurosci, 31, 17306-17316.

Antunes, F.M. and Malmierca, M.S. (2014) An Overview of Stimulus-Specific Adaptation in the Auditory Thalamus. Brain Topogr. 27(4):480-99.

Antunes, F.M., Nelken, I., Covey, E. and Malmierca, M.S. (2010) Stimulusspecific adaptation in the auditory thalamus of the anesthetized rat. PLoS One, 5, e14071.

Assisi, C., Stopfer, M., Laurent, G. and Bazhenov, M. (2007) Adaptive regulation of sparseness by feedforward inhibition. Nat Neurosci, 10, 1176-1184.

Attneave, F. (1954) Some informational aspects of visual perception. Psychological review, 61, 183-193. 
Ayala, Y.A. and Malmierca, M.S. (2013) Stimulus-specific adaptation and deviance detection in the inferior colliculus. Front Neural Circuits, 6, 89.

Ayala, Y.A., Perez-Gonzalez, D., Duque, D., Nelken, I. and Malmierca, M.S. (2013) Frequency discrimination and stimulus deviance in the inferior colliculus and cochlear nucleus. Front Neural Circuits, 6, 119.

Barlow, H. B. (1961) Possible principles underlying the transformation of sensory messages. In 'Sensory Communication' (ed. Rosenbluth, W. A.) 217-234 (MIT Press, Cambridge).

Bendixen, A., SanMiguel, I. and Schroger, E. (2012) Early electrophysiological indicators for predictive processing in audition: a review. Int $J$ Psychophysiol, 83, 120-131.

Bregman, A.S. (1990). Auditory Scene Analysis: The Perceptual Organization of Sound. Cambridge, Massachusetts: The MIT Press.

Briley, P.M. and Krumbholz, K. (2013) The specificity of stimulus-specific adaptation in human auditory cortex increases with repeated exposure to the adapting stimulus. J Neurophysiol, 110, 2679-2688.

Buran, B.N., von Trapp, G. and Sanes, D.H. (2014) Behaviorally gated reduction of spontaneous discharge can improve detection thresholds in auditory cortex. J Neurosci, 34, 4076-4081.

Calford, M.B. and Aitkin, L.M. (1983) Ascending projections to the medial geniculate body of the cat: evidence for multiple, parallel auditory pathways through thalamus. J Neurosci, 3, 2365-2380.

Castro-Alamancos, M.A. (2004) Absence of rapid sensory adaptation in neocortex during information processing states. Neuron, 41, 455-464. 
Clement, E.A., Richard, A., Thwaites, M., Ailon, J., Peters, S. and Dickson, C.T. (2008) Cyclic and sleep-like spontaneous alternations of brain state under urethane anaesthesia. PLoS One, 3, e2004.

Dahmen, J.C., Keating, P., Nodal, F.R., Schulz, A.L. and King, A.J. (2010) Adaptation to stimulus statistics in the perception and neural representation of auditory space. Neuron, 66, 937-948.

de Ribaupierre, F. (1997). Acoustical information processing in the auditory thalamus and cerebral cortex. In 'Anatomical and functional aspects of the cochlear nucleus' (Ehret, G., and Romand, R., Eds.). pp. 317-397. Oxford Univerity Press. Oxford.

Dean, I., Harper, N.S. and McAlpine, D. (2005) Neural population coding of sound level adapts to stimulus statistics. Nat Neurosci, 8, 1684-1689.

Doron, N.N. and Ledoux, J.E. (1999) Organization of projections to the lateral amygdala from auditory and visual areas of the thalamus in the rat. $J$ Comp Neurol, 412, 383-409.

Duque, D. and Malmierca, M.S. (2014) Stimulus-specific adaptation in the inferior colliculus of the mouse: anesthesia and spontaneous activity effects. Brain Struct Funct. Epub ahead of print.

Duque, D., Malmierca, M.S. and Caspary, D.M. (2014) Modulation of stimulus-specific adaptation by GABAA receptor activation or blockade in the medial geniculate body of the anaesthetized rat. J Physiol, 592, 729-743.

Duque, D., Perez-Gonzalez, D., Ayala, Y.A., Palmer, A.R. and Malmierca, M.S. (2012) Topographic distribution, frequency, and intensity dependence of stimulus-specific adaptation in the inferior colliculus of the rat. $J$ Neurosci, 32, 17762-17774. 
Escera, C. and Malmierca, M.S. (2013) The auditory novelty system: An attempt to integrate human and animal research. Psychophysiology, 51, 111-123.

Eytan, D., Brenner, N. and Marom, S. (2003) Selective adaptation in networks of cortical neurons. J Neurosci, 23, 9349-9356.

Fairhall, A.L., Lewen, G.D., Bialek, W. and de Ruyter Van Steveninck, R.R. (2001) Efficiency and ambiguity in an adaptive neural code. Nature, 412, 787-792.

Faye-Lund, H. and Osen, K.K. (1985) Anatomy of the inferior colliculus in rat. Anat Embryol (Berl), 171, 1-20.

Fishman, Y.I. (2013) The Mechanisms and Meaning of the Mismatch Negativity. Brain Topogr. 27(4):500-26.

Fishman, Y.I. and Steinschneider, M. (2010) Neural correlates of auditory scene analysis based on inharmonicity in monkey primary auditory cortex. J Neurosci, 30, 12480-12494.

Friston, K. (2005) A theory of cortical responses. Philos Trans $R$ Soc Lond B Biol Sci. Apr 29;360(1456):815-36.

Grill-Spector, K., Henson, R. and Martin, A. (2006) Repetition and the brain: neural models of stimulus-specific effects. Trends Cogn Sci, 10, 14-23.

Grimsley, C.A., Sanchez, J.T. and Sivaramakrishnan, S. (2013) Midbrain local circuits shape sound intensity codes. Front Neural Circuits 7:174.

Hershenhoren, I., Taaseh, N., Antunes, F.M. and Nelken, I. (2014) Intracellular correlates of stimulus-specific adaptation. J Neurosci, 34, 3303-3319.

Hu, B. (2003) Functional organization of lemniscal and nonlemniscal auditory thalamus. Exp Brain Res, 153, 543-549. 
Hu, B., Senatorov, V. and Mooney, D. (1994) Lemniscal and non-lemniscal synaptic transmission in rat auditory thalamus. J Physiol, 479 (Pt 2), 217-231.

Isaacson, J.S. and Scanziani, M. (2011) How inhibition shapes cortical activity. Neuron, 72, 231-243.

Jacobsen, T., Horenkamp, T. and Schröger, E. (2003) Preattentive memorybased comparison of sound intensity. Audiol Neurootol, 8, 338-346.

Katz, Y., Heiss, J.E. and Lampl, I. (2006) Cross-whisker adaptation of neurons in the rat barrel cortex. J Neurosci, 26, 13363-13372.

Klug, A. and Grothe, B. (2010) Ethological stimuli. In: Rees, A. and Palmer, A.R. (eds) Oxford Handbook of Auditory Science: The Auditory Brain. Oxford University Press, ISBN 978-0-19-923328-1, pp. 171-190

Komura, Y., Tamura, R., Uwano, T., Nishijo, H., Kaga, K. and Ono, T. (2001) Retrospective and prospective coding for predicted reward in the sensory thalamus. Nature 412:546-549.

Komura, Y., Tamura, R., Uwano, T., Nishijo, H. and Ono, T. (2005) Auditory thalamus integrates visual inputs into behavioral gains. Nat. Neurosci. 8: 1203-1209.

Kostal, L., Lansky, P. and Rospars, J.P. (2008) Efficient olfactory coding in the pheromone receptor neuron of a moth. PLoS Comput Biol, 4, e1000053.

Lee, C.C. and Sherman, S.M. (2010) Topography and physiology of ascending streams in the auditory tectothalamic pathway. Proc Natl Acad Sci U S A, 107, 372-377.

Lee, C.C. and Sherman, S.M. (2011) On the classification of pathways in the auditory midbrain, thalamus, and cortex. Hear Res, 276, 79-87. 
Lewicki, M.S. (2002) Efficient coding of natural sounds. Nat Neurosci, 5, 356363.

Loftus, W.C., Malmierca, M.S., Bishop, D.C. and Oliver, D.L. (2008) The cytoarchitecture of the inferior colliculus revisited: a common organization of the lateral cortex in rat and cat. Neuroscience, 154, 196-205.

Malmierca, M.S. (2003) The structure and physiology of the rat auditory system: an overview. Int Rev Neurobiol, 56, 147-211.

Malmierca, M. S., and Hackett, T. A. (2010). Structural organization of the ascending auditory pathway. In: Rees, A. and Palmer, A.R. (eds) The Oxford Handbook of Auditory Science: The Auditory Brain. Oxford University Press, ISBN 978-0-19-923328-1, pp. 9-41

Malmierca, M.S., Blackstad, T.W. and Osen, K.K. (2011) Computer-assisted 3D reconstructions of Golgi-impregnated neurons in the cortical regions of the inferior colliculus of rat. Hear Res, 274, 13-26.

Malmierca, M.S., Seip, K.L. and Osen, K.K. (1995) Morphological classification and identification of neurons in the inferior colliculus: a multivariate analysis. Anat Embryol (Berl), 191, 343-350.

Malmierca, M.S., Blackstad, T.W., Osen, K.K., Karagulle, T. and Molowny, R.L. (1993) The central nucleus of the inferior colliculus in rat: a Golgi and computer reconstruction study of neuronal and laminar structure. $J$ Comp Neurol, 333, 1-27.

Malmierca, M.S., Cristaudo, S., Perez-Gonzalez, D. and Covey, E. (2009) Stimulus-specific adaptation in the inferior colliculus of the anesthetized rat. J Neurosci, 29, 5483-5493. 
Maravall, M., Petersen, R.S., Fairhall, A.L., Arabzadeh, E. and Diamond, M.E. (2007) Shifts in coding properties and maintenance of information transmission during adaptation in barrel cortex. PLoS Biol, 5, e19.

May, P.J. and Tiitinen, H. (2010) Mismatch negativity (MMN), the devianceelicited auditory deflection, explained. Psychophysiology, 47, 66-122.

Näätänen, R., Gaillard, A.W. and Mantysalo, S. (1978) Early selectiveattention effect on evoked potential reinterpreted. Acta Psychol (Amst), 42, 313-329.

Näätänen, R., Pääviläinen, P., Tiitinen, H., Jiang, D. and Alho, K. (1993) Attention and mismatch negativity. Psychophysiology, 30, 436-450.

Näätänen, R., Tervaniemi, M., Sussman, E., Pääviläinen, P. and Winkler, I. (2001) "Primitive intelligence" in the auditory cortex. Trends Neurosci, 24, 283-288.

Nelken, I. (2014) Stimulus-specific adaptation and deviance detection in the auditory system: experiments and models. Biol Cybern. Epub ahead of print.

Pagliardini, S., Funk, G.D. and Dickson, C.T. (2013) Breathing and brain state: urethane anesthesia as a model for natural sleep. Respiratory physiology and neurobiology, 188, 324-332.

Pagliardini, S., Greer, J.J., Funk, G.D. and Dickson, C.T. (2012) Statedependent modulation of breathing in urethane-anesthetized rats. $J$ Neurosci, 32, 11259-11270.

Penzo, M.A. and Peña, J.L. (2009) Endocannabinoid-mediated long-term depression in the avian midbrain expressed presynaptically and postsynaptically. J Neurosci, 29:4131-4139. 
Pérez-González, D., Hernandez, O., Covey, E. and Malmierca, M.S. (2012) GABA(A)-Mediated Inhibition Modulates Stimulus-Specific Adaptation in the Inferior Colliculus. PLoS One, 7, e34297.

Pérez-González, D., Malmierca, M.S. and Covey, E. (2005) Novelty detector neurons in the mammalian auditory midbrain. Eur $J$ Neurosci, 22, 2879-2885.

Robinson, B.L. and McAlpine, D. (2009) Gain control mechanisms in the auditory pathway. Curr Opin Neurobiol, 19, 402-407.

Rose, D. and Blakemore, C. (1974) Effects of bicuculline on functions of inhibition in visual cortex. Nature, 249, 375-377.

Rothman, J.S., Cathala, L., Steuber, V. and Silver, R.A. (2009) Synaptic depression enables neuronal gain control. Nature 457: 1015-1018.

Rudolph, U. and Antkowiak, B. (2004) Molecular and neuronal substrates for general anaesthetics. Nat Rev Neurosci, 5, 709-720.

Saldaña, E., Feliciano, M. and Mugnaini, E. (1996) Distribution of descending projections from primary auditory neocortex to inferior colliculus mimics the topography of intracollicular projections. J Comp Neurol, 371, 15-40.

Scholl, B., Gao, X. and Wehr, M. (2008) Level dependence of contextual modulation in auditory cortex. J Neurophysiol, 99, 1616-1627.

Schröger, E. and Wolff, C. (1998) Attentional orienting and reorienting is indicated by human event-related brain potentials. Neuroreport, $\mathbf{9}$, 3355-3358.

Shannon, C.E. (1948) A mathematical theory of communication. Bell System Technical Journal 27, 379-423 and 623-656. 
Sherman, S.M. (2007) The thalamus is more than just a relay. Curr. Opin. Neurobiol. 17:417-422.

Sivaramakrishnan, S., Sterbing-D'Angelo, S.J., Filipovic, B., D'Angelo, W.R., Oliver, D.L. and Kuwada, S. (2004) GABA(A) synapses shape neuronal responses to sound intensity in the inferior colliculus. I Neurosci, 24:5031-5043.

Smith, P.H. and Spirou, G.A. (2002) From the cochlea to the cortex and back. In 'Integrative functions in the mammalian auditory pathway. Springer handbook of auditory research' (Oertel D, Fay, RR and Popper AN Eds.). pp. 6-71. Springer. New York.

Smirnakis, S.M., Berry, M.J., Warland, D.K., Bialek, W. and Meister, M. (1997) Adaptation of retinal processing to image contrast and spatial scale. Nature, 386, 69-73.

Taaseh, N., Yaron, A. and Nelken, I. (2011) Stimulus-specific adaptation and deviance detection in the rat auditory cortex. PLoS One, 6, e23369.

Tiitinen, H., May, P., Reinikainen, K. and Näätänen, R. (1994) Attentive novelty detection in humans is governed by pre-attentive sensory memory. Nature, 372, 90-92.

Trattner, B., Berner, S., Grothe, B. and Kunz, L. (2013) Depolarization-induced suppression of a glycinergic synapse in the superior olivary complex by endocannabinoids. J Neurochemistry 127:78-90.

Ulanovsky, N., Las, L., Farkas, D. and Nelken, I. (2004) Multiple time scales of adaptation in auditory cortex neurons. J Neurosci, 24, 10440-10453.

Ulanovsky, N., Las, L. and Nelken, I. (2003) Processing of low-probability sounds by cortical neurons. Nat Neurosci, 6, 391-398.

von Békésy G (1960) Experiments in hearing. New York: Mc Graw-Hill. 
Wark, B., Lundstrom, B.N. and Fairhall, A. (2007) Sensory adaptation. Curr Opin Neurobiol, 17, 423-429.

Westerman, L.A. and Smith, R.L. (1985) Rapid adaptation depends on the characteristic frequency of auditory nerve fibers. Hear res, 17:197-198.

Winer, J.A. (1985) The medial geniculate body of the cat. Advances in anatomy, embryology, and cell biology, 86, 1-97.

Winkler, I., Denham, S.L. and Nelken, I. (2009) Modeling the auditory scene: predictive regularity representations and perceptual objects. Trends Cogn Sci, 13, 532-540.

Yu, X.J., Xu, X.X., He, S. and He, J. (2009) Change detection by thalamic reticular neurons. Nat Neurosci, 12, 1165-1170.

Zhao, Y. and Tzounopoulos, T. (2011) Physiological activation of cholinergic inputs controls associative synaptic plasticity via modulation of endocannabinoid signaling. J Neurosci, 31:3158-3168.

Zhao, Y., Rubio, M.E. and Tzounopoulos, T. (2009) Distinct functional and anatomical architecture of the endocannabinoid system in the auditory brainstem. J Neurophysiol, 101:2434-2446. 